Simulating Bluff-body Flameholders: On the Use of Proper Orthogonal Decomposition for Combustion Dynamics Validation

\author{
Ryan Paul Blanchard
}

Dissertation submitted to the faculty of the Virginia Polytechnic Institute and State University in partial fulfillment of the requirements for the degree of

\author{
Doctor of Philosophy \\ In \\ Mechanical Engineering
}

\author{
Wing F. Ng \\ Uri Vandsburger \\ Srinath V. Ekkad \\ Danesh K. Tafti \\ Kevin T. Lowe \\ Lin Ma
}

April 16, 2014

Blacksburg, Virginia, USA

Keywords: Large Eddy Simulation, Combustion, Proper Orthogonal Decomposition, Validation 


\title{
Simulating Bluff-body Flameholders: On the Use of Proper Orthogonal Decomposition for Combustion Dynamics Validation
}

\author{
Ryan Paul Blanchard
}

\begin{abstract}
Contemporary tools for experimentation and computational modeling of unsteady reacting flow open new opportunities for engineering insight into dynamic phenomena. In the work presented here, a novel use of proper orthogonal decomposition (POD) is described to validate the structure of dominant heat release and flow features in the flame, shear-layer, and wake of a bluff-body-stabilized flame. A general validation process is presented which involves a comparison of experimental and computational results, beginning with single-point mean statistics and then extending to the dynamic modes of the data using POD to reduce the ensemble of instantaneous flow field snapshots. The results demonstrate the use of this technique by applying it to large eddy simulations of the bluff body stabilized premixed combustion experiment.
\end{abstract}

Large-eddy simulations (LES) using both Fluent and OpenFOAM were conducted to reproduce experiments conducted in an experimental test rig which was built as part of this work to study the behavior of turbulent premixed flames stabilized by bluff bodies. Planar ParticleImage Velocimetry (PIV) and filtered chemiluminescence were used to characterize the flow in the experiment's reacting and non-reacting regimes respectively. While PIV measurements could be compared directly to the velocity field in the simulations, the chemiluminescence measurements represented a line-of sight signal which was not directly comparable to the LES model. To account for this, the heat release in the LES models was integrated along simulated lines of sight by solving an additional discretized differential equation with heat release as the source term.

The results show generally good agreement between the dominant modes of the experiment with those of the numerical simulations. By isolating the dynamic modes from each other via the proper orthogonal decomposition, it was shown the models were able to accurately reproduce the size, shape, amplitude, and timescale of various dynamic modes which exist the experiment, some of which are dwarfed by the other flow features and are not apparent using time-averaging approaches or by inspection of instantaneous snapshots of the flow. 


\section{Acknowledgements}

I would like to thank Victoria, Katherine, and Elisabeth for the support they have given me, directly and indirectly while I have pursued this degree. Their selflessness and emotional support has been invaluable to me over the course of my $\mathrm{PhD}$ work and I am as grateful to them for it as I am sure that I did not deserve it.

Many thanks go to Dr. Wing Ng for the guidance, encouragement, tough questions, and sound advice given that smoothed the way for me to complete this degree. Without him bringing me into the project and keeping me on track, I shudder to think how my PhD work may have turned out. I also appreciate his vision in allowing me to pursue a dissertation topic that spanned the divide between experimental and computational research. I would also like to thank Dr. Todd Lowe for being an excellent technical resource to me to answer the many questions I have had. I feel I owe Dr. Srinath Ekkad a great deal for securing the funding that allowed me to do my $\mathrm{PhD}$ work without having to worry excessively about the finances of the project. To Dr. Lin Ma I say "thank you" for providing an example of academic excellence that has significantly affected my career and personal goals. I would also like to thank Dr. Uri Vandsburger for his stewardship of the laboratory where this work was done.

I owe an enormous debt of gratitude to my fellow graduate students who evened out the ups and downs of life as a graduate student and gave me plenty of opportunities to take a few steps back to see the bigger picture and remember that life is about more than combustion experiments. In particular, I'd like to thank Adam Janka for the excellent advice and feedback he has given me as an objective outsider to my project. To the students that worked directly with me on the project, AJ Wickersham, Cory Fleischman, Isaac Yeaton, and Craig English, I thank you all for the hard work, late nights, and early mornings to meet our deadlines and get our work done. To the students who came before me in the CSDL, Jordan Farina and Sam Shiver, thank you for holding my hand to help me acclimate to life at Virginia Tech, I have appreciated it very much.

I would like to thank Rolls Royce for the financial support that made this project possible. I would also like to specifically thank Duane Smith, Tim Roesler, and Keith McCormick for the expert advice in getting our experiments working properly. 
Table of Contents

Paper \#1 - Simulating Bluff-body Flameholders: On the Use of Proper Orthogonal Decomposition for Wake Dynamics Validation

1

1.1 Abstract 1

1.2 INTRODUCTION 1

1.3 MOTIVATION FOR DECOMPOSITION TECHNIQUES 2

1.4 REVIEW OF LITERATURE 3

1.4.1 Wake Dynamics of Bluff Bodies 3

1.4.2 Proper Orthogonal Decomposition 5

1.4.3 Frequency Spectra of POD Coefficients 6

$\begin{array}{lll}\text { 1.4.4 Extended POD } & 6\end{array}$

1.4.5 Non-reacting Large Eddy Simulation Method 7

1.5 EXPERIMENT 9

1.5.1 CSDL Facilities 9

$\begin{array}{ll}\text { 1.5.2 Rig Layout and Design } & 10\end{array}$

$\begin{array}{lll}1.5 .3 & \text { PIV } & 12\end{array}$

$\begin{array}{lll}\text { 1.6 LARGE EDDY SIMULATION } & 13\end{array}$

$\begin{array}{lll}\text { 1.6.1 Boundary Conditions } & 13\end{array}$

$\begin{array}{ll}\text { 1.6.2 Domain and Mesh } & 14\end{array}$

1.6.3 Comparison with Experiment 15

$\begin{array}{lll}1.7 & \text { RESULTS }\end{array}$

$\begin{array}{lll}\text { 1.7.1 Comparison of First Three Modes } & 18\end{array}$

1.7.2 Near-wall Sampling and Extended POD 21

$\begin{array}{lll}\text { 1.7.3 Boundary Layer } & 22\end{array}$

1.7.4 Periodic Sides 25

1.7.5 Turbulent Inlet 27

1.7.6 Refined Grid 29

$\begin{array}{lll}\text { 1.7.7 POD Convergence } & 32\end{array}$

$\begin{array}{lll}1.8 & \text { CONCLUSION } & 35\end{array}$

$\begin{array}{ll}\text { References } & 38\end{array}$ 
Paper \#2 - Simulating Bluff-body Flameholders: On the Use of Proper Orthogonal Decomposition for Combustion Dynamics Validation 43

$2.1 \quad$ ABSTRACT 43

2.2 INTRODUCTION 43

2.3 MOTIVATION FOR DECOMPOSITION TECHNIQUES 44

2.4 REVIEW OF LITERATURE 45

2.4.1 Wake Dynamics of Bluff Bodies 45

$2.5 \quad B L U F F$ BODY STABILIZED FLAMES 47

2.5.1 Proper Orthogonal Decomposition 52

2.6 EXPERIMENT

2.6.1 CSDL Facilities 53

2.6.2 Rig Layout and Design 54

$2.7 \quad$ LARGE EDDY SIMULATION 56

2.7.1 Comparison with Experiment 58

$\begin{array}{lll}2.8 & \text { RESULTS } & 60\end{array}$

2.8.1 Comparison of POD Modes 61

2.8.2 Wall and Midplane Heat Release vs Projection 63

2.8.3 Periodic Side Boundaries 64

2.8.4 Thicker Boundary Layer 65

2.9 Conclusions 66

References $\quad 70$

Paper \#3 - Study of Temperature Ratio Effects on Bluff Body Wake Dynamics Using Large Eddy Simulation and Proper Orthogonal Decomposition 77

$\begin{array}{lll}3.1 & \text { Abstract } & 77\end{array}$

$\begin{array}{lll}3.2 & \text { Introduction } & 77\end{array}$

$\begin{array}{lll}3.3 & \text { Review of Literature } & 78\end{array}$

3.3.1 Proper Orthogonal Decomposition $\quad 80$

3.4 Experiment 81

3.4.1 Particle Image Velocimetry $\quad 82$

3.4.2 Chemiluminescence 83

3.5 Premixed Combustion Simulations 83 
3.5.1 Domain, Mesh, and Boundary Conditions 86

3.5.2 Simulation of Line-of-Sight Chemiluminescence Measurement 88

$\begin{array}{lll}3.6 & \text { Results } & 90\end{array}$

3.6.1 Means Velocity Evolution with Temperature Ratio 92

3.6.2 POD Results 95

3.6.3 First Modes of Velocity and Chemiluminescence 96

$\begin{array}{lll}3.7 & \text { Conclusion } & 102\end{array}$

References 106

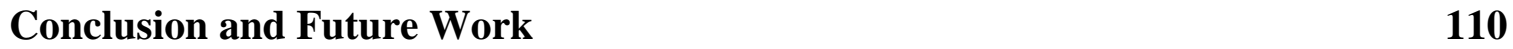

$\begin{array}{lll}\text { A } & \text { APPENDIX: Rig Drawings } & 113\end{array}$

B APPENDIX: Fluent UDF Codes 131

C APPENDIX: Data Reduction Codes 132

$\begin{array}{lll}\text { C.1 Vector POD Code } & 132\end{array}$

$\begin{array}{lll}\text { C.2 } & \text { FFT Code } & 134\end{array}$

C.3 Mode Aligning Code 135

C.4 Scalar Mode Plotter With Vee Gutter 135 


\section{List of Figures}

Figure 1.1 - Large eddy simulation of non-reacting flow around a bluff body from the current work showing iso-surfaces of the Q-criterion. The alternating shedding pattern in the bluff-body's wake can be seen.

Figure 1.2 - Test section temperature and Mach number limitations from facility constraints.

Figure 1.3 - Schematic of experiment showing the three sections of the rig and the locations of the fuel injection, vee-gutter flameholder, and windows. 10

Figure 1.4 - Transparent view of CAD model of the test section showing the locations of the three windows relative to the vee-gutter flameholder (and the PIV laser sheet).

Figure 1.5 - Isometric view of dimensions of the domain used in the LES models based on the width of the flameholder $\mathrm{w}=3.05 \mathrm{~cm}$. 14

Figure 1.6 - Plan view dimensions of the domain used in LES models, based on the dimension of the vee-gutter flameholder $\mathrm{w}=3.05 \mathrm{~cm}$ which has an included angle of $70^{\circ}$, 15

Figure 1.7 - Illustration of relative sizes and locations of sampling planes relative to the flameholder: 100x100 CFD sample grid points (blue) compared to the effective viewing area for the PIV measurements.

Figure 1.8 - Mean streamwise velocity plots as measured by Pitot probe and as simulated by Fluent LES.

Figure $1.9-v^{2}$ Reynolds stress spectra spectrum from LES model. Note the peak due to vortex shedding at $S t r=0.24$ and also the decay rate that seems to correspond to the $-5 / 3$ power law rule.

Figure 1.10 - Contours of the sum of the first three POD modes (left: v', right: u') obtained using results from PIV (top), Fluent (middle), and OpenFOAM (bottom). The profiles shown in figure 1.11 are taken along the white lines show on each plot. 18

Figure 1.11 - Comparison between numerical and experimental values of the first three POD modes. Left: v', right: u'. 19

Figure 1.12 - Contours of $\mathrm{u}$-velocity component of the sum of the first 3 POD modes of PIV (top), Fluent (middle), and OpenFOAM (bottom). The profiles shown in figure 13 are taken along the white lines shown imposed on each figure. 20 
Figure 1.13 - Plot of u-velocity component of the sum of the first 3 POD modes taken along the lines indicated in figure 1.12 .

Figure 1.14 - Plot of u-velocity component of the sum of the first 3 POD as isolated at $\mathrm{y} / \mathrm{w}=0$ at the midplane, near the wall, and near the wall but with EPOD to recover the nearwall modeshapes using the eigenvectors of the midplane analysis. 22

Figure 1.15 - Comparison between the baseline domain (left) and the domain with the extended domain (right) with increased distance between the inlet and the vee-gutter to produce a bigger/thicker boundary layer in the vicinity of the vee-gutter. 23

Figure 1.16 - Comparison between the sum of the first three POD modes of the baseline simulation (top) and thicker boundary layer configuration (bottom). Contours of the Ucomponent (streamwise) of velocity are shown on the left and V-component contours are shown on the right 24

Figure 1.17 - Comparison of the v-components (vertical) of velocity of the sum of the first three modes of the baseline simulation and the configuration with the thicker boundary layer. 25

Figure 1.18 - Comparison between the sum of the first three POD modes of the baseline simulation (top) and periodic side "wall" configuration (bottom). Contours of the U-component (streamwise) of velocity are shown on the left and V-component contours are shown on the right.

Figure 1.19 - Comparison of the first v-components (vertical) of velocity of the sum of the first three modes between baseline simulation and the configuration with periodic sides.... 27

Figure 1.20 - Comparison between the sum of the first three POD modes of the baseline simulation (top) and turbulent inlet configuration (bottom). Contours of the U-component (streamwise) of velocity are shown on the left and V-component contours are shown on the right.

Figure 1.21 - Comparison of the v-components (vertical) of velocity of the sum of the first three modes of the baseline simulation and the configuration with the turbulent inlet........ 29

Figure 1.22 - Velocity contours of first three modes of baseline (top) and refined grid (bottom) simulations, showing contours of u' (left) and v' (right)..... 30

Figure 1.23 - Comparison of the v-components (vertical) of velocity of the sum of the first three modes of the baseline simulation and the refined grid simulation. 31 
Figure 1.24 - Pope Criterion contours at midplane for baseline (top) and refined mesh (bottom) cases. .

Figure 1.25 - Computation time vs number of snapshots $\mathrm{N}$ showing that the computational time grows proportional to $\mathrm{N}^{3}$ as expected (green line is $\sim \boldsymbol{N 3}$ )...... 33

Figure 1.26 - Second order convergence rate of the first five eigenvalues..................... 34

Figure 1.27 - Convergence of First Three POD Modes with Increasing Number of Samples showing a first order convergence rate. 34

Figure 2.1 - Large eddy simulation from current work of non-reacting flow around a bluff body. The alternating shedding pattern in the bluff-body's wake can be seen from pattern of the vorticity isosurfaces. 45

Figure 2.2 - Schematic of a bluff-body stabilized premixed flame showing the recirculation of combustion products from the bluff-body's wake into the shear layer where it mixes and ignites the unburned fuel and air mixture.

Figure 2.3 - Envelope of section inlet temperature and Mach number limitations from facility constraints. 54

Figure 2.4 - Schematic of experiment showing the three sections of the rig and the locations of the fuel injection, vee-gutter flameholder, and windows. 55

Figure 2.5 - Transparent view of CAD model of the test section showing the two side windows and top window relative to the location of the vee-gutter flameholder..... 56

Figure 2.6 - Isometric view of dimensions of the domain used in the LES models based on the width of the flameholder $\mathrm{w}=3.05 \mathrm{~cm}$ and an included angle of $70^{\circ}$. 57

Figure 2.7 - Plan view dimensions of the domain used in LES models........................ 58

Figure 2.8 - Illustration of flame's heat release imaged to simulate the line-of-sight integrated chemiluminescence imaged in the experiment.

Figure 2.9 - Illustration of relative sizes and locations of sampling planes relative to the flameholder: 100x100 CFD sample grid points (blue) compared to the effective viewing area for the chemiluminescence measurements.

Figure 2.10 - Line of sight mean heat release fields as measured by chemiluminescence (left) and LES (right).

Figure 2.11 - Line of sight RMS heat release fields as measured by chemiluminescence (left) and LES (right). 61 
Figure 2.12 - Contours of the dominant POD modes of chemiluminescence (left) and the projection integral of heat release in the LES model (right). The Strouhal numbers of these modes were 0.24 and 0.29 respectively.

Figure 2.13 - Comparison between mode shapes of the second-most energetic pair of modes showing chemiluminescence data on the left and the baseline LES model on the right. The Strouhal numbers of these modes matched closely between experiment and simulation giving Str $=0.64$ and $S t r=0.63$ respectively. 62

Figure 2.14 - Contours of a low-frequency, low-energy mode revealed during POD of the chemiluminescence (left) and LES data (right). The spectra of these modes' POD coefficients showed peaks at Str $=0.037$ and $\operatorname{Str}=0.036$ respectively.

Figure 2.15 - Comparison betwen the dominant modes of the baseline LES model as determined by the line-of-sight-integrated heat release across the full width of the test section (left) and the local heat release at the midplane (middle) and near-wall (right) sampling locations.

Figure 2.16 - Dominant POD modes of the baseline simulation (left) and simulation with periodic sidewalls (right).

Figure 2.17 - Illustration of the geometry modfication to understand the effect of the boundary layer on the flame's dynamics. The baseline geometry is shown on the left and the extended geometry is shown on the right.

Figure 2.18 - Comparison between dominant POD modes of baseline simulation (left) and simulation with thicker boundary layer (right).

Figure 3.1 - Transparent view of CAD model of the test section showing the locations of the three windows relative to the vee-gutter flameholder. Flow direction is left-to-right. 82

Figure 3.2 - Transparent view of CAD model of the test section showing the locations of the three windows relative to the vee-gutter flameholder and the PIV laser sheet. Flow direction is left-to-right.

Figure 3.3 - Isometric view of dimensions of the domain used in the LES models based on the width of the flameholder $\mathrm{w}=3.05 \mathrm{~cm}$ and an included angle of $70^{\circ}$. 87

Figure 3.4 - Plan view dimensions of the domain used in LES models. 87

Figure 3.5 - Illustration of flame's heat release imaged to simulate the line-of-sight integrated chemiluminescence imaged in the experiment. 89 
Figure 3.6 - X-and y-locations of sampling points used for interrogating the velocity and heat release quantities in the flow. Also shown are the locations of the vee-gutter flamholder and the side window relative to the sampling grid and coordinate axes.

Figure 3.7 - Plot of time-averaged streamwise velocity profiles of the non-reacting condition at two locations downstream of the vee-gutter, $\mathrm{x} / \mathrm{w}=2.2$ (left) and $\mathrm{x} / \mathrm{w}=3.8$ (right)... 91

Figure 3.8 - Time-averaged chemiluminescence contours of the measured (left) and simulated (right) flames. Contours have arbitrary units. 91

Figure 3.9 - RMS contours of chemiluminescence contours of the measured (left) and simulated (right) flames. Contours have arbitrary units.

Figure 3.10 - Time-averaged u-velocity contours for the five conditions studied showing a clear pattern of wake-elongation with increasing heat release. 93

Figure 3.11 - Time-averaged line of sight reaction rate contours showing how the flame sheets' coalescence point moves farther downstream with increasing heat release until the two sheets finally remain separate permanently for the extreme case corresponding to the stoichiometric equivalence ratio. 94

Figure 3.12 - Comparison of the first POD modes of u-velocity (left) and v-velocity (right) between the PIV measurements (top) and large eddy simulation (bottom). 95

Figure 3.13 - Comparison between the first POD mode of line-of-sight chemiluminescence as measured in the experiment (top) and as simulated numerically (bottom). 96

Figure 3.14 - U-velocity contours of the first POD mode normalized by the bulk velocity. 98

Figure 3.15 - V-velocity contours of the first POD mode normalized by the bulk velocity. 99

Figure 3.16 - Line of sight reaction rate integral contours of the first POD mode....... 101

Figure 3.17 - Strouhal number of dominant shedding mode as a function of temperature ratio. 102 


\section{List of Tables}

Table 1.1 Boundary conditions for the simulations reported................................... 13

Table 2.1 - Boundary conditions for the simulations reported ...................................... 56

Table 3.1 - Temperature ratio parameter for each of the five simulations ...................... 88 


\section{Preface}

This dissertation has been compiled in manuscript format. It consists of three papers that together represent the progress made over the course of the author's $\mathrm{PhD}$ work and each has been submitted for publication as a separate journal article. The author is the lead author on all three papers and was directly involved with all aspects of the research including designing, building, and running the combustion rig used in the experimental side of the research as well as developing the numerical models that are validated against the experimental data. The first two papers have been accepted for publication by the ASME Journal of Engineering for Gas Turbines and Power (articles numbers GTP-14-1119 and GTP-14-1154) and the third paper has been submitted for publication to the journal Combustion and Flame. For brevity, the journal articles omit some information which is relevant to the work presented; this information is provided in the appendices.

The work reported here, both experimental as well as computational, was done in the combustion system dynamics laboratory (CSDL) at Virginia Tech. The lab has a long history of combustion-related research including combustion dynamics, multi-phase flows, development of diagnostic measurement techniques, and combustion emissions mitigation.

In combustors of all kinds, the combustion reactions which convert chemical energy into sensible heat also produce dilation in the working fluid. This dilation and its associated acceleration, create pressure fluctuations which, particularly in the case of premixed flames, can couple with the acoustics of the combustion chamber and other components of the combustion system. This acoustic coupling creates a feedback loop with the combustion reactions that can lead to thermo-acoustic instabilities which can contribute to decreased combustion efficiencies, increased levels of pollutant formation, and in extreme even combustor damage. Understanding how these thermoacoustic instabilities behave has been an active area of research for many years [1].

For bluff-body flameholders like the vee-gutter-type flameholder featured in this work, the flame is stabilized by recirculating hot combustion products from the vee-gutter's wake. This makes characterization of the vee-gutter's wake very important for understanding not just the flameholder's performance in both wet (fueled) and dry (unfueled) modes. Further, accurately modeling the dynamic behavior of the vee-gutters wake across the fueled/unfueled spectrum is 
critical since many of an afterburner's performance characteristics like ignition, turndown, lean blowout limit, and dry loss feature either substantially reduced reaction rates or no reactions at all. Numerical simulations of bluff-body flameholders, in either wet or dry operation, are often validated against experimental measurements using time-averages and point-wise statistics to evaluate the simulations predictive capabilities; however these techniques fail to capture the combined spatial and temporal nature of the dynamics of these systems, even for time-accurate simulation techniques like large eddy simulation, that have the potential to capture the combustion dynamics of interest [2-6].

To overcome the limitations of these validation methods, the papers comprising this dissertation propose and demonstrate the use of proper orthogonal decomposition (POD) to quantitatively compare and validate the shapes and amplitudes of the dynamic modes of LES models against experimental data and other simulations. This approach, including the treatment of line of sight chemiluminescence signals and described in detail in the papers and in the appendix, is, to the author's knowledge, novel.

Taken together, the three papers successively build on a common methodology of validating the dynamics of numerical simulations against experimental measurements but with each paper adding a substantial piece of physics that is being resolved or investigated. The first paper deals only with cold flow and the flow simulated flow field can be compared directly to the PIV measurements of the velocity field in the experiment. The second paper however deals with reacting flow which is validated against heat release measurements made via high-speed chemiluminescence which is a line-of-sight integral measurement for which significant changes to the numerical model, aside from the addition of the combustion reactions themselves, had to be added to the model in the first paper. Finally, the third paper builds on the first two by attempting to span the intermediate space between non-reacting and reacting cases of the same flow. To do this the velocity field as well as the line of sight techniques were employed however with the caveat that the model would integrate reaction rate along the sight lines rather than heat release.

The first paper deals with the use of (POD) for validation of the structure of dominant flow features in the wake of the bluff-body flameholder in the unfueled, non-reacting case. Large-eddy simulations (LES) using both Fluent and OpenFOAM were conducted based on experiments of the same geometry and at identical conditions. Particle-Image Velocimetry (PIV) 
measurements were taken at the center plane of the experimental test section. The validation process begins by comparing the time-average streamwise velocity profiles of the PIV and LES data and show good agreement. The dominant dynamic modes of the velocity field, as isolated using POD, were then compared against the same modes isolated from the LES data.

The results of these comparisons showed good agreement between the first modes of the velocity fields as measured by PIV and as simulated using LES in Fluent. The agreement was not as good for the same POD mode of the OpenFOAM LES data, which overpredicted the magnitude of the first mode's oscillations compared to the PIV measurement and Fluent simulation.

The sensitivity of this cold flow LES model was then examined by varying several parameters including mesh resolution, boundary conditions, and inflow turbulence while repeating the POD validation procedures. The results indicated an unexpected decoupling between the up- and downstream amplitudes of oscillations of the wake's shedding mode, indicating that the downstream oscillations are not simply artifacts of the upstream oscillations. This decoupling helps explain why the simulations on the coarser grid, which was somewhat under-resolved near the vee-gutter, could still match the PIV data measured farther downstream.

The second paper builds on the first by extending the technique to validate LES models of the reacting flow case. For this case, the combusting flow is measured in the experimental via high-speed imaging of the filtered chemiluminescent emissions of the turbulent, premixed flame, which has been shown to be well correlated with heat release [7 - 8]. The LES model of this case simulates the flame's heat release using a flame speed model which estimates the local reaction rate as a function of turbulence quantities and mesh resolution. Where in the first paper the velocity field at the midplane of the test section could be measured directly by PIV and sampled directly from the LES models, the chemiluminescence images represented a line-of sight measurement rather than heat release at a point. To account for this, the heat release in the LES models was integrated along simulated lines of sight by solving an additional discretized differential equation with heat release as the source term.

The results show good overall agreement between the shapes, magnitudes, and timescales of the primary modes of heat release of both the experiment and the simulation. In a particular, the first mode of heat release showed the same symmetric shedding pattern recovered from the chemiluminescence measurements and very similar length scales of these symmetric structures 
albeit with the LES flame showing a thinner flame brush and a somewhat higher frequency compared to the experiment. Additionally, good agreement was found between two additional modes of the simulated and experimental flames. First, a higher mode, by frequency very close to a harmonic of the first mode, was found to have been very closely matched in both space and frequency by the simulation and second, an unexpectedly good match was found for a lowenergy, low-frequency dynamic that exists in both the model and experiment as well.

A numerical study of into the effects of various simulation parameters on these heat release modes showed significant effects on the flame's effective angle but also on the size, shape, and symmetry patterns of the flame's dynamic modes. A significant change in the wake and flame structure was found when the side walls of the test section were changed from no-slip wall-type boundary conditions to periodic boundaries that could support mass- and momentumflux as well as non-zero gradients through these boundaries. With these new boundary types, the first mode of the flame's heat release transitioned from a fully-symmetric pattern with a high and a low on each side of the horizontal symmetry plane to asymmetric with a high on one side of the $\mathrm{x}$-axis and a corresponding low on the opposite side. This transition occurred with essentially zero change in this mode's frequency or energy and would have been effectively undetectable by simply examining the time averaged flame angles and frequency content of the pointwise heat release.

This result has huge implications not only for predicting which types of acoustic waves can or will couple with these heat release modes to cause combustion instabilities, but clearly illustrates one of the fundamental problems that has made combustion dynamics research so challenging, namely that the confined and unconfined versions of the same flameholder geometry can exhibit very different susceptibilities to thermoacoustic feedback without changing the frequency content of its preferred dynamic modes. This result sheds further light on other results in the literature that showed a link between confinement geometry, heat release, and evolving patterns of symmetry and anti-symmetry

However the real contribution from this paper is that by first validating the dynamics modes of the simulated confined flame against a similarly confined experimental flame, the changes in flame structure associated with the switch to the unconfined flame can be trusted as having come from a "validated model" and not be simply dismissed as a numerical artifact arising from the complexities turbulent premixed combustion modeling. 
In the third paper proper orthogonal decomposition (POD) is used to investigate how the dominant structures of a turbulent premixed flame change as a function of the amount of heat released in a bluff-body-stabilized turbulent premixed flame. The investigation uses large eddy simulations (LES) of the flame, the dynamics of which have been validated against experiments using particle image velocimetry (PIV), high-speed chemiluminescence imaging. The numerical simulations allow the effect of heat release to be decoupled from the laminar flame speed. Five simulations are reported here that vary the amount of heat released in the combustion reaction between 0 and $100 \%$ of the stoichiometric value, quantified by the temperature ratio of the burned to unburned gases, while holding constant both the laminar flame speed as well as the method of turbulent flame speed closure. The results indicate similar trends reported qualitatively by others, but by using POD to isolate the dominant heat release modes of each simulation, the decomposed data can clearly show how the previously-reported flow structures transition from asymmetric shedding in the case of zero heat-release to a fully symmetric shedding mode in the case of full heat release, which corroborate POD analyses of experimental investigations of similar phenomena [9-10].

Together, the three papers that make up this dissertation demonstrate a novel, efficient, and practical means of using POD for validating LES models against experimental data. Once validated, these models can then be used to investigate how the dynamics of the systems are affected by changes in either the physical processes that are part of the experiment or in the models that are used to describe them. The results of these investigations illustrate how significantly wake and flame structures can change as a result in any number of experimental or model parameters while providing a clear framework for future investigations of similar processes.

[1] Lieuwen, T. (2003) "Modeling Premixed Combustion-Acoustic Wave Interactions: A Review." Journal of Propulsion and Power, 19 (5) pp 765-781. doi:10.2514/2.6193.

[2] Macquisten, M., and Dowling, A., (1993) "Low Frequency Combustion Oscillations in a Model Afterburner." Combustion and Flame, 94 253-264. 
[3] Giacomazzi, E., Battaglia, V., and Bruno, C. (2004). "The coupling of turbulence and chemistry in a premixed bluff-body flame as studied by LES." Combustion and Flame, 138 (4), pp 320-335. doi:10.1016/j.combustflame.2004.06.004

[4] Erickson, R., Soteriou, M., and Mehta, P., (2006) “The Influence of Temperature Ratio on the Dynamics of Bluff Body Stabilized Flames." 44th AIAA Aerospace Sciences Meeting and Exhibit 2006 pp. 1-18.

[5] Shanbhogue, S., Husain, S., and Lieuwen, T. (2009). "Lean blowoff of bluff body stabilized flames: Scaling and dynamics." Progress in Energy and Combustion Science, 35

(1) pp 98-120. doi:10.1016/j.pecs.2008.07.003

[6] Cocks, P., Sankaran, V., and Soteriou, M., (2013) "Is LES of Reacting Flows Predictive? Part 1 : Impact of Numerics" $51^{\text {st }}$ AIAA Aerospace Sciences Meeting 2013, pp. 129.

[7] Haber, L.C., (2000) "An Investigation into the Origin, Measurement and Application of Chemiluminescent Light Emissions from Premixed Flames” M.S. thesis, Mechanical Engineering, Virginia Tech.

[8] Ranalli, J., (2009) "Spatially Resolved Analysis of Flame Dynamics for the Prediction of Thermoacoustic Combustion Instabilities.”, Ph.D. thesis, Mechanical Engineering Virginia Tech.

[9] Kostka, S., Roy, S., Huelskamp, B., Lynch, A., Kiel, B., and Gord, J., (2011) “Characterization of Bluff-Body-Flame Vortex Shedding Using Proper Orthogonal Decomposition" 49th AIAA Aerospace Sciences Meeting and Exhibit" pp. 1-7. 
[10] Kostka, S., Roy, S., Huelskamp, B., Lynch, A., Kiel, B., and Gord, J., (2012)

"Characterization of Flame-Shedding Behavior behind a Bluff-Body Using Proper

Orthogonal Decomposition.” Combustion and Flame, 159 (9) pp 2872-2882.

doi:10.1016/j.combustflame.2012.03.021. 


\section{Paper \#1 - Simulating Bluff-body Flameholders: On the Use of Proper Orthogonal Decomposition for Wake Dynamics Validation}

\subsection{Abstract}

In this article, we describe a novel use of proper orthogonal decomposition (POD) for validation of the structure of dominant flow features in the wake of a bluff-body flameholder. Large-eddy simulations (LES) using both Fluent and OpenFOAM were conducted based on experiments with planar Particle-Image Velocimetry (PIV) measurements of the same geometry and conditions. With the vision of extending the LES to reacting flows, a validation process is presented which involves a comparison of experimental and computational results, beginning with single-point mean statistics and then extended to the dynamic modes of the data sets as obtained using POD of the instantaneous flow field results. The results exhibit quantitative agreement between both shapes and mode magnitudes for the first POD modes of the measured and simulated data.

\subsection{INTRODUCTION}

The unsteady release of sensible heat and flow dilation in combustion processes create pressure fluctuations which, particularly in premixed flames, can couple with the acoustics of the combustion system. This acoustic coupling creates a feedback loop with the heat release that can lead to thermo-acoustic instabilities which can reduce combustion efficiency, increase pollutant formation, or become so severe that they damage the combustor. For vee-gutter-type bluff-body flameholders, the flame is stabilized by recirculating hot combustion products from the veegutter's wake. This makes understanding the flow characteristics of the vee-gutter wake very important for understanding not just the flameholder's performance in the wet (fueled) mode but in the dry (unfueled) mode as well since many of an afterburner's performance characteristics like ignition, turndown, lean blowout limit, and dry loss feature either substantially reduced reaction rates or no reactions at all.

Numerical simulations of bluff-body flameholders, in either wet or dry operation, are often validated against experimental measurements using time-averages and point-wise statistics to evaluate the predictive capabilities of simulations; however these techniques fail to capture the combined spatial and temporal nature of the dynamics of these systems. This work uses proper orthogonal decomposition (POD) to analyze both experimental and simulated data to isolate and then quantitatively compare the shapes and amplitudes of the dynamic modes of each against one another. 


\subsection{MOTIVATION FOR DECOMPOSITION TECHNIQUES}

Significant LES validation efforts against combustion experiments have been reported in the literature; these consist largely of comparisons between measured and simulated timeaverages, Reynolds decompositions, and power spectral density as validation metrics [1-4]. While successive moments of the time statistics do represent a natural and intuitively meaningful technique for the validation of most quantities for engineering purposes and although power spectral density does allow for detailed analysis of the frequency content of a given time series,

neither technique can provide an adequate description of the spatio-temporal nature of the system's dynamics. One approach which has been used to attempt to solve this problem is phaselocked sampling, which has been successfully used to study flames with periodic instabilities [5]. This technique however requires an external forcing source which can be used to trigger the measurement, preventing it from being applied to self-excited flames. Often in the open literature, qualitative 'validation' is presented for the spatial arrangement of a dominant flow phenomenon-instantaneous snapshots are shown. Such snapshots are clearly valuable for comparison, but due to the stochastic nature of unsteady turbulent flow, direct quantitative comparison of two flow realizations cannot be accomplished. Further, such qualitative comparison can obscure less-dominant flow features which may be of physical importance but are overshadowed by the propensity of the eye to interpret only the more dominant structures.

Decomposition techniques, such as proper orthogonal decomposition (POD) and dynamic mode decomposition (DMD) allow many of these shortcomings to be overcome and allow for reproducible, statistically converged representations of the key flow features and their various magnitudes, time scales, and spatial distributions. These techniques use instantaneous realizations of the system with sufficient spatial and temporal range and resolution to capture the relevant flow structures. Ensembles of these instantaneous snapshots can be decomposed in space and/or time to separate the various coherent modes from each other in a way that allows for a statistically representative, reproducible, and quantitative description of each. Because such decompositions rely only on the input data, requiring no underlying model or approximation, they are said to be agnostic to the source of the data; that is, the decomposition technique makes no distinction between data generated by a physical measurement or a numerical simulation. This feature makes these techniques well suited to the comparisons of measured and simulated dynamics shown here. 


\subsection{REVIEW OF LITERATURE}

This section provides a brief overview of the relevant literature for each of the key topics relevant to this work including: decomposition techniques including proper orthogonal decomposition and dynamic mode decomposition, premixed combustion, bluff-body stabilized premixed flames, and large-eddy simulation of premixed flames.

\subsubsection{Wake Dynamics of Bluff Bodies}

The wakes of bluff bodies immersed in non-reacting flows is a topic that has been thoroughly studied through both experimental [6-7] and numerical means [8-10]. Such wakes typically exhibit an alternating shedding pattern where the bluff body sheds vortices from alternating sides as shown in figure 1.1.

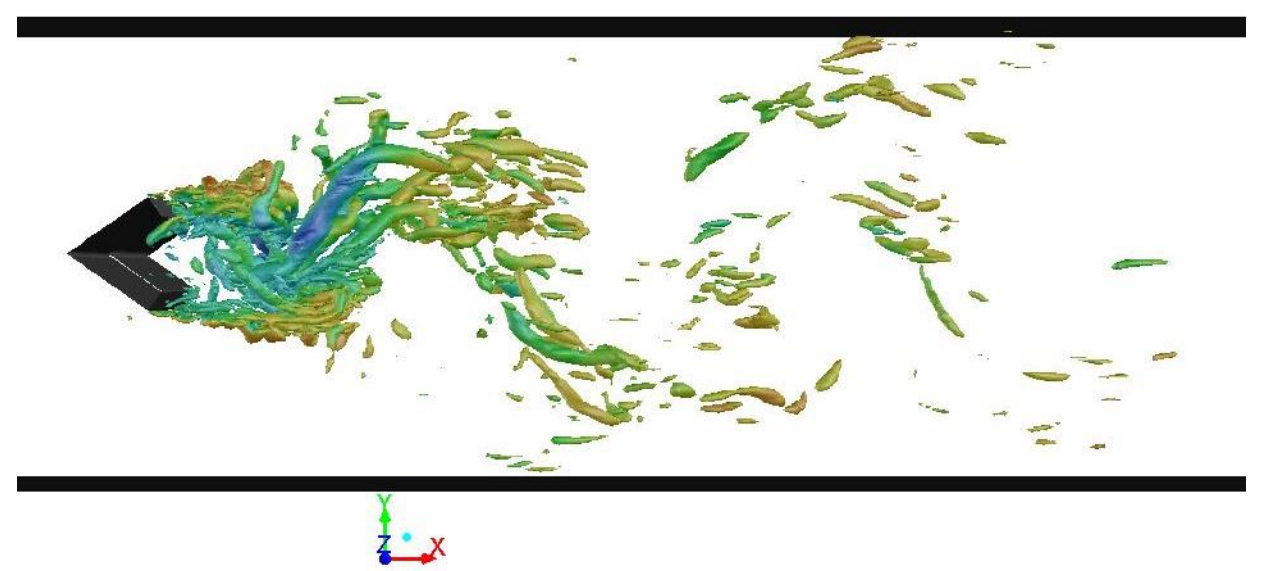

Figure 1.1 - Large eddy simulation of non-reacting flow around a bluff body from the current work showing iso-surfaces of the Q-criterion. The alternating shedding pattern in the bluffbody's wake can be seen.

The frequency of this shedding, typically non-dimensionalized as the Strouhal number

$$
\operatorname{Str}=\frac{\mathrm{fw}}{\mathrm{U}}
$$


has been shown to take values of 0.1-0.5 depending on 1) the geometry of the bluff body, 2) its aspect ratio (ratio of length spanwise direction to a characteristic length scale of its crosssection), 3) the Reynolds number of the flow,

$$
\operatorname{Re}=\frac{\rho U w}{\mu}
$$

4) the nature of the boundary layer separation upstream of the wake, and 5) the blockage ratio,

$$
\text { Blockage }=\frac{A_{\text {Bluff Body }}}{A_{\text {Duct }}}
$$

(the fraction of the duct's cross-section blocked by the bluff body) [11-12].

The character of the wake's flow conditions can be further characterized by the Roshko Number, $R o$, which is essentially the product of the Reynolds number and the Strouhal number, and describes the turbulence of the wake [6]

$$
\mathrm{Ro}=\frac{\rho \mathrm{fw}^{2}}{\mu}
$$

For cylinders in cross-flow, shedding frequencies have been shown to transition from approximately $\operatorname{Str}=0.21$ at Reynolds numbers below $10^{5}$ and then jump suddenly to approximately $\operatorname{Str}=0.5$ for $10^{5}<\operatorname{Re}<10^{6}$ and then fall back to around $\operatorname{Str}=0.26$ for $\operatorname{Re}>10^{6}$ [13]. It was also shown that this intermediate shedding regime could be effectively removed, that is transition directly from the lower Re regime to the higher, by artificially roughening the bluff body [11].

Where this work features a bluff-body confined in a narrow duct, for non-reacting flows, relevant work has shown that the bluff body's size relative to the size of the duct's major and minor cross-section dimensions can affect the frequency of the shedding of the vortices and the distance downstream of the bluff-body that the coherent structures will be formed [12]. Consistent with other works on Str variation with aspect ratio, the findings indicate that endwall 
effects reduce Str. The work reported currently is unique even to the ranges presented by Huang in offering results for a very low aspect ratio flameholder.

\subsubsection{Proper Orthogonal Decomposition}

Proper orthogonal Decomposition (POD) as it referred to here, is a data analysis technique originally proposed in 1901 [14] and first recognized by Lumley [15] for its value in analyzing turbulent flows. The statistical technique has been applied to data in many different fields and to address a wide range of problems - from pollutant dispersion [16] to reduced-order modeling [17] to machine vision [18] to neurology [19]. Owing in part to the diversity of its applications, POD is also referred to by a number of names including: principal component analysis, the Karhunen-Loève transformation, the method of empirical orthogonal functions, singular-value decomposition, eigenvalue decomposition, factor analysis, the Eckart-Young theorem, empirical component analysis, the Hotelling transform, among others. The method is essentially a pattern recognition technique that seeks to approximate a dataset through a linear combination of a minimum number of orthogonal vectors [20].

While limited in aspects in its ability to discriminate between data classes [21], POD's proven application in detecting coherent structures in turbulent [22-23] and reacting flows [2425], together with its agnosticism toward the source of data, make this technique particularly well-suited to the comparison of simulated and measured unsteady flow and combustion dynamic data.

The method for calculating the POD begins by compiling a "snapshot' of sample data, either measured or simulated, into column vector $\mathbf{u}^{\mathbf{i}}$ in equation 1.5 , these vectors are then assembled into an MxN matrix $\mathbf{U}$ in equation 6 where $M$ is the number of samples in each snapshot and $\mathrm{N}$ is the number of snapshots .

$$
u^{i}=\left[\begin{array}{c}
u_{1}^{i} \\
u_{2}^{i} \\
u_{3}^{i} \\
\vdots \\
u_{m}^{i}
\end{array}\right]
$$




$$
\mathrm{U}=\left[\begin{array}{lllll}
u^{1} & u^{2} & u^{3} & \cdots & u^{N}
\end{array}\right]
$$

Each element of the matrix $\boldsymbol{U}$ is then subtracted by the mean of its row [26]. The correlation matrix $\widetilde{\boldsymbol{C}}$ is calculated by premultiplying $\boldsymbol{U}$ by its transpose and dividing by N. Next the eigenvalue problem of $\widetilde{\boldsymbol{C}}$ is solved to find the vector of eigenvalues, $\lambda$, and the matrix of eigenvectors, $\boldsymbol{B}$. These are then multiplied to recover the matrix of unweighted eigenmodes $\boldsymbol{P}$. The properly weighted eigenmodes are calculated by multiplying each unweighted eigenmode with its corresponding eigenvalue.

Where POD is often used to construct a reduced-order model of a system by summing the most energetic modes, there are essentially two applications in the present work, both of which treat each mode (or pair of modes in the case of travelling waves which form sin/cosine pairs) as an isolated physical phenomenon which represents a coherent structure in the data. First, each structure can be used as a means of system identification to qualitatively characterize the system. Second, the properly weighted modes can form the basis for quantitative (in both magnitude and

spatial distribution) comparison between similar phenomena isolated from decompositions of different datasets to determine to what extent the two systems share a mode of given dynamic behavior.

\subsubsection{Frequency Spectra of POD Coefficients}

Given that each eigenmode is a linear combination of the ensemble of snapshots, and that the eigenvectors of each mode yield the weighting coefficients for the linear combination, and assuming the chronologically ordered snapshots have been sampled at regular intervals in time, then each eigenvector can be seen of as a time-series containing spectral information. Plotting the frequency spectra of these modes can give insight into the frequencies associated with a given mode.

\subsubsection{Extended POD}

Another application to the POD method is that of extended POD (EPOD), where the POD modes calculated from one set of data are projected onto a second data set that was sampled simultaneously with the first [27-28]. This is done by using the eigenvalues of the 
original POD to act as the linear weighting coefficients $\boldsymbol{B}_{\mathbf{1}}$ for the second dataset $\boldsymbol{U}_{\mathbf{2}}$ to form a new set of eigenmodes.

$$
\mathrm{P}_{2}=\mathrm{U}_{2} \mathrm{~B}_{1}
$$

This provides a visualization of the amount of correlation between two variables which may be temporally and spatially correlated (e.g. pressure and velocity). This technique has been applied to the correlation between velocity and heat-release fluctuations in both experimental [24] and numerically simulated [29] flames.

\subsubsection{Non-reacting Large Eddy Simulation Method}

The method of Large Eddy Simulation of fluid flows has been an active area of research since it was first proposed 50 years ago [30]. The underlying principle remains the same however, namely that the Navier-Stokes equations that govern fluid flow can be split between those scales of motion that can be adequately resolved by the discrete numerical grid used for the simulation, and those that cannot. The variables that are resolved by the grid are referred to as the filtered variables and the effect of the fluid motions too small to be captured directly is modeled in the equations of motion of the filtered variables as shown in the filtered continuity, momentum, and energy equations shown below as equations 1.8-1.11. Finally, two equations of thermodynamic relationships between the transported variables, in this case the ideal gas law, equation 10 and the calorically perfect gas assumption equation 1.12 .

$$
\begin{gathered}
\frac{\partial \bar{\rho}}{\partial t}+\nabla \cdot(\bar{\rho} \overrightarrow{\vec{u}})=0 \\
\frac{\partial \bar{\rho} \overrightarrow{\vec{u}}}{\partial t}+\nabla \cdot(\bar{\rho} \overrightarrow{\bar{u}} \overrightarrow{\vec{u}})=-\nabla \bar{P}+\nabla \cdot\left(\sigma_{i j}\right) \\
\frac{\partial \bar{\rho} \bar{e}}{\partial t}+(\overrightarrow{\vec{u}} \cdot \nabla) \bar{e}=\nabla \cdot\left(-\bar{\rho} \overrightarrow{\bar{u}} \bar{P}+\bar{\rho} D_{t h} \nabla \bar{e}\right)+\dot{q} \\
\bar{P}=\bar{\rho} R \bar{T} \\
\bar{e}=c_{v} \bar{T}
\end{gathered}
$$


Clearly the success of any LES approach will be dependent on the how well the subgrid scale physics are modelled. In this work, the SGS models used in the momentum equations are all adapted from those originally proposed by Smagorinsky, which invokes the Boussinesq hypothesis, namely that the effect of the eddy motion can be modeled as an increased turbulent viscosity which is added to the molecular viscosity of the working fluid. Smagorinsky's SGS model, given in equation 1.13, models this viscosity as proportional to the magnitude of the local resolved strain rate, $\dot{\sigma}$, as calculated from then filtered velocity field, and proportional to the square of the filter width, $\Delta$, which is calculated as the cube-root of the volume of the computational shell as shown in equation 1.14. From this model the shear stress tensor can be calculated, as in equation 1.15, demonstrating that the turbulence model, at least for the momentum equation is now closed.

$$
\begin{gathered}
\mu_{\Delta}=\bar{\rho}\left(C_{s} \Delta\right)^{2}|\dot{\sigma}| \\
\Delta=\sqrt[3]{V_{\text {Cell }}} \\
\bar{\tau}_{i j}=\mu_{\Delta}\left[\frac{\partial \bar{u}_{i}}{\partial x_{j}}+\frac{\partial \bar{u}_{j}}{\partial x_{i}}-\frac{2}{3} \frac{\partial \bar{u}_{k}}{\partial x_{k}} \delta_{i j}\right]
\end{gathered}
$$

The value of $\mathrm{C}_{\mathrm{S}}$ in equation 1.12, can be specified in two ways, either as a constant as it was originally proposed, usually taken to be roughly 0.1 , but which may require tuning to an optimal problem-specific value as comparisons with DNS data have shown that $\mathrm{C}_{\mathrm{S}}[31]$. In an attempt to eliminate the need for tuning and to remove the constraint of a having to define a priori a single globally-constant coefficient, Germano proposed a dynamic version of Smagorinsky's model which defines the $\mathrm{C}_{\mathrm{S}}$ coefficient based on local conditions [32-33].

To ensure that the subgrid scale model is not responsible for representing more of the turbulent kinetic energy than can be justified from the assumption originally proposed for the LES approach, it has been recommended that at least $80 \%$ of the local turbulent kinetic energy be captured by the resolved scales and that no more than $20 \%$ should be modeled by the sub-grid scale model. To quantify this, the time-average of the local subgrid kinetic energy, as calculated in equation 1.16, is compared to the resolved turbulent kinetic energy from a traditional Reynolds decomposition, defined in equation 1.17. Finally, the Pope criterion M, which as 
mentioned previously should be no more than 0.2 in the regions of interest, is calculated with equation 18.

$$
\begin{gathered}
\overline{k_{\text {sgs }}}=\overline{\left(\frac{\mu_{\Delta}}{C_{S} \Delta}\right)^{2}} \\
k_{\text {resolved }}=\overline{u_{l l}^{\prime}} \\
M=\frac{k_{\text {sgs }}}{k_{\text {sgs }}+k_{\text {resolved }}}
\end{gathered}
$$

\subsection{EXPERIMENT}

This section describes the facilities used for conducting the experimental portion of this work including the laboratory infrastructure, the analyses that were completed as part of the design of the rig, a summary of the design features that enabled the measurement techniques described later, and several characterizations of the conditions in the test section.

\subsubsection{CSDL Facilities}

The experiments described here took place in a newly-constructed test rig that was built for evaluating augmentor flameholder concepts and developing diagnostic techniques to measure the physical phenomena of interest in augmentor performance. The new augmentor development rig was built as part of the modular combustion rig housed in the Combustion Systems Dynamics Lab (CSDL) at Virginia Tech which has a background of experimental work in combustion dynamics [34], laminar and turbulent premixed combustion [35], multiphase flow [36] and optical diagnostics [37]. The lab features a Kaeser FS440 air compressor capable of delivering up to $0.7 \mathrm{~kg} / \mathrm{s}(1.5 \mathrm{lbm} / \mathrm{s})$ of air at up to $10 \mathrm{~atm}(150 \mathrm{psia})$. The lab is also equipped with electrical heaters which preheat air with unvitiated air at temperatures up to $650 \mathrm{~K}(700 \mathrm{~F})$. After heat loss and compressibility effects, the envelope of possible inlet temperatures and bulk-flow Mach numbers is shown in figure 1.2. 


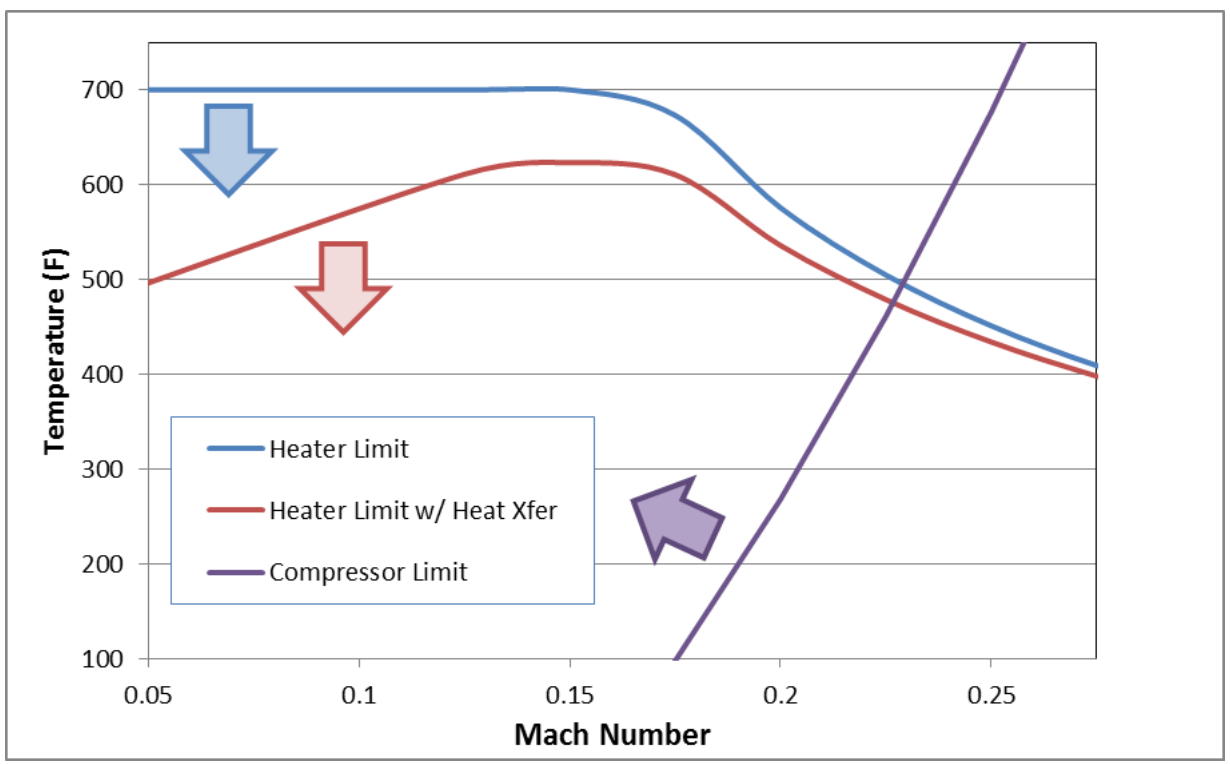

Figure 1.2 - Test section temperature and Mach number limitations from facility constraints.

\subsubsection{Rig Layout and Design}

The augmentor development rig was built as part of the modular combustion rig at the CSDL, the components, shown in figure 1.3, consists three primary components: a fuel-injection section, a converging section, and the test section.

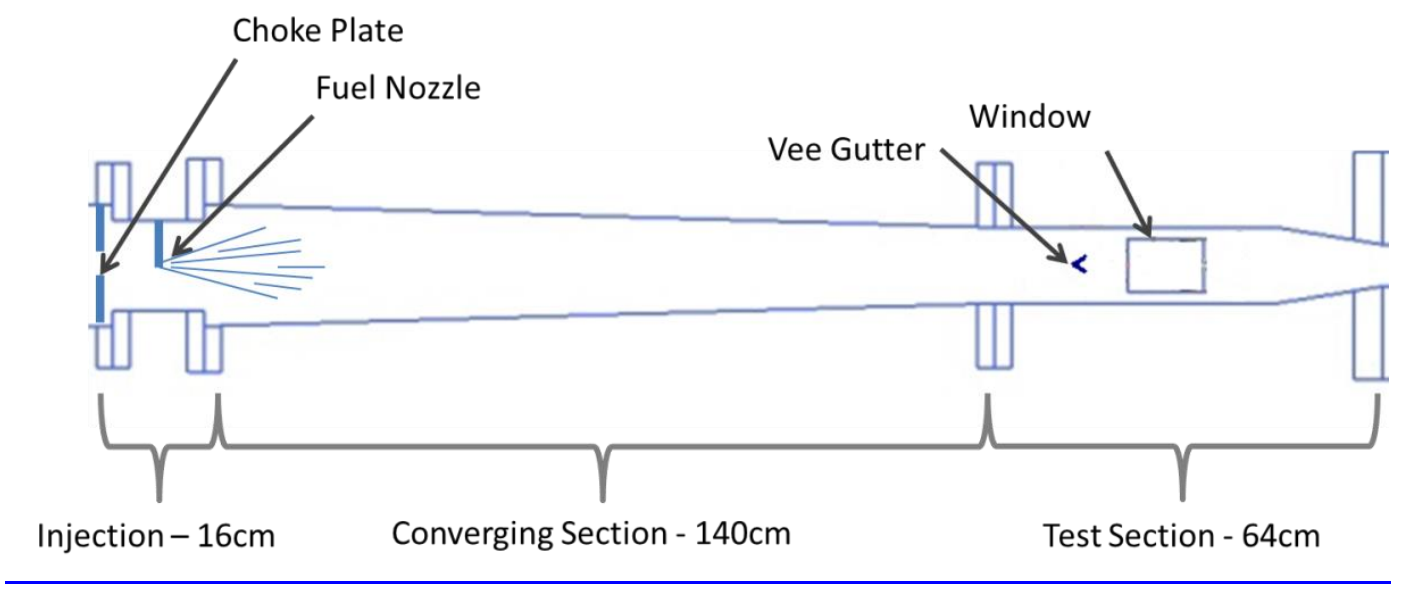

Figure 1.3 - Schematic of experiment showing the three sections of the rig and the locations of the fuel injection, vee-gutter flameholder, and windows. 
The test-section features a $12.7 \mathrm{~cm} \times 5 \mathrm{~cm}\left(2 " \times 5\right.$ ") rectangular cross-section, a $70^{\circ} 3 \mathrm{~cm}$ (1.2") wide vee-gutter flameholder which horizontally spans the minor length of the crosssection, film-cooled windows for optical access just downstream of the flameholder, a series of access points along the centerline of the lower wall of the test-section, and a water-jacket downstream of the windows. The flameholder is held in place by two removable plates, which clamp the sides of the flameholder and prevent it from slipping.

Upstream of the test-section is a converging section $140 \mathrm{~cm}\left(55^{\prime \prime}\right)$ in length that transitions the flow from a circular $20 \mathrm{~cm}(8 ")$ diameter cross-section to the rectangular cross-section of the rig's test-section. Immediately upstream of the converging section is the injection section where fuel and seed particles are injected. Just upstream of the injection section is a choke plate which acoustically isolates the experiment from the rest of the air supply. The rig is designed primarily to burn liquid fuels but gaseous fuels have been used as well. In either case the fuel is injected sufficiently far upstream of the flameholder that the fuel and air are fully mixed when they reach the flameholder and are burned. The fuel delivery systems is capable of supplying $7.5 \mathrm{lpm}(2$ gpm) of liquid fuel or the stoichiometric equivalent of natural gas to the single simplex atomizer which is used to atomize the fuel and spray it into the air stream.

\subsection{Vee-Gutter Flameholder}

While the test rig was designed to accommodate multiple flameholder configurations including bluff-body and jet-stabilized flameholders, the work presented here focuses solely on a sharp-nosed vee-gutter type bluff-body flameholder. The vee-gutter has a constant, vee-shaped cross-section and is oriented to span the $50.8 \mathrm{~mm}$ ( $2.0 \mathrm{in}$ ) minor dimension of the test section (that is it spans the flow horizontally and perpendicular to the flow). To achieve the desired blockage ratio of 0.24 the vee-gutter was designed to have a tip-to-tip distance of $30.5 \mathrm{~mm}(1.2$ in) and its included angle was chosen to be 70 degrees so as to be representative of typical afterburner configurations [38].

\subsection{Optical Access}

The test-section features three windows, a pair of side windows that are $9 \times 13 \mathrm{~cm}(3.5 \mathrm{x}$ 5 inches $)$ in size and a single top window $2.5 \times 13 \mathrm{~cm}(1 \times 5$ inches $)$ in size. The windows, shown in figure 1.4, are designed to allow for the use of multiple diagnostic measurement techniques including stereo particle image velocimetry (PIV), Rayleigh and Raman scattering, and 
absorption spectroscopy which are described in depth later in the paper. To mitigate the risk of overheating the windows and fouling them with entrained PIV particles, the windows are filmcooled. The windows are also designed as bolt-on modules that can be easily removed to minimize downtime when the windows do require cleaning.

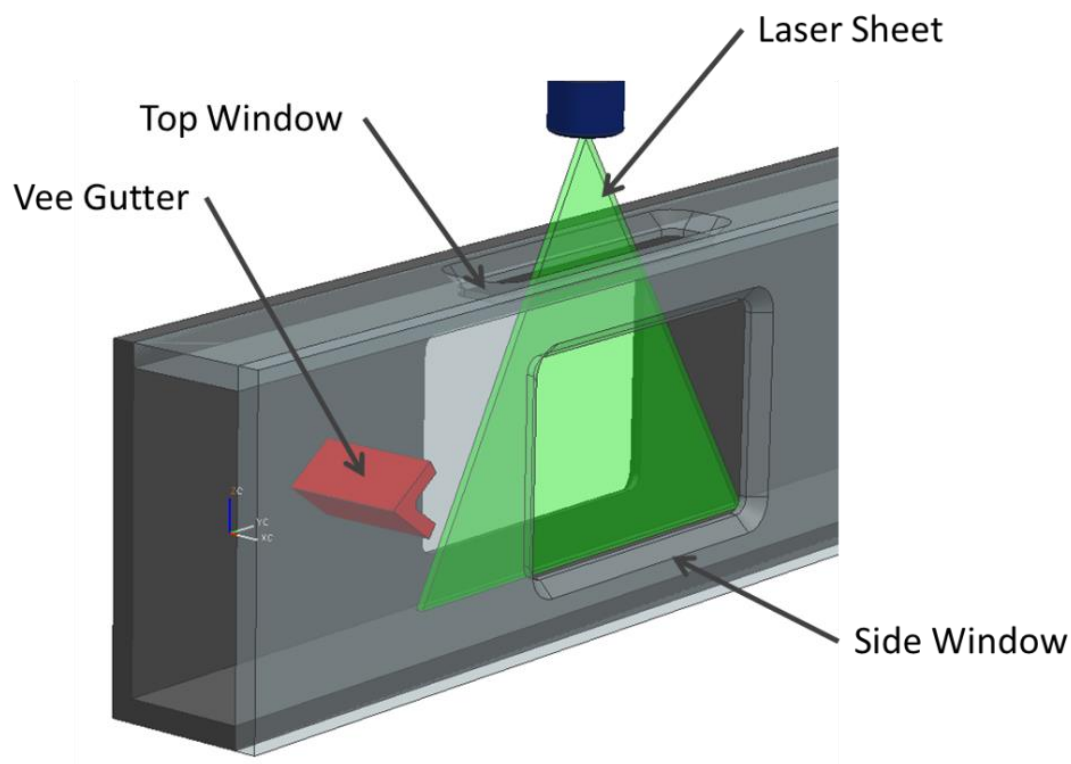

Figure 1.4 - Transparent view of CAD model of the test section showing the locations of the three windows relative to the vee-gutter flameholder (and the PIV laser sheet).

\subsubsection{PIV}

PIV is used to acquire instantaneous velocity information in a flow plane. Using stereoscopic PIV, three dimensional vector fields can be produced by using two cameras with separate viewing angles. By calibrating and focusing both cameras on the same plane of interest, the images of the illuminated particles can be resolved into vector fields with all three components of velocity.

A LaVision Flowmaster PIV system, including both hardware and software, was used to make the measurements reported here. The system consists of two cameras, each with a resolution of $2048 \times 2048$. The download time from the cameras to the system computer is such that the maximum continuous sampling speed is limited to approximately 2 samples per second. When using the cameras' onboard memory to buffer between downloads the sampling time can 
be as high as $7 \mathrm{~Hz}$ over a 5 second window before the memory must be downloaded. The particles were illuminated by a dual-cavity frequency-doubled $532 \mathrm{~nm} \mathrm{Nd:YAG} \mathrm{laser.} \mathrm{The} \mathrm{DaVis}$ 7 software provided by LaVision was used for initial pre-processing of the images, for compiling the autocorrelations to recover the fields of velocity vectors, and for some post-processing of the vector fields to remove spurious data. Seeding of the flow was accomplished by injecting $1 \mu \mathrm{m}$ $\mathrm{TiO}_{2}$ particles dispersed using a swirling fluidized bed seeder and injected through a simple tube in a port in the rig's converging section located $30 \mathrm{~cm}$ upstream of the test section.

\subsection{LARGE EDDY SIMULATION}

A number of LES models were built using ANSYS FLUENT and OpenFOAM to simulate the flow in the test section under non-reacting conditions. The boundary conditions for these simulations as well as the computational domain and solver settings will be described here.

\subsubsection{Boundary Conditions}

The simulations performed for this work were built to match a specific experimental run for which the best PIV data was available. The first case is non-reacting flow where experimental measurements had been made of the velocity in the vee-gutter's wake using PIV for instantaneous two-dimensional snapshots of 2-component velocity vectors as well as a Pitot probe for time-average streamwise velocity profiles which were used to measure in some locations not accessible to the PIV cameras' fields of view. The boundary conditions for this case consist of mass-flow, inlet temperature, and ambient pressure (assumed at the outlet) are shown in table 1.1.

Table 1.1 Boundary conditions for the simulations reported.

\begin{tabular}{|c|c|c|}
\hline Parameter & Value & Units \\
\hline Inlet Mass Flow Rate & 0.283 & $\mathrm{~kg} / \mathrm{s}$ \\
\hline Inlet Temperature & 298 & $\mathrm{~K}$ \\
\hline Outlet Pressure & 95 & $\mathrm{kPa}$ \\
\hline
\end{tabular}




\subsubsection{Domain and Mesh}

The computational domain used for all of the simulations shown here consisted of a constant rectangular cross-section duct and a prismatic $70^{\circ}$ degree included angle vee-gutter, with a $3.05 \mathrm{~cm}$ tip to tip measurement ' $w$ ', extending through the minor axis of the duct. The veegutter has a thickness of $0.21 \mathrm{w}$. As shown in figure 1.5, the domain extends $9.1 \mathrm{w}$ upstream of the vee gutters leading edge which is actually longer than the test section itself however this length was chosen based on boundary layer analysis to account for the boundary layer in the transition section that exists prior to the flow entering the test section. The domain is $24.1 \mathrm{w}$ long overall, as shown in figure 1.6, and maintains a rectangular $1.67 \mathrm{w} \times 4.17 \mathrm{w}$ cross-section throughout. The real experiment also has a constant cross-sectional area, but begins to transition to a hexagonal cross-section at the end of the water-jacket section, this geometrical difference was assumed negligible.

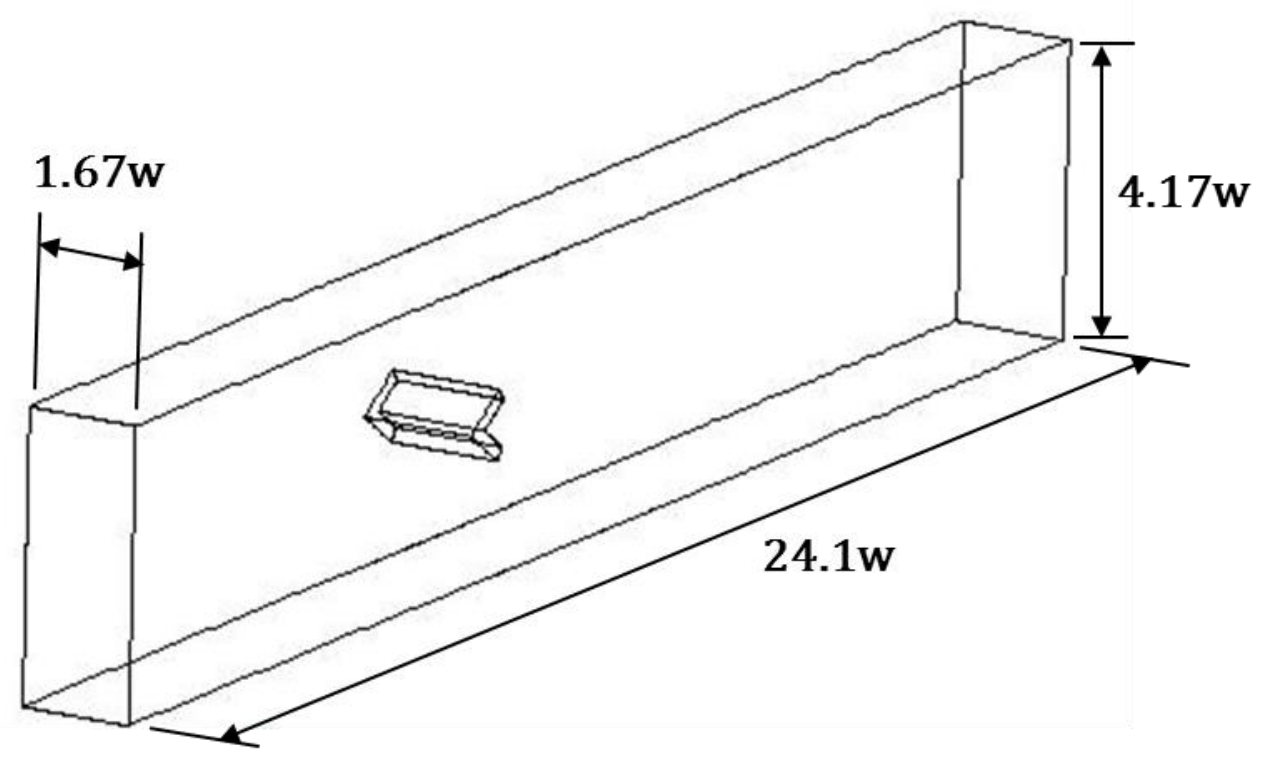

Figure 1.5 - Isometric view of dimensions of the domain used in the LES models based on the width of the flameholder $\mathrm{w}=3.05 \mathrm{~cm}$. 


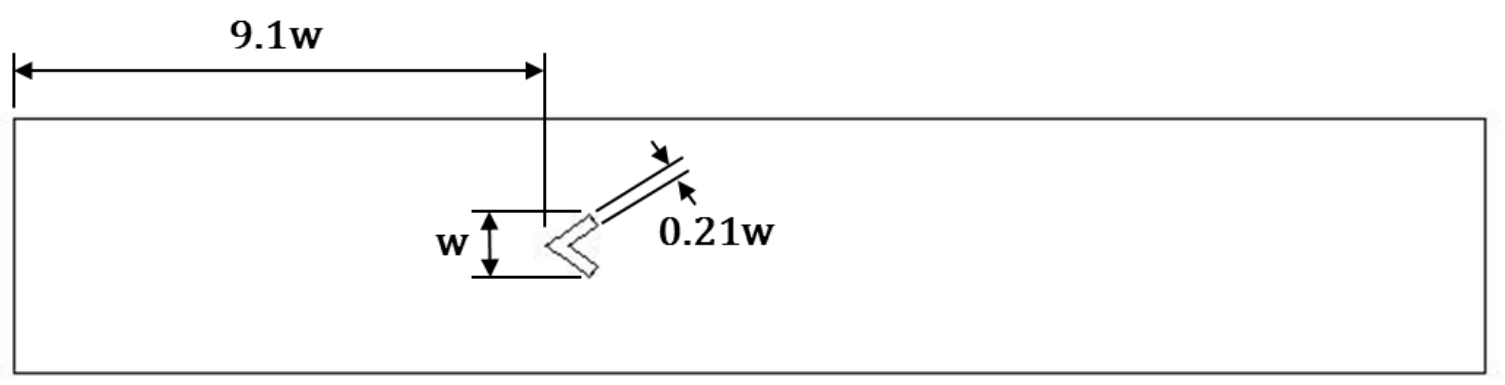

Figure 1.6 - Plan view dimensions of the domain used in LES models, based on the dimension of the vee-gutter flameholder $\mathrm{w}=3.05 \mathrm{~cm}$ which has an included angle of $70^{\circ}$,

Two meshes were used in these simulations, one with $2.8 \mathrm{M}$ cells served as the baseline while a finer mesh was run for some cases; this mesh was a $2 \times 2 \times 2$ refinement of the baseline mesh with 22M cells. The number of cells used in the baseline mesh was chosen for two reasons. First based on the available 16-core workstation used in this work which was limited to 96GB of RAM, the refined mesh with $22 \mathrm{M}$ cells just fit under the limit using 90GB of memory for the reacting cases to run in Fluent. Second, the refined mesh cases and took roughly a month and a half each to compute, with the same computational resources the cases on the baseline mesh could each be completed in three to four days.

The meshes were built in a block-structured format using ICEM Hexa and then converted to the unstructured formats used by the two solvers. In making the mesh, care was taken to limit the growth rate in any direction to 1.05 or smaller and the maximum aspect ratio of any cell in the region of interest was limited to 5 or less. Downstream of the region of interest, the aspect ratios of the cells were intentionally stretched in the streamwise direction to dampen out fluctuations in the flow before they reached the outlet boundary.

\subsubsection{Comparison with Experiment}

For comparison between to the PIV data, the CFD solutions were sampled from a 100x100 grid of interrogation locations located on the midplane of the test section and extended 
downstream from the trailing edge of the vee-gutter as shown in figure 1.7. The solutions were sampled every $5^{\text {th }}$ simulation timestep.

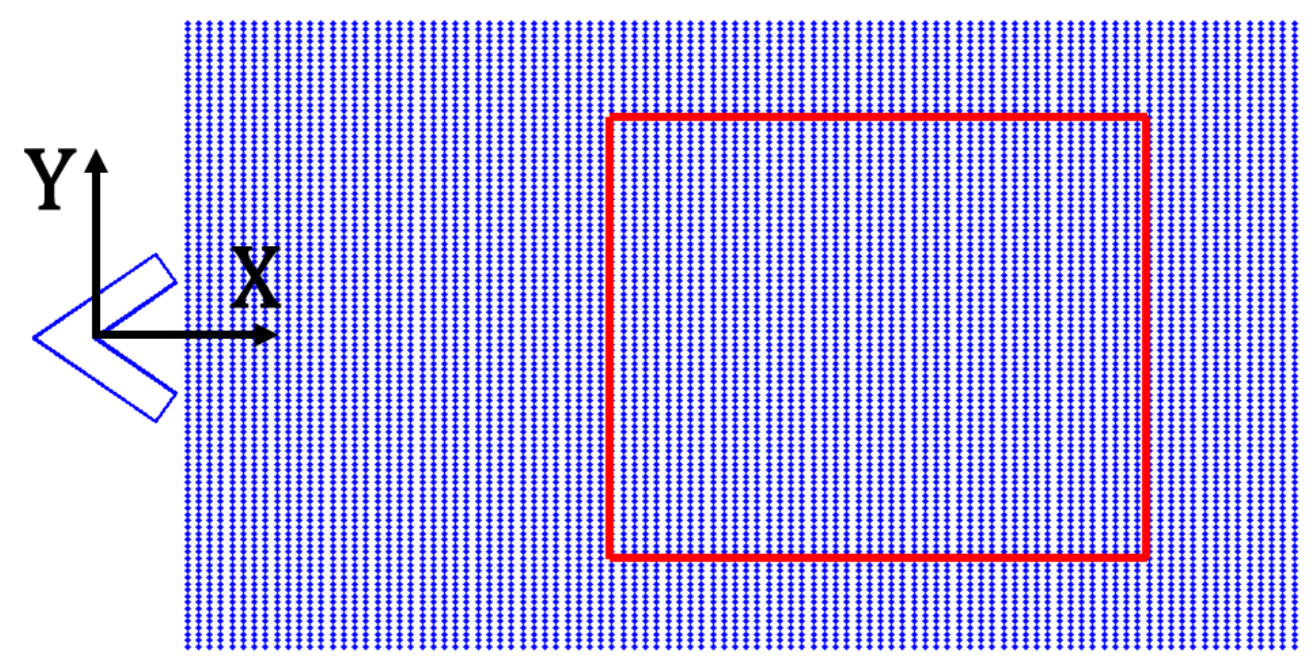

Figure 1.7 - Illustration of relative sizes and locations of sampling planes relative to the flameholder: 100x100 CFD sample grid points (blue) compared to the effective viewing area for the PIV measurements.

\section{$1.7 \quad$ RESULTS}

A number of simulations were conducted to investigate the effect of various physical and numerical factors on the wake's structure. The investigations began by comparing the time averages of the baseline simulation against the same data from the baseline simulation. The baseline simulation was run on the previously-discussed mesh using FLUENT 14 using the dynamic Smagorinsky sub-grid scale model. The time averaged profiles of streamwise velocity at $\mathrm{x} / \mathrm{w}=2.2$ and $\mathrm{x} / \mathrm{w}=3.8$ showed good agreement between the two data sets (figure 1.8). 

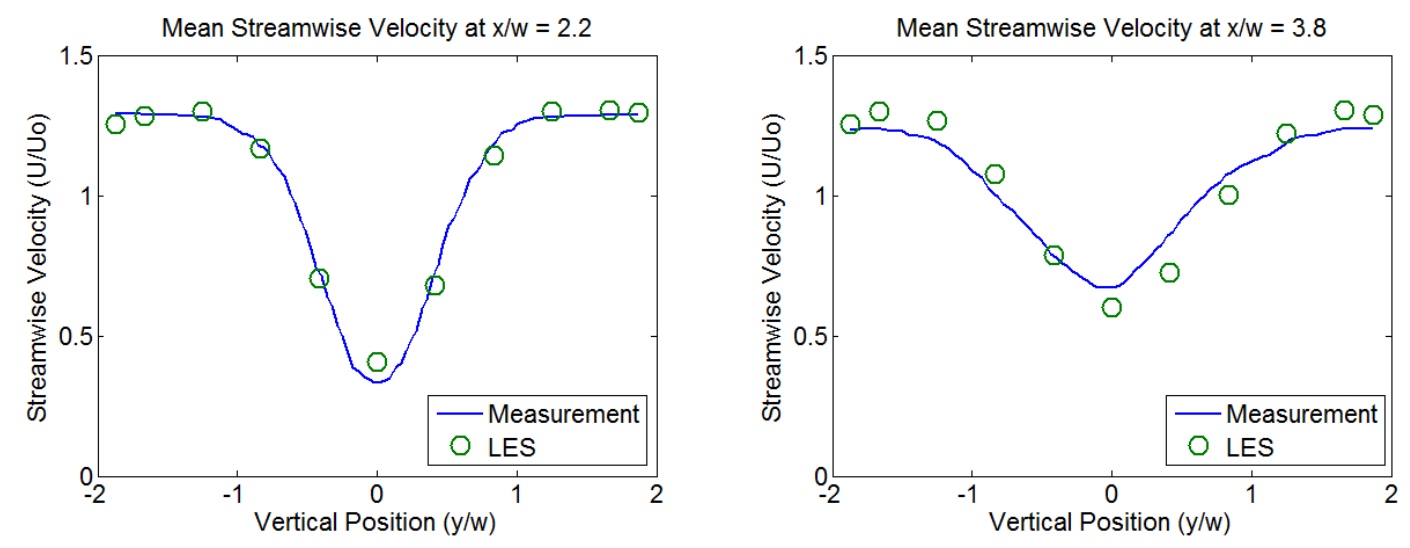

Figure 1.8 - Mean streamwise velocity plots as measured by Pitot probe and as simulated by Fluent LES.

The power spectral density of the $v$-velocity component at $\mathrm{y} / \mathrm{w}=0$ and $\mathrm{x} / \mathrm{w}=4$ (figure 1.9 ) exhibits a peak associated with shedding at approximately $S t r=0.2$. Also shown in this figure is the characteristic $\mathrm{f}^{-5 / 3}$ inertial subrange behavior typical of turbulent spectra.

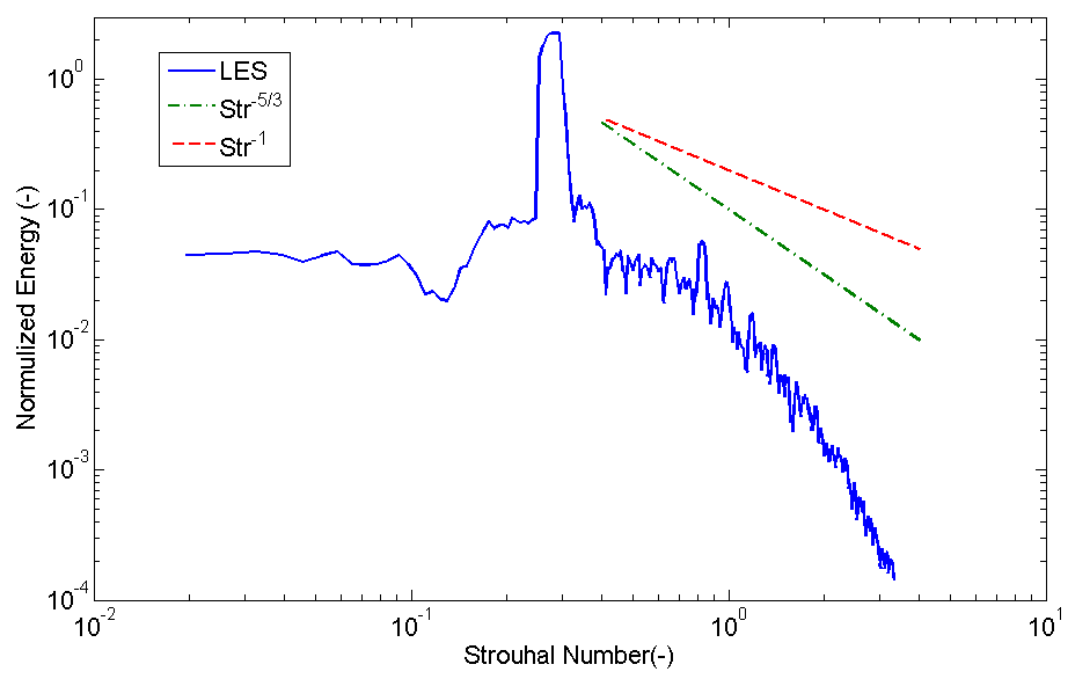

Figure $1.9-v^{2}$ Reynolds stress spectra spectrum from LES model. Note the peak due to vortex shedding at $S t r=0.24$ and also the decay rate that seems to correspond to the $-5 / 3$ power law rule. 


\subsubsection{Comparison of First Three Modes}

To compare the dynamics of the experimental and numerical experiments, the sum of the first three modes from the PIV data was compared to those of the baseline simulations in Fluent and OpenFOAM. The size and shapes of the oscillations captured by these modes showed good overall agreement between those visible in the PIV camera's field of view, which showed a single oscillation of the shedding mode, with those of the two numerical simulations as can be seen in figure 1.10, which show the size and location of the PIV window.
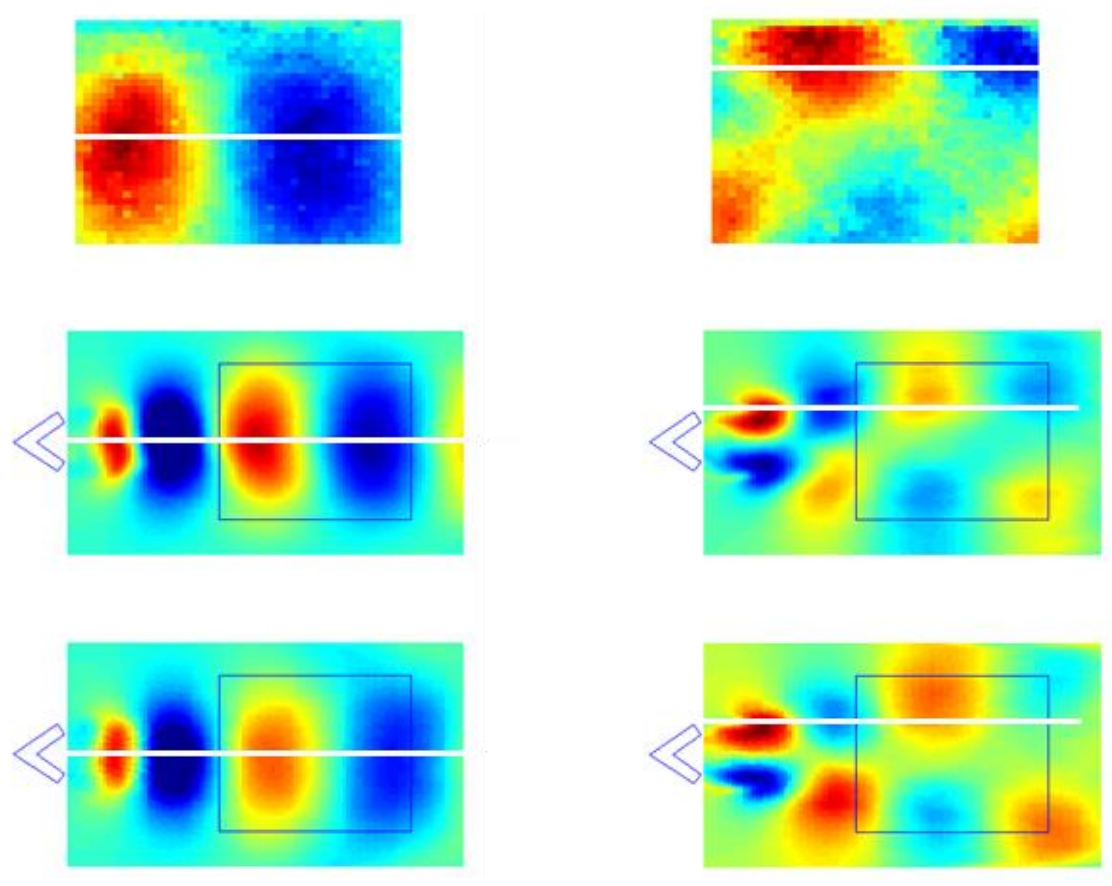

Figure 1.10 - Contours of the sum of the first three POD modes (left: v', right: u') obtained using results from PIV (top), Fluent (middle), and OpenFOAM (bottom). The profiles shown in figure 1.11 are taken along the white lines show on each plot.

The contours of $\mathrm{v}$-velocity profiles along the $\mathrm{y}=0$ line (white lines in figure 1.10) extending downstream from the vee-gutter were taken from the sum of the first three modes and plotted on the same axes, as shown in figure 1.11 show good agreement between the three datasets. 

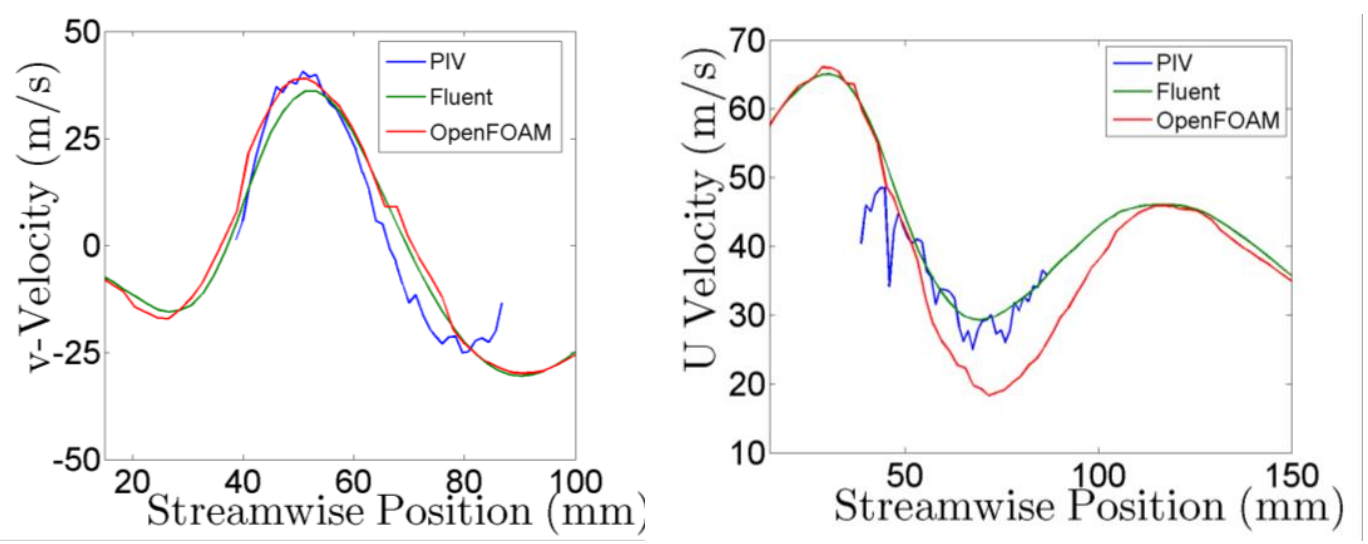

Figure 1.11 - Comparison between numerical and experimental values of the first three POD modes. Left: v', right: u'.

The comparison between the v-velocity components was followed by a similar comparison of the u-velocity of the sum of the first three modes of each dataset. The contours of this component of the modes, in figure 1.12, show good agreement among the three datasets between their sizes, shapes and relative magnitudes. These contours were sampled along the line $y / w=0.5$ to produce the profiles plotted on the same axis in figure 13 , which shows the Fluent simulation agrees more closely with the PIV data than the OpenFOAM simulation does. 

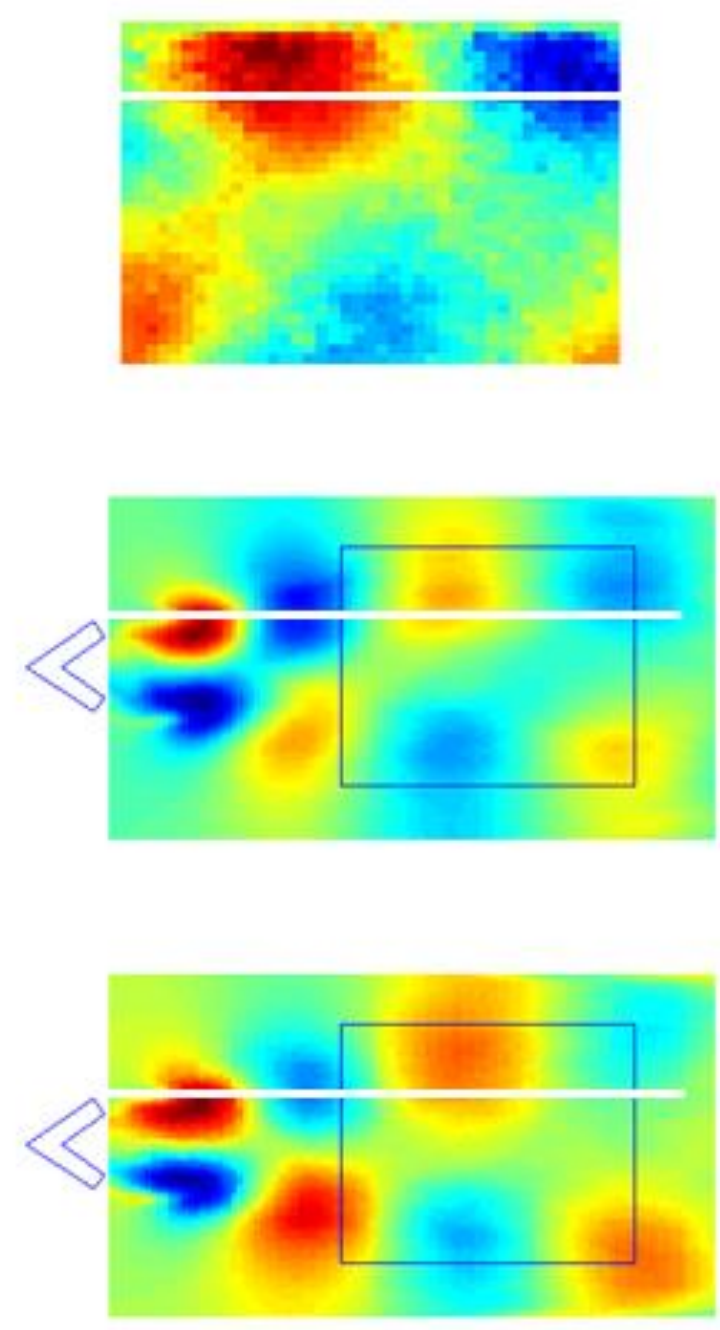

Figure 1.12 - Contours of u-velocity component of the sum of the first 3 POD modes of PIV (top), Fluent (middle), and OpenFOAM (bottom). The profiles shown in figure 13 are taken along the white lines shown imposed on each figure. 


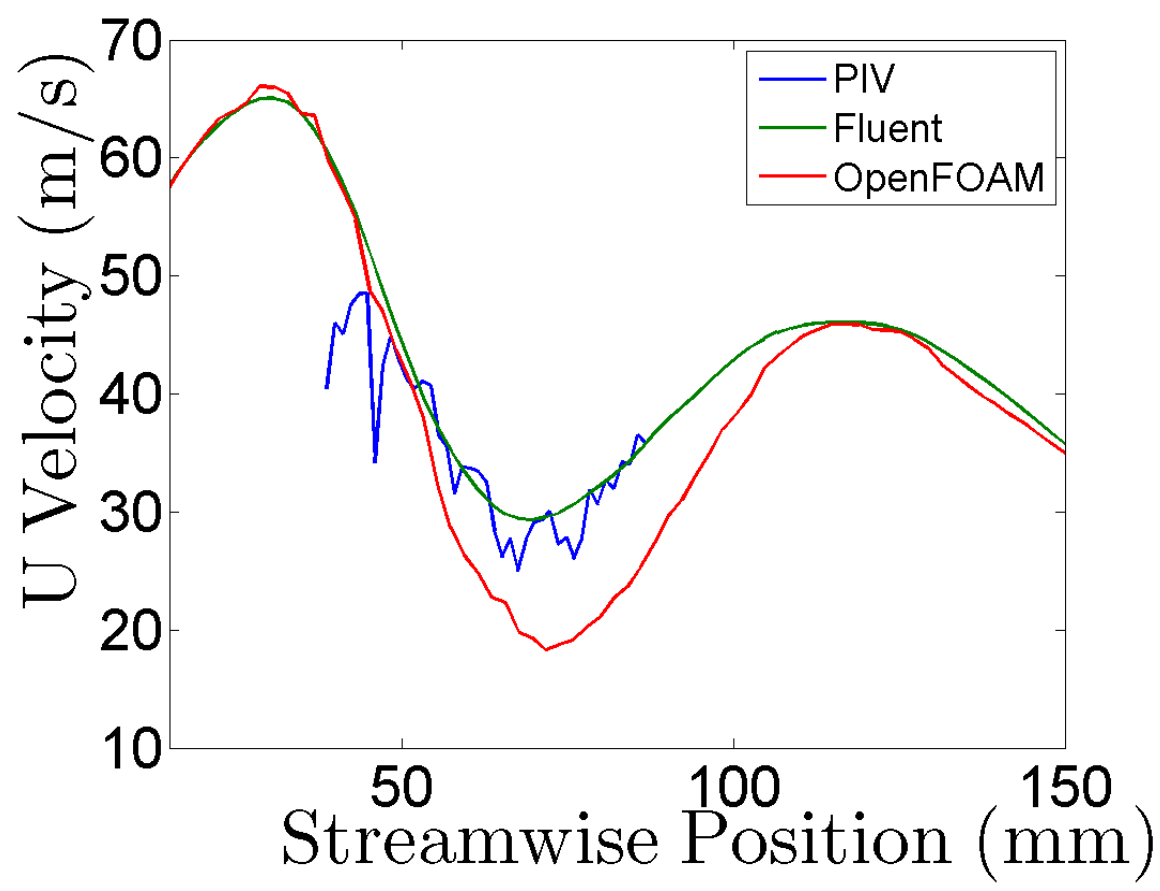

Figure 1.13 - Plot of u-velocity component of the sum of the first 3 POD modes taken along the lines indicated in figure 1.12.

\subsubsection{Near-wall Sampling and Extended POD}

Following this initial comparison, a number of additional investigations were completed to study the effect of various parameters on the dynamics of the wake's shedding mode. The first of these additional studies sought to understand the how the wake's dynamics were varied away from the midplane. The study consisted of sampling the simulation not just on the 100x100 point grid located at the midplane of the test-section as done previously, but to also sample the solution on an identical grid moved closer to the wall in the spanwise direction $80 \%$ of the distance to the wall. The v-component of the first three modes, shown in figure 1.14, show that the oscillations of the wake's shedding are about 7\% larger at the midplane than they are at this distance from the sidewalls. This observation was confirmed by using the so-called extended POD method to calculate the mode shapes of the nearwall dataset by using the coefficients obtained from the POD of the midplane dataset. This analysis, plotted in figure 1.14, also showed the same $7 \%$ 
difference in magnitude and no significant phase lag between the two waves, thus indicating a relatively 2-dimensional flow field without significant variation in the spanwise direction.

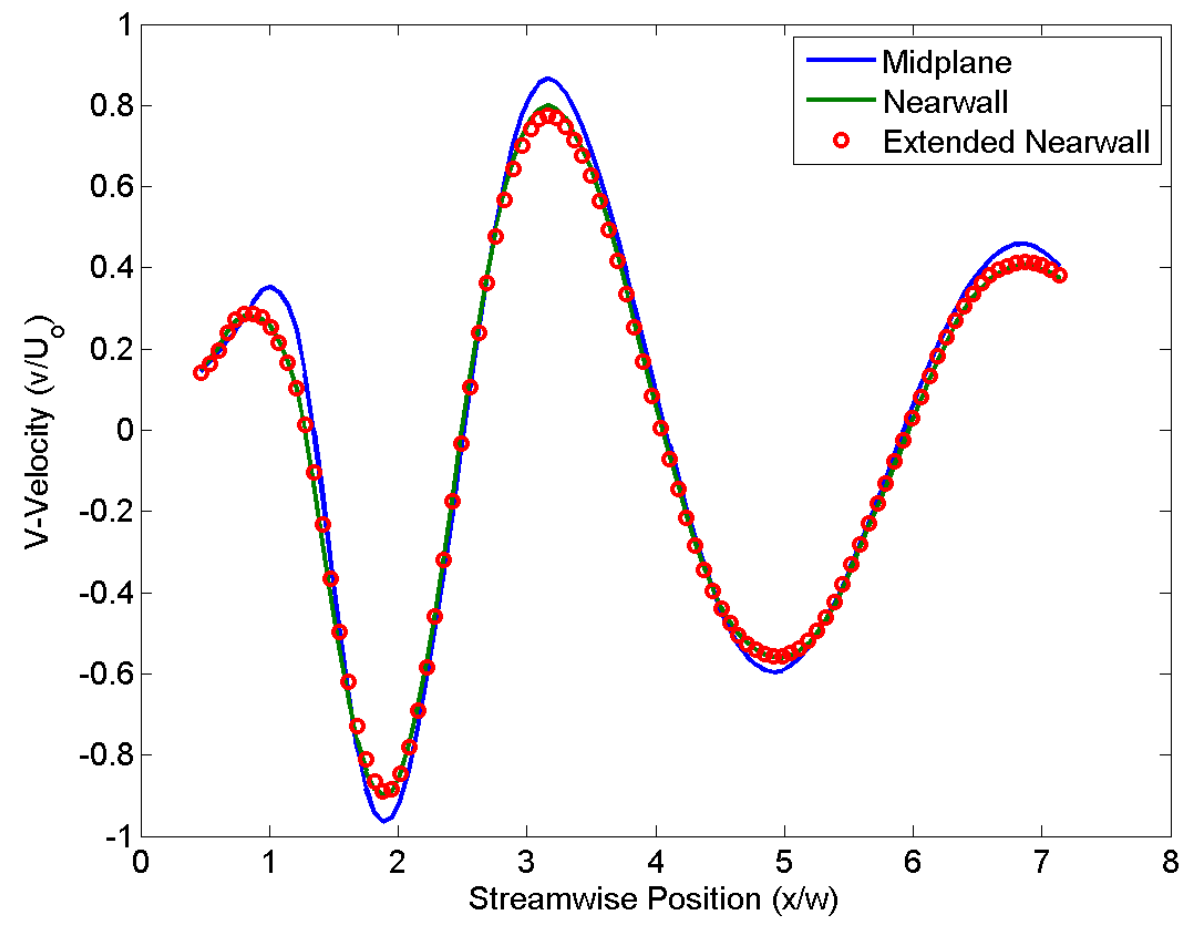

Figure 1.14 - Plot of $u$-velocity component of the sum of the first 3 POD as isolated at $y / w=0$ at the midplane, near the wall, and near the wall but with EPOD to recover the nearwall modeshapes using the eigenvectors of the midplane analysis.

\subsubsection{Boundary Layer}

To further study the effect the thickness of the boundary layer has on the wake's dynamics, the simulation's domain was extended such that the distance between the inlet and the vee-gutter increased from 9.1 vee-gutter widths to 14.1 (an additional distance of $152 \mathrm{~mm}$ ) as can be seen in figure 1.15 . 

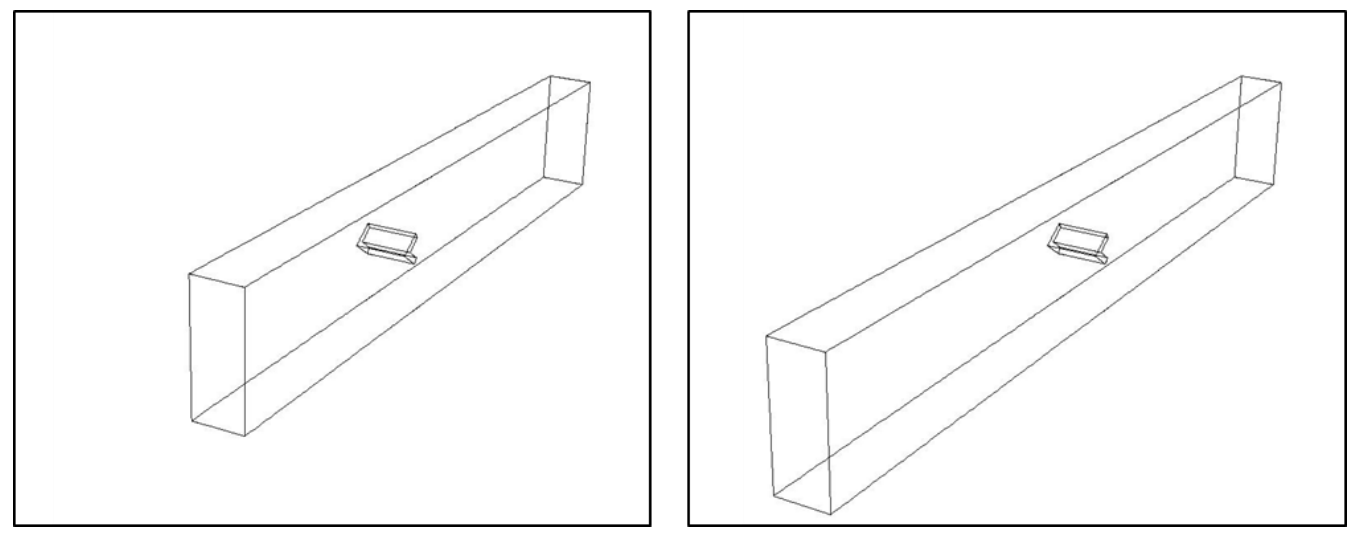

Figure 1.15 - Comparison between the baseline domain (left) and the domain with the extended domain (right) with increased distance between the inlet and the vee-gutter to produce a bigger/thicker boundary layer in the vicinity of the vee-gutter.

The wake dynamics in this configuration, again the sum of the first three modes of the simulation as isolated via POD, showed very similar behavior to that of the baseline simulation as can be seen in figure 1.16. When the profiles of the v-components of velocity of these modes are compared along the $\mathrm{y}=0$ line on the midplane, shown in figure 1.17 , some slight differences are evident. First, that the magnitude of the shedding oscillation just downstream of the veegutter is slightly higher in the case of the thicker boundary layer as might be expected since the increased displacement thickness of the bigger boundary layer would, by continuity, induce a slightly higher freestream velocity. Farther downstream however this trend is reversed and the oscillations in the baseline shedding are slightly larger than those in the case of the thicker boundary layer. 

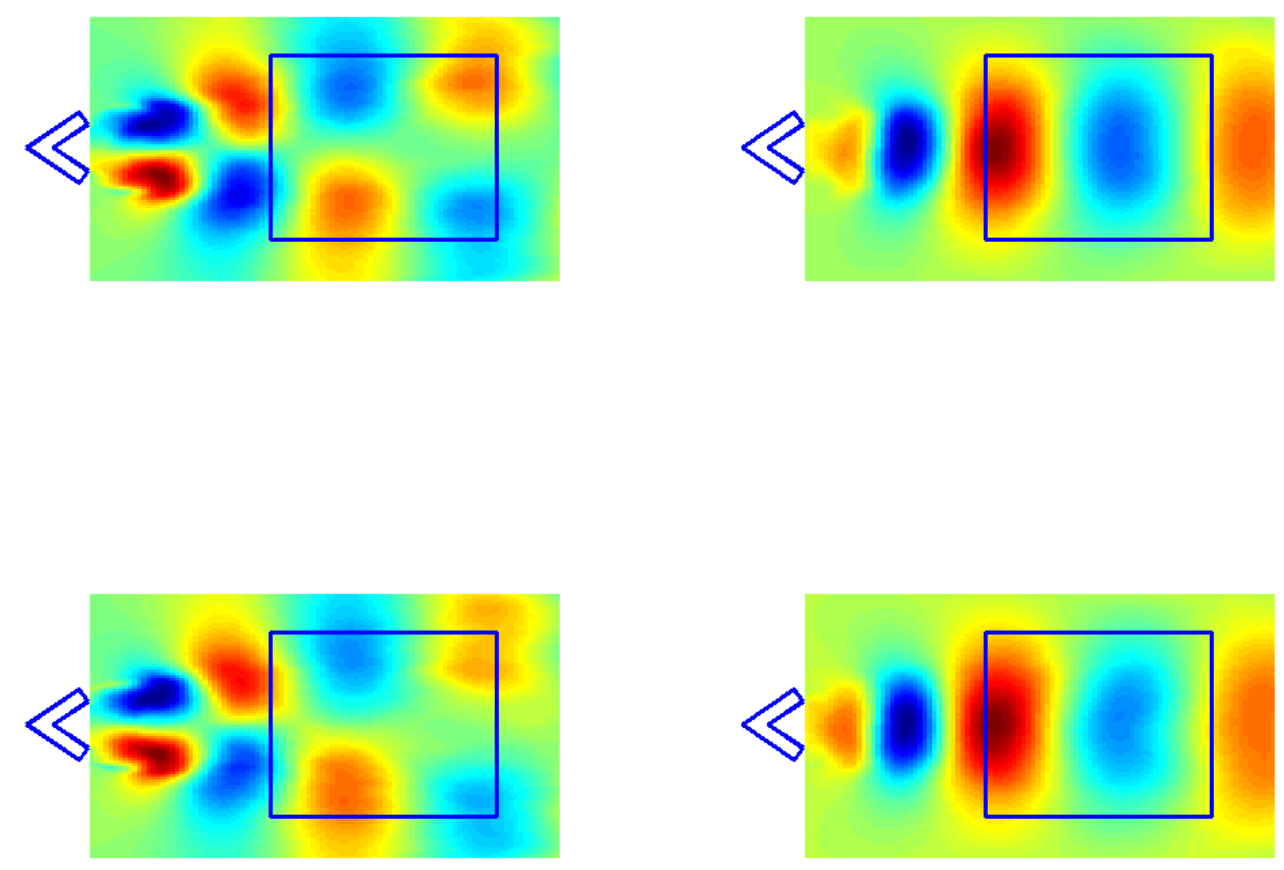

Figure 1.16 - Comparison between the sum of the first three POD modes of the baseline simulation (top) and thicker boundary layer configuration (bottom). Contours of the Ucomponent (streamwise) of velocity are shown on the left and V-component contours are shown on the right 


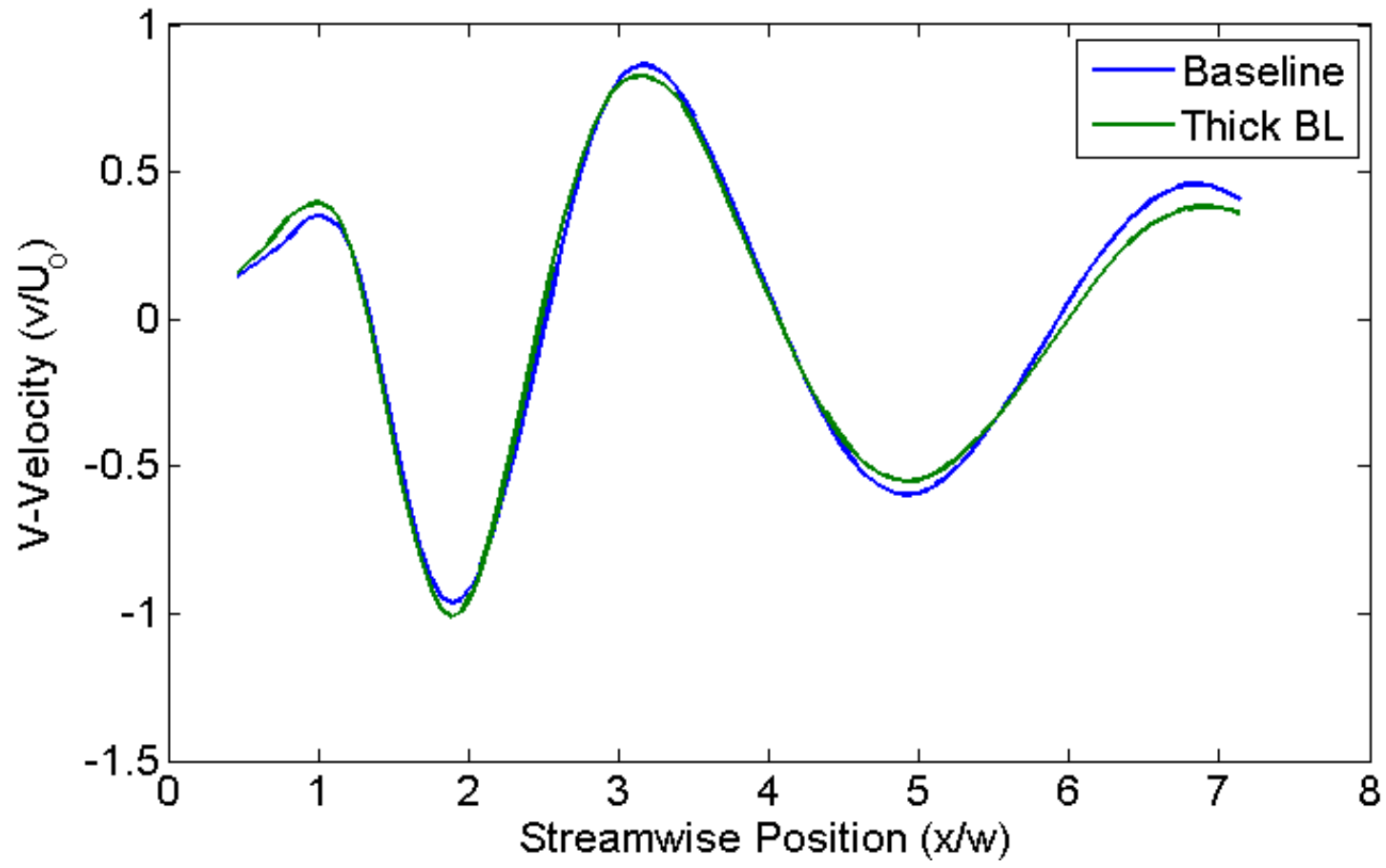

Figure 1.17 - Comparison of the v-components (vertical) of velocity of the sum of the first three modes of the baseline simulation and the configuration with the thicker boundary layer.

\subsubsection{Periodic Sides}

A third study, aimed at extrapolating the dynamics observed in the confined test section to a more typical vee-gutter configuration without sidewalls, consisted of changing the boundary conditions of the sides of the domain from no-slip wall boundaries to a pair of periodic boundaries. This eliminated completely any corner-flow effects at the junctions between the top and side walls or between the vee-gutter and the side walls. The analysis of the first three modes of this simulation was carried out in the same way the preceding examples. The results, again shown as contours of $\mathrm{u}$ - and v-velocity, are compared to the baseline simulation as shown in figure 1.18. These show little change in the sizes, shapes, or locations of the shedding mode from the baseline simulation but a comparison of the v-components of velocity along the $y=0$ line show two interesting, albeit slight differences. First, that the streamwise distances between the peaks of the oscillations are slightly larger for the case with periodic sides as compared to the baseline. Second, just downstream of the vee-gutter, the magnitude of the oscillations of the 
periodic case are larger than those of the baseline but then decay more rapidly such that they are nearly identical to the baseline by $\mathrm{x} / \mathrm{w}=7$.
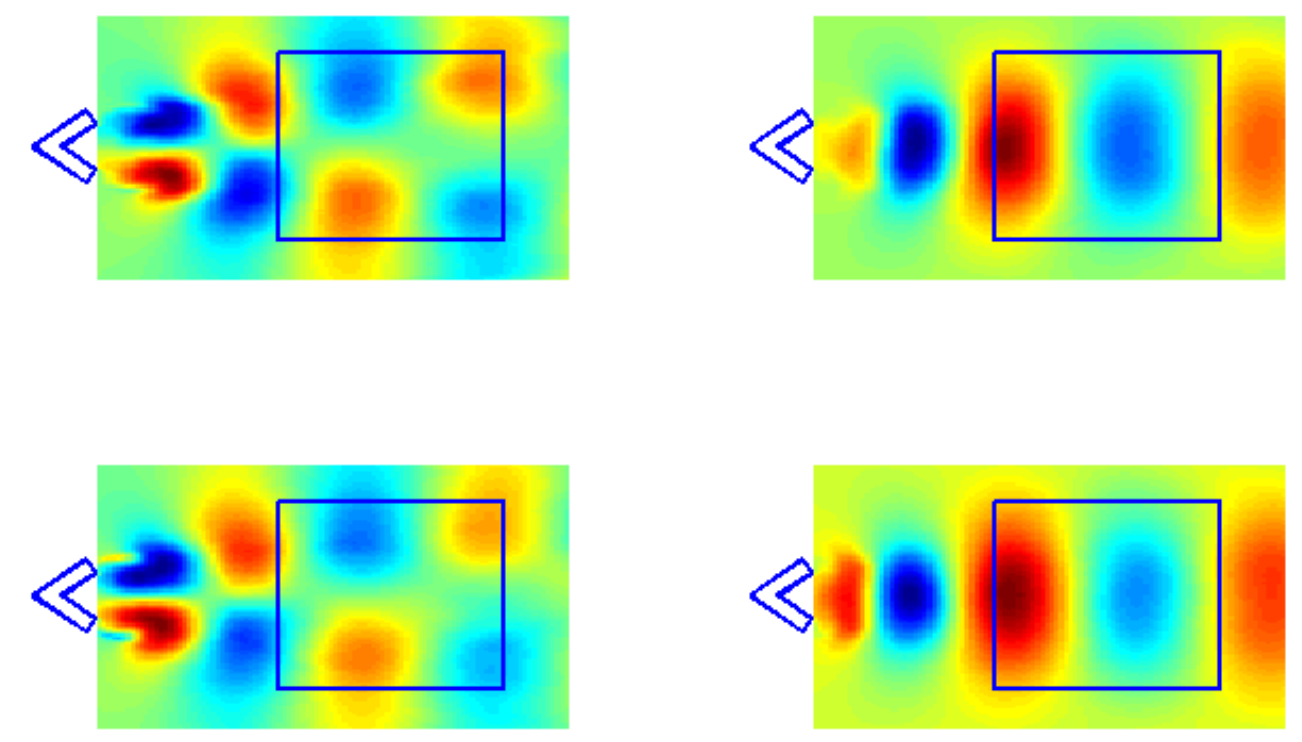

Figure 1.18 - Comparison between the sum of the first three POD modes of the baseline simulation (top) and periodic side "wall" configuration (bottom). Contours of the U-component (streamwise) of velocity are shown on the left and V-component contours are shown on the right. 


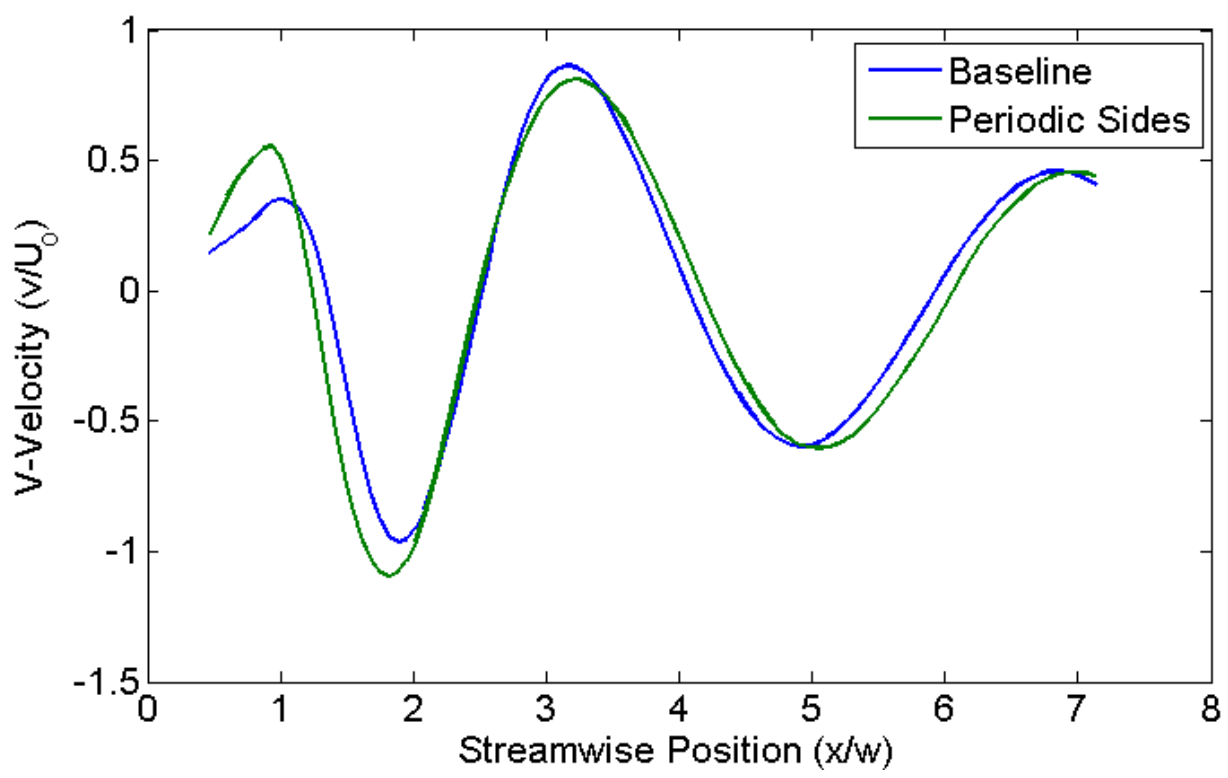

Figure 1.19 - Comparison of the first v-components (vertical) of velocity of the sum of the first three modes between baseline simulation and the configuration with periodic sides.

\subsubsection{Turbulent Inlet}

The fourth study of the series consisted of replacing the uniform velocity of the baseline inlet boundary condition that specifies a spatially and temporally varying velocity field to simulate the effect of upstream turbulence entering the domain. This method, referred to as spectral synthesis, is based on the work of Kraichnan [39] in simulating a divergence free velocity field by summing a series of, in this case 100, Fourier harmonics. The work was adapted by Smirnov [40] to the technique presently implemented in FLUENT which is intended specifically for use in large eddy simulations. For this simulation the turbulence intensity was taken to be 5\% based on an analysis of PIV data taken from a test without the vee-gutter in the test section. The turbulent length scale was taken to be $10 \mathrm{~mm}$ based on the same analysis.

The wake dynamics resulting from this change showed two differences in the shapes of the shedding modes. First, as seen in figure 20, the shapes of the v-velocity modes are skewed such that they are no longer symmetric across the $\mathrm{x}$-axis though they remain approximately the same size and intensity. Second, also shown in figure 1.20, except for the oscillations directly 
behind the vee-gutter, the oscillations in the u-component of velocity show reduced intensity and irregular boundaries compared with the baseline.
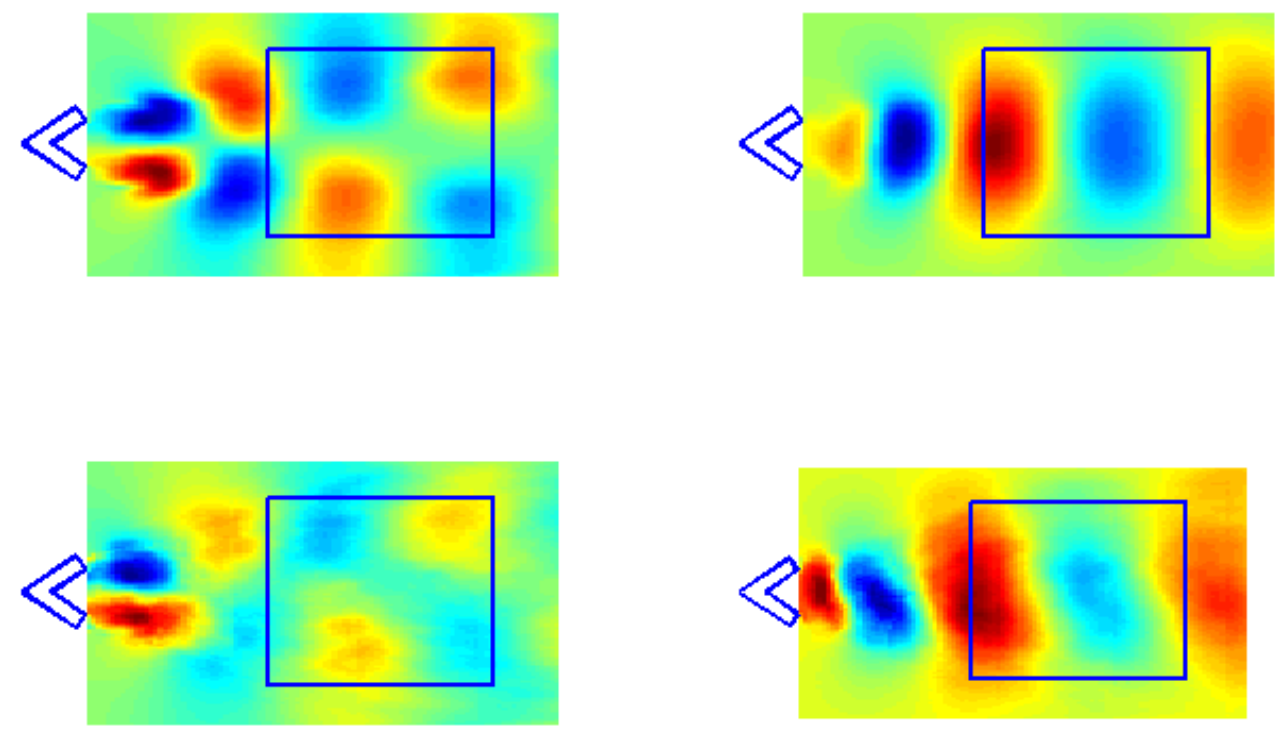

Figure 1.20 - Comparison between the sum of the first three POD modes of the baseline simulation (top) and turbulent inlet configuration (bottom). Contours of the U-component (streamwise) of velocity are shown on the left and V-component contours are shown on the right.

The v-component profile of the sum of the first three modes of the turbulent inlet condition along the $\mathrm{x}$-axis plotted together with the same plot of the baseline condition shows that in spite of the change to the symmetry of the mode shapes shown in figure 20 , the turbulent inlet case matches the baseline profile quite closely (figure 21) for the first $3 \mathrm{w}$ downstream of the vee-gutter, further downstream the only real difference from the baseline is a somewhat lower magnitude of the oscillations. 


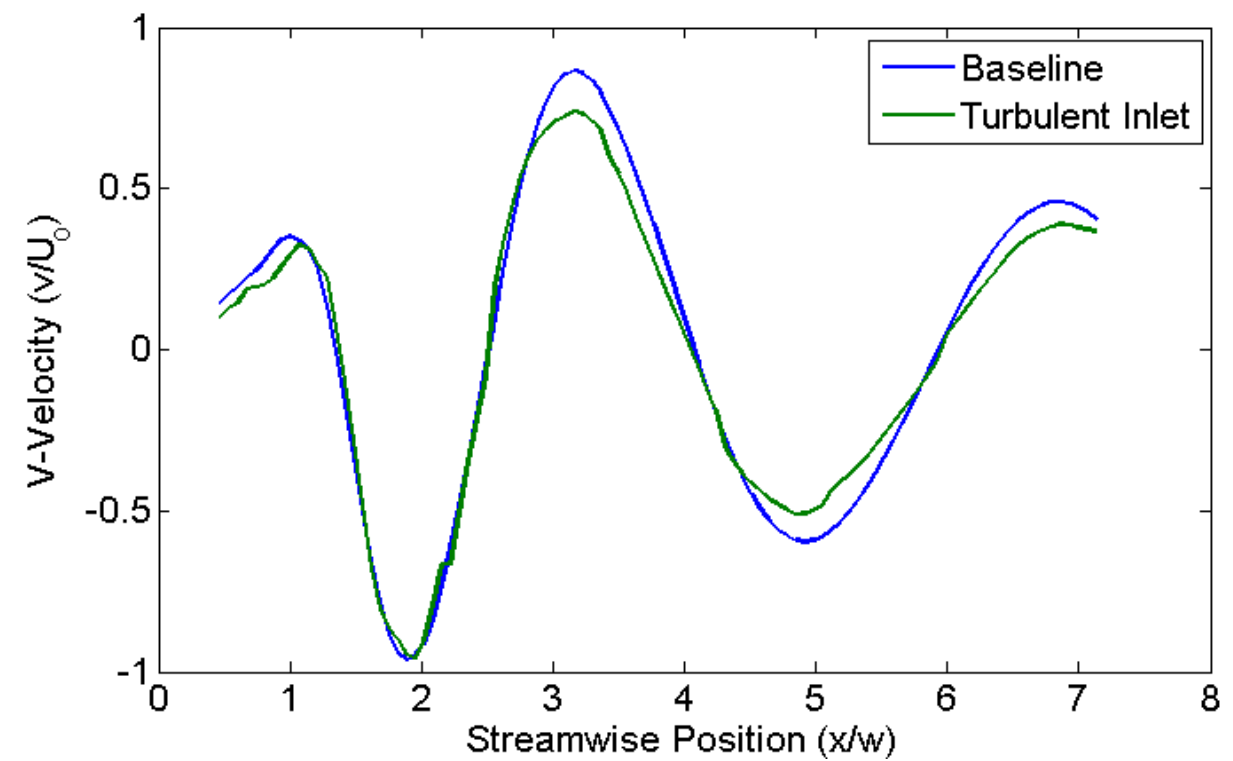

Figure 1.21 - Comparison of the v-components (vertical) of velocity of the sum of the first three modes of the baseline simulation and the configuration with the turbulent inlet.

\subsubsection{Refined Grid}

To study the effect of grid resolution on the dynamics of the simulation, the baseline mesh was refined by doubling the number of cells in each direction, effectively converting each cell from the baseline mesh into 8 equally-sized small cells. The size of the timestep was likewise halved to maintain a constant CFL number. As a result, to simulate a given amount of physical time, the refined grid required a factor of $2^{4}=16$ more computational cycles and $2^{3}=8$ time more memory than the baseline.

The first three modes of the simulation on the fine grid show very similar mode shapes and intensities when compared against the baseline simulation except, as seen in figure 1.22, for the very first oscillation immediately downstream of the vee-gutter. This is more apparent in figure 1.23 which clearly shows that for $\mathrm{x} / \mathrm{w}<2$ the oscillations of the $\mathrm{v}$-component of velocity are nearly double in the refined mesh simulation compared against the baseline. Farther downstream however, the two profiles are much closer to one another. This behavior of the shedding mode seems to be a result of the subgrid scale model delaying and damping out the oscillations of the wake by increasing the effective viscosity in the shear layer for $0<\mathrm{x} / \mathrm{w}<1$. This is most clearly seen in figure 24 which shows a comparison of contours of the Pope criterion 
between the baseline and refined meshes. The kinetic energy of the shear layer is almost entirely modeled by the SGS, indicated by a Pope criterion near unity, in the baseline mesh in the $x / w<1$ region whereas the Pope criterion in the refined grid never exceeds the recommended value of 0.2 in the same region.

This result is interesting in itself but also helps to explain some of the results that were reported in the other studies, namely that the magnitude of the downstream oscillations is relatively insensitive to the magnitude of those farther upstream. That is, the wake oscillations are not simply convected downstream from where they are initially formed but rather decay at a rate that. Thus, even though the baseline simulation is technically under-resolved (according to the Pope criterion) near the vee-gutter, the overdamped oscillations that this produces don't persist into the region where the PIV data was taken. So, in spite of the significant differences in SGS activity, both the baseline and refined-grid simulations show good agreement with the PIV data's shedding mode.
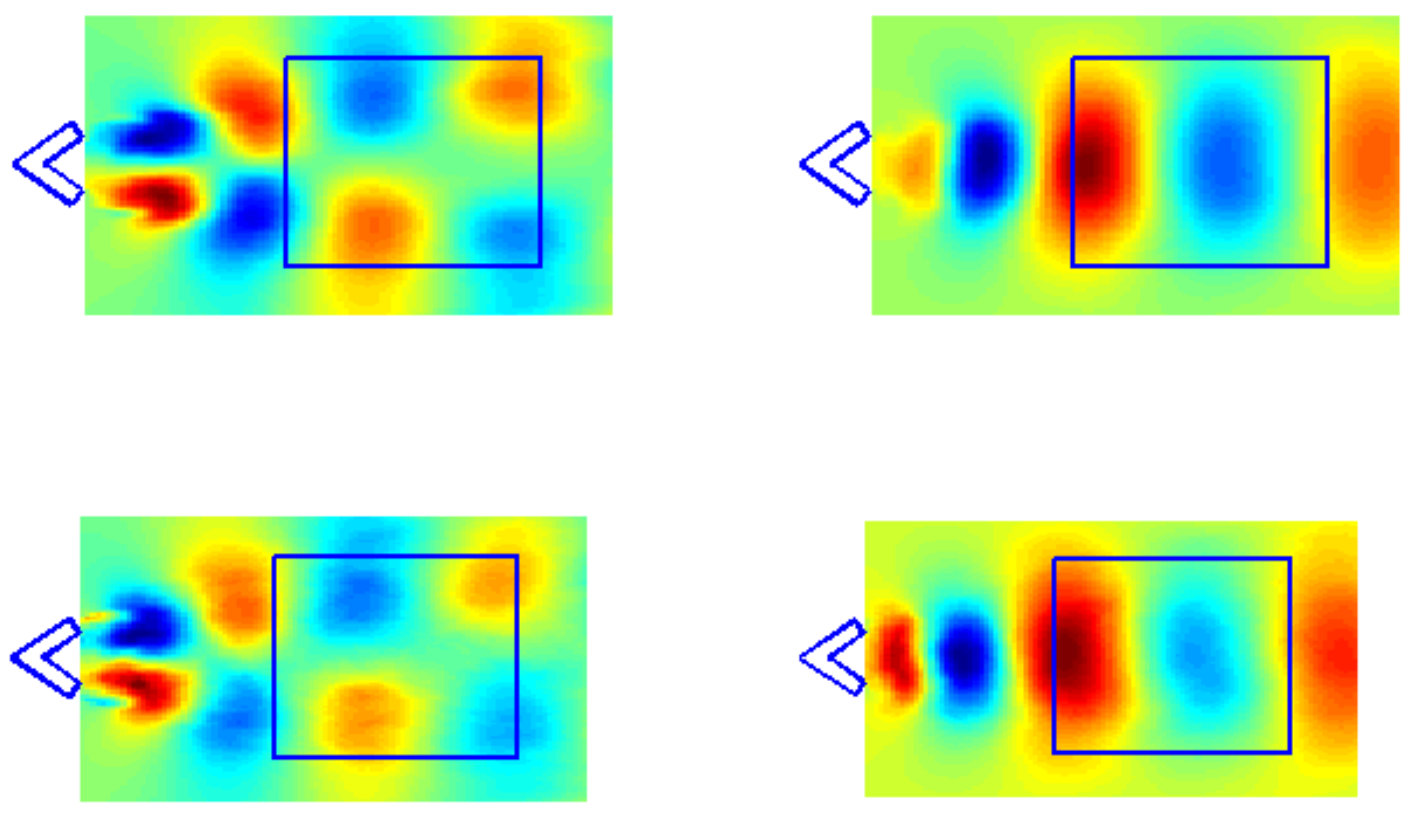

Figure 1.22 - Velocity contours of first three modes of baseline (top) and refined grid (bottom) simulations, showing contours of u' (left) and v' (right). 


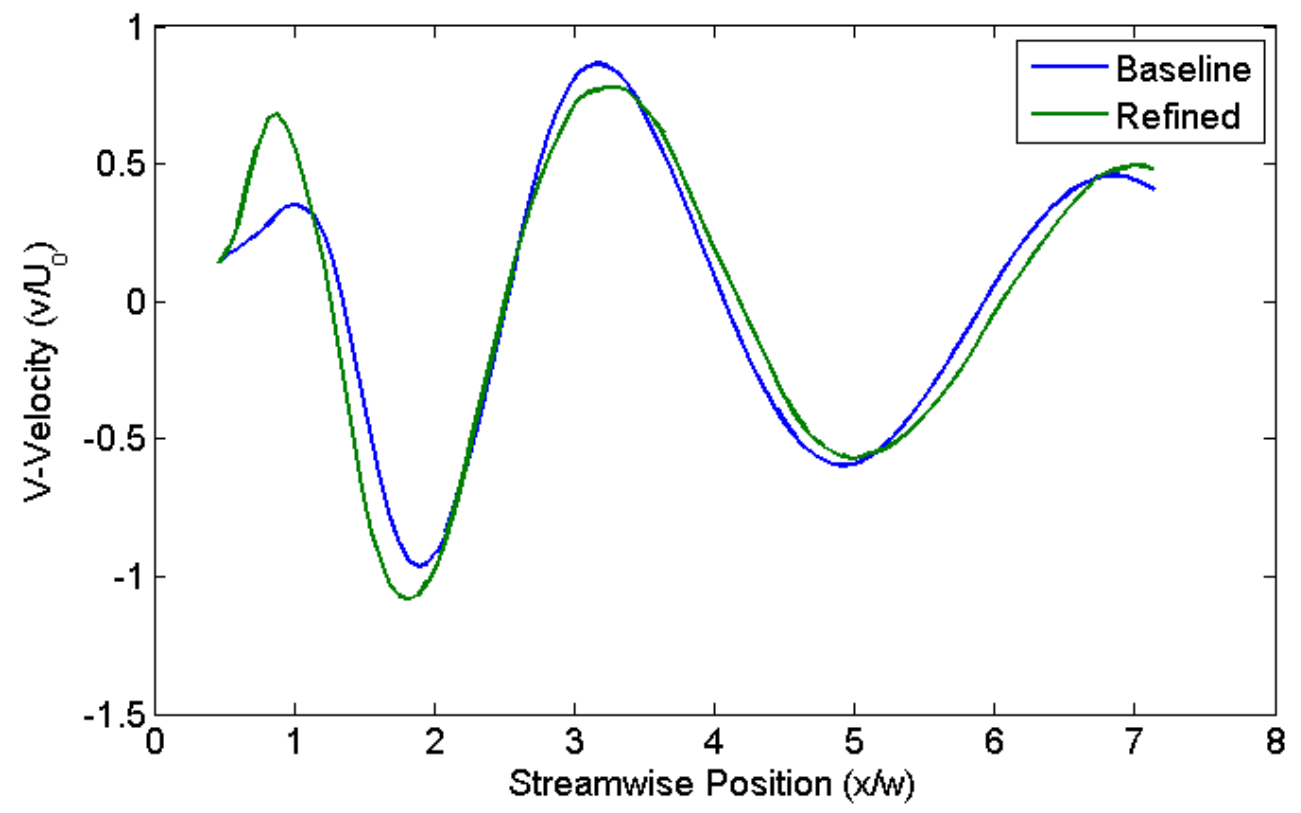

Figure 1.23 - Comparison of the v-components (vertical) of velocity of the sum of the first three modes of the baseline simulation and the refined grid simulation. 

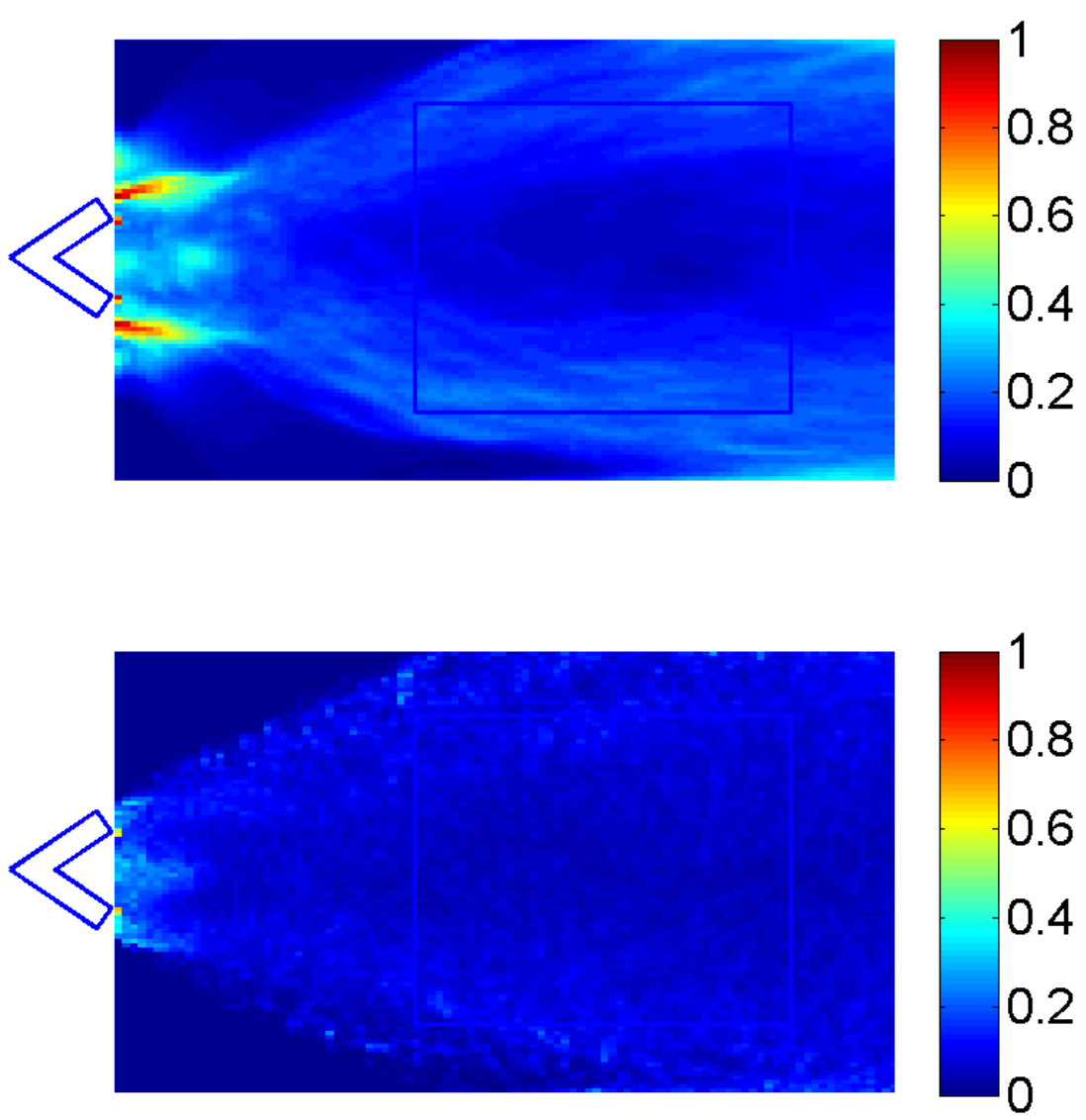

Figure 1.24 - Pope Criterion contours at midplane for baseline (top) and refined mesh (bottom) cases.

\subsubsection{POD Convergence}

Since the proper orthogonal decomposition deals with the manipulation of large matrices the computational costs of performing the decomposition are not trivial. Solving the eigenvalue problem on the correlation matrix in particular requires significant time and memory to complete. For this work, these calculations were performed with the commercial software MATLAB which, for symmetric positive definite matrices like the correlation matrix, solves the eigenvalue problem using Cholesky decomposition, which requires $\mathrm{O}\left(\mathrm{N}^{3}\right)$ operations to complete as shown in figure 1.25. 


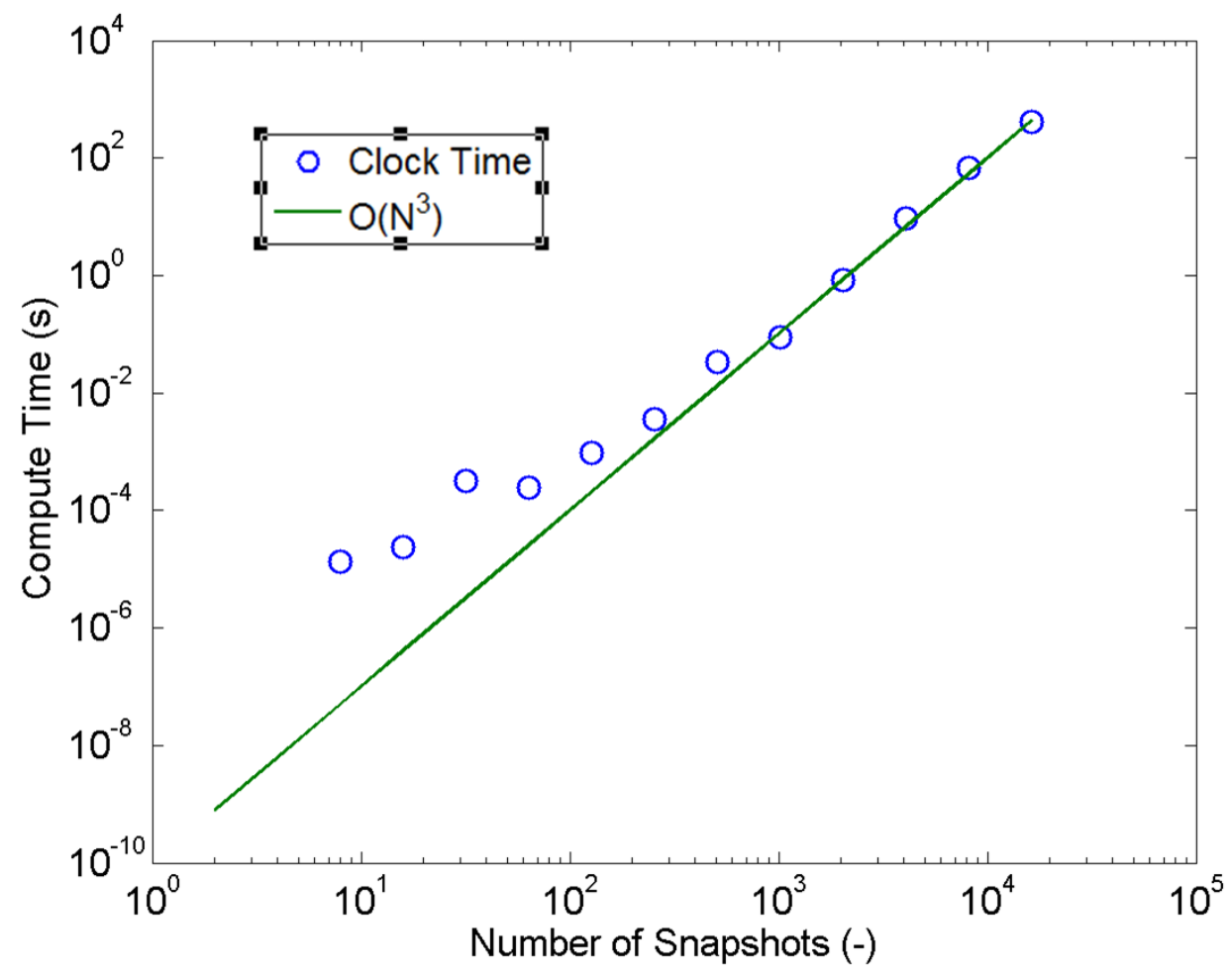

Figure 1.25 - Computation time vs number of snapshots $\mathrm{N}$ showing that the computational time grows proportional to $\mathrm{N}^{3}$ as expected (green line is $\sim N^{3}$ ).

Thus, as the size of data sets increases the computational costs can quickly become prohibitive. In an effort to understand the tradeoffs between accuracy and cost, a study was done to characterize the convergence behavior of eigenvalues and eigenmodes with respect to the number of snapshots used to produce them. For this the POD algorithm was successively applied to larger and larger subgroups of snapshots of a typical dataset starting with 2 snapshots and doubling the number of snapshots used until the entire set of 1024 snapshots was used. The residual of the eigenvalues was defined as the L2 norm of the difference between a given mode produced from different numbers of snapshots. The results show that the eigenvalues themselves shown $2^{\text {nd }}$-order convergence behavior as shown in figure 1.26 but the eigenmodes convergence rate, shown in figure 1.27 , is just $1^{\text {st }}$-order, the same as a typical time-averaging operation. 


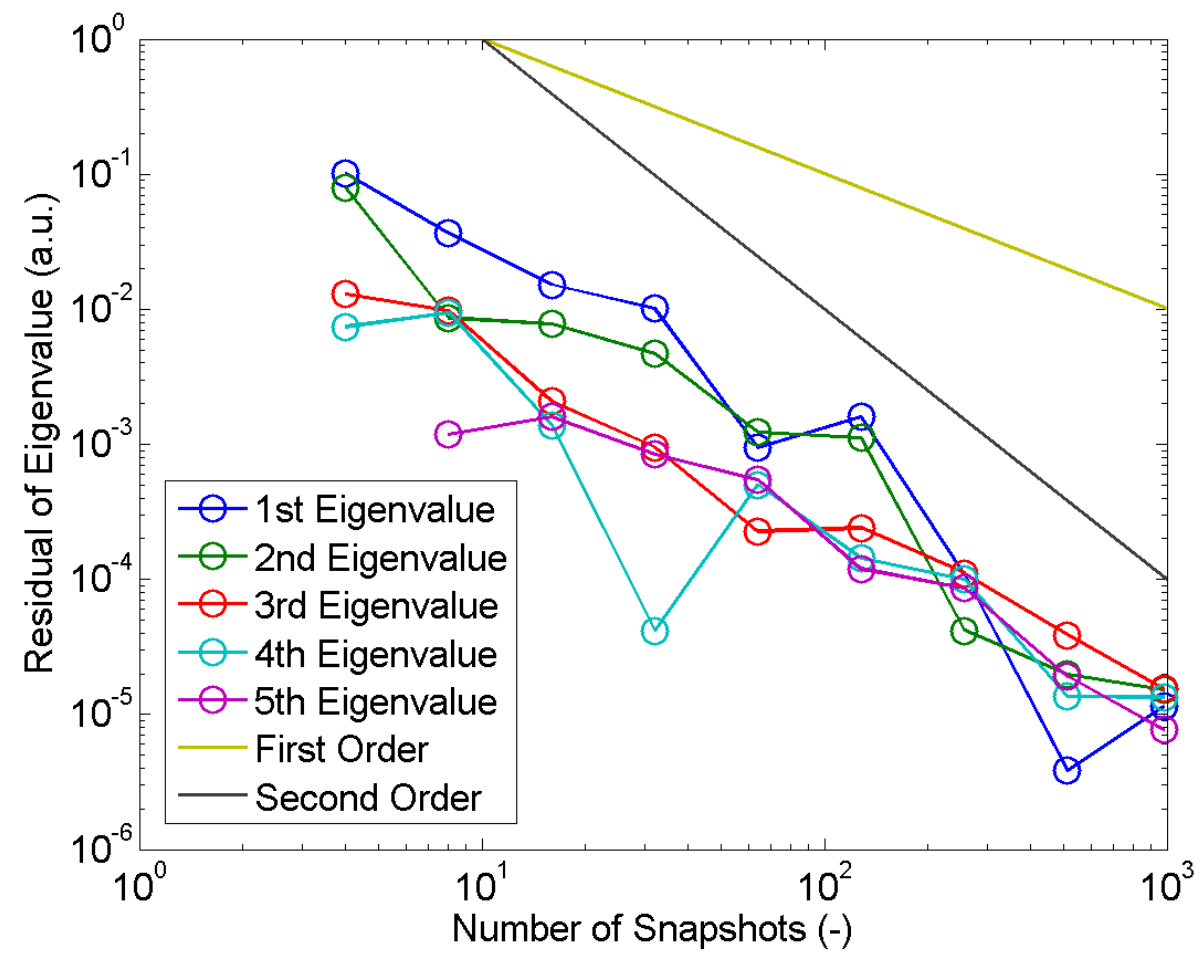

Figure 1.26 - Second order convergence rate of the first five eigenvalues.

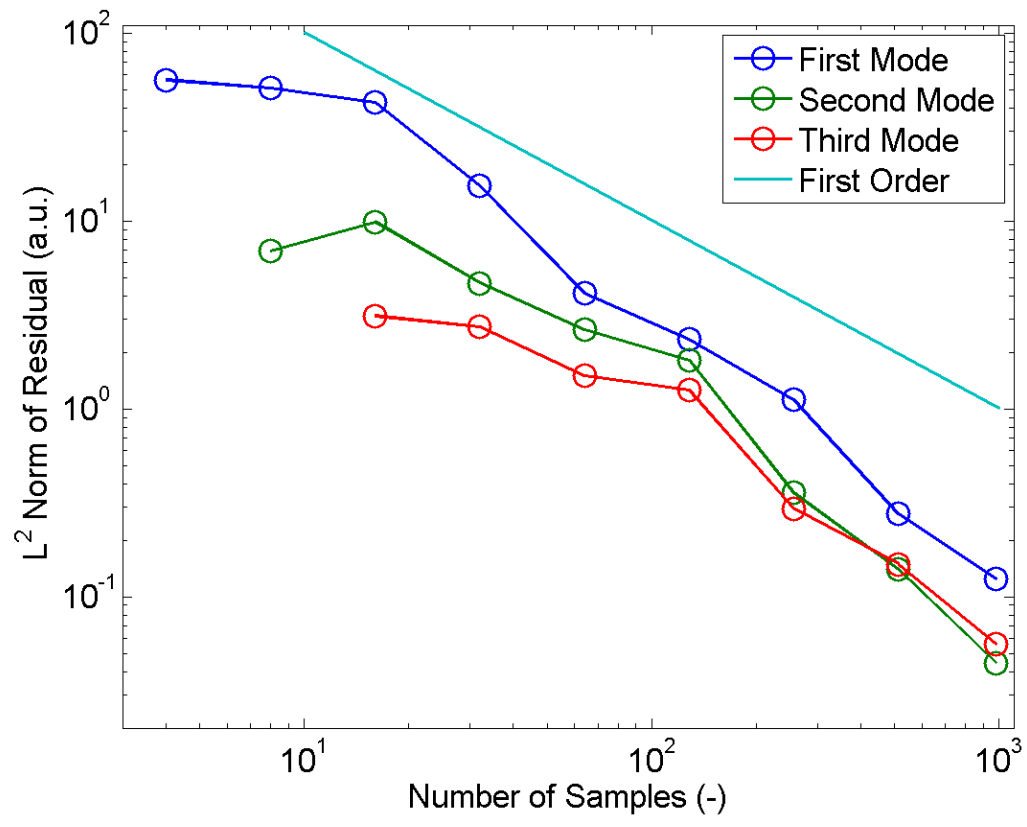

Figure 1.27 - Convergence of First Three POD Modes with Increasing Number of Samples showing a first order convergence rate. 


\subsection{CONCLUSION}

Presented is a systematic study and exposition on the use of POD in quantitative validation of flow results obtained using LES in the non-reacting turbulent wake of a vee-guttertype flameholder undergoing periodic vortex shedding. Both the POD mode shapes and mode magnitudes as measured directly in an experiment have been compared to the LES results for two commonly used solvers, OpenFOAM and Fluent. For the case presented, it is shown that, in addition to the wake mean velocity profiles, the dominant LES flow structures deducted via the first three POD modes are in close agreement with the experimentally obtained results. The sensitivity of the model was examined by varying several parameters including mesh resolution, boundary conditions, and inflow turbulence level and repeating the POD validation procedures. The results indicated an unexpected decoupling between the downstream and upstream amplitudes of oscillations of the wake's shedding mode, indicating that the downstream oscillations are not simply artifacts of the upstream oscillations that have been convected downstream. This decoupling helps explain why the simulations on the coarser grid, which was somewhat under-resolved near the vee-gutter, could still match the PIV data measured farther downstream.

The methodology exhibited in this article provides a framework for the validation of flows with large-scale unsteadiness that dominates the contributions to the Reynolds stresses. It is shown that direct, quantitative comparison of POD modes between experiments and simulation results can be used to provide confidence in simulation results, as well as in assessing the sensitivities of the simulation to inflow parameters. 


\subsection{Nomenclature}

\begin{tabular}{|c|c|}
\hline Str & Strouhal Number \\
\hline$f$ & frequency \\
\hline$w$ & Width of vee-gutter bluff body \\
\hline U & Bulk flow velocity \\
\hline $\operatorname{Re}$ & Reynolds number and similar abbreviations do not use italics \\
\hline$\rho$ & Density \\
\hline$\mu$ & Dynamic viscosity \\
\hline$A$ & Cross-sectional area \\
\hline Ro & Roschko Number \\
\hline $\boldsymbol{u}$ & Snapshot vector of instantaneous data \\
\hline$U$ & Matrix of snapshot vectors \\
\hline C & Correlation Matrix \\
\hline$\lambda$ & Vector of Eigenvalues \\
\hline $\boldsymbol{P}$ & Eigenmode matrix \\
\hline B & Eigenvector matrix \\
\hline$u$ & Velocity, x-direction \\
\hline$v$ & Velocity, y-direction \\
\hline$P$ & Pressure \\
\hline
\end{tabular}


e

D

$T$

$\sigma$

c

V

$\tau$

k

M

Internal energy

Diffusivity

Temperature

Strain rate

Specific heat

Volume

Stress

Kinetic energy

Pope Criterion 


\subsection{References}

[1] Macquisten, M., and Dowling, A., (1993) "Low Frequency Combustion Oscillations in a Model Afterburner." Combustion and Flame, 94 253-264.

[2] Giacomazzi, E., Battaglia, V., and Bruno, C., (2004). "The coupling of turbulence and chemistry in a premixed bluff-body flame as studied by LES." Combustion and Flame, 138 (4), pp 320-335. doi:10.1016/j.combustflame.2004.06.004

[3] Shanbhogue, S. J., Husain, S., and Lieuwen, T., (2009) "Lean blowoff of bluff body stabilized flames: Scaling and dynamics” Progress in Energy and Combustion Science 35 (1) pp 98-120. doi:10.1016/j.pecs.2008.07.003

[4] Cocks, P., Sankaran, V., and Soteriou, M., (2013) "Is LES of Reacting Flows Predictive ? Part 1 : Impact of Numerics" 51 $1^{\text {st }}$ AIAA Aerospace Sciences Meeting 2013, pp 1-29.

[5] Giezendanner, R., Keck, O., Weigand, P., Meier, W., Meier, U., Stricker, W., and Aigner, M., (2003) "Periodic combustion instabilities in a swirl burner studied by phase-locked planar laser-induced fluorescence" Combustion Science and Technology, 175 (4) pp 721-741. doi:10.1080/00102200302390

[6] Roshko, A., (1952) "On the Development of Turbulent Wakes from Vortex Streets" Ph.D. thesis, Mechanical Engineering, California Institute of Technology.

[7] Yang, J., and Tsai, G., (1992) “The Wake Flow Structure of an Open-Slit Vee Gutter" Experimental Thermal and Fluid Science, 5 pp 685-696.

[8] Karniadakis, E., and Triantafyllou, G., (1992) "Three-dimensional dynamics and transition to turbulence in the wake of bluff objects" Journal of Fluid Mechanics, 238 pp 1-30. 
[9] Spalart, P. R., and Squires, K. D., (2004) "The status of detached-eddy simulation for bluff bodies. In The Aerodynamics of Heavy Vehicles: Trucks, Buses, and Trains (pp. 29-45). Springer Berlin Heidelberg.

[10] Shur, M., Spalart, P. R., Squires, K. D., Strelets, M., and Travin, A., (2005). “ThreeDimensionality in Reynolds-Averaged Navier-Stokes Solutions Around Two-Dimensional Geometries" AIAA journal, 43 (6) pp. 1230-1242.

[11] Achenbach, E., and Heinecke, E. (1981). "On vortex shedding from smooth and rough cylinders in the range of Reynolds numbers $6 \times 10^{3}$ to $5 \times 10^{6,}$ Journal of Fluid Mechanics, $109 \mathrm{pp}$. 239-251.

[12] West, G., and Apelt, C., (1982). "The effects of tunnel blockage and aspect ratio on the mean flow past a circular cylinder with Reynolds numbers between $10^{4}$ and $10^{5 \%}$ Journal of Fluid Mechanics, 114 pp. 361-377.

[13] Achenbach, E., (1977) "The effect of surface roughness on heat transfer from a circular cylinder to the cross flow of air" International Journal of Heat and Mass Transfer, 20 pp. 359369.

[14] Pearson, K., (1901). "On lines and Planes of Closest Fit to Systems of Points in Space” Philosophical Magazine, 2 pp. 559-572.

[15] Lumley, J., (1967) “The Structure of Inhomogeneous Turbulent Flows," Atmospheric Turbulence and Radio Wave Propagation, 114 pp. 166-178. 
[16] Gergen, I., and Harmanescu, M. (2012), “Application of principal component analysis in the pollution assessment with heavy metals of vegetable food chain in the old mining areas" Chemistry Central Journal, 6 (1) pp. 156. doi:10.1186/1752-153X-6-156

[17] Smith,T., Moehlis, J., and Holmes, P., (2005) "Low-Dimensional Modelling of Turbulence Using the Proper Orthogonal Decomposition: A Tutorial” Nonlinear Dyn., 41 (1-3) pp. $275-307$.

[18] Sirovich, L., and Kirby, M., (1987) "Low-dimensional procedure for the characterization of human faces," Journal of the Optical Society of America, 4 (3) pp. 519.

[19] Hsieh, T., Chen, J., Chen, L., Chiang, P., and Lee, H., (2011) "Time-course gait analysis of hemiparkinsonian rats following 6-hydroxydopamine lesion" Behavioral Brain Research, 222 (1) pp. 1-9.

[20] Berkooz, Gal, Holmes, P., and Lumley, J., (1993) “The Proper Orthogonal Decomposition in the Analysis of Turbulent Flows" Annual Review of Fluid Mechanics 25 (1) pp. 539-575. doi:10.1146/annurev.fl.25.010193.002543.

[21] Fukunaga, K. (1990). Introduction to statistical pattern recognition. Academic press.

[22] Moin, P., and Moser, R., (2006) "Characteristic-eddy decomposition of turbulence in a channel," Journal of Fluid Mechanics, 200 pp. 471.

[23] Sirovich, L., and Kirby, M., (1987) "Low-dimensional procedure for the characterization of human faces," Journal of the Optical Society of America, 4 (3) pp. 519.

[24] Duwig, C., and Iudiciani, P., (2009) "Extended Proper Orthogonal Decomposition for Analysis of Unsteady Flames," Flow, Turbulence, Combustion, 84 (1) pp. 25-47. 
[25] Böhm, B., Brübach, J., Ertem, C., and Dreizler, A., "Experiments for Combustion-LES Validation," Flow, Turbulence, Combustion, 80 (4) pp. 507-529.

[26] Miranda, A., Le Borgne, Y., and Bontempi, G., (2007) "New Routes from Minimal Approximation Error to Principal Components," Neural Process Letters, 27 (3), pp. 1-14.

[27] Maurel, S., Boree, J., and Lumley, J., (2001) 'Extended Proper Orthogonal

Decomposition: Application to Jet/Vortex Interaction” Flow, Turbulence Combustion, 67 (2) pp. $125-136$.

[28] J. Boree, (2003) "Extended proper orthogonal decomposition: a tool to analyse correlated events in turbulent flows," Exp. Fluids, 35 (2) pp. 188-192.

[29] Iudiciani, P., Duwig, C.,, (2011) "Large Eddy Simulation of the Sensitivity of Vortex Breakdown and Flame Stabilisation to Axial Forcing," Flow, Turbul. Combustion, 86 (3-4) pp. 639-666.

[30] Smagornisky, J., (1963) "General Circulation Experiments with the Primitive Equations," Mon. Weather Rev., 91 (3) pp. 99-164.

[31] Meyers, J., and Sagaut, P., (2006) "On the model coefficients for the standard and the variational multi-scale Smagorinsky model” Journal of Fluid Mechanics, 569 pp. 287.

[32] Germano, M., Piomelli, U., Moin, p., and Cabot, W., (1991) “A dynamic subgrid-scale eddy viscosity model," Physics of Fluids and Fluid Dynamics., 3 (7) pp. 1760.

[33] Lilly, D., (1992) “A proposed modification of the Germano subgrid-scale closure method," Physics of Fluids and Fluid Dynamics., 4 (3) pp. 633.. 
[34] Ranalli, J., (2009) "Spatially Resolved Analysis of Flame Dynamics for the Prediction of Thermoacoustic Combustion Instabilities," Ph.D. thesis, Mechanical Engineering, Virginia Tech.

[35] Chatterjee, P., (2004) “A Computational Fluid Dynamics Investigation of Thermoacoustic Instabilities in Premixed Laminar and Turbulent Combustion Systems," Ph.D. thesis, Mechanical Engineering, Virginia Tech.

[36] Chishty, W., (2005) "Effects of Thermoacoustic Oscillations on Spray Combustion Dynamics with Implications for Lean Direct Injection Systems Effects of Thermoacoustic Oscillations on Spray Combustion Dynamics with Implications for Lean Direct Injection Systems," Ph.D. thesis, Mechanical Engineering, Virginia Tech.

[37] Hendricks, A., (2003) "Determination of Flame Dynamics for Unsteady Combustion Systems using Tunable Diode Laser Absorption Spectroscopy" Ph.D. thesis, Mechanical Engineering, Virginia Tech.

[38] Mattingly, J., (2004) Elements of Gas Turbine Propulsion. New York: McGraw-Hill.

[39] R. Kraichnan, (1970) "Diffusion by a Random Velocity Field," Physics of Fluids, 11 pp. $21-31$.

[40] Smirnov, R., Shi, S., and Celik,I., "Random Flow Generation Technique for Large Eddy Simulations and Particle-Dynamics Modeling," Journal of Fluids Engineering, 123 pp. 359-371. 


\section{Paper \#2 - Simulating Bluff-body Flameholders: On the Use of Proper Orthogonal Decomposition for Combustion Dynamics Validation}

\subsection{ABSTRACT}

Contemporary tools for experimentation and computational modeling of unsteady and reacting flow open new opportunities for engineering insight into dynamic phenomena. In this article, we describe a novel use of proper orthogonal decomposition (POD) for validation of the unsteady heat release of a turbulent premixed flame stabilized by a vee-gutter bluff-body. Large eddy simulations were conducted for the same geometry and flow conditions as examined in an experimental rig with chemiluminescence measurements obtained with a high-speed camera. In addition to comparing the experiment to the simulation using traditional time-averaging and point-wise statistical techniques, the dynamic modes of each are isolated using proper orthogonal decomposition (POD) and then compared mode-by-mode against each other. The results show good overall agreement between the shapes and magnitudes of the first modes of the measured and simulated data. A numerical study of into the effects of various simulation parameters on these heat release modes showed significant effects on the flame's effective angle but also on the size, shape, and symmetry patterns of the flame's dynamic modes.

\subsection{INTRODUCTION}

The unsteady release of sensible heat and flow dilation in combustion processes create pressure fluctuations which, particularly in premixed flames, can couple with the acoustics of the combustion system. This acoustic coupling creates a feedback loop with the heat release that can lead to thermo-acoustic instabilities which can reduce combustion efficiency, increase pollutant formation, or become so severe that they damage the combustor. For vee-gutter-type bluff-body flameholders, the flame is stabilized by recirculating hot combustion products from the veegutter's wake. This makes understanding the flow characteristics of the vee-gutter's wake very important for understanding not just the flameholder's performance in the wet (fueled) mode but in the dry (unfueled) mode as well since many of an afterburner's performance characteristics like ignition, turndown, lean blowout limit, and dry loss feature either substantially reduced reaction rates or no reactions at all.

Numerical simulations of bluff-body flameholders, in either wet or dry operation, are often validated against experimental measurements using time-averages and point-wise statistics 
to evaluate the simulations predictive capabilities; however these techniques fail to capture the combined spatial and temporal nature of the dynamics of these systems. This work uses proper orthogonal decomposition to analyze both experimental and simulated data to isolate and then quantitatively compare the shapes and amplitudes of the dynamic modes of each against one another.

\subsection{MOTIVATION FOR DECOMPOSITION TECHNIQUES}

Significant LES validation efforts against combustion experiments have been reported in the literature, these consist largely of comparisons between measured and simulated timeaverages, Reynolds decompositions, and power spectral density as validation metrics [1 - 3]. While successive moments of the time statistics do represent a natural and intuitively meaningful technique for the validation of most quantities for engineering purposes, and although power spectral density does allow for detailed analysis of the frequency content of a given time series, neither technique can provide an adequate description of the spatio-temporal nature of the system's dynamics. One approach which has been used to attempt to solve this problem is phaselocked sampling, which has been successfully used to study flames with periodic instabilities [4]. This technique however requires an external forcing source which can be used to trigger the measurement which prevents it from being applied to self-excited flames. Instantaneous snapshots are often shown in the literature to illustrate, albeit qualitatively, the spatial arrangement of a dominant flow phenomenon. Such snapshots are clearly valuable for qualitative validation but are insufficient for quantitative comparisons to similar snapshot from a different system and can obscure less-dominant flow features which may be of importance but which are overshadowed by the more dominant structures.

Decomposition techniques, such as proper orthogonal decomposition (POD) allow many of these shortcomings to be overcome and allow for reproducible, statistically converged representations of the key flow features and their various magnitudes, time scales, and spatial distributions. These techniques use instantaneous realizations of the system with sufficient spatial and temporal range and resolution such that the relevant flow structures are sufficiently represented. Ensembles of these instantaneous snapshots can be decomposed in space and/or time to separate the various coherent modes from each other in a way that allows for a statistically representative, reproducible, and quantitative description of each. Because such 
decompositions rely only on the input data, and require no underlying model or approximation, they are said to be agnostic to the source of the data, that is, the decomposition technique makes no distinction between data generated by a physical measurement or a numerical simulation. This makes these techniques well suited to the comparisons of measured and simulated dynamics shown here.

\subsection{REVIEW OF LITERATURE}

This section will give a brief overview of the relevant literature for each of the key topics relevant to this work including: decomposition techniques using proper orthogonal decomposition and dynamic mode decomposition, premixed combustion, bluff-body stabilized premixed flames, and large-eddy simulation of premixed flames.

\subsubsection{Wake Dynamics of Bluff Bodies}

The wakes of bluff bodies in non-reacting flows is a topic that has been thoroughly studied through both experimental [5-6] and numerical means [7-9]. Such wakes typically exhibit an alternating shedding pattern where the bluff body sheds vortices from alternating sides as shown in figure 2.1 .

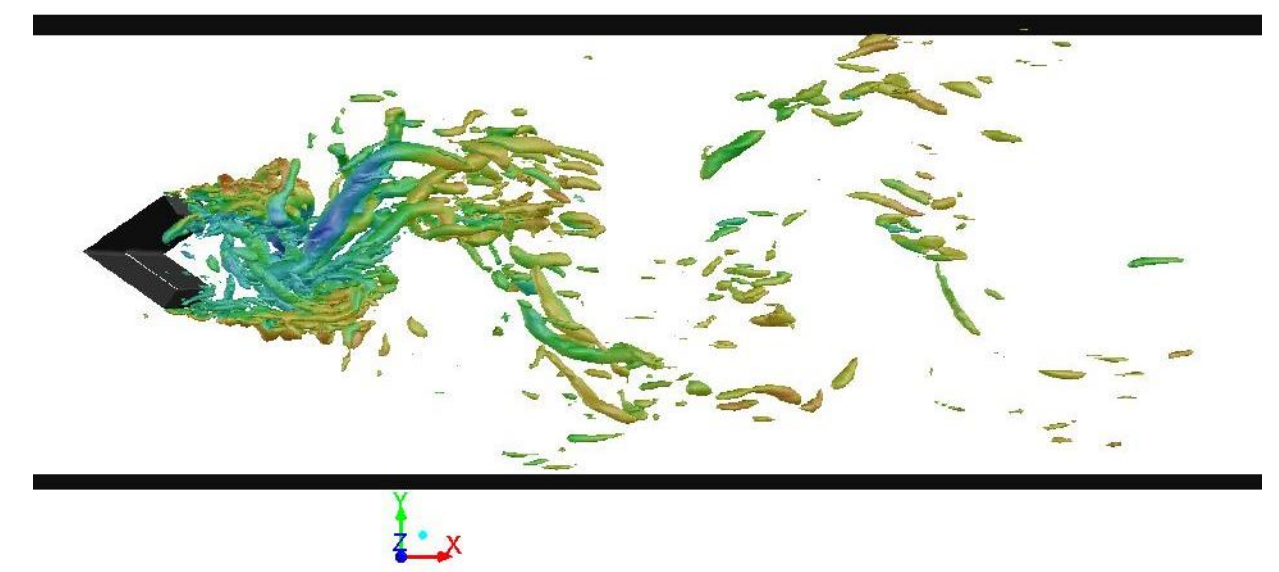

Figure 2.1 - Large eddy simulation from current work of non-reacting flow around a bluff body. The alternating shedding pattern in the bluff-body's wake can be seen from pattern of the vorticity isosurfaces. 
The frequency of this shedding, typically non-dimensionalized as the Strouhal number defined in equation 2.1, has been shown to take values of 0.1-0.5 depending on the geometry of the bluff body, its aspect ratio (ratio of length spanwise direction to a characteristic length scale of its cross-section), the Reynolds number of the flow (equation 2.2), the nature of the boundary layer separation upstream of the wake, and the blockage ratio (fraction of the duct's cross-section blocked by the bluff body, equation 2.3) [10 - 11].

$$
\begin{gathered}
\text { Str }=\frac{f w}{U} \\
\operatorname{Re}=\frac{\rho U w}{\mu} \\
\text { Blockage }=\frac{A_{\text {Bluff Body }}}{A_{\text {Duct }}}
\end{gathered}
$$

The character of the wake's flow conditions can be further characterized by the Roshko Number, $R o$, which is essentially the product of the Reynolds number and the Strouhal number, and describes the turbulence of the wake [5].

$$
R o=\frac{\rho f w^{2}}{\mu}
$$

For cylinders in cross-flow, shedding frequencies have been shown to transition from approximately $\operatorname{Str}=0.21$ at Reynolds numbers below $10^{5}$ and then jump suddenly to approximately $\operatorname{Str}=0.5$ for $10^{5}<\operatorname{Re}<10^{6}$ and then fall back to around $\operatorname{Str}=0.26$ for $\operatorname{Re}>10^{6}$ [12] It was also shown that this intermediate shedding regime could be effectively removed, that is transition directly from the lower Re regime to the higher, by artificially roughening [10]. Other work has shown that the bluff body's size relative to the size of the duct's major and minor cross-section dimensions can affect the frequency of the shedding of the vortices and the distance downstream of the bluff-body that the coherent structures will be formed [13]. 


\subsection{BLUFF BODY STABILIZED FLAMES}

Like the non-reacting case, the oscillating wakes of bluff-body in reacting flows has been the subject of considerable study [1 -2]. These were the dominant means of flame stabilization in turbine engine afterburners for decades [14]. These are ignited by means of a pilot flame or electrical arc in the low-velocity wake of the bluff body where the flame kernel is shielded from the fast-moving bulk flow and allowed to grow. Once lit, bluff body flameholders stabilize the flame by recirculating combustion products from the bluff-body's wake, or ignition zone, into the shear layer where it mixes with the unburned fuel and air mixture causing it to ignite and produce more combustion products, some of which flow back into the recirculation zone and keep the cycle going as illustrated in figure 2.2.

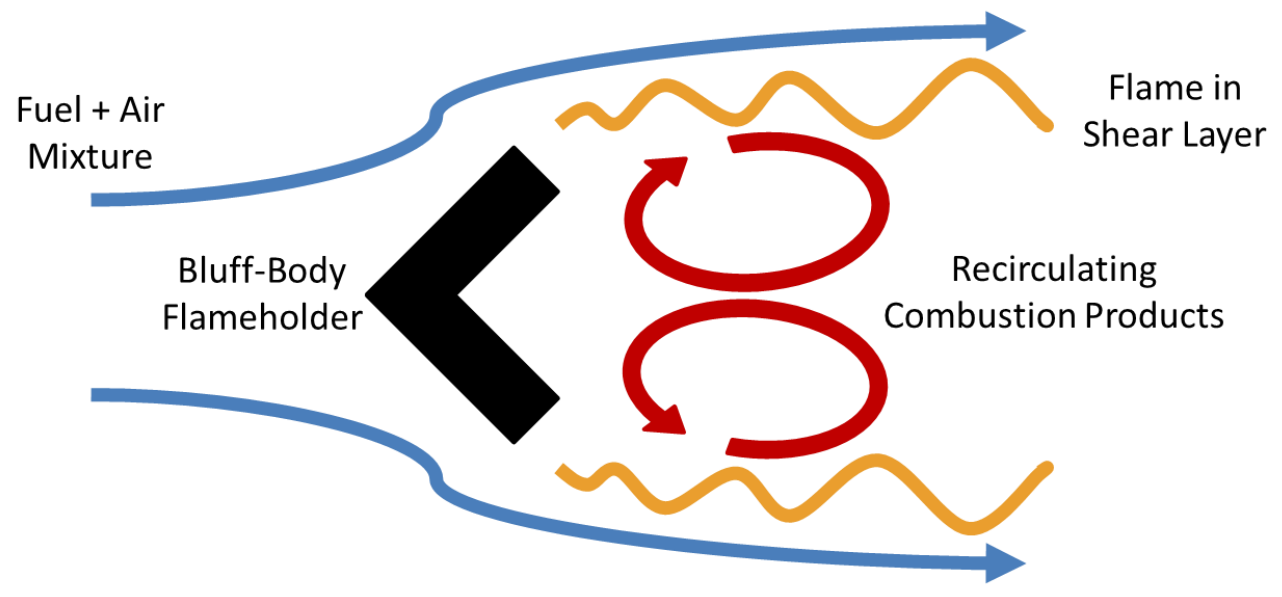

Figure 2.2 - Schematic of a bluff-body stabilized premixed flame showing the recirculation of combustion products from the bluff-body's wake into the shear layer where it mixes and ignites the unburned fuel and air mixture.

The shedding pattern in the bluff body's wake undergoes a drastic change during the transition between non-reacting and reacting modes. A numerical study of flames stabilized by prismatic bluff bodies showed that in the non-reacting case "the flow is characterized by a recirculation zone downstream of the bluff body featuring periodically asymmetric vortex shedding" whereas in the presence of a flame, "the flow is characterized by a recirculation zone 
downstream of the bluff body anchoring the flame and periodically altered by symmetric vortex shedding" [2]. Experimental studies examining the turbulent spectra of similar configurations showed clear evidence of regular shedding in the case of cold flow case whereas when fuel was added and the flow was ignited the spectra was suppressed across all frequencies and actually indicated no preferred shedding mode [3]. These results have been produced in multiple other experimental and numerical studies [15-18].

The frequency of this symmetric shedding has also been the subject of experimental study. One study investigated combustion dynamics of a flame stabilized by an axisymmetric bluff-body by measuring global heat release fluctuations with a photomultiplier tube (PMT). This study showed that the dominant peak in the emission spectra changed in both frequency and amplitude with changes in inlet temperature, vitiation level, inlet Mach number, and equivalence ratio [1]. The range of reported shedding and oscillation frequencies in reacting flow cases seems to be much wider than the non-reacting studies. The cold flow studies previously cited found a relatively small range of Strouhal numbers $(0.16<S t r<0.2)$ even with relatively large changes in geometry and flow conditions, the range of reacting flow cases extended higher and much lower than the limits of the non-reacting range $(0.04<S t r<0.25)$ indicating more complicated physics as well as the potential for oscillations of significantly lower frequency.

This change in shedding behavior has been linked to, among other things, the dilation of the flow due to heat release during combustion. Similar effects, sometimes referred to as vortex breakdown, have been observed in other flow configurations (e.g. swirl-stabilized premixed burners) where coherent vertical structures present in the non-reacting case vanish or are significantly diminished after ignition [19 - 22].

In terms of a mathematical description, the effect of dilation on vorticity can be seen in the vorticity equation for variable density flows is shown as equation 5. The left hand side (LHS) of this equation contains standard terms for the unsteady convection of vorticity, but the right hand side (RHS), particularly its first two terms which are not present in flows with constant density, are significant for understanding the dynamics of such flows. The first term on the RHS represents volumetric expansion due to heat release which effectively acts as a vorticity sink by expanding velocity gradients to a longer length scale as than the original, higher density of its unburned state. This term is very important to the bluff-body problems considered here as the flame exists in a shear layer which would normally exhibit higher vorticity due to the velocity 
deficit in the bluff body wake but which, due to the dramatic density drop for fluid crossing flame and shear layer, is substantially reduced. The second term which governs the baroclinic production of vorticity, is less important for the current study since the pressure gradient and the density gradient are roughly aligned perpendicular along the shear layer, but may be of greater importance for other (e.g. swirling) burners.

$$
\frac{\partial \vec{\omega}}{\partial t}+(\vec{u} \cdot \nabla) \vec{\omega}=-\vec{\omega}(\nabla \cdot \vec{u})+\frac{\nabla \rho \times \nabla p}{\rho^{2}}+(\vec{\omega} \cdot \nabla) \vec{u}
$$

\section{Chemiluminescence}

To capture the spatial and temporal dynamics of the flame, the emissions of the $\mathrm{CH}^{*}$ excited intermediate species were imaged with a high-speed camera. The $\mathrm{CH}^{*}$ chemiluminescence, produced within the flame during oxidation of $\mathrm{C}_{2} \mathrm{H}$ by molecular oxygen [23], emits within a narrow band of wavelengths around 430nm and has been successfully used to quantify unsteady heat release in premixed flames [24 - 25]. These emissions were filtered from the rest of the spectrum with a 430nm +/-10nm bandpass filter before being imaged by a Photron Fastcam SA-4 high speed camera at a framerate of $5 \mathrm{kHz}$. These measurements have been successfully used to extract quantitative frequency information from transient and unsteady combustion processes [26].

\section{Large Eddy Simulation Method}

The method of Large Eddy Simulation of fluid flows has been an active area of research since it was first proposed 50 years ago [27]. The underlying principle remains the same however, namely that the Navier-Stokes equations that govern fluid flow can be split between those scales of motion that can be adequately resolved by the discrete numerical grid used for the simulation, and those that cannot. The variables that are resolved by the grid are referred to as the filtered variables and the effect of the fluid motions too small to be captured directly is modeled in the equations of motion of the filtered variables as shown in the filtered continuity, momentum, and energy equations shown below as equations $2.6-2.9$. 


$$
\begin{gathered}
\frac{\partial \bar{\rho}}{\partial t}+\nabla \cdot(\bar{\rho} \overrightarrow{\vec{u}})=0 \\
\frac{\partial \bar{\rho} \overrightarrow{\vec{u}}}{\partial t}+\nabla \cdot(\bar{\rho} \overrightarrow{\bar{u}} \overrightarrow{\vec{u}})=-\nabla \bar{P}+\nabla \cdot\left(\sigma_{i j}\right) \\
\frac{\partial \bar{\rho} \bar{e}}{\partial t}+(\overrightarrow{\vec{u}} \cdot \nabla) \bar{e}=\nabla \cdot\left(-\bar{\rho} \overrightarrow{\bar{u}} \bar{P}+\bar{\rho} D_{t h} \nabla \bar{e}\right)+\dot{q} \\
\bar{P}=\bar{\rho} R \bar{T} \\
\bar{e}=c_{v} \bar{T}
\end{gathered}
$$

Clearly the success of any LES approach will be dependent on the how the subgrid scale physics is modeled. In this work, the SGS models used in the momentum equations are all adapted from those originally proposed by Smagorinsky, which invokes the Boussinesq hypothesis, namely that the effect of the eddy motion can be modeled as an increased turbulent viscosity which is added to the molecular viscosity of the working fluid. Smagorinsky's SGS model, given in equation 10, models this viscosity as proportional to the magnitude of the local resolved strain rate, $\dot{\sigma}$, as calculated from then filtered velocity field, and proportional to the square of the filter width, $\Delta$, which is calculated as the cube-root of the volume of the computational shell as shown in equation 2.11. From this model the shear stress tensor can be calculated, as in equation 2.12, demonstrating that the turbulence model, at least for the momentum equation is now closed. The value of the subgrid grid viscosity coefficient, $\mathrm{C}_{\mathrm{S}}$ in equation 2.10, is calculated based on estimates of the local unresolved stresses [28 - 29].

$$
\begin{gathered}
\mu_{\Delta}=\bar{\rho}\left(C_{s} \Delta\right)^{2}|\dot{\sigma}| \\
\Delta=\sqrt[3]{V_{\text {Cell }}} \\
\bar{\tau}_{i j}=\mu_{\Delta}\left[\frac{\partial \bar{u}_{i}}{\partial x_{j}}+\frac{\partial \bar{u}_{j}}{\partial x_{i}}-\frac{2}{3} \frac{\partial \bar{u}_{k}}{\partial x_{k}} \delta_{i j}\right]
\end{gathered}
$$

\section{Large Eddy Simulation of Turbulent Premixed Combustion}

To account for the behavior of the turbulent premixed flame within the LES framework, a progress variable approach is used. This method is well-suited to the work here as it attempts to 
collapse the combined effects of chemistry into a progress variable, $c$, which regards the state of a fluid particle as number between 0 and 1 which corresponds to 'unburned' and 'burned' conditions respectively. This progress variable propagates from the burned gas as governed by equation 2.13 where $\mathrm{S}_{\mathrm{T}}$ is the turbulent flame speed which requires additional closure terms.

$$
\frac{\partial \rho c}{\partial t}+(\vec{u} \cdot \nabla) \rho c=\nabla \cdot\left(\rho D_{t h} \nabla c\right)+S_{T}|\nabla c|
$$

The phenomenological processes that premixed flames exhibit under different flow conditions present a number of problems when attempting to model them numerically since an accurate model must consider the effects of compressibility, turbulence, chemistry, heat release, and the several interactions between them. The problem becomes yet more complicated by the numerical grid used to discretize the flow domain, the individual computational cells of which are often larger in size than the thickness of the flame. To overcome this problem, a thickenedflame approach has been successfully used to represents the flame's processes as a filtered, subgrid scale (SGS) phenomena [30 - 32]. With this treatment the flame is thickened to the length scale of the local grid resolution regardless of the disparity between the two length scales which in turn changes the effective Damkohler and Karlovitz numbers, which can change the dynamic character of the flame structure [33].

The turbulent flame speed closure used in this work is the model by Zimont [34 - 35] to account for the interaction between the flame's resolved and unresolved chemical and turbulent timescales. Shown equations 2.14 and 2.15, this model takes its estimates for the unresolved scales of turbulence directly from the subgrid scale turbulence model, in this case dynamic Smagorinsky. The length- and timescales of the flame's laminar chemistry based on the thermal diffusivity of the unburned mixture, $\alpha$, and the laminar flamespeed, $S_{l}$, which is taken as a model input from experimental measurements which, due to the complexity of detailed chemistry in hydrocarbon flames, is more reliable simulate flamespeeds [36]. 


$$
\begin{gathered}
S_{T}=B u^{\prime-3 / 4} S_{l}^{1 / 2}\left(\frac{C_{S} \Delta}{\alpha}\right)^{1 / 4} \\
u^{\prime}=C_{S} \Delta \sqrt{S_{i j} S_{i j}}
\end{gathered}
$$

\subsubsection{Proper Orthogonal Decomposition}

Proper orthogonal decomposition (POD), is a data analysis technique originally proposed in 1901 [37] and first recognized by Lumley [38] for its value in analyzing turbulent flows. The statistical technique has been applied to data in many different fields that has been applied to a wide range of problems from pollutant dispersion [39] to reduced-order modeling [40] to machine vision [41] to neurology [42]. Owing in part to the diversity of its applications, POD is also referred to by a number of names including: principal component analysis, the KarhunenLoève transformation, the method of empirical orthogonal functions, singular-value decomposition, eigenvalue decomposition, factor analysis, the Eckart-Young theorem, empirical component analysis, the Hotelling transform, among others. The method is essentially a pattern

recognition technique that seeks to approximate a dataset through a linear combination of a minimum number of orthogonal vectors [43].

While limited in aspects in its ability to discriminate between data classes [44], POD's proven application in detecting coherent structures in turbulent [45 - 46] and reacting flows [47 -48], together with its agnosticism toward the source of data, makes this technique particularly well-suited to the comparison of simulated and measured combustion dynamic data.

The method for calculating the POD begins by compiling a "snapshot' of sample data, either measured or simulated, into column vector $\mathbf{u}^{\mathbf{i}}$ in equation 16 , these vectors are then assembled into an $\mathrm{MxN}$ matrix $\mathbf{U}$ in equation 2.17 where $\mathrm{M}$ is the number of samples in each snapshot and $\mathrm{N}$ is the number of snapshots . 


$$
\begin{aligned}
& u^{i}=\left[\begin{array}{c}
u_{1}^{i} \\
u_{2}^{i} \\
u_{3}^{i} \\
\vdots \\
u_{m}^{i}
\end{array}\right] \\
& \boldsymbol{U}=\left[\begin{array}{lllll}
\boldsymbol{u}^{1} & \boldsymbol{u}^{2} & \boldsymbol{u}^{3} & \cdots & \boldsymbol{u}^{N}
\end{array}\right]
\end{aligned}
$$

Each element of the matrix $\boldsymbol{U}$ is then subtracted by the mean of its row [49]. The correlation matrix $\widetilde{\boldsymbol{C}}$ is calculated by premultiplying $\boldsymbol{U}$ by its transpose and dividing by N. Next the eigenvalue problem of $\widetilde{\boldsymbol{C}}$ is solved to find the vector of eigenvalues, $\lambda$, and the matrix of eigenvectors, $\boldsymbol{B} . \quad \boldsymbol{U}$ and $\boldsymbol{B}$ are then multiplied to produce the matrix of unweighted eigenmodes $\boldsymbol{P}$. The properly weighted eigenmodes are calculated by multiplying each unweighted eigenmode with its corresponding eigenvalue.

Where POD is often used to construct a reduced-order model of a system by summing the most energetic modes, there are essentially two applications here, both of which treat each mode (or pair of modes in the case of travelling waves which form sin/cosine pairs) as an isolated physical phenomenon which represents a coherent structure in the data. First, each structure can be used as a means of system identification to qualitatively characterize the system. Second, the properly weighted modes can form the basis for quantitative (in both magnitude and spatial distribution) comparison between similar phenomena isolated from decompositions of different datasets to determine to what extent the two systems share a mode of given dynamic behavior.

\subsection{EXPERIMENT}

This section describes the facilities used for conducting the experimental portion of this work including the laboratory infrastructure, the analyses that were completed as part of the design of the rig, a summary of the design features that enabled the measurement techniques described later, and several characterizations of the conditions in the test section.

\subsubsection{CSDL Facilities}

The experiments described here took place in a newly-constructed test rig that was built for evaluating augmentor flameholder concepts and developing diagnostic techniques to measure 
the physical phenomena of interest in augmentor performance. The new augmentor development rig was built as part of the modular combustion rig housed in the Combustion Systems Dynamics Lab (CSDL) at Virginia Tech which has a background of experimental work in combustion dynamics [23], laminar and turbulent premixed combustion [50], multiphase flow [51] and optical diagnostics (Hendricks 2003) [52]. The lab features a Kaeser FS440 air compressor capable of delivering up to $0.7 \mathrm{~kg} / \mathrm{s}(1.5 \mathrm{lbm} / \mathrm{s})$ of air at up to $10 \mathrm{~atm}(150 \mathrm{psia})$. The lab is also equipped with electrical heaters which preheat air with unvitiated air at temperatures up to $650 \mathrm{~K}$ (700 F). After heat loss and compressibility effects, the envelope of possible inlet temperatures and bulk-flow Mach numbers is shown in figure 2.3.

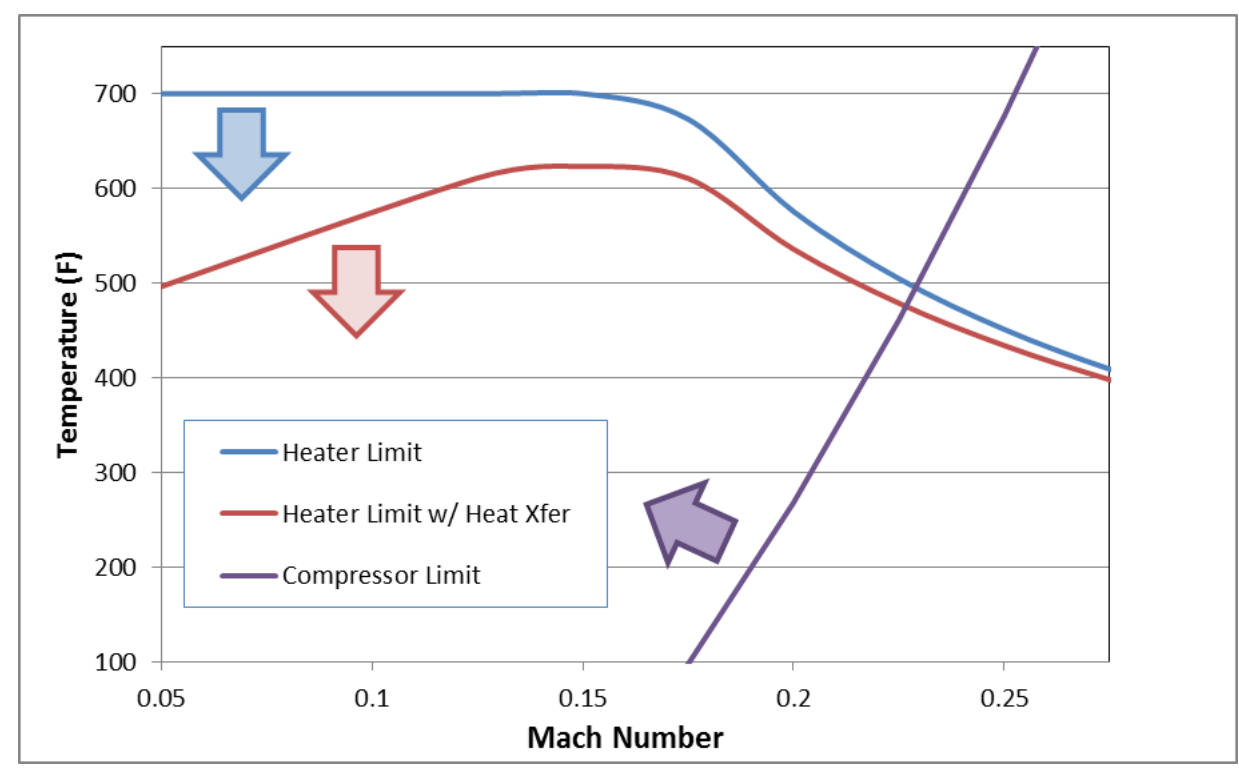

Figure 2.3 - Envelope of section inlet temperature and Mach number limitations from facility constraints.

\subsubsection{Rig Layout and Design}

The augmentor development rig was built as part of the modular combustion rig at the CSDL shown in figure 2.4, consists of three primary components: a fuel-injection section, a converging section, and the test section. 


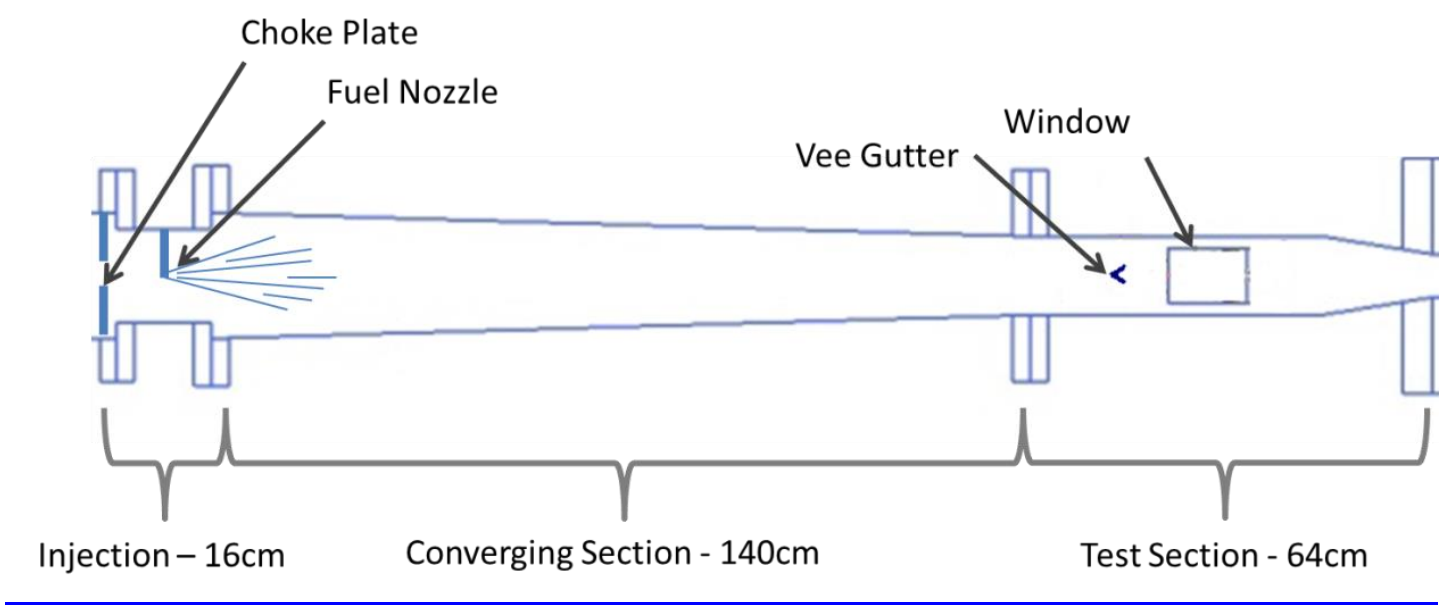

Figure 2.4 - Schematic of experiment showing the three sections of the rig and the locations of the fuel injection, vee-gutter flameholder, and windows.

The test-section features a $12.7 \times 5 \mathrm{~cm}(2 " \times 5 ")$ rectangular cross-section, a $70^{\circ} 3 \mathrm{~cm}\left(1.2^{\prime \prime}\right)$ wide vee-gutter flameholder which horizontally spans the minor axis of the cross-section, and multiple windows for optical access to the flame.

Upstream of the test-section is a converging section 140cm (55") in length that transitions the from a circular, $20 \mathrm{~cm}\left(8^{\prime \prime}\right)$ diameter cross-section to the rectangular cross-section of the rig's test-section. Immediately upstream of the converging section is the injection section where fuel and seed particles are introduced to the heated air. Immediately upstream of the injection section is a choke plate which acoustically isolates the experiment from the rest of the air supply. The liquid fuel delivery systems is capable of supplying $7.5 \mathrm{lpm}(2 \mathrm{gpm})$ of fuel to a pair of spray nozzles that atomize the fuel and ensure proper mixing with the air.

While the test rig was designed to accommodate multiple flameholder configurations including bluff-body and jet-stabilized flameholders, the work presented here focuses solely on a sharp-nosed vee-gutter type bluff-body flameholder. The vee-gutter has a constant, vee-shaped cross-section and is oriented to span the $50.8 \mathrm{~mm}$ ( $2.0 \mathrm{in}$ ) minor dimension of the test section (that is it spans the flow horizontally and perpendicular to the flow). To achieve the desired blockage ratio of 0.24 the vee-gutter was designed to have a tip-to-tip distance of $30.5 \mathrm{~mm}(1.2$ in) and an included 70 degrees so as to be representative of typical afterburner configurations [14]. 
The test-section features three windows, a pair of side windows that are $9 \mathrm{x} 13 \mathrm{~cm}$ (3.5" $\times 5$ ") in size and a single top window $2.5 \times 13 \mathrm{~cm}(1$ " $\times 5$ ") in size and allow for the use of optical diagnostic measurements of the flame. The leading edges of these windows, as shown in figure 2.5, are located $6 \mathrm{~cm}(2.4$ ") downstream of the trailing edge of the vee gutter flameholder.

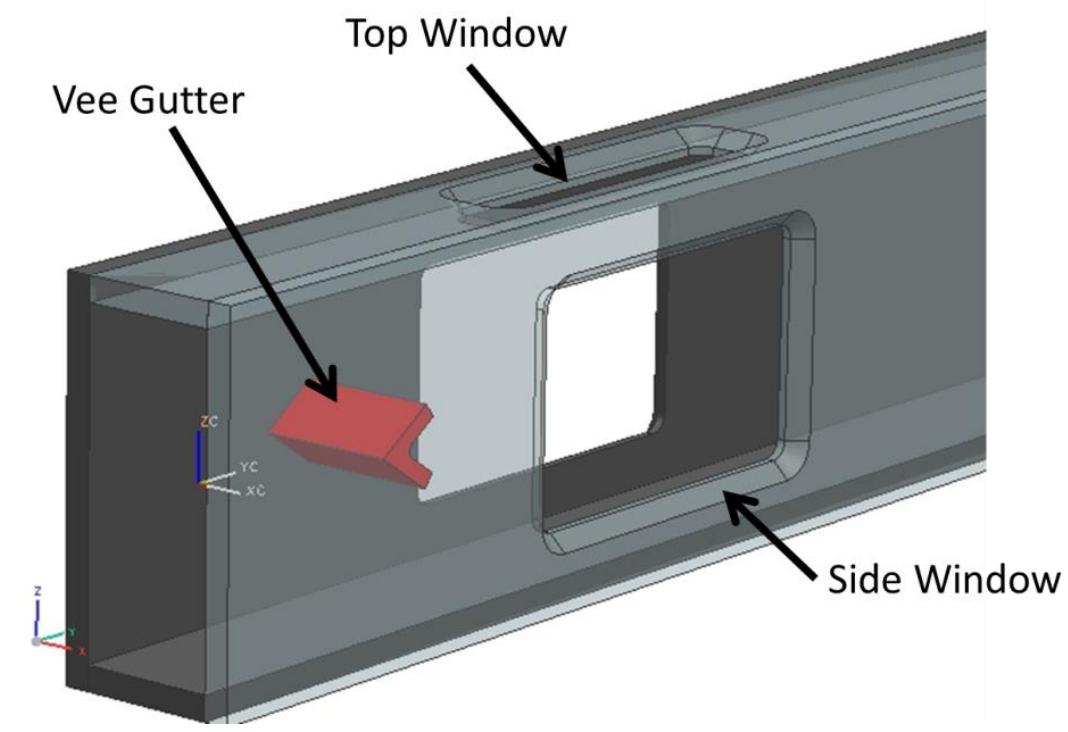

Figure 2.5 - Transparent view of CAD model of the test section showing the two side windows and top window relative to the location of the vee-gutter flameholder.

\subsection{LARGE EDDY SIMULATION}

The simulations reported here were completed using ANSYS Fluent and are based on a single set of operating conditions, the boundary conditions for which are shown in Table 2.1, which corresponds to an in inlet Mach number of approximately 0.12. Additional model input parameters include the laminar flame speed, which was held constant at $0.65 \mathrm{~m} / \mathrm{s}$ based on the work of Vukadinovic [53] and the flame's critical strain which was set to $10^{5} \mathrm{~s}^{-1}$ based on the laminar flame speed and thermal diffusivity of the fluid [54].

Table 2.1 - Boundary conditions for the simulations reported

\begin{tabular}{|c|c|c|}
\hline Parameter & Value & Units \\
\hline $\begin{array}{c}\text { Inlet Mass Flow } \\
\text { rate }\end{array}$ & 0.396 & $\mathrm{Kg} / \mathrm{s}$ \\
\hline
\end{tabular}




\begin{tabular}{|c|c|c|}
\hline Inlet Temperature & 493 & $\mathrm{~K}$ \\
\hline Outlet Pressure & 131 & $\mathrm{kPa}$ \\
\hline $\begin{array}{l}\text { Equivalence } \\
\text { Ratio }\end{array}$ & 1.0 & - \\
\hline
\end{tabular}

The computational domain used for all of the simulations shown here consisted of a constant rectangular cross-section duct a prismatic $70^{\circ}$ degree included angle vee-gutter, with a $3.05 \mathrm{~cm}$ tip to tip measurement ' $w$ ', extending through the minor axis of the duct. The vee-gutter has a thickness of $0.21 w$. As shown in figure 2.6, the domain extends $9.1 w$ upstream of the vee gutters leading edge which is actually longer than the test section itself however this length was chosen based on boundary layer analysis to account for the boundary layer in the transition section that exists prior to the flow entering the test section. The domain is $24.1 \mathrm{w}$ long overall, as shown in figure 2.7 , and maintains a rectangular $1.67 w \times 4.17 w$ cross-section throughout. The real experiment also has a constant cross-sectional area, but begins to transition to a hexagonal cross-section at the end of the water-jacket section, this geometrical difference was assumed negligible.

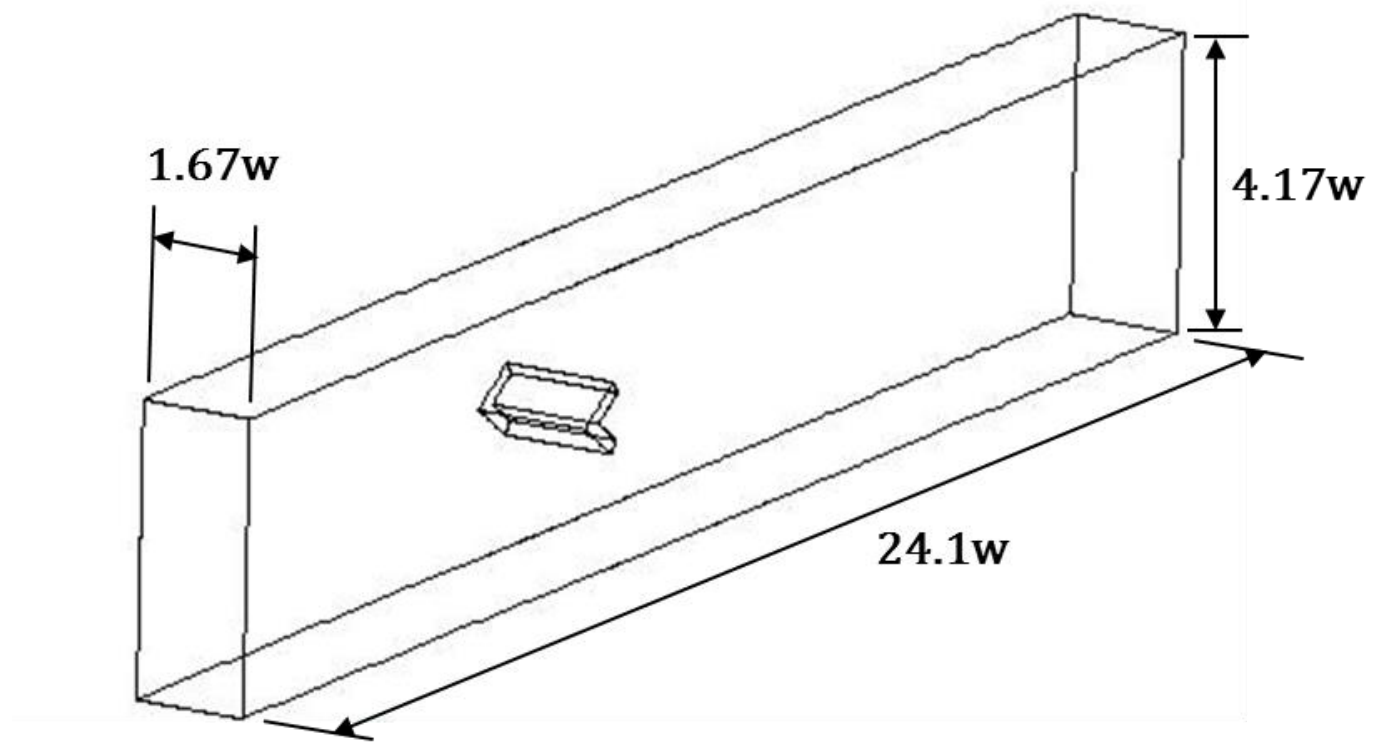

Figure 2.6 - Isometric view of dimensions of the domain used in the LES models based on the width of the flameholder $\mathrm{w}=3.05 \mathrm{~cm}$ and an included angle of $70^{\circ}$. 


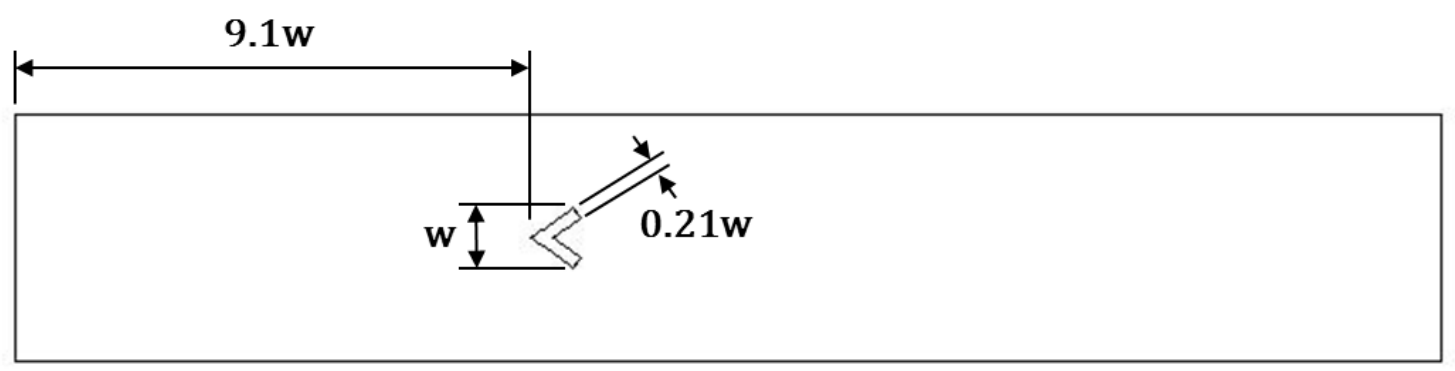

Figure 2.7 - Plan view dimensions of the domain used in LES models.

The mesh used in these simulations consisted of $2.8 \mathrm{M}$ cells, and built in a blockstructured format using ICEM Hexa and then converted to the unstructured formats used by the ANSYS Fluent solver. In making the mesh, care was taken to limit the growth rate in any direction to 1.05 or smaller and the maximum aspect ratio of any cell in the region of interest was limited to 5 or less. Downstream of the region of interest, the aspect ratios of the cells were intentionally stretched in the streamwise direction to dampen out fluctuations in the flow before they reached the outlet boundary.

\subsubsection{Comparison with Experiment}

Since the chemiluminescence signal is a line of sight measurement by nature, each pixel of the high-speed camera's sensor represents an integral quantity of chemiluminescence along its optical path. To account for this in the simulations, the cumulative heat release along simulated lines of sight were calculated. This was done in ANSYS Fluent by using a user-defined-scalar (UDS) which allows for the solution of a generic convection-diffusion partial differential equation for a generic scalar, $\varphi$, represented in equation 2.18 [54].

$$
\frac{\partial \varphi}{\partial t}+\frac{\partial \varphi}{\partial x_{i}}\left[F_{i} \varphi-D \frac{\partial \varphi}{\partial x_{i}}\right]=S_{\varphi}
$$


This equation was modified by setting the diffusion and unsteady terms to zero and then, by means of a set of user-defined-functions (UDFs), manipulated into the form shown in equation 2.19 which equates the growth rate of the passive scalar in the direction of the view vector to the local heat-release rate of the premixed combustion model.

$$
\frac{\partial \varphi}{\partial \vec{x}_{\text {view }}}=\dot{q}
$$

By assuming that the simulated camera is far away the view vectors become parallel everywhere in the domain thus simplifying the analysis. In this case, the view vector simply points in the $\mathrm{z}$-direction, i.e. the spanwise direction. At the side wall farthest from the "camera" the scalar is assigned a Dirichlet-type boundary condition equal to zero. Finally, the side wall nearest the camera's location is treated as the projection plane for the accumulated signal and is assigned a zero-slope Neumann-type boundary condition as shown in the exploded view in figure 2.8 .

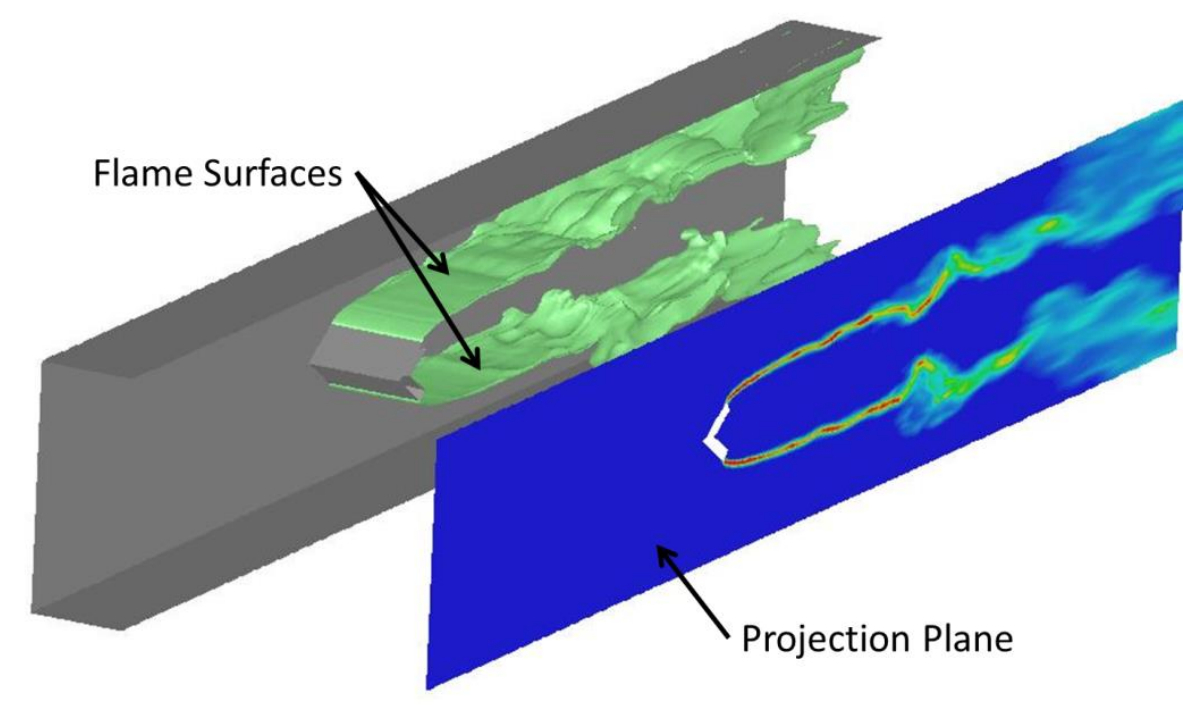

Figure 2.8 - Illustration of flame's heat release imaged to simulate the line-of-sight integrated chemiluminescence imaged in the experiment. 
This signal was then sampled on a 100x100 grid of sampling points on the imaging wall at the locations shown in figure 2.9. For further investigation the heat release rate was sampled on a second set of sampling points with identical $\mathrm{x}$ - and $\mathrm{y}$-coordinates but located at the midplane between the two side walls of the test-section.

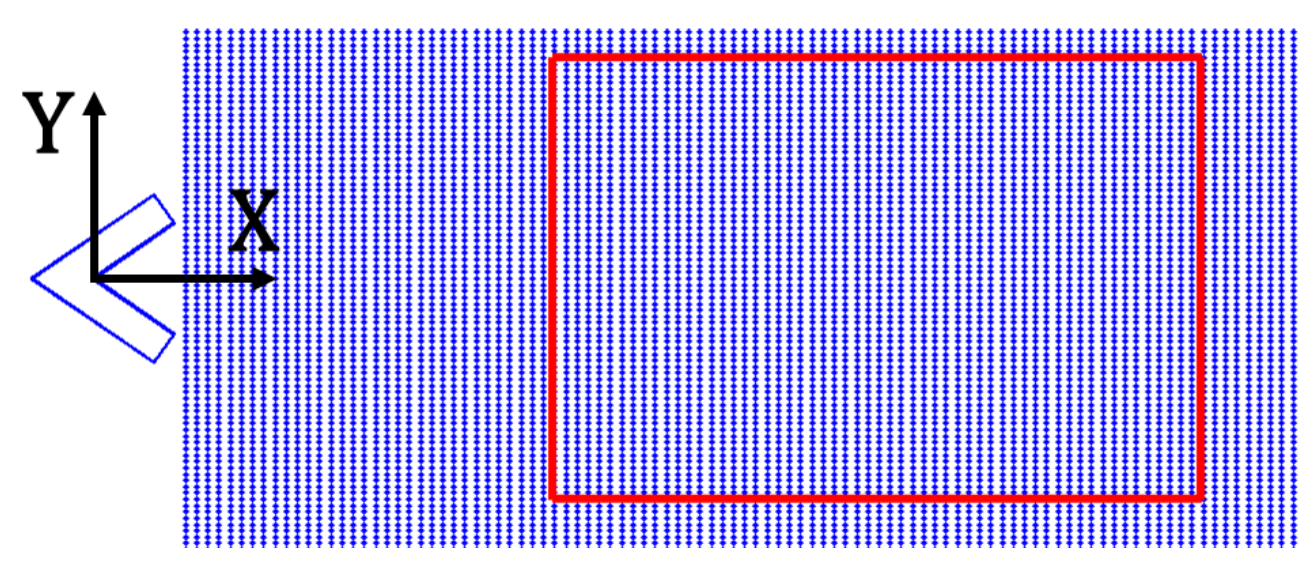

Figure 2.9 - Illustration of relative sizes and locations of sampling planes relative to the flameholder: 100x100 CFD sample grid points (blue) compared to the effective viewing area for the chemiluminescence measurements.

\section{$2.8 \quad$ RESULTS}

A number of simulations were conducted to investigate the effect of various physical and numerical factors on the wake's structure. The investigations began by comparing the time averaged and root-mean-squared data from the chemiluminescence measurements against the projection integral of heat release from the baseline simulation in figures 2.10 and 2.11. These showed similar flames with very low flame angles to the point that the two flames sheets were nearly parallel to one another and roughly symmetric across the horizontal midplane of the experiment. The flame brush in the experiment was somewhat thicker than the simulated flame as evidenced in both the mean and rms data. 

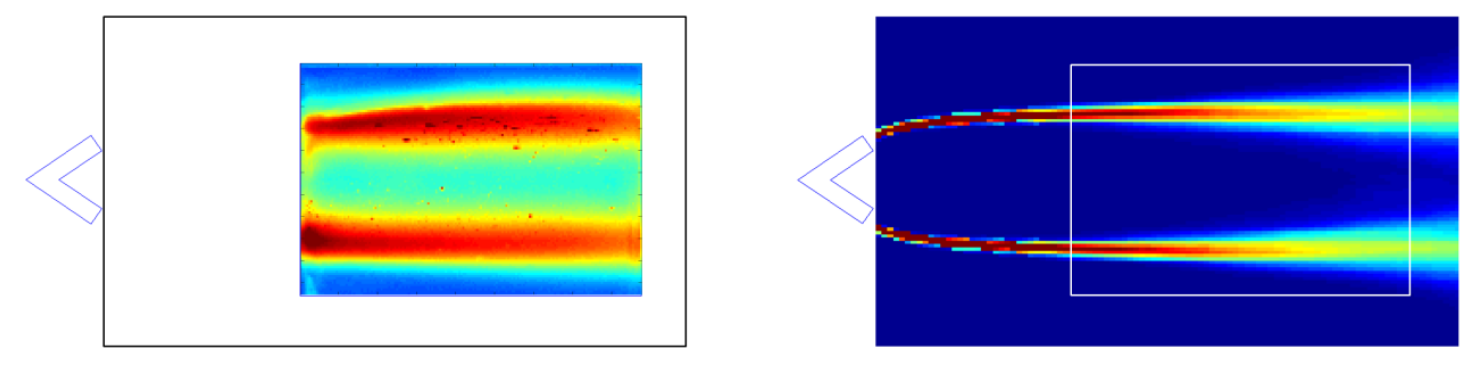

Figure 2.10 - Line of sight mean heat release fields as measured by chemiluminescence (left) and LES (right).
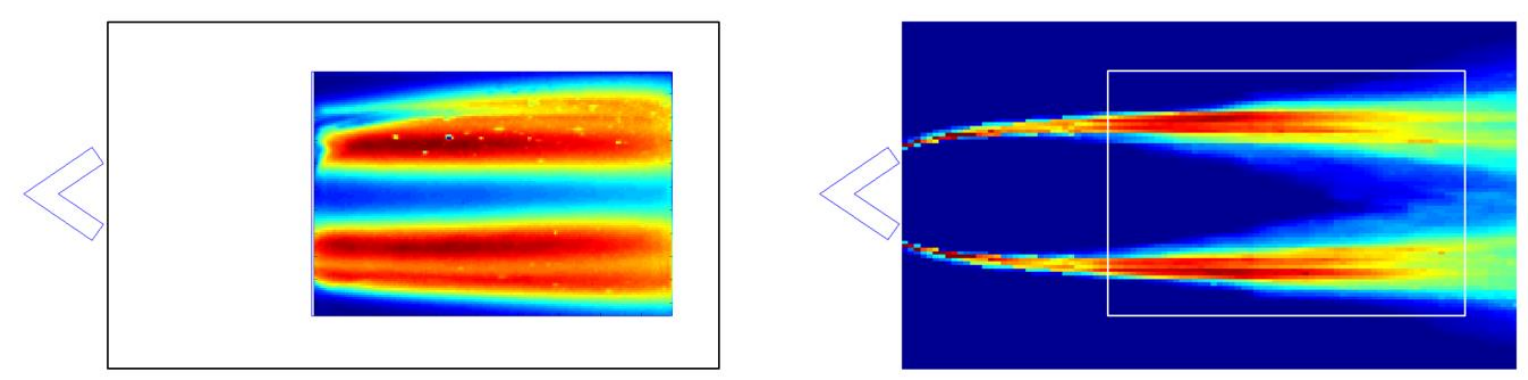

Figure 2.11 - Line of sight RMS heat release fields as measured by chemiluminescence (left) and LES (right).

\subsubsection{Comparison of POD Modes}

As a first comparison between the dynamics of the experimental and numerical experiments, the dominant modes from the chemiluminescence data were compared to those of the baseline simulation in Fluent. Both data sets showed a dominant mode pair of heat release structures in the flame that were roughly symmetric top to bottom and showed good agreement between measurements in terms of their size, shape as shown in figure 2.12. Spectral analysis of the POD coefficients of these modes showed that the Strouhal number of these dominant modes 
differed somewhat with the chemiluminescence data showing Str=0.24 and the Large-Eddy Simulation giving Str=0.29.
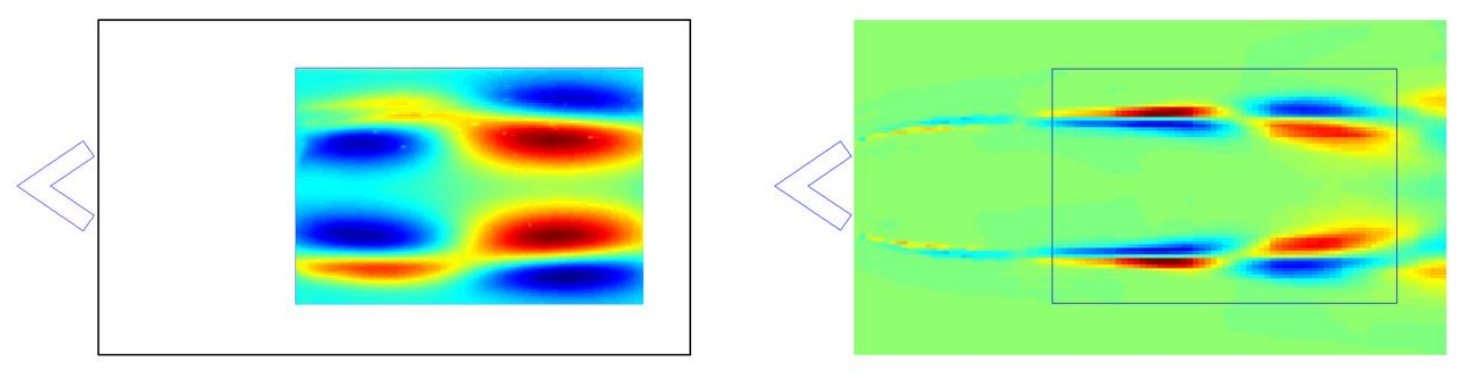

Figure 2.12 - Contours of the dominant POD modes of chemiluminescence (left) and the projection integral of heat release in the LES model (right). The Strouhal numbers of these modes were 0.24 and 0.29 respectively.

The next most energetic modes (modes 3 and 4) of the two decompositions likewise showed good overall agreement judged qualitatively in terms of the shape of the modes as shown in figure 2.13, but also in terms of the spectral content of the POD coefficients where the decomposition of the chemiluminescence data showed a clear peak at $\mathrm{Str}=0.64$ which matched the $S t r=0.63$ from the LES data very closely.
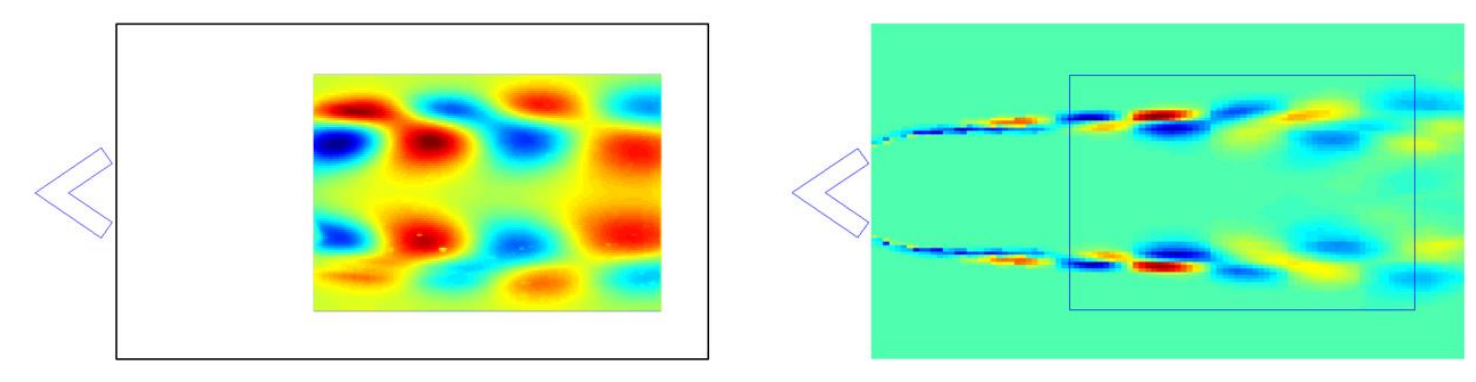

Figure 2.13 - Comparison between mode shapes of the second-most energetic pair of modes showing chemiluminescence data on the left and the baseline LES model on the right. The Strouhal numbers of these modes matched closely between experiment and simulation giving $S t r=0.64$ and $S t r=0.63$ respectively. 
At the higher mode numbers the agreement experiment and simulation degraded significantly, however one interesting result was observed where the $11^{\text {th }}$ mode of the decomposed chemiluminescence data and the $7^{\text {th }}$ mode of the simulation data showed a close match between the modeshapes of a low-frequency oscillation in both the experimental (Str = $0.037)$ and LES flames $(S t r=0.036)$ as demonstrated in figure 2.14 .
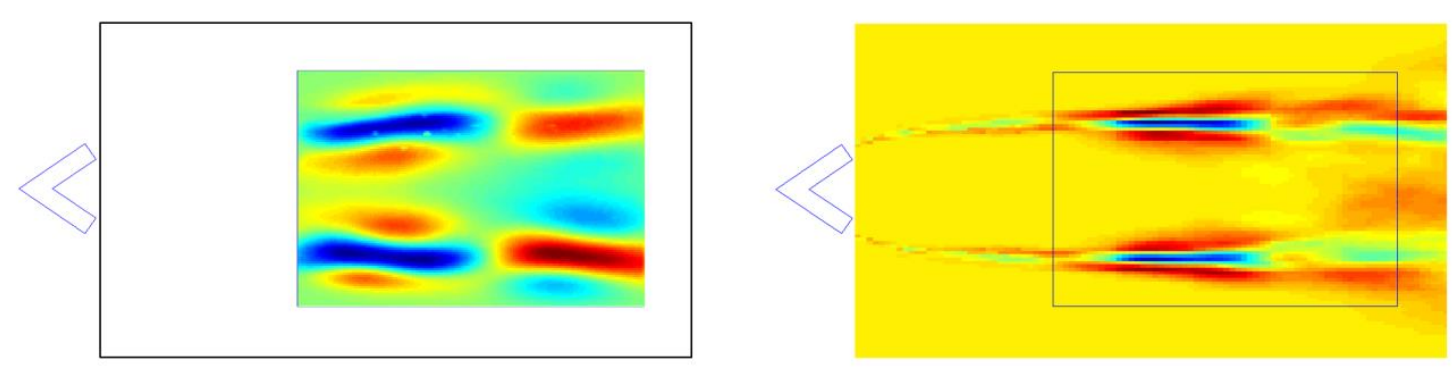

Figure 2.14 - Contours of a low-frequency, low-energy mode revealed during POD of the chemiluminescence (left) and LES data (right). The spectra of these modes' POD coefficients showed peaks at $S t r=0.037$ and $S t r=0.036$ respectively.

\subsubsection{Wall and Midplane Heat Release vs Projection}

To understand how the flame's oscillations varied across the span of the test section, the line-of-sight-integrated heat release signal was compared via POD to the local heat release at the midplane and near-wall (80\% of the distance from the midplane to the sidewall) sampling locations. The results, shown in figure 2.15, show that the dominant modes of the line-of-sightintegrated heat release closely matches the size and shape of the same modes of the midplane local heat release. The nearwall local heat release modes show decent agreement with the integrated baseline, but show slightly more skew and asymmetry in its modeshapes compared to either the midplane or the line-of-sight signal. 

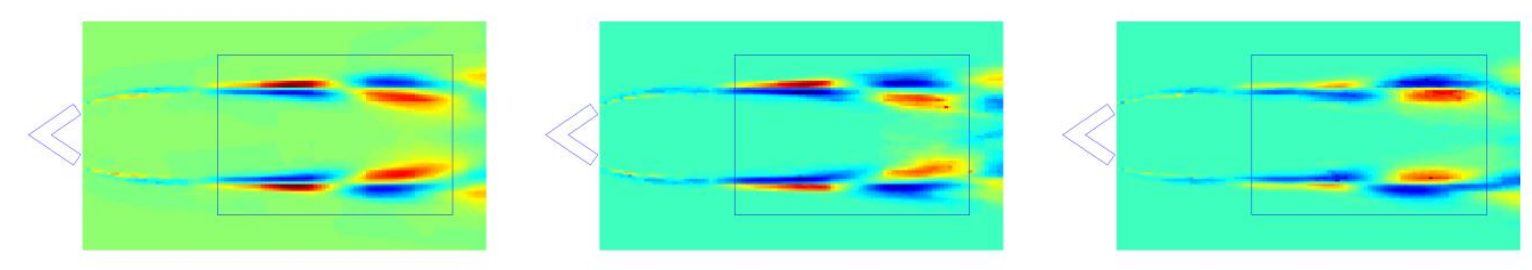

Figure 2.15 - Comparison betwen the dominant modes of the baseline LES model as determined by the line-of-sight-integrated heat release across the full width of the test section (left) and the local heat release at the midplane (middle) and near-wall (right) sampling locations.

\subsubsection{Periodic Side Boundaries}

In an effort to understand how the confinement of the flame within the walls of the testsection might be influencing the flame's dynamics. The baseline simulation was modified by replacing the two sidewalls with boundary conditions with periodicity in the spanwise direction. The dominant modes of the decomposed data from this simulation, compared against those of the baseline simulation in figure 2.16, showed three interesting differences. First, the modified simulation shows strong anti-symmetry top to bottom much more akin to what was observed in the non-reacting case reported previously [55] whereas the baseline simulation and the experiment both showed symmetric modeshapes. Second, the top and bottom flame sheets in the periodic simulation are not parallel as in the confined baseline simulation. Finally, the thickness of the modeshapes became much thicker to more closely match the size range seen in the experiment. 

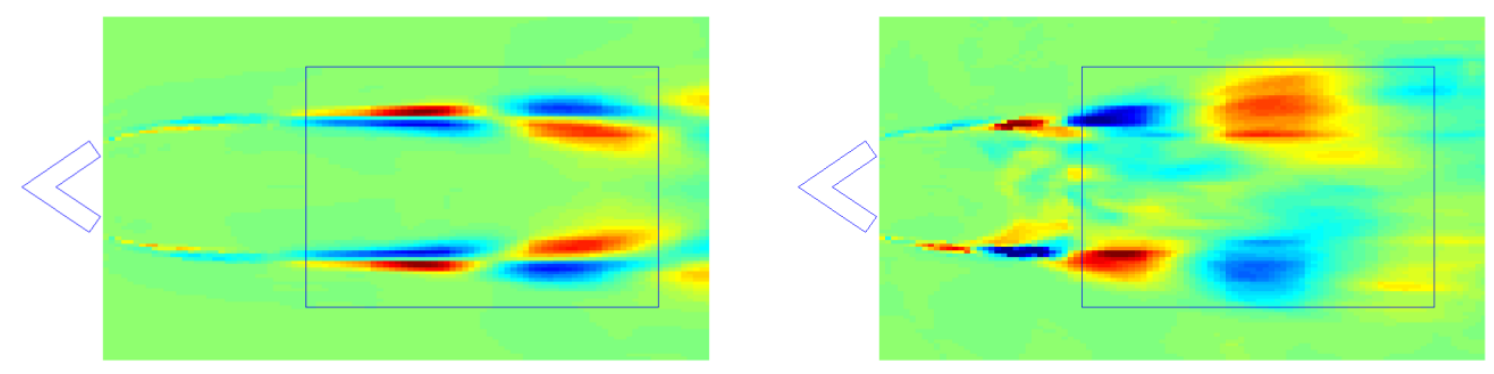

Figure 2.16 - Dominant POD modes of the baseline simulation (left) and simulation with periodic sidewalls (right).

\subsubsection{Thicker Boundary Layer}

To understand how the developing boundary layer in the unburned fuel/air mixture may be affecting the flame's dynamics, the baseline simulation was modified again by extending the domain further upstream such that the inlet plane was relocated from 9.1 vee-gutter widths to $14.1 w$ upstream of the leading edge of the flameholder as shown in figure 2.17.
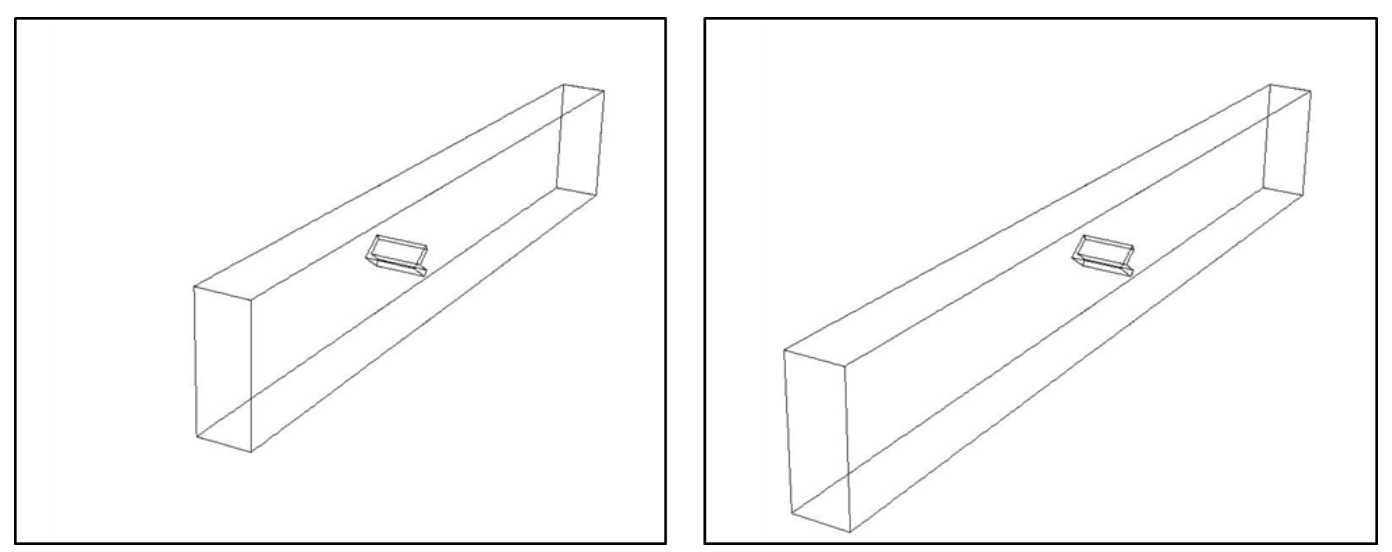

Figure 2.17 - Illustration of the geometry modfication to understand the effect of the boundary layer on the flame's dynamics. The baseline geometry is shown on the left and the extended geometry is shown on the right.

This change in the boundary layer had three effects on the flame's dominant modes. First, the dominant modes of heat release were skewed to the point that the shapes of these modes were 
fully asymmetric top to bottom as shown in figure 2.18. The second and third differences were similar to those seen in the simulation with the periodic sides, namely that the effective flame angle increases and the oscillating modeshapes get thicker to more closely resemble those seen in the experiment.
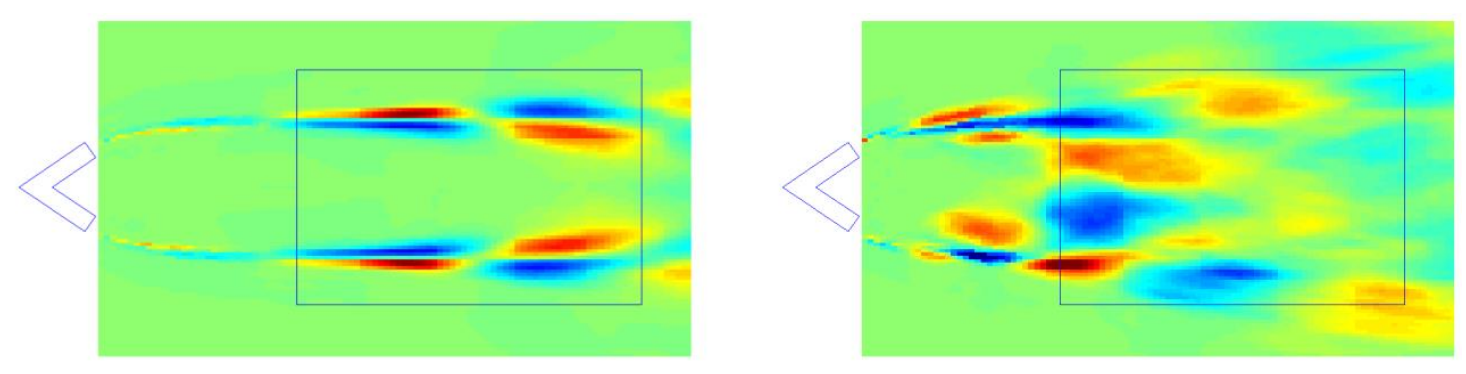

Figure 2.18 - Comparison between dominant POD modes of baseline simulation (left) and simulation with thicker boundary layer (right).

Taken together with the outcomes of the simulation with periodic side boundaries, these results seem to indicate that the near-parallel flame sheets seen in both the experiment and baseline simulation may be a result of the sidewalls' damping of fluctuations. When the sidewalls are removed in the case of the periodic side boundaries or when additional fluctuations are introduced via a thickened boundary layer the result seems to be increased effective flame angles more in line with previous work [14].

\subsection{Conclusions}

The results show how the use of proper orthogonal decomposition was applied to studying the dynamics of a bluff-body-stabilized flame as measured using high-speed chemiluminescence imaging and as simulated using large eddy simulation. The agreement between the shapes and magnitudes of the dominant first modes was overall quite good with this agreement even extending to the second-most dominant pair of modes. While occurring at different mode numbers in the two data sets, a low-energy, low-frequency oscillation seen in the chemiluminescence data was shown to be quite faithfully reproduced by the simulation both in size and shape as well as in frequency content. A numerical study of wall effects on the flame's dynamics showed that changing the sidewalls to periodic boundaries as well as thickening the 
boundary layer near the flameholder, thickened the flame's dynamics modes and destroyed the top-to-bottom symmetry that had been seen in both the baseline simulation as well as in the experiment. 


\begin{tabular}{|c|c|}
\hline POD & Proper Orthogonal Decomposition \\
\hline Str & Strouhal number \\
\hline$f$ & frequency \\
\hline$w$ & Width of vee-gutter bluff body \\
\hline $\mathrm{U}$ & Bulk flow velocity \\
\hline $\operatorname{Re}$ & Reynolds number and similar abbreviations do not use italics \\
\hline$\rho$ & Density \\
\hline$\mu$ & Dynamic viscosity \\
\hline$A$ & Cross-sectional area \\
\hline Ro & Roschko Number \\
\hline Blockage & Blockage Ratio of Bluff Body \\
\hline RHS & Right Hand Side \\
\hline LHS & Left Hand Side \\
\hline$u$ & Snapshot vector of instantaneous data \\
\hline $\boldsymbol{U}$ & Matrix of snapshot vectors \\
\hline C & Correlation Matrix \\
\hline$\lambda$ & Vector of Eigenvalues \\
\hline$P$ & Eigenmode matrix \\
\hline
\end{tabular}


B

$u$

v

$P$

$e$

D

$R$

$T$

$\sigma$

$c_{v}$

V

C

$\Delta$

$\tau$

$k$

$S$

c

SGS

LES
Eigenvector matrix

Velocity, x-direction

Velocity, y-direction

Pressure

Internal energy

Diffusivity

Gas constant

Temperature

Strain rate

Specific heat

Volume

Progress variable

Filter width

Stress

Kinetic energy

Flame Speed

Progress Variable

Subgrid scale

Large eddy simulation 


\subsection{References}

[1] Macquisten, M., and Dowling, A., (1993) "Low Frequency Combustion Oscillations in a Model Afterburner." Combustion and Flame, 94 pp. 253-264.

[2] Giacomazzi, E., Battaglia, V., and Bruno, C. (2004). "The coupling of turbulence and chemistry in a premixed bluff-body flame as studied by LES." Combustion and Flame, 138 (4) pp. 320-335. doi:10.1016/j.combustflame.2004.06.004

[3] Shanbhogue, S. J., Husain, S., and Lieuwen, T. (2009). "Lean blowoff of bluff body stabilized flames: Scaling and dynamics” Progress in Energy and Combustion Science 35 (1) pp. 98-120. doi:10.1016/j.pecs.2008.07.003

[4] Giezendanner, R., Keck, O., Weigand, P., Meier, W., Meier, U., Stricker, W., and Aigner, M., (2003) "Periodic combustion instabilities in a swirl burner studied by phase-locked planar laser-induced fluorescence" Combustion Science and Technology, 175 (4) pp 721-741. doi:10.1080/00102200302390

[5] Roshko, A., (1952) "On the Development of Turbulent Wakes from Vortex Streets" Ph.D. thesis, Mechanical Engineering, California Institute of Technology.

[6] Yang, J.-T., and Tsai, G.-L. (1992). "The Wake Flow Structure of an Open-Slit Vee Gutter" Experimental Thermal and Fluid Science, 5 pp. 685-696.

[7] Karniadakis, E., and Triantafyllou, G., (1992) “Three-dimensional dynamics and transition to turbulence in the wake of bluff objects" Journal of Fluid Mechanics, 238 pp 1-30. [8] Spalart, P. R., and Squires, K. D., (2004) "The status of detached-eddy simulation for bluff bodies. In The Aerodynamics of Heavy Vehicles: Trucks, Buses, and Trains (pp. 29-45). Springer Berlin Heidelberg. 
[9] Shur, M., Spalart, P. R., Squires, K. D., Strelets, M., and Travin, A., (2005). “ThreeDimensionality in Reynolds-Averaged Navier-Stokes Solutions Around Two-Dimensional Geometries" AIAA journal, 43 (6) pp 1230-1242.

[10] Achenbach, E., \& Heinecke, E. (1981). On vortex shedding from smooth and rough cylinders in the range of Reynolds numbers 6x 1. Journal of Fluid Mechanics, 109, 239-251.

[11] West, G. S., \& Apelt, C. J. (1982). The effects of tunnel blockage and aspect ratio on. Journal of Fluid Mechanics, 114 pp. 361-377.

[12] Achenbach, E., and Heinecke, E. (1981). "On vortex shedding from smooth and rough cylinders in the range of Reynolds numbers $6 \times 10^{3}$ to $5 \times 10^{6 \%}$ Journal of Fluid Mechanics, $109 \mathrm{pp}$. 239-251.

[13] Huang, R., and Chang, K. (2004) "Oscillation Frequency in Wake of a Vee Gutter." Journal of Propulsion and Power, 20 (5) pp. 871-878. doi:10.2514/1.9431.

[14] Mattingly, J., (2004) Elements of Gas Turbine Propulsion. New York: McGraw-Hill.

[15] Cocks, P., Sankaran, V., and Soteriou, M., (2013) "Is LES of Reacting Flows Predictive?

Part 1 : Impact of Numerics" 51 $1^{\text {st }}$ AIAA Aerospace Sciences Meeting 2013, pp. 1-29.

[16] Sjunneson, A., Henrikson, P., and Lofstrom, C., (1992) “CARS Measurements and Visualization of Reacting Flows in a Bluff Body Stabilized Flame.” 28th Joint Propulsion Conference and Exhibit 1992, doi:doi:10.2514/6.1992-3650.

[17] Giacomazzi, E., and Bruno, C., (2000) "Effects of Pressure on Unsteady Flame Stabilization"36th AIAA/ASME/SAE/ASEE Joint Propulsion Conference 2000.

[18] Fureby, C., and Lofstrom, C., (1994) "Large-Eddy Simulations of Bluff Body Stabilized Flames." The Combustion Institute: Twenty-Fifth International Symposium on Combustion, pp. $1257-1264$. 
[19] Schadow, K., and Gutmark, E., (1992) “Combustion Instability Related to Vortex

Shedding in Dump Combustors and Their Passive Control." Progress in Energy and Combustion Science, 18 (2) pp. 117-132. doi:10.1016/0360-1285(92)90020-2.

[20] Leibovich, S., (1978) “The Structure of Vortex Breakdown.” Annual Review of Fluid Mechanics, 10 pp. 221-246.

[21] Erickson, R., Soteriou, M., and Mehta, P., (2006) “The Influence of Temperature Ratio on the Dynamics of Bluff Body Stabilized Flames." 44th AIAA Aerospace Sciences Meeting and Exhibit 2006, pp. 1-18.

[22] Stein, O., and Kempf, A.. "LES of the Sydney Swirl Flame Series: A Study of Vortex Breakdown in Isothermal and Reacting Flows" Proceedings of the Combustion Institute 31 (2) pp. 1755-1763. doi:10.1016/j.proci.2006.07.255.

[23] Ranalli, J., (2009) "Spatially Resolved Analysis of Flame Dynamics for the Prediction of Thermoacoustic Combustion Instabilities,” Ph.D. thesis, Mechanical Engineering, Virginia Tech. [24] Haber, L.C., (2000) “An Investigation into the Origin, Measurement and Application of Chemiluminescent Light Emissions from Premixed Flames” M.S. thesis, Mechanical Engineering, Virginia Tech.

[25] Farina, J., (2013) “Application of Multi-Port Mixing for Passive Suppression of ThermoAcoustic Instabilities in Premixed Combustors" Ph.D. thesis, Mechanical Engineering, Virginia Tech.

[26] Wickersham, A.J., Xuesong L., and Ma, L., (2014) “Comparison of Fourier, Principal Component and Wavelet Analyses for High Speed Flame Measurements." Computer Physics Communications 185, (4) pp. 1237-1245. doi:10.1016/j.cpc.2013.12.022. 
[27] Smagornisky, J., (1963) "General Circulation Experiments with the Primitive Equations," Mon. Weather Rev., 91 (3) pp. 99-164.

[28] Germano, M., Piomelli, U., Moin, p., and Cabot, W., (1991) “A dynamic subgrid-scale eddy viscosity model," Physics of Fluids and Fluid Dynamics., 3 (7) pp. 1760.

[29] Lilly, D., (1992) “A proposed modification of the Germano subgrid-scale closure method," Physics of Fluids and Fluid Dynamics., 4 (3) pp. 633.

[30] Butler, T., and O’Rourke, P., (1977) “A Numerical Method for Two Dimensional Unsteady Reacting Flows.” Symposium (International) on Combustion, 16 (1) pp. 1503-1515. doi:10.1016/S0082-0784(77)80432-3.

[31] Selle, L., (2004) "Compressible Large Eddy Simulation of Turbulent Combustion in Complex Geometry on Unstructured Meshes." Combustion and Flame, 137 (4) pp. 489-505. doi:10.1016/j.combustflame.2004.03.008.

[32] Poinsot, T., and Veynante, D., (2009) Theoretical and Numerical Combustion, RT Edwards.

[33] Huang, Ying, and Vigor Yang. "Dynamics and Stability of Lean-Premixed SwirlStabilized Combustion." Progress in Energy and Combustion Science, 35 (4) pp. 293-364. doi:10.1016/j.pecs.2009.01.002.

[34] Zimont, V., Polifke, W., Bettelini, M., and Weisenstein, W., (1998) “An Efficient Computational Model for Premixed Turbulent Combustion at High Reynolds Numbers Based on a Turbulent Flame Speed Closure" Journal of Engineering for Gas Turbines and Power, 120 pp. $526-532$. 
[35] Zimont, V., Biagioli, F., and Syed, K., (2001) "Modelling Turbulent Premixed

Combustion in the Intermediate Steady Propagation Regime." Progress in Computational Fluid Dynamics, 1 (1) pp. 14-28.

[36] Lamoureux, N., Djebaïli-Chaumeix, N., and Paillard, C., (2003) "Laminar Flame Velocity Determination for H2-air-He-CO2 Mixtures Using the Spherical Bomb Method." Experimental Thermal and Fluid Science, 27 (4) pp. 385-393. doi:10.1016/S08941777(02)00243-1.

[37] Pearson, K. (1901). On lines and Planes of Closest Fit to Systems of Points in Space. Philosophical Magazine, 2 pp. 559-572.

[38] Lumley, J., (1967) “The Structure of Inhomogeneous Turbulent Flows,” Atmospheric Turbulence and Radio Wave Propagation, 114 pp. 166-178.

[39] Gergen, I., and Harmanescu, M. (2012), “Application of principal component analysis in the pollution assessment with heavy metals of vegetable food chain in the old mining areas" Chemistry Central Journal, 6 (1) pp 156. doi:10.1186/1752-153X-6-156

[40] Smith, T., Moehlis, J., and Holmes, P., (2005) "Low-Dimensional Modelling of Turbulence Using the Proper Orthogonal Decomposition: A Tutorial," Nonlinear Dynamics, 41 (1-3) pp. 275-307.

[41] Sirovich, L., and Kirby, M., (1987) "Low-dimensional procedure for the characterization of human faces," Journal of the Optical Society of America, 4 (3) pp. 519.

[42] Hsieh, T., Chen, J., Chen, L., Chiang, P., and Lee, H., (2011) “Time-course gait analysis of hemiparkinsonian rats following 6-hydroxydopamine lesion.," Behavioral Brain Research, 222 (1) pp. 1-9. 
[43] Berkooz, Gal, Holmes, P., and Lumley, J., (1993) “The Proper Orthogonal

Decomposition in the Analysis of Turbulent Flows.” Annual Review of Fluid Mechanics 25, no.

(1) pp. 539-575. doi:10.1146/annurev.fl.25.010193.002543.

[44] Fukunaga, K. (1990). Introduction to statistical pattern recognition. Academic press. p. 592.

[45] Moin, P., and Moser, R., (2006) "Characteristic-eddy decomposition of turbulence in a channel," Journal of Fluid Mechanics 200 pp. 471.

[46] Sirovich, L., and Kirby, M., (1987) "Low-dimensional procedure for the characterization of human faces," Journal of the Optical Society of America, 4 (3) pp. 519.

[47] Duwig, C., and Iudiciani, P., (2009) "Extended Proper Orthogonal Decomposition for Analysis of Unsteady Flames,” Flow, Turbulence, Combustion, 84 (1) pp. 25-47.

[48] Böhm, B., Brübach, J., Ertem, C., and Dreizler, A., "Experiments for Combustion-LES Validation," Flow, Turbulence, Combustion, 80 (4) pp. 507-529.

[49] Miranda, A., Le Borgne, Y., and Bontempi, G., (2007) "New Routes from Minimal Approximation Error to Principal Components," Neural Process Letters, 27 (3), pp. 1-14. [50] Chatterjee, P., (2004) “A Computational Fluid Dynamics Investigation of Thermoacoustic Instabilities in Premixed Laminar and Turbulent Combustion Systems,” Ph.D. thesis, Mechanical Engineering, Virginia Tech.

[51] Chishty, W., (2005) "Effects of Thermoacoustic Oscillations on Spray Combustion Dynamics with Implications for Lean Direct Injection Systems Effects" Ph.D. thesis, Mechanical Engineering, Virginia Tech. 
[52] Hendricks, A., (2003) "Determination of Flame Dynamics for Unsteady Combustion Systems using Tunable Diode Laser Absorption Spectroscopy" Ph.D. thesis, Mechanical Engineering, Virginia Tech.

[53] Vukadinovic, V., Habisreuther, P., and Zarzalis, N., (2010) "Experimental study on the influence of pressure and temperature on the burning velocity and Markstein number of jet A-1 kerosene" ASME Turbo Expo 2010.

[54] ANSYS FLUENT Documentation v 12.0, 2009.

[55] Blanchard, R., Ng, W., Lowe, K., and Vandsburger, U., (2014) "Simulating Bluff Body Flameholders: On the Use of Proper Orthogonal Decomposition for Wake Dynamics Validation." ASME Journal of Engineering for Gas Turbines and Power (2014). 


\section{Paper \#3 - Study of Temperature Ratio Effects on Bluff Body Wake Dynamics Using Large Eddy Simulation and Proper Orthogonal Decomposition}

\subsection{Abstract}

In this article, we describe the use of proper orthogonal decomposition (POD) to investigate how the dominant structures of a turbulent premixed flame change as a function of the amount of heat released in a bluff-body-stabilized turbulent premixed flame. The investigation uses a validated large eddy simulations (LES) to numerically model the flame's dynamics [1-2]. The numerical simulations allow the effect of heat release to be decoupled from the laminar flame speed. Five simulations are reported here that vary the amount of heat released in the combustion reaction between 0 and $100 \%$ of the stoichiometric value, quantified by the temperature ratio of the burned to unburned gases, while holding constant both the laminar flame speed as well as the method of turbulent flame speed closure. The results indicate similar trends reported qualitatively by others, but by using POD to isolate the dominant heat release modes of each simulation, the decomposed data can clearly show how the previously-reported flow structures transition from asymmetric shedding in the case of zero heat-release to a fully symmetric shedding mode in the case of full heat release.

\subsection{Introduction}

The unsteady release of sensible heat and flow dilation in combustion processes create pressure fluctuations which, particularly in premixed flames, can couple with the acoustics of the combustion system. This acoustic coupling creates a feedback loop with the heat release that amplifies to thermo-acoustic instabilities which can reduce combustion efficiency, increase pollutant formation, and even damage the combustor. For vee-gutter-type bluff-body flameholders, the flame is stabilized by recirculating hot combustion products from the veegutter's wake. This makes characterization of the vee-gutter's wake dynamics very important. Not only for understanding the flameholder's performance in the wet (fueled) mode but in the dry (unfueled) mode as well since many of the afterburner's performance characteristics like ignition, turndown, lean blowout limit, and dry loss feature either substantially reduced reaction rates or no reactions at all. Accordingly, this study was conducted to investigate how the wake transitions from an asymmetric shedding-type flow structure when not reacting, to a symmetric structure in the presence of the flame. 
Numerical simulations of bluff-body flameholders, in either wet or dry operation, are often validated against experimental measurements using time-averages and point-wise statistics to evaluate the simulations predictive capabilities; however these techniques fail to capture the combined spatial and temporal nature of the dynamics of these systems. This work uses proper orthogonal decomposition (POD) to analyze both experimental and simulated data to isolate and then quantitatively compare the shapes and amplitudes of the dynamic modes of each against one another.

To enhance the comparison between the numerical simulations and the experimental measurements of the flame's chemiluminescent emissions, which is a line-of-sight type of measurement, the simulations were post processed by integrating the source term of the dimensionless combustion reaction along simulated lines of sight toward the position relative to the flame when the measurements were made. These line-integrated signals, once decomposed into its primary components using POD, formed the basis of comparison between the different sets of data.

\subsection{Review of Literature}

Significant efforts to validate combustion LES against experiments have been reported in the literature. These consist largely of comparisons between measured and simulated timeaverages, Reynolds decompositions, and power spectral density as validation metrics [3-6]. While time averages and power spectra do represent a natural and intuitively meaningful basis for validation, and instantaneous snapshots of temperature or velocity do provide qualitative characterization of the dominant flow structures, they are insufficient for quantitative comparisons of the size and shape of a highly non-linear dynamic system such as a turbulent flame. One approach which has been used to attempt to solve this problem is phase-locked sampling, which has been successfully used to study flames with periodic instabilities [7]. This technique however requires an external forcing source which can be used to trigger the measurement which prevents it from being applied to self-excited flames. Most commonly the spatial arrangement of dominant flow features are shown through instantaneous [6]. Such snapshots are clearly valuable for qualitative validation but cannot be quantitatively compared to similar snapshot from a different system and can obscure less-dominant flow features which may be of importance but are overshadowed by the more dominant structures. 
Decomposition techniques, such as proper orthogonal decomposition (POD) allow many of these shortcomings to be overcome for stationary processes and allow for reproducible, statistically converged representations of the key flow features and their various magnitudes, time scales, and spatial arrangements. Ensembles of these instantaneous snapshots can be decomposed in space and/or time to separate the various coherent modes from each other in a way that allows for a statistically representative, reproducible, and quantitative description of each. This technique finds linear combinations of instantaneous snapshots of the system to 'filter' out and isolate the individual dynamic structures from each other [8]. Because such decompositions rely only on the input data, and require no underlying model or approximation, they make no distinction between data generated by a physical measurement or a numerical simulation. This makes these techniques well suited to the comparisons of measured and simulated dynamics shown here.

The effect of dilation on vorticity can be seen in the vorticity equation for variable density flows is shown as equation 1 . The left hand side (LHS) of this equation contains standard terms for the unsteady convection of vorticity, but the right hand side (RHS), particularly its first two terms which are not present in flows with constant density, are significant for understanding the dynamics of such flows. The first term on the RHS represents volumetric expansion due to heat release which effectively acts as a vorticity sink by expanding velocity gradients to a longer length scale as than the original, higher density of its unburned state. This term is very important to the bluff-body problems considered here as the flame exists in a shear layer which would normally exhibit higher vorticity due to the velocity deficit in the bluff body wake but which, due to the dramatic density drop for fluid crossing flame and shear layer, is substantially reduced. The second term which governs the baroclinic production of vorticity, is less important for the current study since the pressure gradient and the density gradient are roughly aligned perpendicular along the shear layer, but may be of greater importance for other (e.g. swirling) burners.

$$
\frac{\partial \vec{\omega}}{\partial t}+(\vec{u} \cdot \nabla) \vec{\omega}=-\vec{\omega}(\nabla \cdot \vec{u})+\frac{\nabla \rho \times \nabla p}{\rho^{2}}+(\vec{\omega} \cdot \nabla) \vec{u}
$$




\subsubsection{Proper Orthogonal Decomposition}

Proper orthogonal decomposition (POD), is a data analysis technique originally proposed in 1901 (Pearson) [8] and first recognized by Lumley [9] for its value in analyzing turbulent flows. The statistical technique has been applied to data in many different fields that has been applied to a wide range of problems from pollutant dispersion [10] to reduced-order modeling [11] to machine vision [12] to neurology [13]. Owing in part to the diversity of its applications, POD is also referred to by a number of names including: principal component analysis, the Karhunen-Loève transformation, the method of empirical orthogonal functions, singular-value decomposition, eigenvalue decomposition, factor analysis, the Eckart-Young theorem, empirical component analysis, the Hotelling transform, among others. The method is essentially a pattern recognition technique that seeks to approximate a dataset through a linear combination of a minimum number of orthogonal vectors [14].

While limited in aspects in its ability to discriminate between data classes [15], the proven application of POD in detecting coherent structures in turbulent [16 - 17] and reacting flows [18 - 21], together with its agnosticism toward the source of data, makes this technique particularly well-suited to the comparison of simulated and measured combustion dynamic data.

The method for calculating the POD begins by compiling a "snapshot' of sample data, either measured or simulated, into column vector $\mathbf{u}^{\mathbf{i}}$ in equation 3.2, these vectors are then assembled into an MxN matrix $\mathbf{U}$ in equation 3.3 where $\mathrm{M}$ is the number of samples in each snapshot and $\mathrm{N}$ is the number of snapshots .

$$
\begin{aligned}
& \boldsymbol{u}^{i}=\left[\begin{array}{c}
u_{1}^{i} \\
u_{2}^{i} \\
u_{3}^{i} \\
\vdots \\
u_{m}^{i}
\end{array}\right] \\
& \boldsymbol{U}=\left[\begin{array}{lllll}
\boldsymbol{u}^{1} & \boldsymbol{u}^{2} & \boldsymbol{u}^{3} & \cdots & \boldsymbol{u}^{N}
\end{array}\right]
\end{aligned}
$$

In the analyses shown here where a snapshot contains both velocity and chemiluminescence data, each element of the vector is appropriately non-dimensionalized to 
prevent inconsistencies in units and to prevent the POD from being biased towards the type of data with numerically larger values. The velocities were normalized by the bulk flow velocity of the given data and the chemiluminescence pixels were normalized by the temporally and spatially averaged value of all such pixels. The mean of each row in $\boldsymbol{U}$ is then subtracted from each element of $\boldsymbol{U}$ [49]. Next, the correlation matrix $\widetilde{\boldsymbol{C}}$ is calculated by premultiplying $\boldsymbol{U}$ by its transpose and dividing by $\mathrm{N}$. Then the eigenvalue problem of $\widetilde{\boldsymbol{C}}$ is solved to find the vector of eigenvalues, $\lambda$, and the matrix of eigenvectors, $\boldsymbol{B} . \boldsymbol{U}$ and $\boldsymbol{B}$ are then multiplied to produce the matrix of unweighted eigenmodes $\boldsymbol{P}$. The properly weighted eigenmodes are calculated by multiplying each unweighted eigenmode with its corresponding eigenvalue.

Where POD is often used to construct a reduced-order model of a system by summing the most energetic modes, there are essentially two applications here, both of which treat each mode (or pair of modes in the case of travelling waves which form sin/cosine pairs) as an isolated physical phenomenon which represents a coherent structure in the data. First, each structure can be used as a means of system identification to qualitatively characterize the system. Second, the properly weighted modes can form the basis for quantitative (in both magnitude and spatial distribution) comparison between similar phenomena isolated from decompositions of different datasets to determine to what extent the two systems share a mode of given dynamic behavior.

\subsection{Experiment}

The experimental data used to validate the LES models at the 0 and $100 \%$ temperature ratio conditions of this study were taken in a model augmentor test rig. The lab features a Kaeser FS440 air compressor and Gaumer electrical heater capable of delivering air up to $0.7 \mathrm{~kg} / \mathrm{s}, 10$ atm, and $650 \mathrm{~K}$.

The rig consisted of three sections: a fuel-injection section, a converging section, and finally the test section. The test-section features a $12.7 \mathrm{~cm} \times 5 \mathrm{~cm}$ rectangular cross-section, and film-cooled windows for optical access just downstream of the flameholder. The rig is designed primarily to burn liquid fuels and has a fuel delivery system capable of supplying $7.5 \mathrm{lpm}$ of liquid fuel.

The vee-gutter flameholder has a constant, vee-shaped cross-section spanning horizontally across the minor axis of the test section. The vee gutter is $6 \mathrm{~mm}$ thick with a tip-to- 
tip dimension of $30.5 \mathrm{~mm}$ giving a blockage ratio of 0.24 ; the included angle of the vee gutter is 70 degrees so as to be representative of typical afterburner configurations.

The test-section features three windows, a pair of side windows measuring $9 \times 13 \mathrm{~cm}$ (3.5" $\times 5$ ") and a single top window measuring $2.5 \mathrm{~cm} \times 13 \mathrm{~cm}$ as shown in figure 3.1 . The leading edges of all three windows are located $6 \mathrm{~cm}$ downstream of the trailing edge of the vee-gutter.

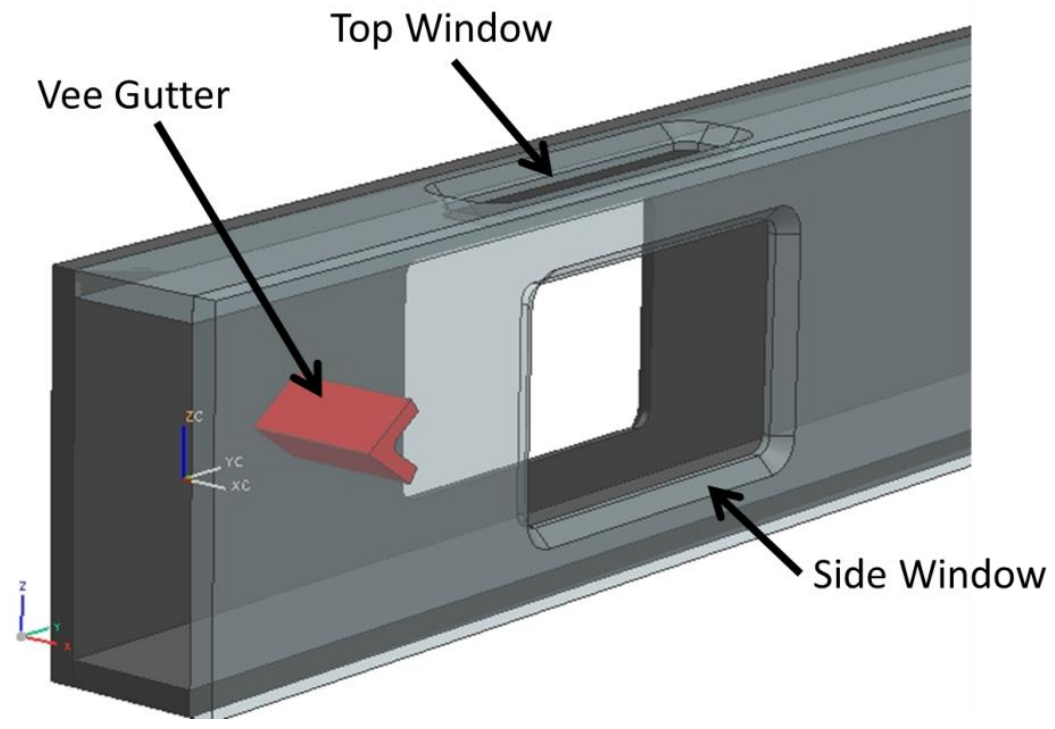

Figure 3.1 - Transparent view of CAD model of the test section showing the locations of the three windows relative to the vee-gutter flameholder. Flow direction is left-to-right.

\subsubsection{Particle Image Velocimetry}

Fluid velocities in the experiment were measured using a LaVision Flowmaster PIV system with a camera resolution of 2048 x 2048 for resolving u- and v-velocity components on a vertical plane halfway between the test section's two side walls as shown in figure 3.2. The $1 \mu \mathrm{m}$ $\mathrm{TiO}_{2}$ seed particles were illuminated by a dual-cavity frequency-doubled 532nm Nd:YAG laser. The DaVis 7 software provided by LaVision was used for initial pre-processing of the images, for compiling the autocorrelations to recover the fields of velocity vectors, and for some postprocessing of the vector fields to remove spurious data. Seeding of the flow was accomplished by injecting $1 \mu \mathrm{m} \mathrm{TiO}_{2}$ particles dispersed using a swirling fluidized bed seeder and injected through a simple tube in a port in the rig's converging section located $30 \mathrm{~cm}$ upstream of the test section. 


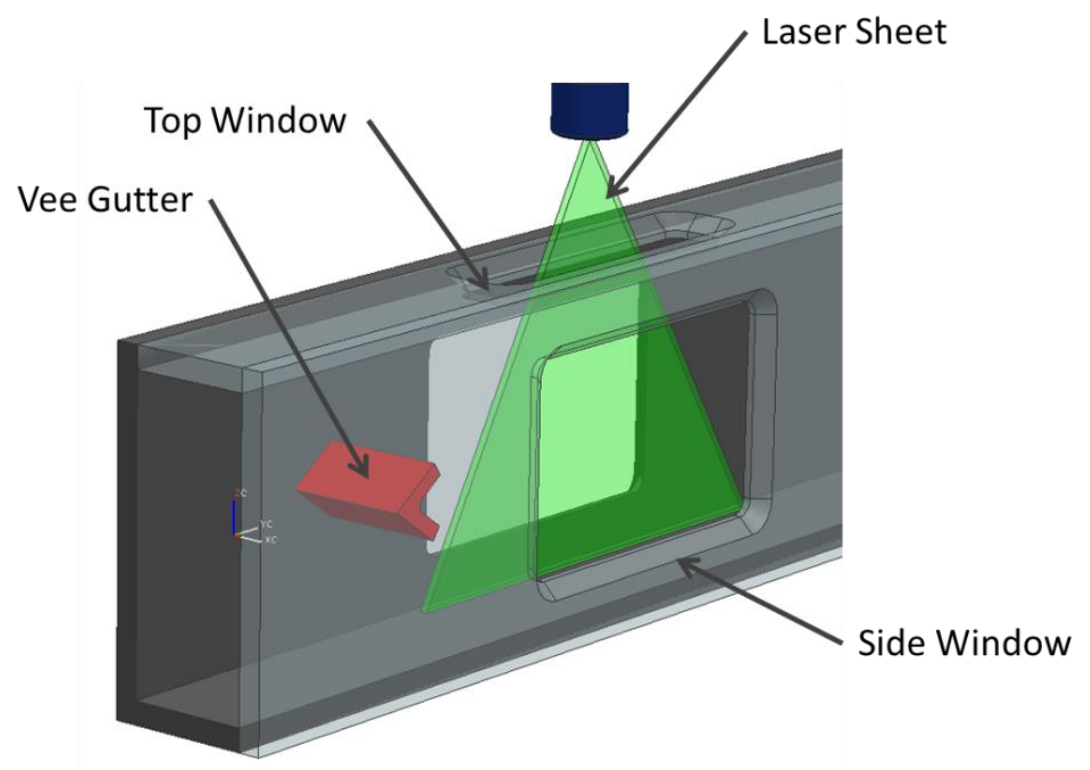

Figure 3.2 - Transparent view of CAD model of the test section showing the locations of the three windows relative to the vee-gutter flameholder and the PIV laser sheet. Flow direction is left-to-right.

\subsubsection{Chemiluminescence}

To capture the spatial and temporal dynamics of the flame, the emissions of the $\mathrm{CH}^{*}$ excited intermediate species were imaged with a high-speed camera. The $\mathrm{CH}^{*}$ chemiluminescence, produced within the flame during oxidation of $\mathrm{C}_{2} \mathrm{H}$ by molecular oxygen [22], emits within a narrow band of wavelengths around 430nm and has been successfully used to quantify unsteady heat release in premixed flames [23 - 24]. These emissions were filtered from the rest of the spectrum with a $430 \mathrm{~nm}+/-10 \mathrm{~nm}$ bandpass filter before being imaged by a Photron Fastcam SA-4 high speed camera at a frequency of $5 \mathrm{kHz}$. These measurements have been successfully used to extract quantitative frequency information from transient and unsteady combustion processes [25].

\subsection{Premixed Combustion Simulations}

Large Eddy Simulation (LES) of fluid flows has been widely applied to turbulent flow for many years across a wide range of applications by filtering the governing equations below the scales of motion that can be adequately resolved by the numerical grid. The variables that are 
resolved by the grid are referred to as the filtered variables and the effect of the fluid motions too small to be captured directly is modeled in the equations of motion of the filtered variables as shown in the filtered continuity, momentum, and energy equations shown below as equations 3.4 - 3.8. In work shown here, the commercial CFD code Fluent was used as the simulation platform to integrate these equations. The velocity, density, temperature, variables were discretized using a bounded second order central scheme in space and an implicit, second-order scheme in time.

$$
\begin{gathered}
\frac{\partial \bar{\rho}}{\partial t}+\nabla \cdot(\bar{\rho} \overrightarrow{\vec{u}})=0 \\
\frac{\partial \bar{\rho} \overrightarrow{\vec{u}}}{\partial t}+\nabla \cdot(\bar{\rho} \overrightarrow{\bar{u}} \overrightarrow{\bar{u}})=-\nabla \bar{P}+\nabla \cdot\left(\sigma_{i j}\right) \\
\frac{\partial \bar{\rho} \bar{e}}{\partial t}+(\overrightarrow{\bar{u}} \cdot \nabla) \bar{e}=\nabla \cdot\left(-\bar{\rho} \overrightarrow{\bar{u}} \bar{P}+\bar{\rho} D_{t h} \nabla \bar{e}\right)+\dot{q} \\
\bar{P}=\bar{\rho} R \bar{T} \\
\bar{e}=c_{v} \bar{T}
\end{gathered}
$$

Clearly the success of the LES approach will be dependent on the how these subgrid scale quantities are modeled. In this work, the SGS models used in the momentum equations are all adapted from those originally proposed by Smagorinsky, which invokes the Boussinesq hypothesis, namely that the effect of the eddy motion can be modeled as an increased turbulent viscosity which is added to the molecular viscosity of the working fluid. Smagorinsky's SGS model, given in equation 3.9, models this viscosity as proportional to the magnitude of the local resolved strain rate, $\dot{\sigma}$, as calculated from then filtered velocity field, and proportional to the square of the filter width, $\Delta$, which is calculated as the cube-root of the volume of the computational shell as shown in equation 3.10. From this model the shear stress tensor can be calculated, as in equation 3.12, demonstrating that the turbulence model, at least for the momentum equation is now closed. The value of the subgrid grid viscosity coefficient, $\mathrm{C}_{\mathrm{S}}$ in equation 3.11, is calculated based on estimates of the local unresolved stresses [26 - 28].

$$
\begin{gathered}
\mu_{\Delta}=\bar{\rho}\left(C_{s} \Delta\right)^{2}|\dot{\sigma}| \\
\Delta=\sqrt[3]{V_{\text {Cell }}}
\end{gathered}
$$




$$
\bar{\tau}_{i j}=\mu_{\Delta}\left[\frac{\partial \bar{u}_{i}}{\partial x_{j}}+\frac{\partial \bar{u}_{j}}{\partial x_{i}}-\frac{2}{3} \frac{\partial \bar{u}_{k}}{\partial x_{k}} \delta_{i j}\right]
$$

To account for the behavior of the turbulent premixed flame within the LES framework, a progress variable approach is used. This method is well-suited to the work here as it attempts to collapse the combined effects of chemistry into a progress variable, c, which regards the state of a fluid particle as number between 0 and 1 which corresponds to 'unburned' and 'burned' conditions respectively. This progress variable propagates from the burned gas as governed by equation 3.12 where $S_{\mathrm{T}}$ is the turbulent flame speed which requires additional closure terms.

$$
\frac{\partial \rho c}{\partial t}+(\vec{u} \cdot \nabla) \rho c=\nabla \cdot\left(\rho D_{t h} \nabla c\right)+S_{T}|\nabla c|
$$

The phenomenological processes that premixed flames exhibit under different flow conditions present a number of problems when attempting to model them numerically since an accurate model must consider the effects of compressibility, turbulence, chemistry, heat release, and the several interactions between them. The problem becomes yet more complicated by the numerical grid used to discretize the flow domain, the individual computational cells of which are often larger in size than the thickness of the flame. To overcome this problem, a thickenedflame approach has been successfully used to represents the flame's processes as a filtered, subgrid scale (SGS) phenomena [29 - 31]. With this treatment the flame is thickened to the length scale of the local grid resolution regardless of the disparity between the two length scales which in turn changes the effective Damkohler and Karlovitz numbers, which can change the dynamic character of the flame structure [32].

The turbulent flame speed closure used in this work is the model by Zimont [33 - 34] to account for the interaction between the flame's resolved and unresolved chemical and turbulent timescales. Shown equations 3.13 and 3.14, this model takes its estimates for the unresolved scales of turbulence directly from the subgrid scale turbulence model, in this case dynamic Smagorinsky. The length- and timescales of the flame's laminar chemistry calculated based on the thermal diffusivity of the unburned mixture, $\alpha$, and the laminar flamespeed, $S_{l}$. Rather than simulating the laminar flamespeed numerically, it is taken as a model input from experimental 
measurements which, due to the complexity of detailed chemistry in hydrocarbon flames, is more reliable than simulations of such [35].

$$
\begin{gathered}
S_{T}=B u^{\prime-3 / 4} S_{l}^{1 / 2}\left(\frac{C_{S} \Delta}{\alpha}\right)^{1 / 4} \\
u^{\prime}=C_{S} \Delta \sqrt{S_{i j} S_{i j}}
\end{gathered}
$$

\subsubsection{Domain, Mesh, and Boundary Conditions}

The computational domain used for all of the simulations shown here consisted of a constant rectangular cross-section duct a prismatic $70^{\circ}$ degree included angle vee-gutter, with a $3.05 \mathrm{~cm}$ tip to tip measurement ' $w$ ', extending through the minor axis of the duct. The vee-gutter has a thickness of $0.21 w$. As shown in figure 3.3, the domain extends $9.1 \mathrm{w}$ upstream of the veegutter's leading edge which is actually longer than the test section itself however this length was chosen based on boundary layer analysis to account for the boundary layer in the transition section that exists prior to the flow entering the test section. The domain is $24.1 \mathrm{w}$ long overall, as shown in figure 3.4, and maintains a rectangular $1.67 w \times 4.17 w$ cross-section throughout. The real experiment also has a constant cross-sectional area, but begins to transition to a hexagonal cross-section at the end of the water-jacket section, this geometrical difference was assumed negligible. 


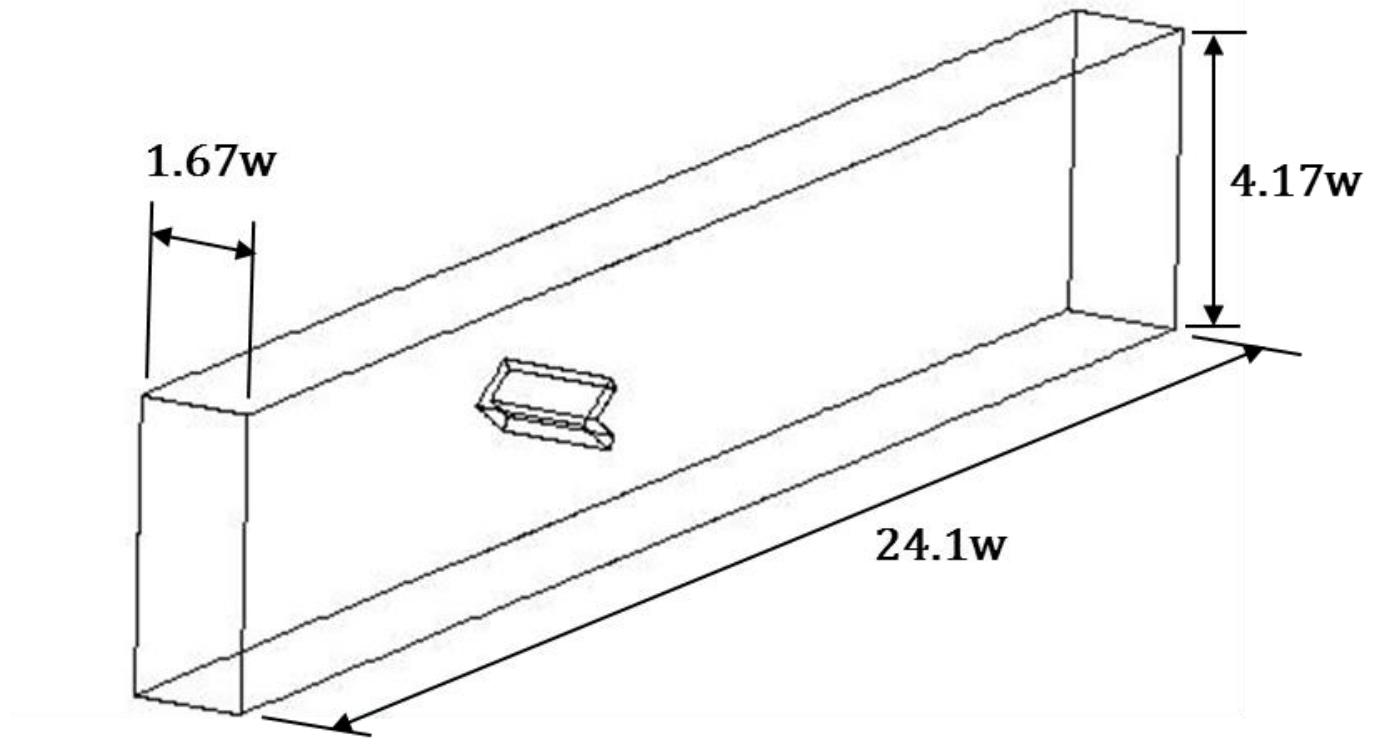

Figure 3.3 - Isometric view of dimensions of the domain used in the LES models based on the width of the flameholder $\mathrm{w}=3.05 \mathrm{~cm}$ and an included angle of $70^{\circ}$.

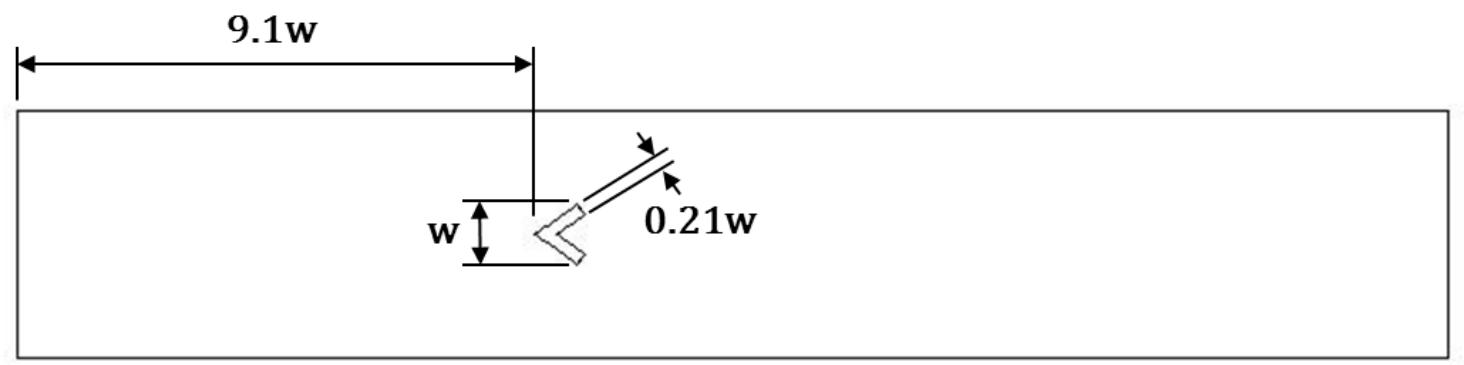

Figure 3.4 - Plan view dimensions of the domain used in LES models.

The mesh used in these simulations consisted of $2.8 \mathrm{M}$ cells, and built in a blockstructured format using ICEM Hexa and then converted to the unstructured formats used by the ANSYS Fluent solver. In making the mesh, care was taken to limit the growth rate in any direction to 1.05 or smaller and the maximum aspect ratio of any cell in the region of interest was limited to 5 or less. Downstream of the region of interest, the aspect ratios of the cells were intentionally stretched in the streamwise direction to dampen out fluctuations in the flow before they reached the outlet boundary. 
The boundary conditions for all five of the simulations reported here were identical except for the amount of heat released by the combustion reaction which is quantified as a temperature ratio of the burned to unburned gas $\left(\mathrm{T}_{\mathrm{H}} / \mathrm{T}_{\mathrm{C}}\right)$. Each case used an identical mass flow rate of $0.35 \mathrm{~kg} / \mathrm{s}$ at a temperature of $422 \mathrm{~K}$. The static pressure of the domain's exit plane was held constant at $130 \mathrm{kPa}$ absolute which had minimal effect on the pressure at the inlet such that the inlet Mach number for all 5 cases was roughly constant and uniform at $M=0.12$. The laminar flame speed for each case was held constant at $0.6 \mathrm{~m} / \mathrm{s}$.

Table 3.1 - Temperature ratio parameter for each of the five simulations

\begin{tabular}{|c|c|c|}
\hline Case & $\mathbf{T}_{\mathbf{H}} / \mathbf{T}_{\mathbf{C}}$ & \% Heat Release \\
\hline $\mathrm{A}$ & 1.00 & 0 \\
\hline $\mathrm{B}$ & 1.75 & 25 \\
\hline $\mathrm{C}$ & 2.50 & 50 \\
\hline $\mathrm{D}$ & 3.25 & 100 \\
\hline $\mathrm{E}$ & 4.00 & 100 \\
\hline
\end{tabular}

\subsubsection{Simulation of Line-of-Sight Chemiluminescence Measurement}

Since the chemiluminescence signal is a line of sight measurement by nature, each pixel of the high-speed camera's sensor represents an integral quantity of chemiluminescence along its optical path. To account for this in the simulations, the cumulative heat release along simulated lines of sight were calculated. This was done in ANSYS Fluent by using a user-defined-scalar (UDS) which allows for the solution of a generic convection-diffusion partial differential equation for a generic scalar, $\varphi$, represented in equation 3.15 .

$$
\frac{\partial \varphi}{\partial t}+\frac{\partial \varphi}{\partial x_{i}}\left[F_{i} \varphi-D \frac{\partial \varphi}{\partial x_{i}}\right]=S_{\varphi}
$$

This equation was modified by setting the diffusion and unsteady terms to zero and then, by means of a set of user-defined-functions (UDFs), manipulated into the form shown in 
equation 3.16 which equates the growth rate of the passive scalar in the direction of the view vector to the local heat-release rate of the premixed combustion model.

$$
\frac{\partial \varphi}{\partial \vec{x}_{v i e w}}=\dot{c}
$$

By assuming that the simulated camera is far away the view vectors become parallel everywhere in the domain thus simplifying the analysis. In this case, the view vector simply points in the z-direction, i.e. the spanwise direction. At the side wall farthest from the "camera" the scalar is assigned a Dirichlet-type boundary condition equal to zero. Finally, the side wall nearest the camera's location is treated as the projection plane for the accumulated signal and is assigned a zero-slope Neumann-type boundary condition as shown in the exploded view in figure 3.5 .

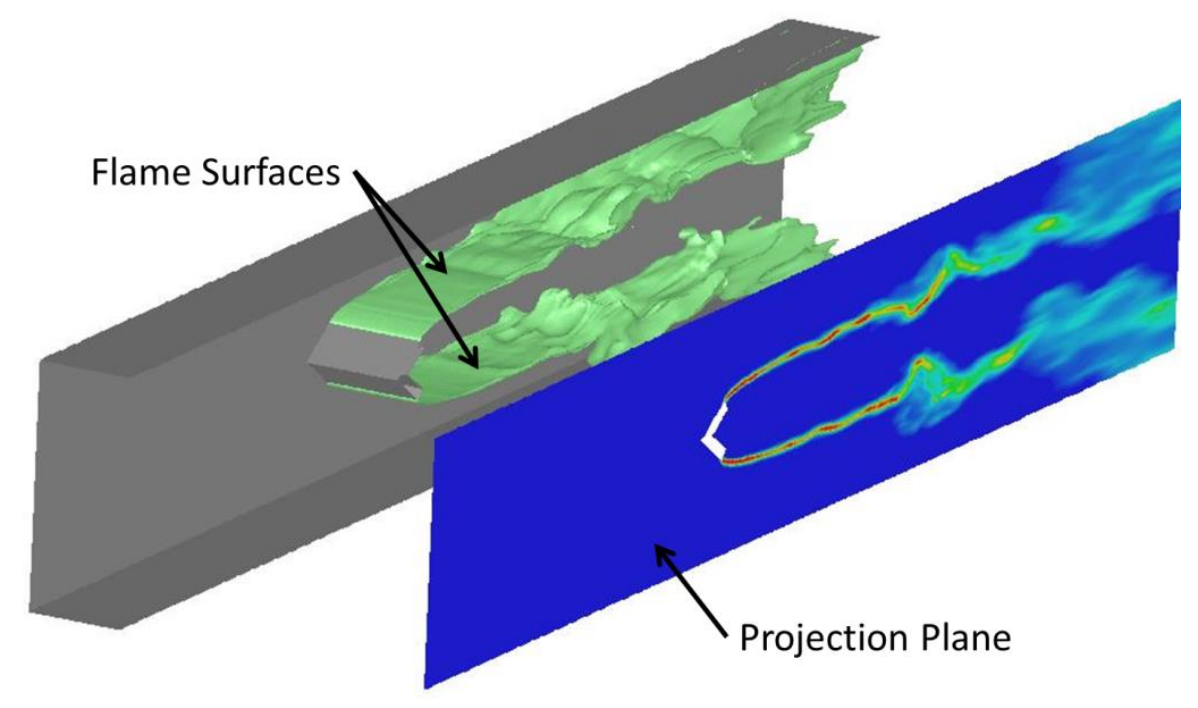

Figure 3.5 - Illustration of flame's heat release imaged to simulate the line-of-sight integrated chemiluminescence imaged in the experiment.

For the results reported here, the quantities of interest were sampled on a two 100x 100 grids of locations. Both grids have identical $x$ - and $y$-locations shown in the figure 3.6 , but the 
grid used for interrogating the velocity field was located half way between the two sidewalls. The grid used for interrogating the chemiluminescence field was located on the sidewall used as the projection plane. These quantities were sampled every 5 timesteps of the simulation.

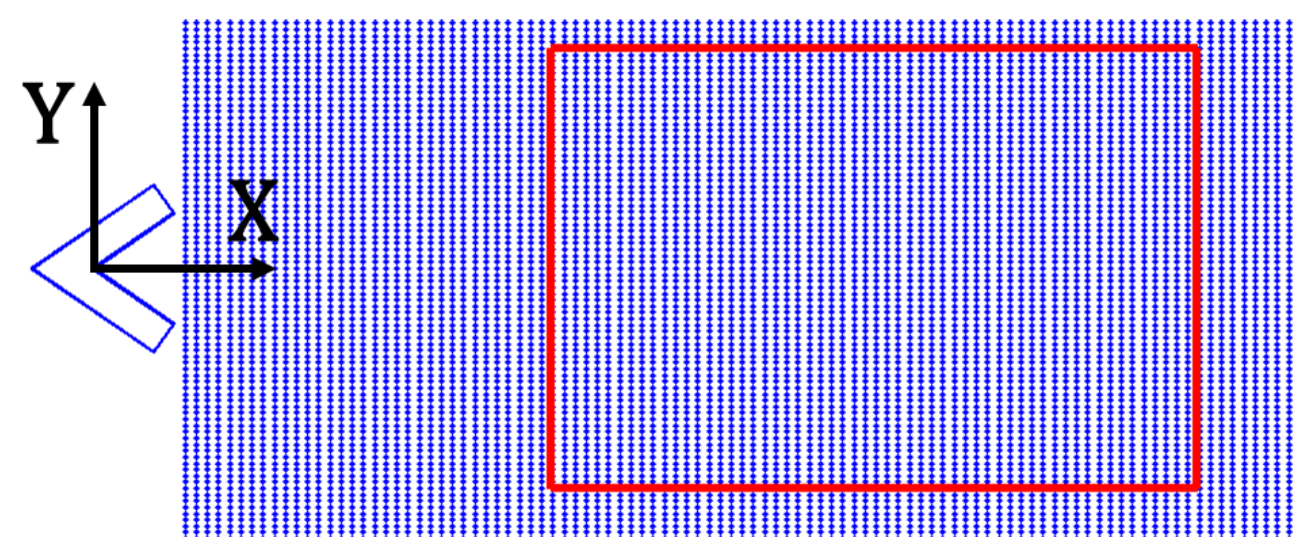

Figure 3.6 - X-and y-locations of sampling points used for interrogating the velocity and heat release quantities in the flow. Also shown are the locations of the vee-gutter flamholder and the side window relative to the sampling grid and coordinate axes.

\subsection{Results}

The two cases at the extreme ends of the study were validated against PIV measurements in the cold flow case and against chemiluminescence measurements in the reacting flow case. The time averages of the cold flow velocity measurements show good agreement with the timeaveraged LES data as shown in figure 3.7. 

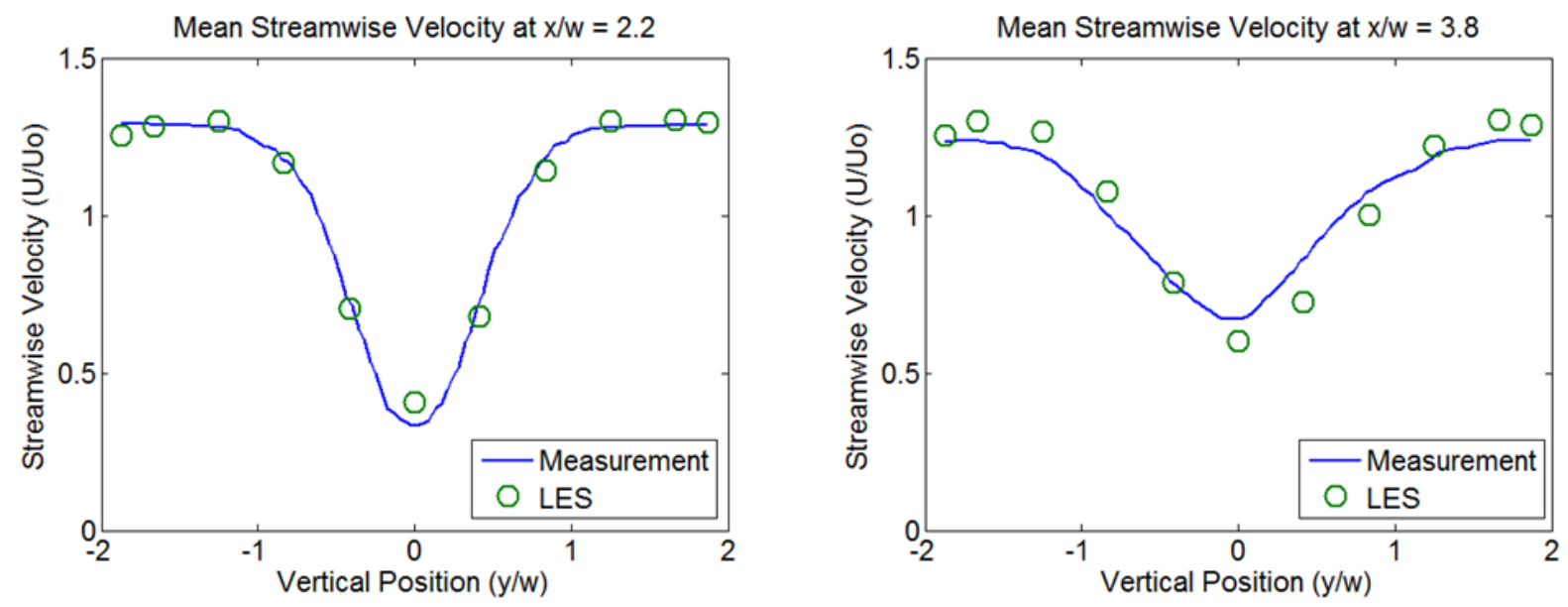

Figure 3.7 - Plot of time-averaged streamwise velocity profiles of the non-reacting condition at two locations downstream of the vee-gutter, $\mathrm{x} / \mathrm{w}=2.2$ (left) and $\mathrm{x} / \mathrm{w}=3.8$ (right).

The time-averaged chemiluminescence images show good agreement with the simulation in terms of the distance between the nearly parallel flame sheets, as shown in figure 8 . The experimental flame does show a somewhat thicker flamebrush than the simulated one as further shown in the plot of RMS contours in figure 3.9.
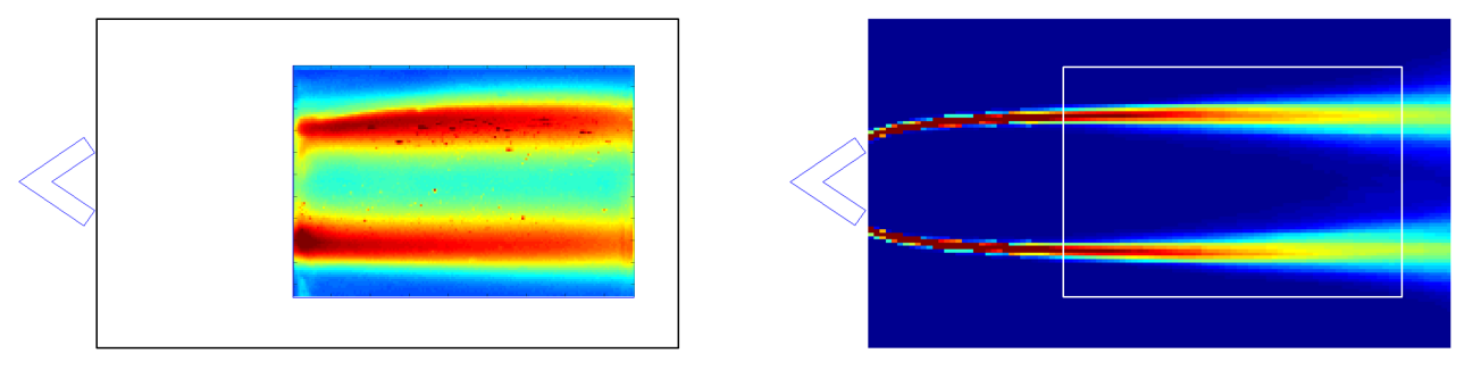

Figure 3.8 - Time-averaged chemiluminescence contours of the measured (left) and simulated (right) flames. Contours have arbitrary units. 

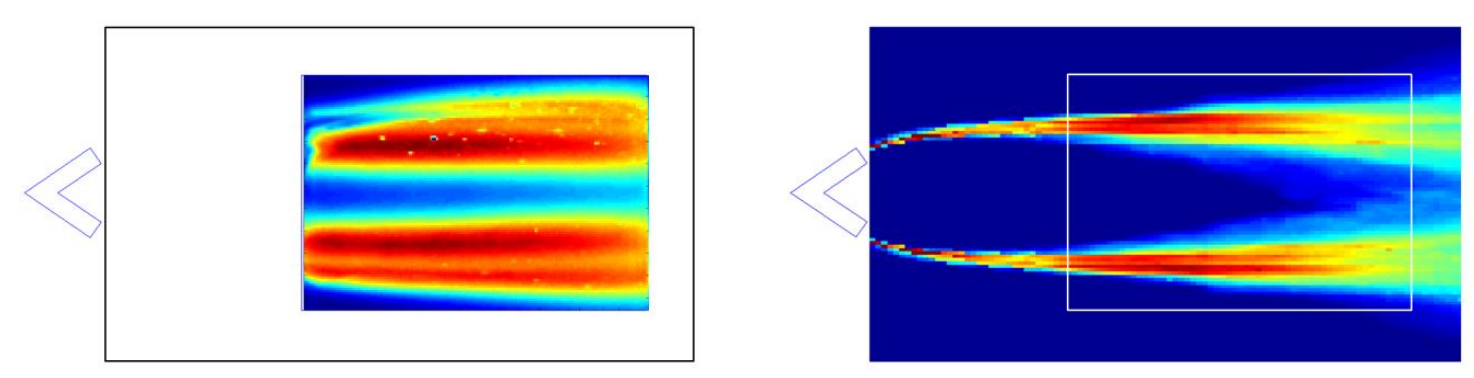

Figure 3.9 - RMS contours of chemiluminescence contours of the measured (left) and simulated (right) flames. Contours have arbitrary units.

These results indicated that, while not perfect, the simulations do capture the key components of the time-averaged flow field in the two cases able to be experimentally validated, namely the decay rate of the non-reacting wake and the near-zero flame angle observed in the fully-fueled case. Taken together these results give increased confidence in the models' abilities to accurately capture the relevant physics of the middle three cases $\left(\mathrm{T}_{\mathrm{H}} / \mathrm{T}_{\mathrm{C}}=1.75,2.5\right.$, and 3.25) and predict the points at which changes in the wake structures occur.

\subsubsection{Means Velocity Evolution with Temperature Ratio}

By plotting the contours of the time-average u-velocity fields, as shown in figure 3.10, it is clear that, holding all other model inputs constant, that the heat released by the combustion reaction acts to stabilize the wake, drastically reducing the decay rate. 


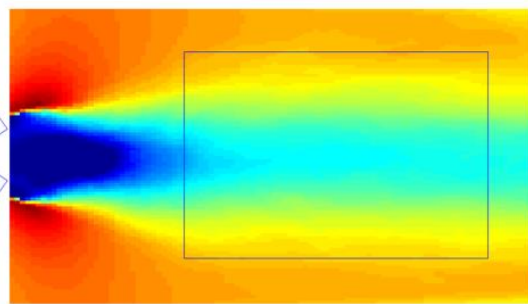

$\mathrm{Th} / \mathrm{Tc}=1.00$

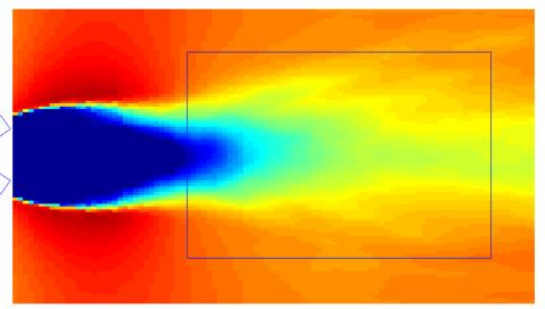

$\mathrm{Th} / \mathrm{Tc}=1.75$

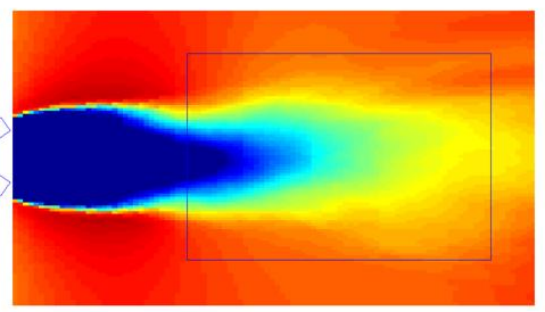

$\mathrm{Th} / \mathrm{Tc}=2.50$

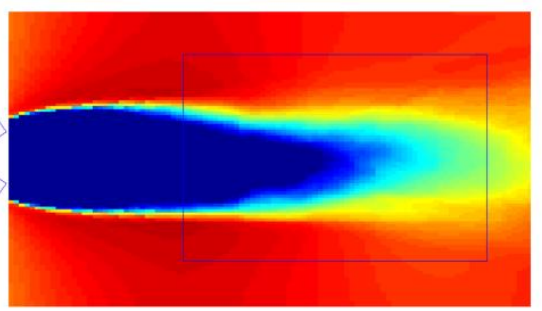

$\mathrm{Th} / \mathrm{Tc}=3.50$

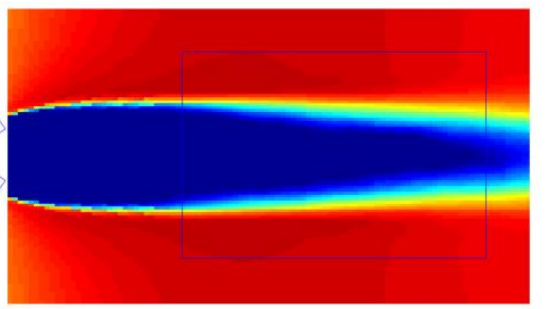

$\mathrm{Th} / \mathrm{Tc}=4.00$

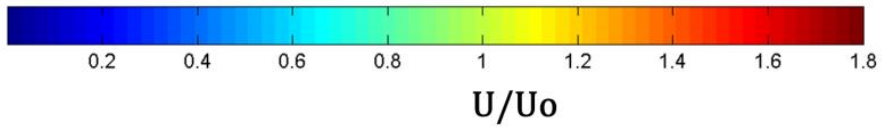

Figure 3.10 - Time-averaged u-velocity contours for the five conditions studied showing a clear pattern of wake-elongation with increasing heat release.

This trend is further illustrated by the time-averaged line of sight reaction rate integrals, shown in figure 3.11, that show two separate, near parallel flame sheets in the shear layers on the opposite edges of the vee-gutter extending downstream roughly as far as the recirculating wake 
reaches. At this point the flame sheets coalesce into a single broad brush which darts from the near-zero flame angle exhibited by the separate flamesheets, this trend persists up to the case with the highest heat release which never makes this transition.

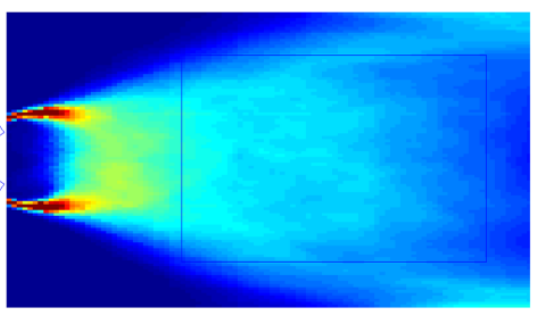

$\mathrm{Th} / \mathrm{Tc}=1.00$

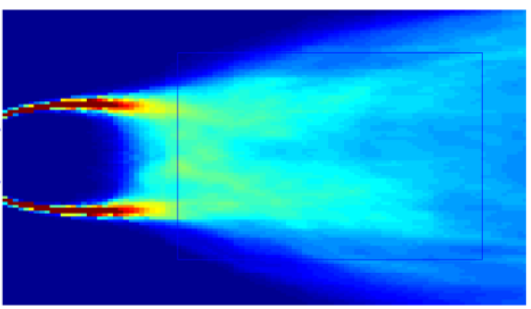

$\mathrm{Th} / \mathrm{Tc}=1.75$

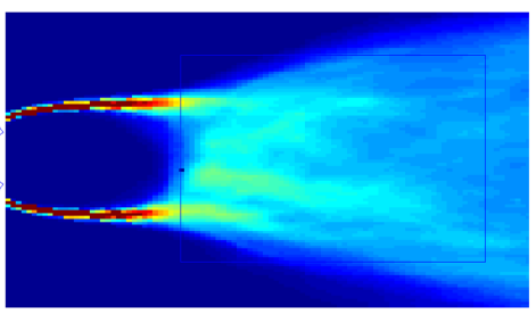

$\mathrm{Th} / \mathrm{Tc}=2.50$

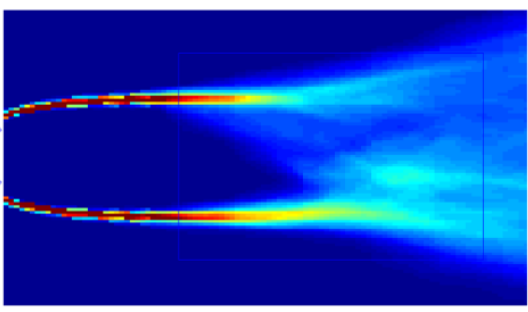

$\mathrm{Th} / \mathrm{Tc}=3.50$

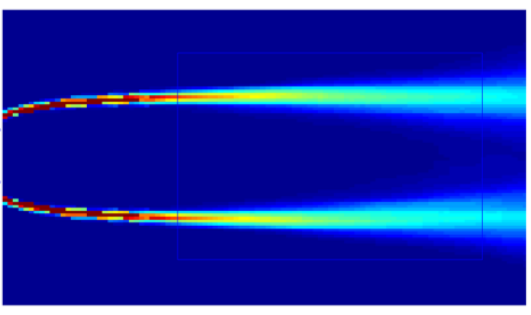

$\mathrm{Th} / \mathrm{Tc}=4.00$

Line of Sight Reaction Rate Integral (a.u.)

Figure 3.11 - Time-averaged line of sight reaction rate contours showing how the flame sheets' coalescence point moves farther downstream with increasing heat release until the two sheets finally remain separate permanently for the extreme case corresponding to the stoichiometric 
equivalence ratio.

\subsubsection{POD Results}

As was done with the time-average data of the extreme ends of the heat release spectrum, the dynamics of these cases could be compared via POD with experimental measurements. In the case of the non-reacting case, the $\mathrm{u}$ - and v-velocity contours of the first POD modes were compared against the same modes of the simulation. The results, normalized by the bulk velocity and shown in figure 3.12, show excellent agreement in both magnitude and spatial location, shape, and size.
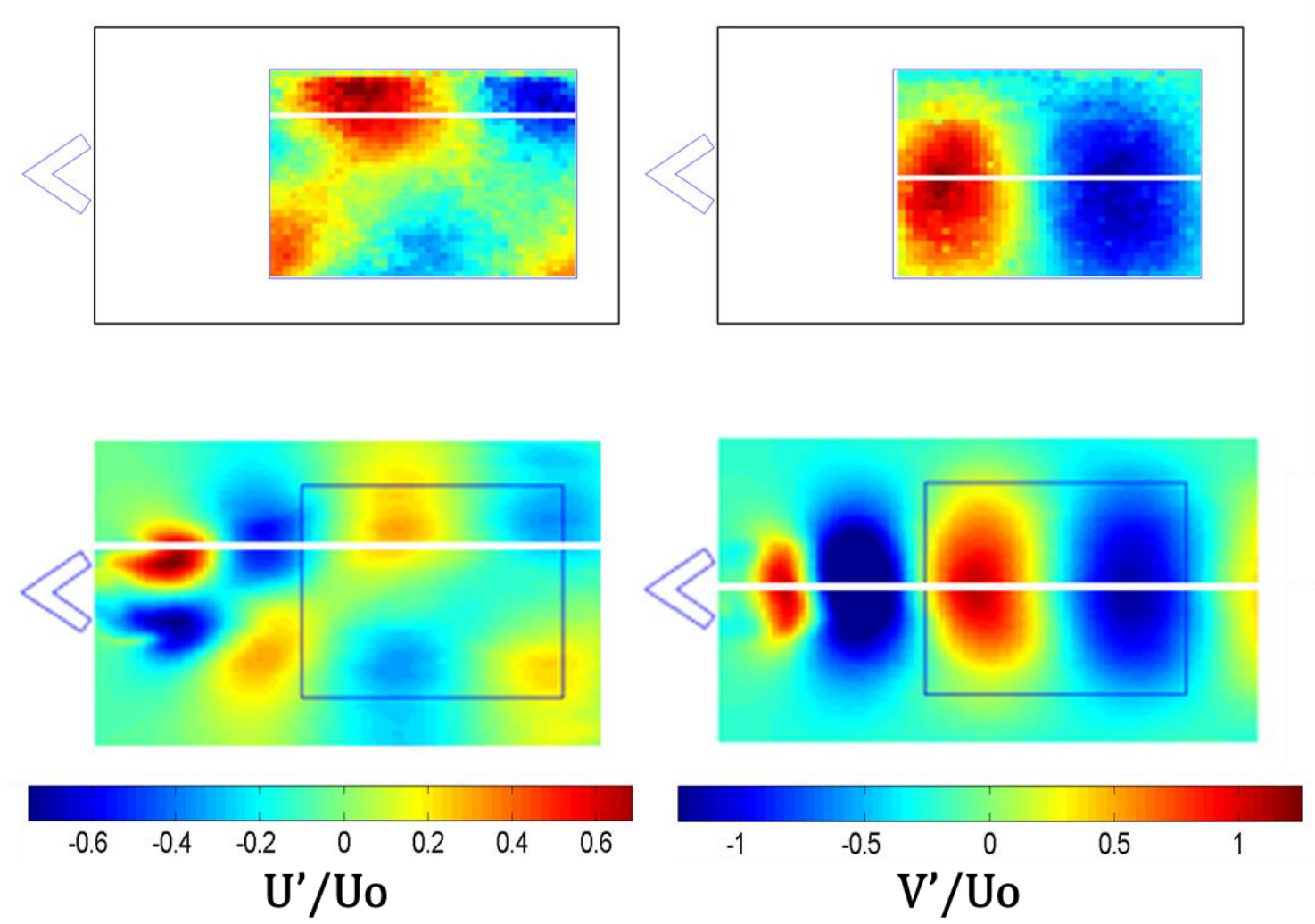

Figure 3.12 - Comparison of the first POD modes of u-velocity (left) and v-velocity (right) between the PIV measurements (top) and large eddy simulation (bottom)

At the other end of the spectrum, the reaction rate integrals of the combusting case showed good agreement with the length scales of the first POD mode between the simulated and 
experimental flame. Shown in figure 13, the discrepancy in flame sheet thicknesses observed the in time-averaged data persists to this mode, namely that the brush of the two flame sheets is thicker in the experimental flame than the one seen in the chemiluminescence measurements.
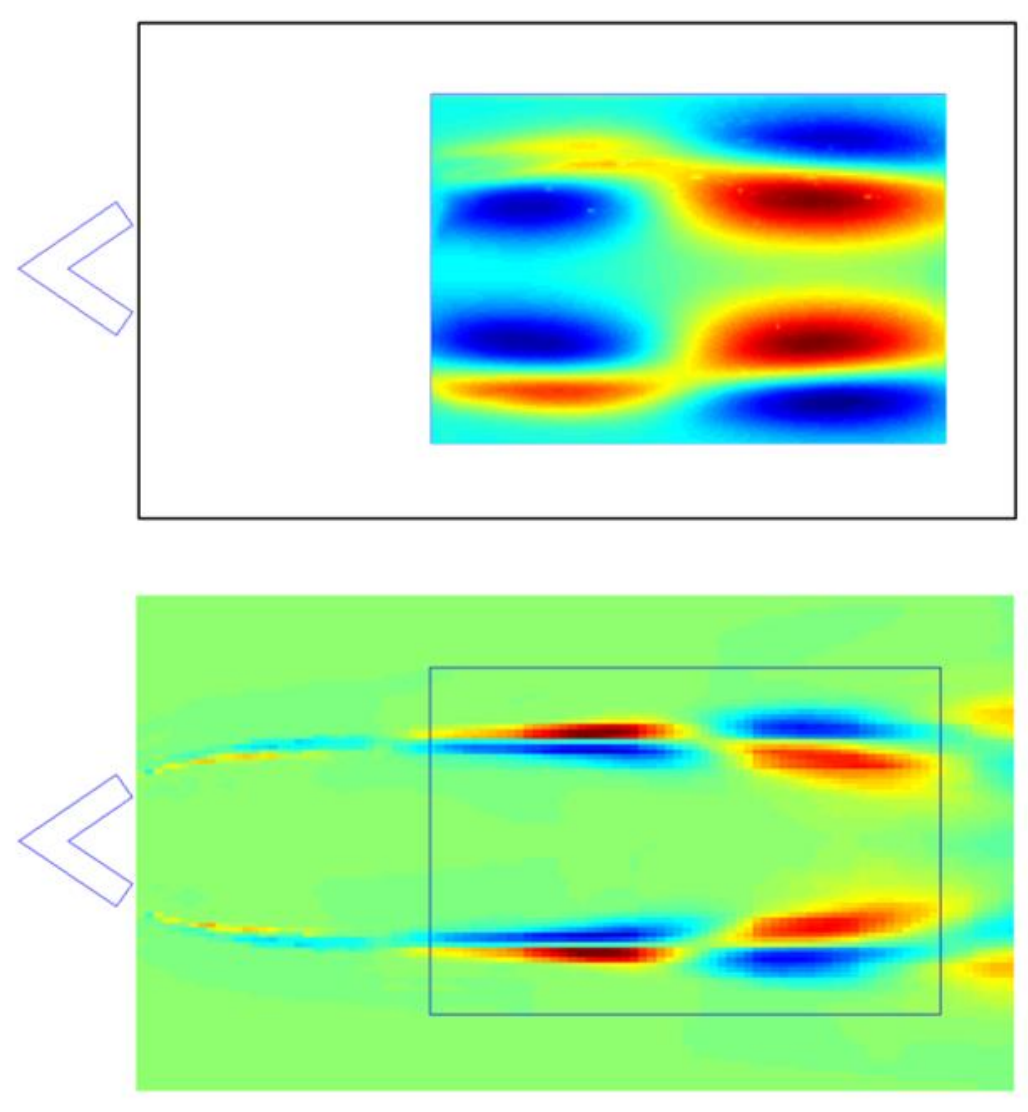

Figure 3.13 - Comparison between the first POD mode of line-of-sight chemiluminescence as measured in the experiment (top) and as simulated numerically (bottom).

Given the quality of the agreement of between the measurements and the simulations at the extreme ends of the heat release spectrum, it is reasonable to expect a similar level of accuracy for the intermediate cases which cannot be validated experimentally.

\subsubsection{First Modes of Velocity and Chemiluminescence}

The trends seen in the effect of heat release on the mean flow structures are repeated and extended in the POD analysis of the same data. First, in the case of the first POD mode of u- 
velocity (streamwise component) shown in figure 3.14, increased heat release corresponds to shifting the modeshapes farther and farther downstream while simultaneously reducing their intensity. Further the shape of the mode undergoes a significant change, namely that the asymmetric oscillations seen the in the 3 cases with the lowest heat release, become very skewed in the $T_{H} / T_{C}=3.25$ case and switch to a fully symmetric, and very weak structure in the case of full heat release. 


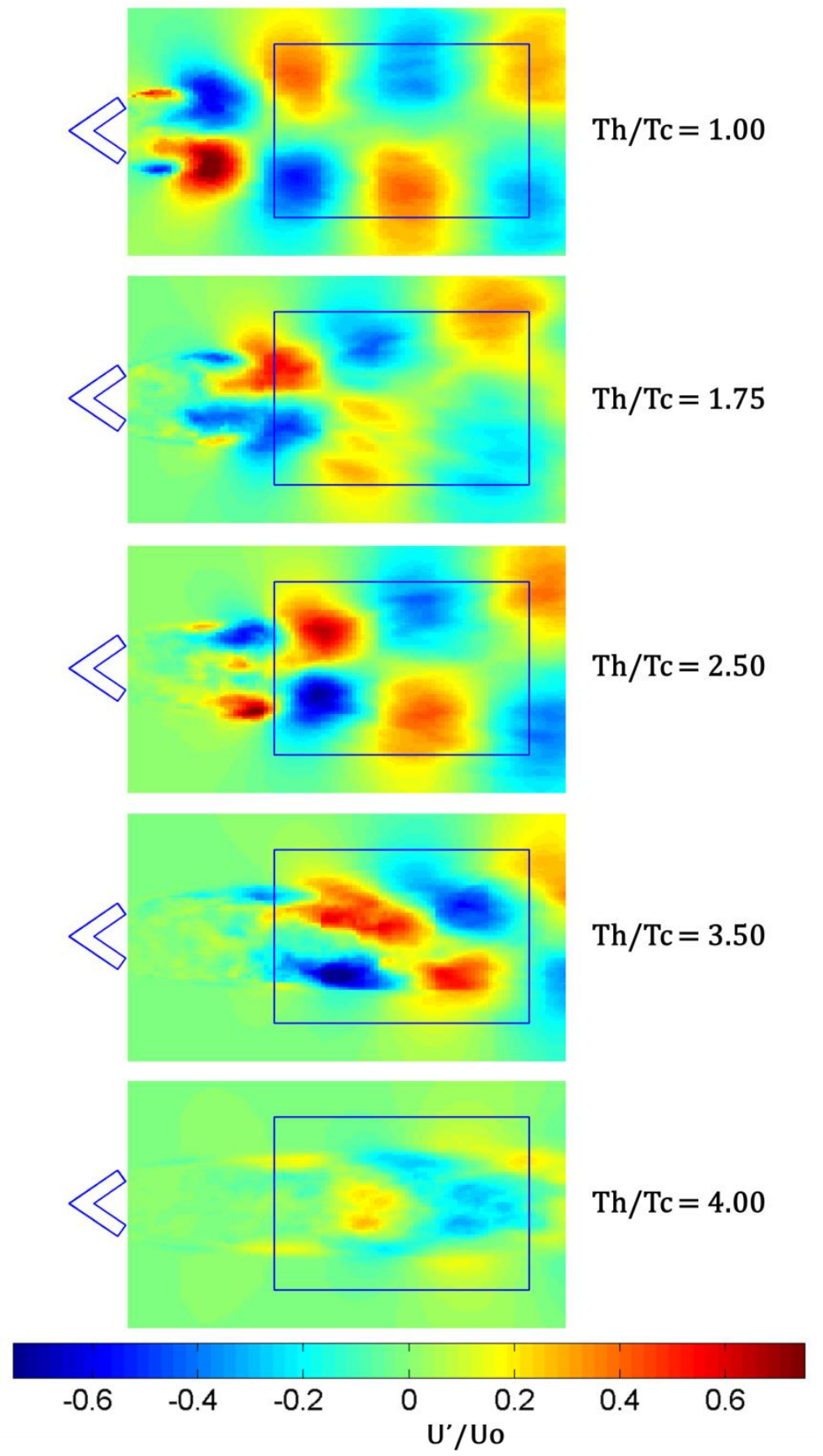

Figure 3.14 - U-velocity contours of the first POD mode normalized by the bulk velocity.

This result is further demonstrated by comparing the v-velocity components of the first POD modes of each case. Again, increasing heat release is associated with weakening oscillation patters and mode shapes that are shifted farther and farther downstream. The first three cases again look structurally very similar to one another just shifted farther downstream until case D 
which shows a stretching and skewing of the modes and finally in case E, a mode shape appears that is qualitatively different from any of the others and much weaker as well.

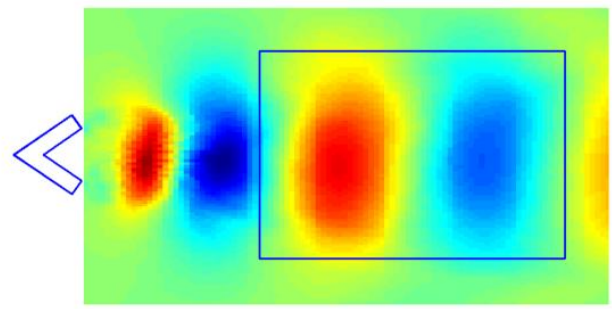

$\mathrm{Th} / \mathrm{Tc}=1.00$

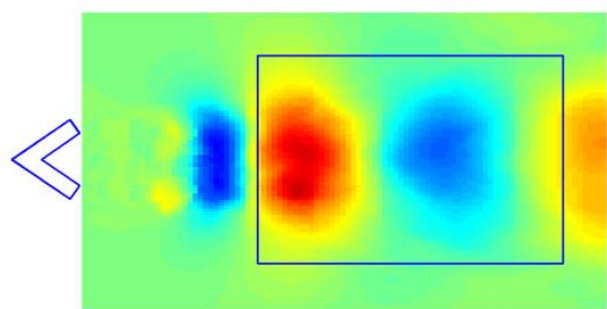

$\mathrm{Th} / \mathrm{Tc}=1.75$

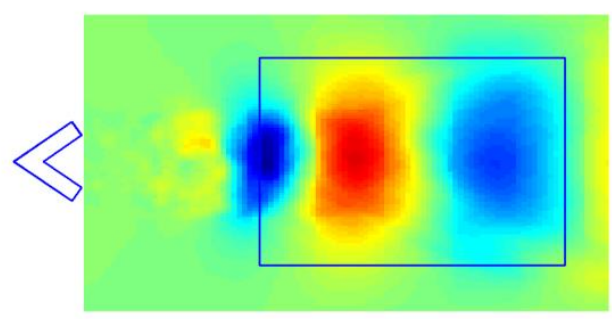

$\mathrm{Th} / \mathrm{Tc}=2.50$

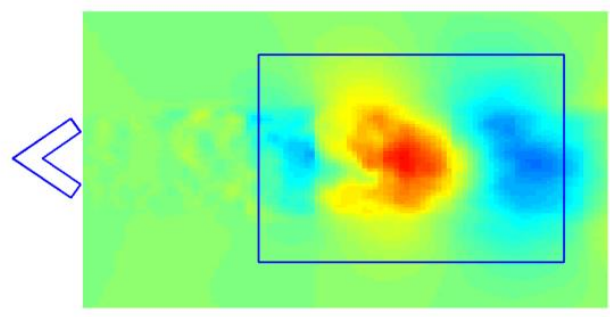

$\mathrm{Th} / \mathrm{Tc}=3.50$

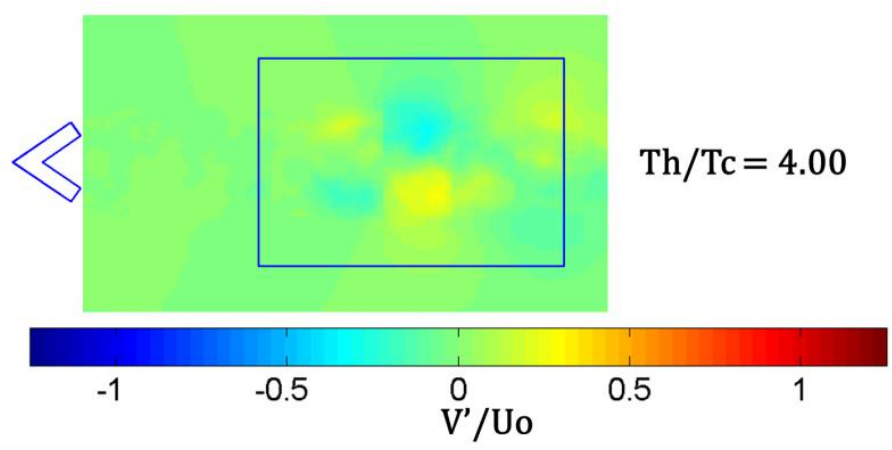

Figure 3.15 - V-velocity contours of the first POD mode normalized by the bulk velocity. 
The asymmetric to symmetric shift is more clearly demonstrated by examining the contours of the first POD mode of reaction rate line integrals shown in figure 3.16. Here, beginning in case $\mathrm{B}$, the oscillation mode is actually symmetric in the region starting at the veegutter and extending downstream as far as the recirculation zone stretches, at this point the reaction becomes asymmetric and widens as it flows downstream. This effect continues in cases $\mathrm{C}$ and $\mathrm{D}$ with the transition point shifting farther downstream. In case $\mathrm{D}$, the transition to completely symmetric oscillation begins to appear in the form of skewed modeshapes that are much more elongated and narrow than those seen in cases A-C. Finally in case E, the transition is complete and the oscillations reach the fully symmetric pattern seen the POD analysis of the experimental flame. 


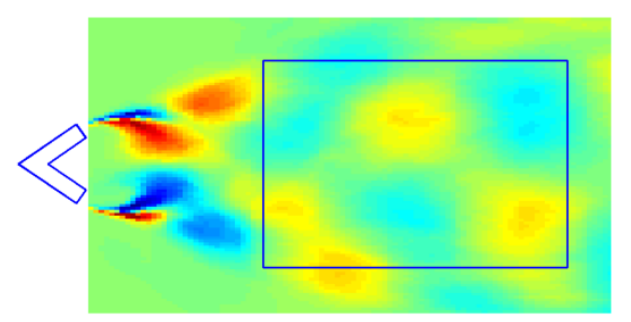

$\mathrm{Th} / \mathrm{Tc}=1.00$

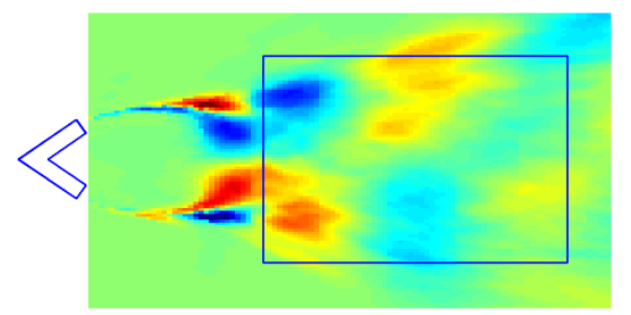

$\mathrm{Th} / \mathrm{Tc}=1.75$

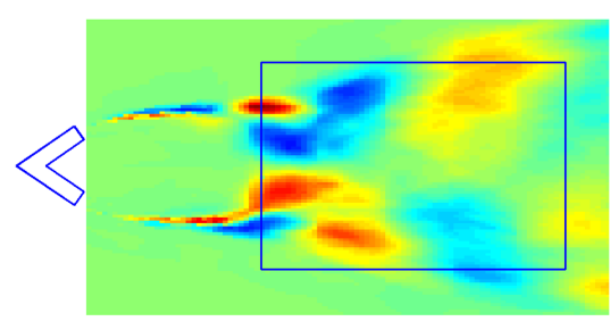

$\mathrm{Th} / \mathrm{Tc}=2.50$

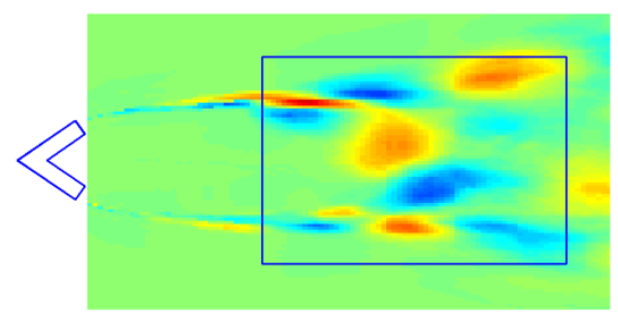

$\mathrm{Th} / \mathrm{Tc}=3.50$

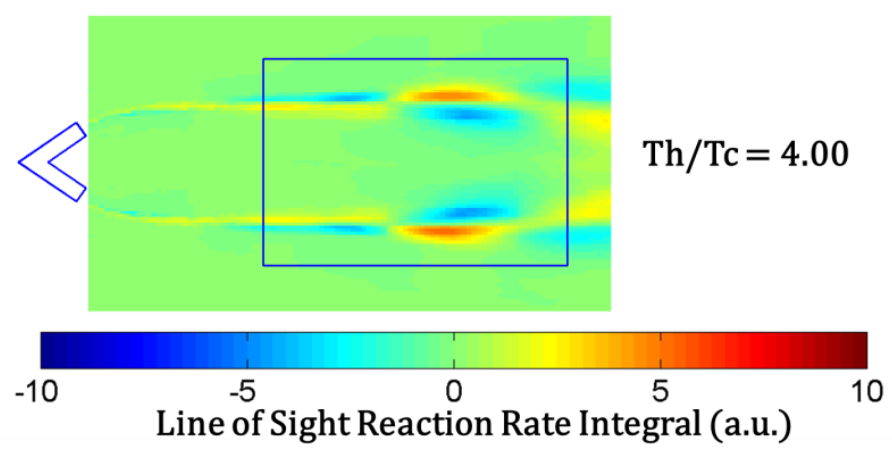

Figure 3.16 - Line of sight reaction rate integral contours of the first POD mode.

Further byproduct of the shift in flow structure can be seen from analyzing the frequency content of the coefficients of the first POD mode of each case to identify the fundamental frequency of oscillation. As shown in figure 3.17, cases A-C show a clear increase in the frequency of the first POD mode with increasing heat release, then when the transition between 
asymmetric and symmetric oscillations begins to occur in case $\mathrm{D}$, the trend is broken and essentially no further increase in frequency is seen. Finally, in case E the bubble bursts and the fundamental frequency actually starts to fall, further indicating that the nature of the wake's coherent structures has undergone a qualitative, not just quantitative transformation.

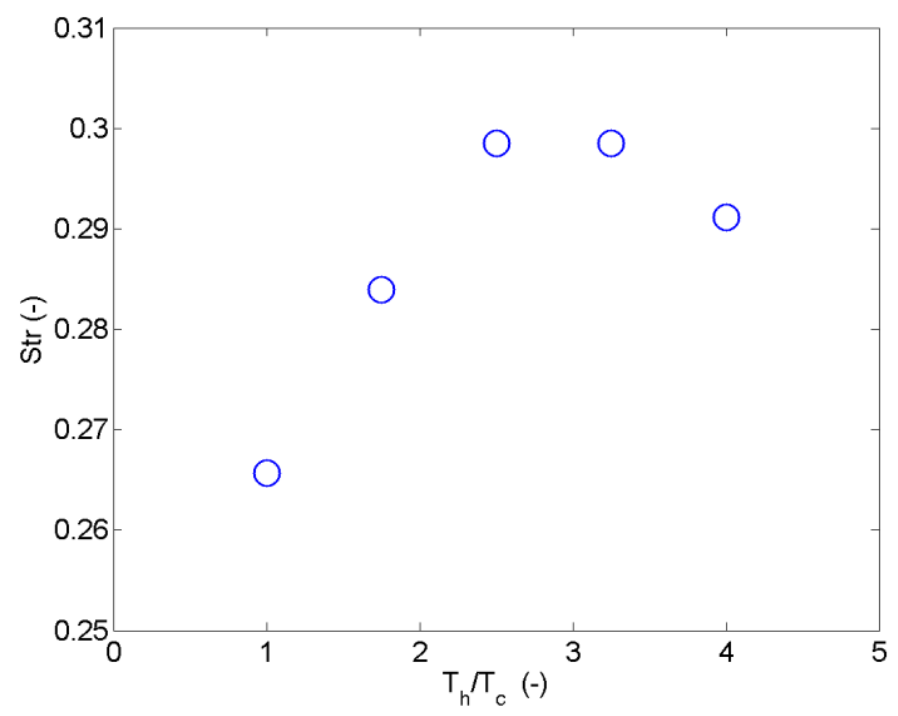

Figure 3.17 - Strouhal number of dominant shedding mode as a function of temperature ratio.

\subsection{Conclusion}

The results here show how the nature of the asymmetric, alternating shedding pattern familiar in non-reacting bluff-body wakes transitions to the symmetric, simultaneous shedding pattern observed experimentally in the reacting case of the same flow. With heat release below half of the nominal condition (Case E), the asymmetric structures shift farther downstream as the flame's heat release stabilizes the wake near the vee-gutter and extends the recirculation zone farther downstream behind the vee-gutter. Between half and full heat release however, the structure of the shedding begins a dramatic shift which causes the mode shapes to become skewed and the trend in fundamental frequency to change from upward to downward with increasing heat release. This result sheds new light on similar phenomena reported in the literature. For example, using POD to filter out the dominant modes of heat release illustrates with greater clarity than [7] how the most energetic mode of the flow evolves with temperature ratio. Further, these results show that the symmetry-asymmetry trends observed experimental by 
[20 - 21] can be explained in terms of variable temperature ratio while holding chemical and fluid timescales constant rather than through changes in the equivalence ratio. 


\subsection{Nomenclature}

\begin{tabular}{|c|c|}
\hline POD & Proper Orthogonal Decomposition \\
\hline Str & Strouhal number \\
\hline$f$ & frequency \\
\hline$w$ & Width of vee-gutter bluff body \\
\hline $\mathrm{U}$ & Bulk flow velocity \\
\hline $\operatorname{Re}$ & Reynolds number and similar abbreviations do not use italics \\
\hline$\rho$ & Density \\
\hline$\mu$ & Dynamic viscosity \\
\hline$A$ & Cross-sectional area \\
\hline Ro & Roschko Number \\
\hline Blockage & Blockage Ratio of Bluff Body \\
\hline RHS & Right Hand Side \\
\hline LHS & Left Hand Side \\
\hline$u$ & Snapshot vector of instantaneous data \\
\hline$u$ & Matrix of snapshot vectors \\
\hline$c$ & Correlation Matrix \\
\hline$\lambda$ & Vector of Eigenvalues \\
\hline & Eigenmode matrix \\
\hline
\end{tabular}


B

$u$

v

$P$

$e$

D

$R$

$T$

$\sigma$

$c_{v}$

V

C

$\Delta$

$\tau$

$k$

$S$

c

SGS
Eigenvector matrix

Velocity, x-direction

Velocity, y-direction

Pressure

Internal energy

Diffusivity

Gas constant

Temperature

Strain rate

Specific heat

Volume

Progress variable

Filter width

Stress

Kinetic energy

Flame Speed

Progress Variable

Subgrid scale 


\subsection{References}

[1] Blanchard, R., Ng, W., Lowe, K., and Vandsburger, U., (2014) "Simulating Bluff Body Flameholders: On the Use of Proper Orthogonal Decomposition for Wake Dynamics Validation.” ASME Journal of Engineering for Gas Turbines and Power.

[2] Blanchard, R., Wickersham, A.J., Ma, L., and Ng, W., (2014) "Simulating Bluff-Body Flameholders: On the Use of Proper Orthogonal Decomposition for Combustion Dynamics Validation.” ASME Journal of Engineering for Gas Turbines and Power.

[3] Macquisten, M., and Dowling, A., (1993) "Low Frequency Combustion Oscillations in a Model Afterburner." Combustion and Flame, 94 pp. 253-264.

[4] Giacomazzi, E., Battaglia, V., and Bruno, C. (2004). "The coupling of turbulence and chemistry in a premixed bluff-body flame as studied by LES." Combustion and Flame, 138 (4) pp. 320-335. doi:10.1016/j.combustflame.2004.06.004

[5] Shanbhogue, S. J., Husain, S., and Lieuwen, T. (2009). "Lean blowoff of bluff body stabilized flames: Scaling and dynamics" Progress in Energy and Combustion Science 35 (1) pp. 98-120. doi:10.1016/j.pecs.2008.07.003

[6] Cocks, P., Sankaran, V., and Soteriou, M., (2013) "Is LES of Reacting Flows Predictive? Part 1 : Impact of Numerics" 51 $1^{\text {st }}$ AIAA Aerospace Sciences Meeting 2013, pp 1-29.

[7] Giezendanner, R., Keck, O., Weigand, P., Meier, W., Meier, U., Stricker, W., and Aigner, M., (2003) "Periodic combustion instabilities in a swirl burner studied by phase-locked planar laser-induced fluorescence" Combustion Science and Technology, 175 (4) pp 721-741. doi:10.1080/00102200302390 
[8] Pearson, K. (1901). On lines and Planes of Closest Fit to Systems of Points in Space.

Philosophical Magazine, 2 pp. 559-572.

[9] Lumley, J., (1967) “The Structure of Inhomogeneous Turbulent Flows,” Atmospheric Turbulence and Radio Wave Propagation, 114 pp. 166-178.

[10] Gergen, I., and Harmanescu, M. (2012), “Application of principal component analysis in the pollution assessment with heavy metals of vegetable food chain in the old mining areas" Chemistry Central Journal, 6 (1) pp 156. doi:10.1186/1752-153X-6-156

[11] Smith, T., Moehlis, J., and Holmes, P., (2005) "Low-Dimensional Modelling of Turbulence Using the Proper Orthogonal Decomposition: A Tutorial,” Nonlinear Dynamics, 41 (1-3) pp. 275-307.

[12] Sirovich, L., and Kirby, M., (1987) "Low-dimensional procedure for the characterization of human faces," Journal of the Optical Society of America, 4 (3) pp. 519.

[13] Hsieh, T., Chen, J., Chen, L., Chiang, P., and Lee, H., (2011) “Time-course gait analysis of hemiparkinsonian rats following 6-hydroxydopamine lesion.," Behavioral Brain Research, 222 (1) pp. 1-9.

[14] Berkooz, Gal, Holmes, P., and Lumley, J., (1993) “The Proper Orthogonal Decomposition in the Analysis of Turbulent Flows.” Annual Review of Fluid Mechanics 25, no. (1) pp. 539-575. doi:10.1146/annurev.fl.25.010193.002543.

[15] Fukunaga, K. (1990). Introduction to statistical pattern recognition. Academic press.p. 592.

[16] Moin, P., and Moser, R., (2006) "Characteristic-eddy decomposition of turbulence in a channel," Journal of Fluid Mechanics 200 pp. 471. 
[17] Sirovich, L., and Kirby, M., (1987) "Low-dimensional procedure for the characterization of human faces," Journal of the Optical Society of America, 4 (3) pp. 519.

[18] Duwig, C., and Iudiciani, P., (2009) "Extended Proper Orthogonal Decomposition for Analysis of Unsteady Flames," Flow, Turbulence, Combustion, 84 (1) pp. 25-47.

[19] Böhm, B., Brübach, J., Ertem, C., and Dreizler, A., "Experiments for Combustion-LES Validation," Flow, Turbulence, Combustion, 80 (4) pp. 507-529.

[20] Kostka, S., Roy, S., Huelskamp, B., Lynch, A., Kiel, B., and Gord, J. (2011)

“Characterization of Bluff-Body-Flame Vortex Shedding Using Proper Orthogonal

Decomposition" 49th AIAA Aerospace Sciences Meeting and Exhibit" pp 1-7.

[21] Kostka, S., Roy, S., Huelskamp, B., Lynch, A., Kiel, B., and Gord, J., (2012)

"Characterization of Flame-Shedding Behavior behind a Bluff-Body Using Proper Orthogonal Decomposition.” Combustion and Flame 159 (9) pp 2872-2882.

doi:10.1016/j.combustflame.2012.03.021.

[22] Anae, R., (1999) "Race, authority, and college football: The division of labor among college coaches” Ph.D. thesis, Sociology, Brigham Young University.

[23] Haber, L.C., (2000) "An Investigation into the Origin, Measurement and Application of Chemiluminescent Light Emissions from Premixed Flames” M.S. thesis, Mechanical Engineering, Virginia Tech.

[24] Farina, J., (2013) “Application of Multi-Port Mixing for Passive Suppression of ThermoAcoustic Instabilities in Premixed Combustors" Ph.D. thesis, Mechanical Engineering, Virginia Tech. 
[25] Wickersham, A.J., Xuesong L., and Ma, L., (2014) “Comparison of Fourier, Principal Component and Wavelet Analyses for High Speed Flame Measurements.” Computer Physics Communications 185, (4) pp. 1237-1245. doi:10.1016/j.cpc.2013.12.022.

[26] Smagornisky, J., (1963) "General Circulation Experiments with the Primitive Equations," Mon. Weather Rev., 91 (3) pp. 99-164.

[27] Germano, M., Piomelli, U., Moin, p., and Cabot, W., (1991) “A dynamic subgrid-scale eddy viscosity model," Physics of Fluids and Fluid Dynamics., 3 (7) pp. 1760.

[28] Lilly, D., (1992) “A proposed modification of the Germano subgrid-scale closure method," Physics of Fluids and Fluid Dynamics., 4 (3) pp. 633.

[29] Butler, T., and O’Rourke, P., (1977) “A Numerical Method for Two Dimensional Unsteady Reacting Flows.” Symposium (International) on Combustion, 16 (1) pp. 1503-1515. doi:10.1016/S0082-0784(77)80432-3.

[30] Selle, L., (2004) "Compressible Large Eddy Simulation of Turbulent Combustion in Complex Geometry on Unstructured Meshes.” Combustion and Flame, 137 (4) pp. 489-505. doi:10.1016/j.combustflame.2004.03.008.

[31] Poinsot, T., and Veynante, D., (2009) Theoretical and Numerical Combustion, RT Edwards.

[32] Huang, Ying, and Vigor Yang. "Dynamics and Stability of Lean-Premixed SwirlStabilized Combustion.” Progress in Energy and Combustion Science, 35 (4) pp. 293-364. doi:10.1016/j.pecs.2009.01.002.

[33] Zimont, V., Polifke, W., Bettelini, M., and Weisenstein, W., (1998) “An Efficient Computational Model for Premixed Turbulent Combustion at High Reynolds Numbers Based on 
a Turbulent Flame Speed Closure" Journal of Engineering for Gas Turbines and Power, 120 pp. $526-532$

[34] Zimont, V., Biagioli, F., and Syed, K., (2001) "Modelling Turbulent Premixed

Combustion in the Intermediate Steady Propagation Regime." Progress in Computational Fluid

Dynamics, 1 (1) pp. 14-28

[35] Lamoureux, N., Djebaïli-Chaumeix, N., and Paillard, C., (2003) "Laminar Flame

Velocity Determination for H2-air-He-CO2 Mixtures Using the Spherical Bomb Method.”

Experimental Thermal and Fluid Science, 27 (4) pp. 385-393. doi:10.1016/S0894-

1777(02)00243-1.

\section{Conclusion and Future Work}

The results shown in these papers demonstrate the value of the proposed technique for using POD as a validation tool for comparing time-accurate simulations with experimental data or other simulations. Advances in experimental instrumentation and diagnostic techniques have contributed to many significant advances in the type and quality of measurements that can be made in combustion research. Combustion modeling too has developed many new promising models and time-accurate simulation techniques to exploit the ever-expanding computational power available to researchers. This work provides a valuable approach for taking advantage of these developments more fully in order to determine which dynamic modes of an experimental system's behavior can be reproduced using numerical models of the same.

The papers demonstrate the use of this technique by applying it to large eddy simulations of the bluff body stabilized premixed combustion experiment. The results show where the models do and perhaps of equal importance, where the models don't match the dynamic modes of the experiment. By isolating the dynamic modes from each other via the proper orthogonal decomposition, it was shown for example in the second paper that the model was able to accurately reproduce certain low energy modes which exist the experiment, but which are so small that they are dwarfed by the other flow features. Knowing that the model has been 
validated for the case where these modes are small can give confidence that the same mode could be accurately predicted for a future application where its dynamics may not be so benign.

The sensitivity studies demonstrate that the technique can be equally useful in determining how the dynamics of two simulations differ, whether from two different codes or from the same code with different simulation parameters or boundary conditions. The first paper in particular showed that while different simulations showed widely differing magnitudes in the dynamics immediately behind the vee-gutter, these differences quickly vanished as the flow moved downstream. The POD-reduced data clearly showed how the downstream dynamics of the shed vortices were not simply artifacts of the flow conditions upstream.

A significant, if somewhat tangential contribution was made at the end of the first paper where the issue of POD convergence is treated in a practical way. First, it was shown that the eigenvalues and eigenmodes converge at different rates, second-order and first-order respectively. Since the technique used throughout this work depends heavily on proper orthogonal decomposition and since these operations require the manipulation of large matrices, the computational costs of performing the decomposition are not trivial. Solving the eigenvalue problem on the correlation matrix in particular requires significant time and memory to complete, typically taking $\mathrm{O}(\mathrm{N} 3)$ operations to complete. Thus, as the size of data sets increases the computational costs can quickly become prohibitive and a natural question arises as to how many snapshots are needed to represent a "converged" mode. Since the results show that the eigenvalues themselves shown 2nd-order convergence behavior and the eigenmodes obey a firstorder convergence rate, the answer to the question appears to be that as long as the timeaveraged data is converged, the POD modes and eigenvalues will be as well.

Finally, in the third paper, the method shows how the nature of the asymmetric, alternating shedding pattern familiar in non-reacting bluff-body wakes transitions to the symmetric, simultaneous shedding pattern observed experimentally in the reacting case of the same flow. The results show that for heat release below half of the nominal condition, the structures resemble the cold-flow condition from the first paper but with features that are simply shifted farther downstream. However using the POD to isolate the shedding mode from the other modes, it becomes clear that between half and full heat release, the structure of the shedding undergoes a dramatic shift which causes the mode shapes to become skewed and eventually flip to being fully symmetric. 
Future applications of this technique may also extend it into three-dimensional POD reconstructions of the entire LES domain. This may be done by sampling the flow at a number of points similar to that shown in this work but then using the extended POD technique used in the first paper to calculate any single mode of interest by using the eigenvalues of the given mode to calculate the weighted sum of the instantaneous snapshots of the full domain that are saved throughout the simulation at the same points the subset of points is sampled. For threedimensional simulations consisting of millions of cells with multiple variables of interest per cell, a full POD of hundreds or thousands of snapshots will likely always be infeasible, but by using extended POD, the recovery of a small number of modes may be possible over the entire domain.

Work is currently ongoing to couple this technique with tomographic chemiluminescence investigates of a partially premixed flames. In this case the chemiluminescence signals will be integrated along multiple lines of sight and can be used not only as a three-dimensional validation of the flame but could also serve as a testbed for developing techniques for calculating tomographic inverses.. 


\section{A APPENDIX: Rig Drawings}

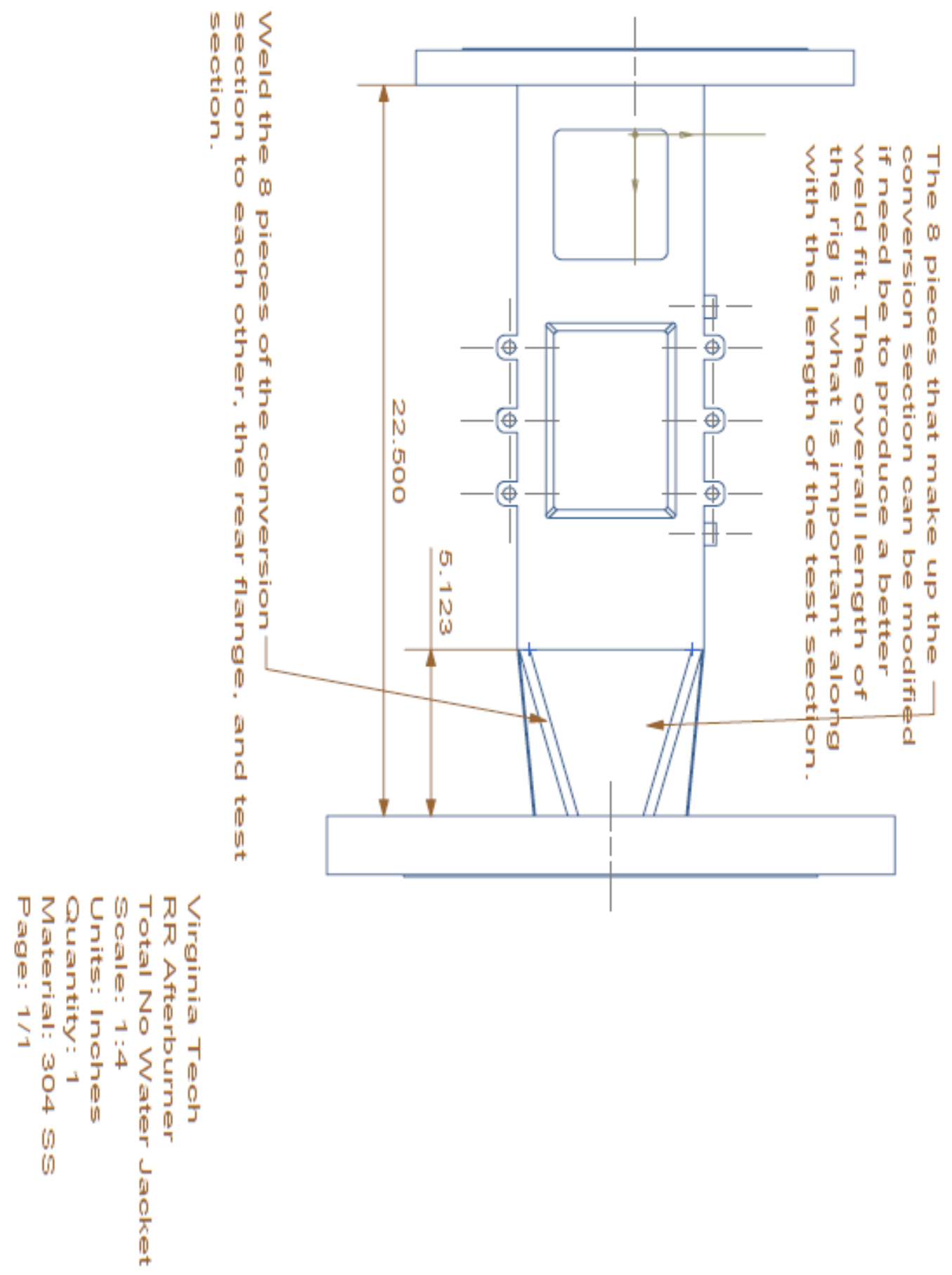




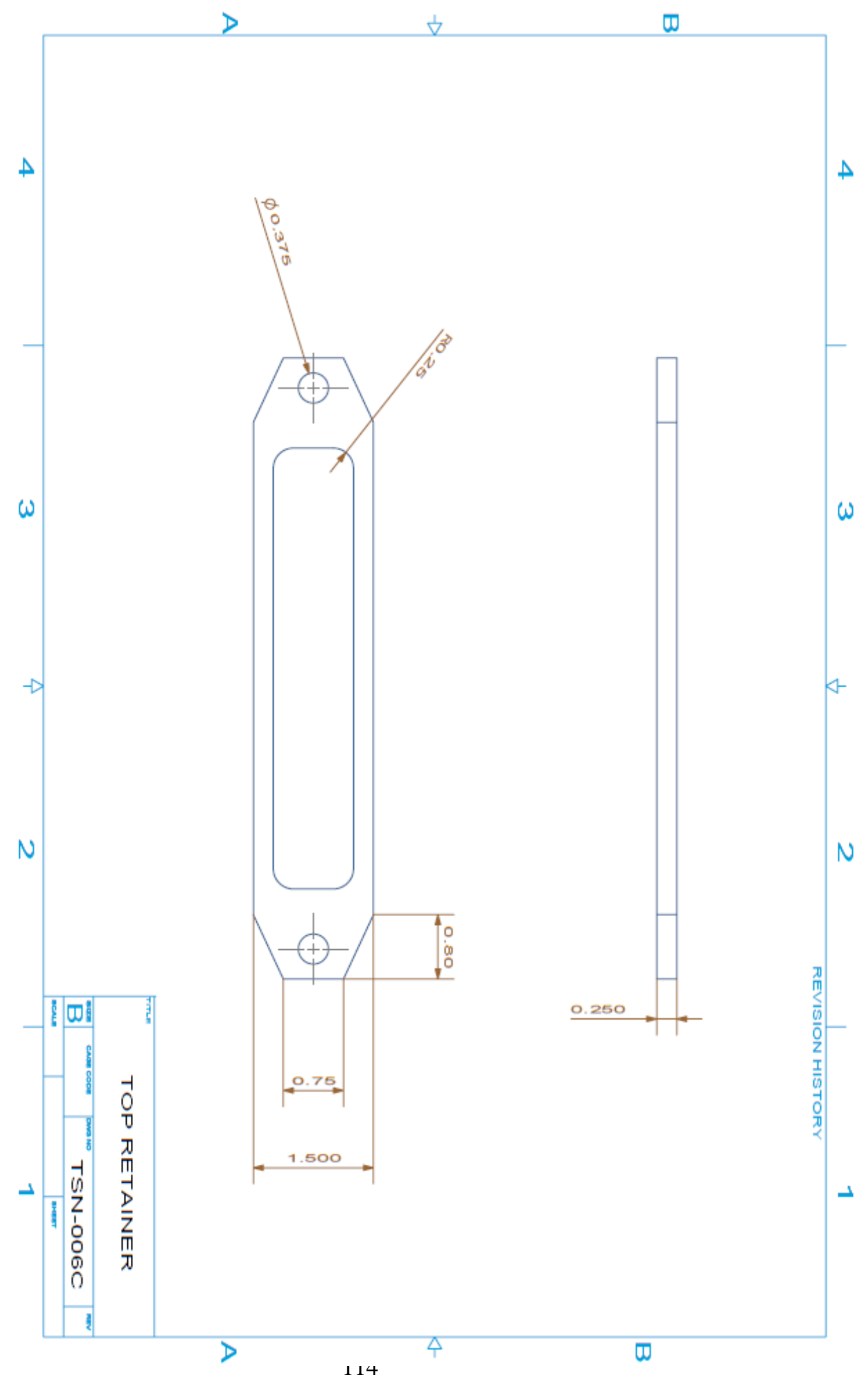




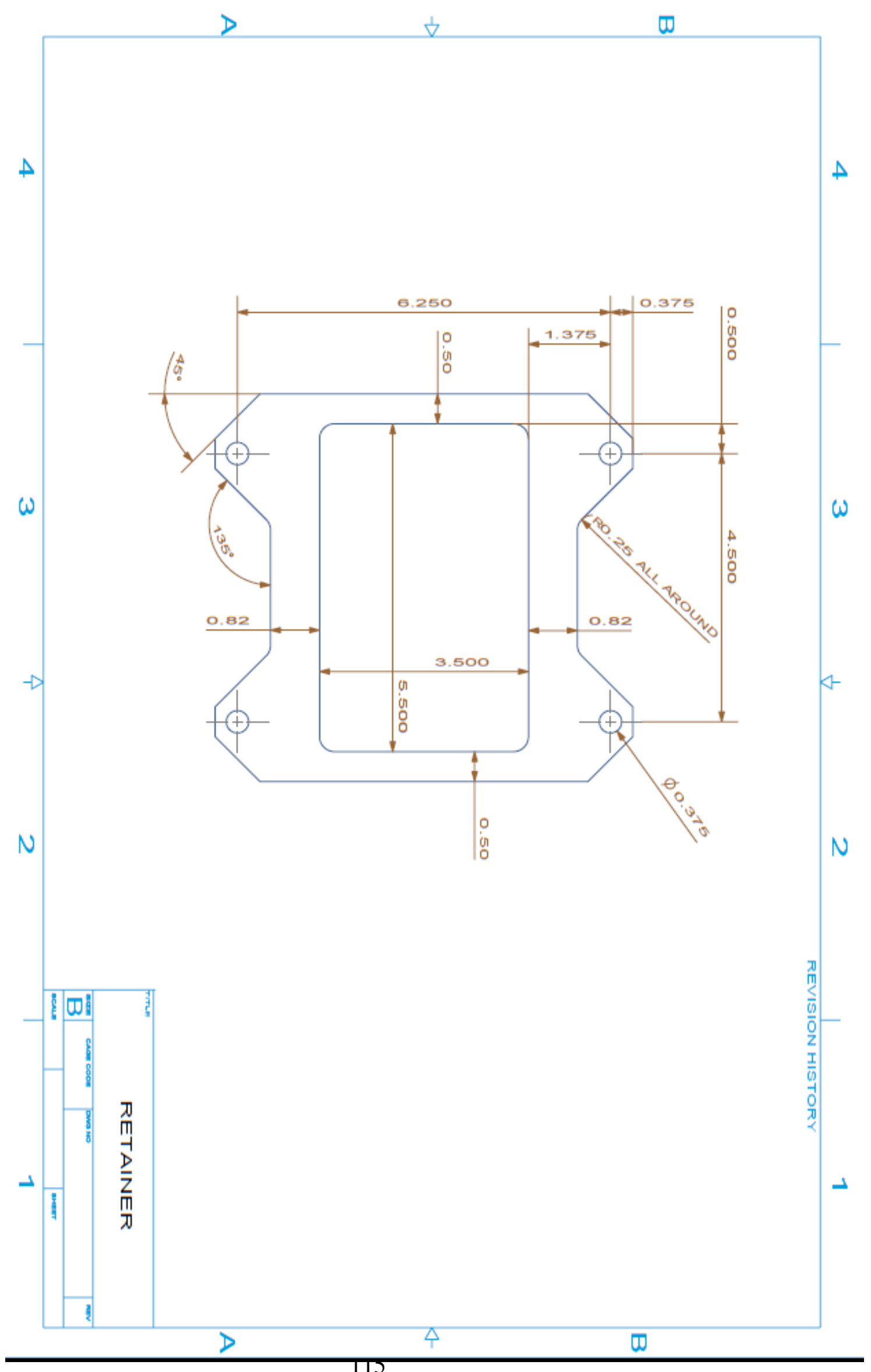




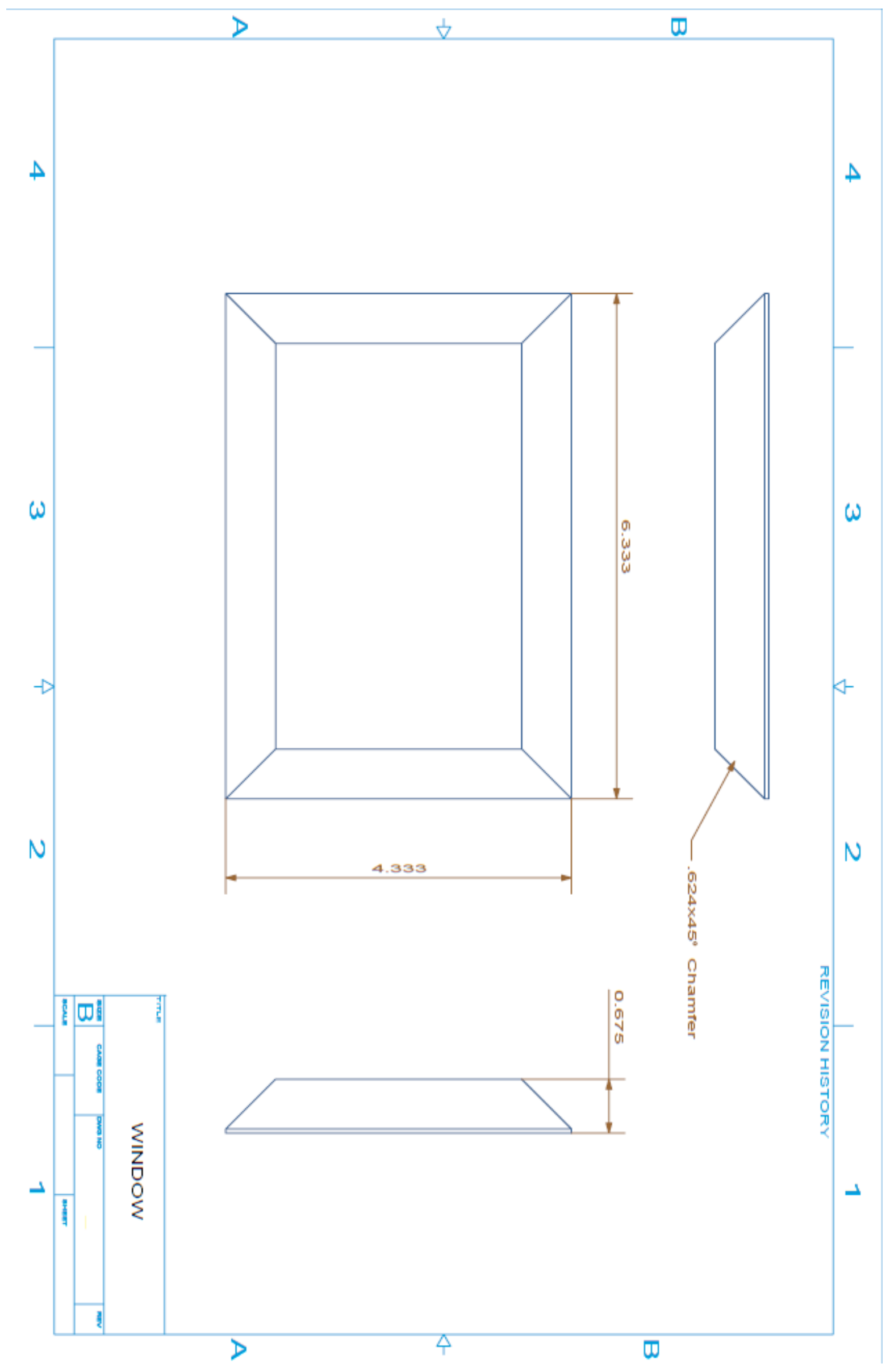




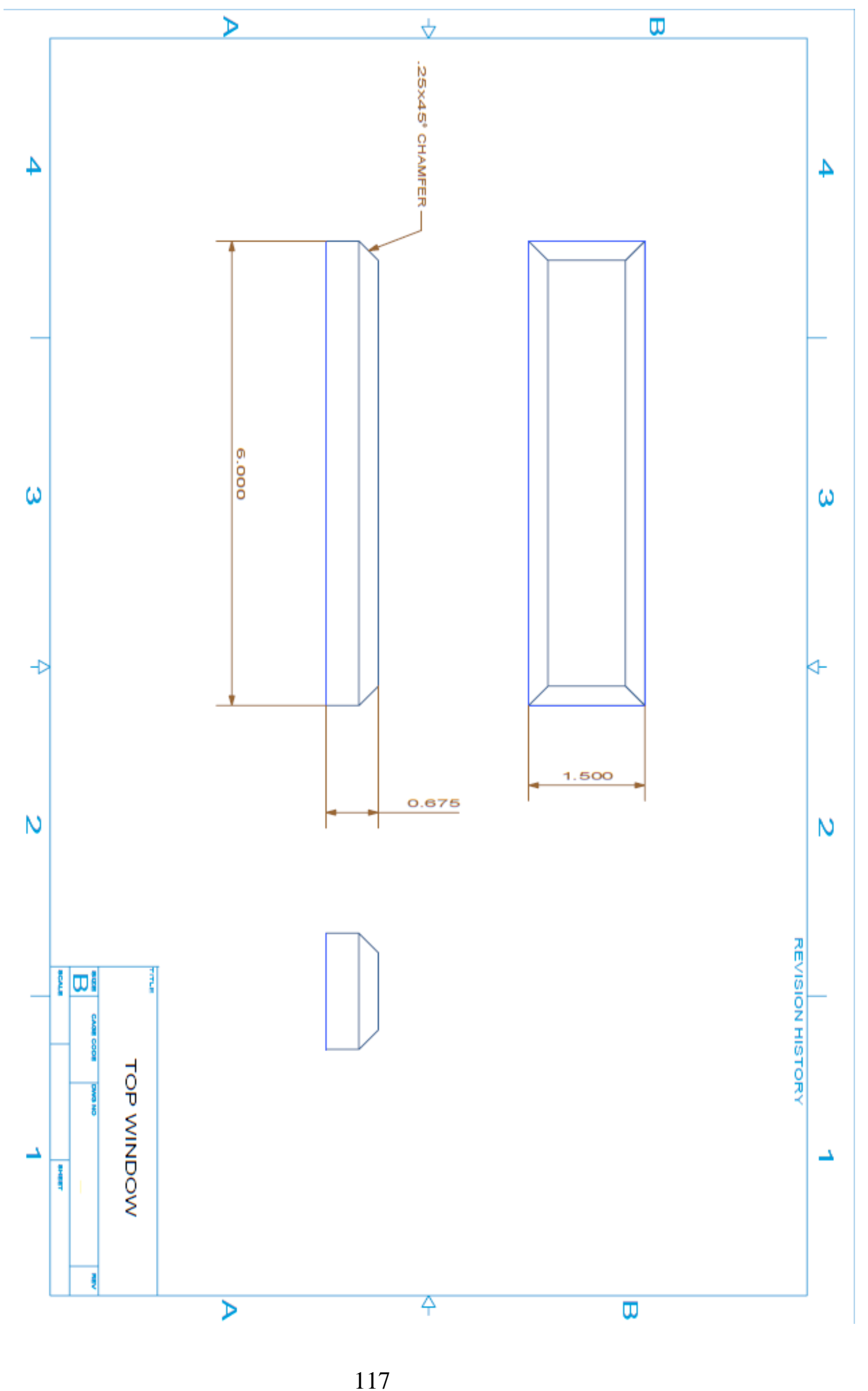




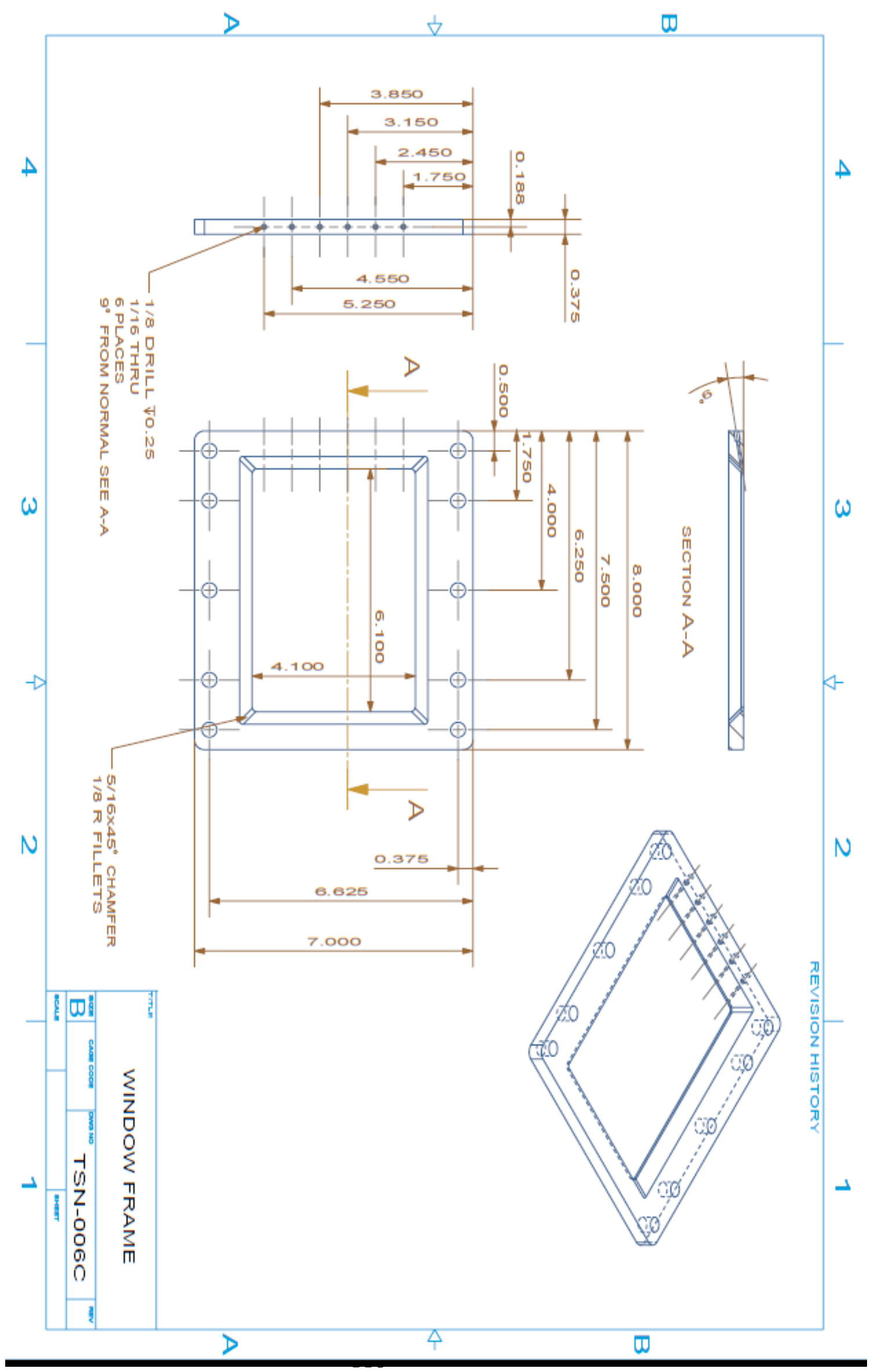




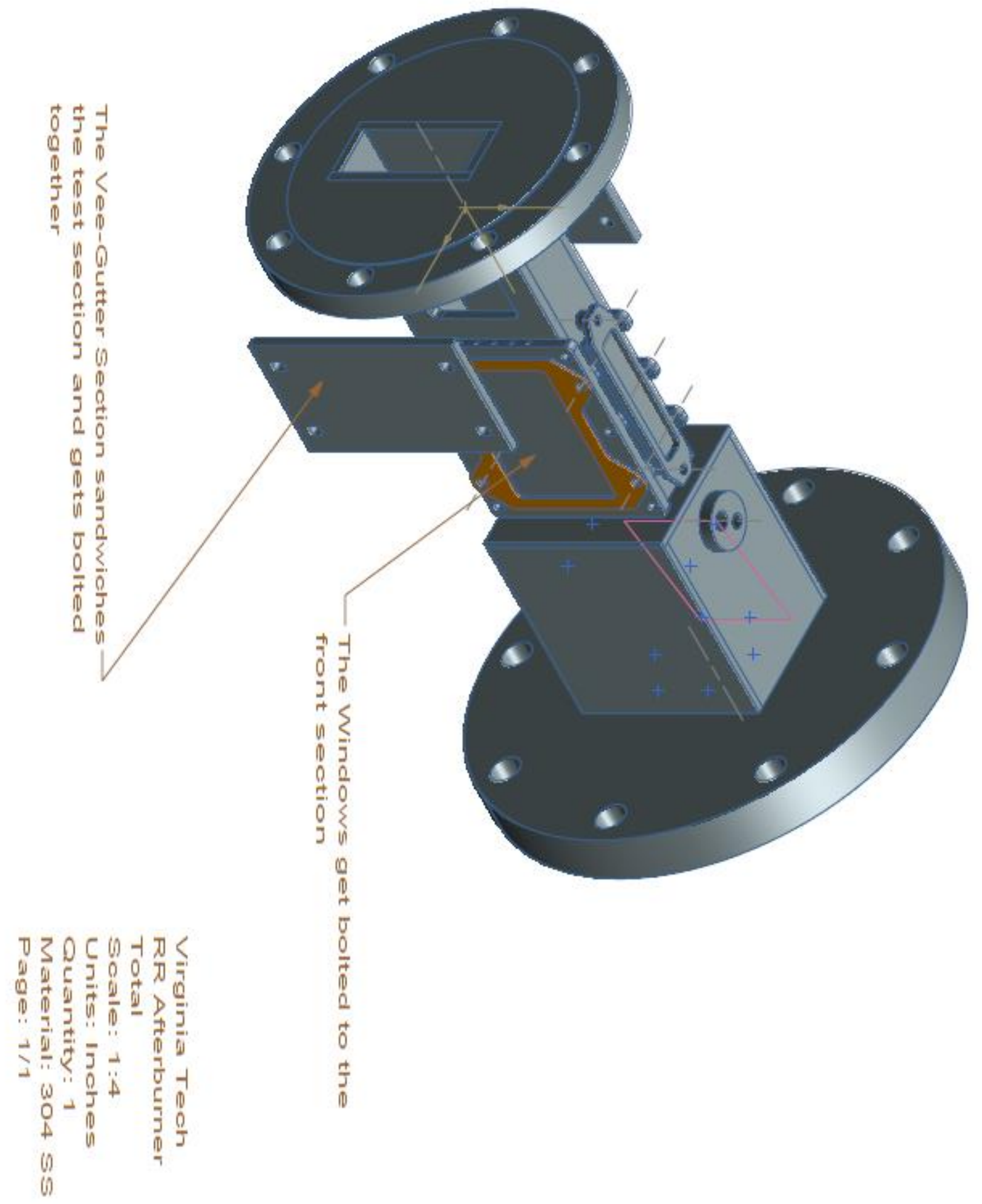



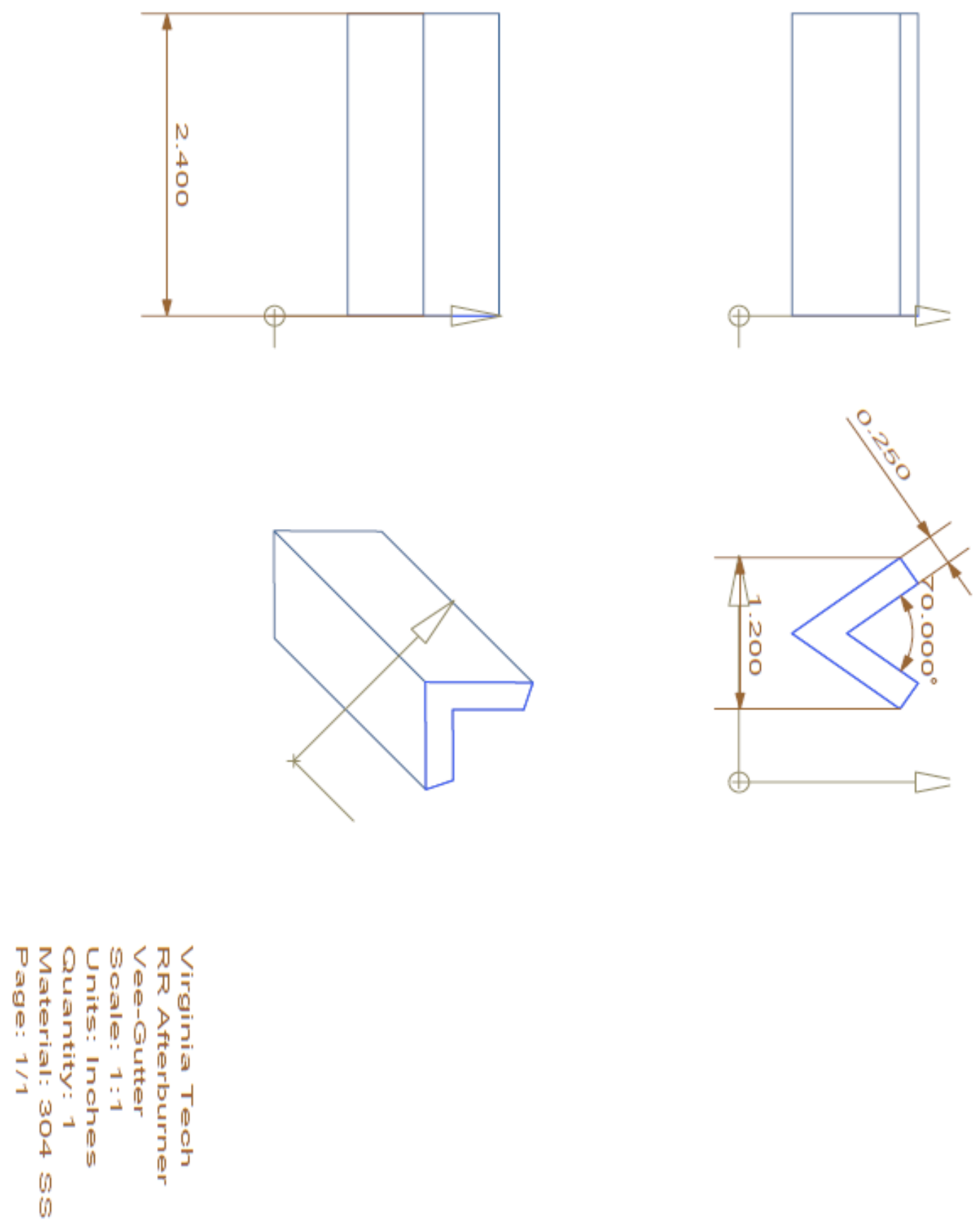


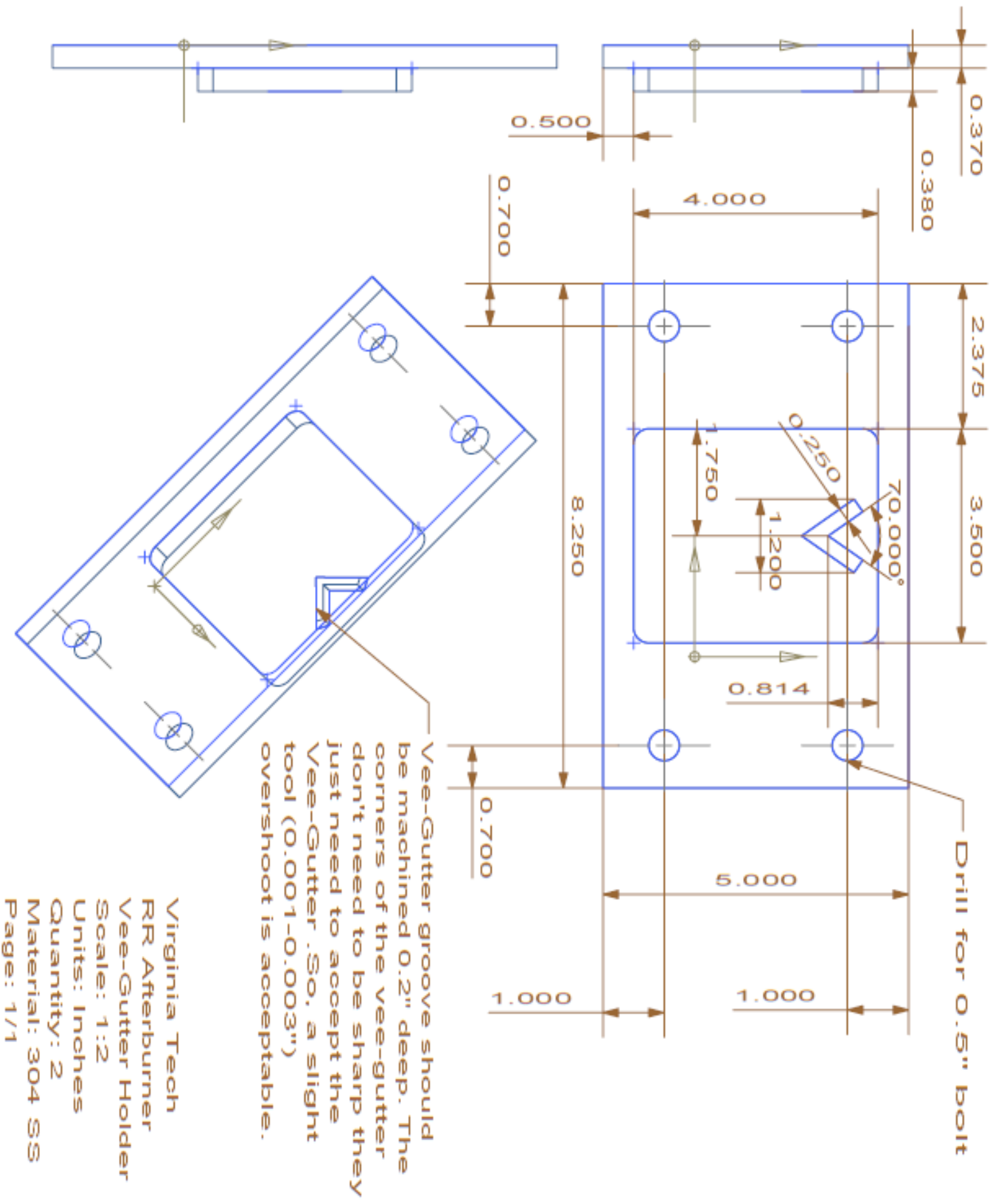




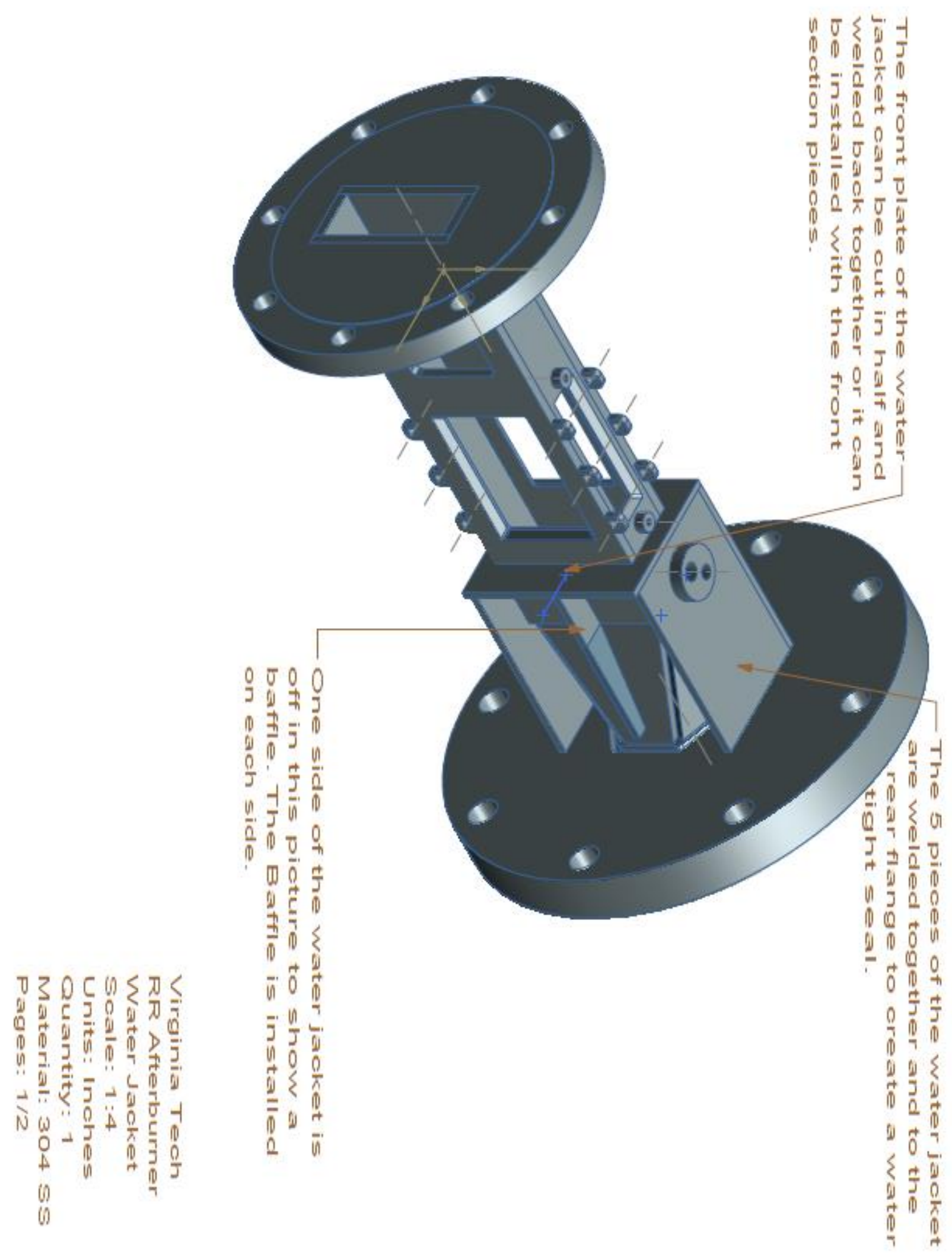



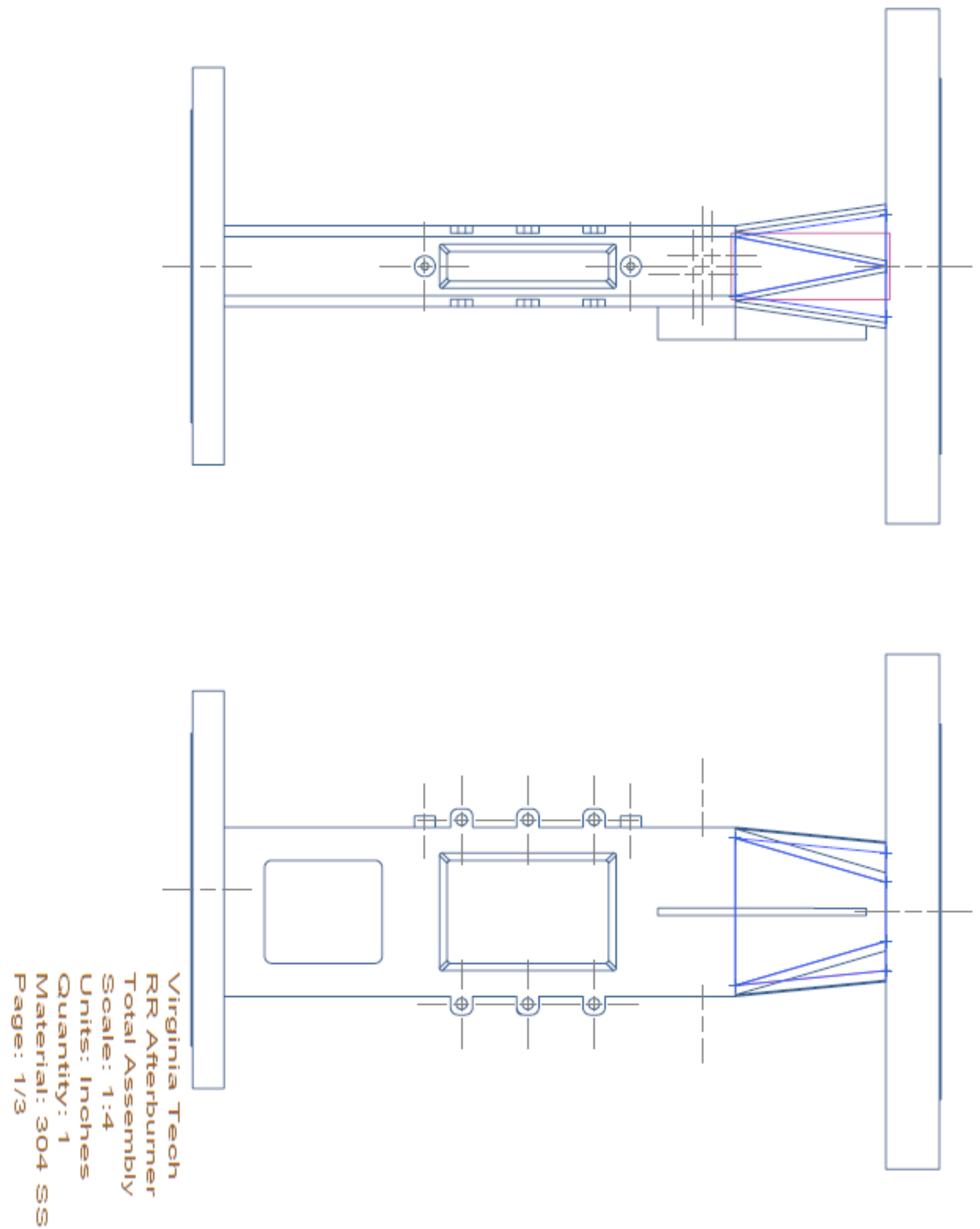


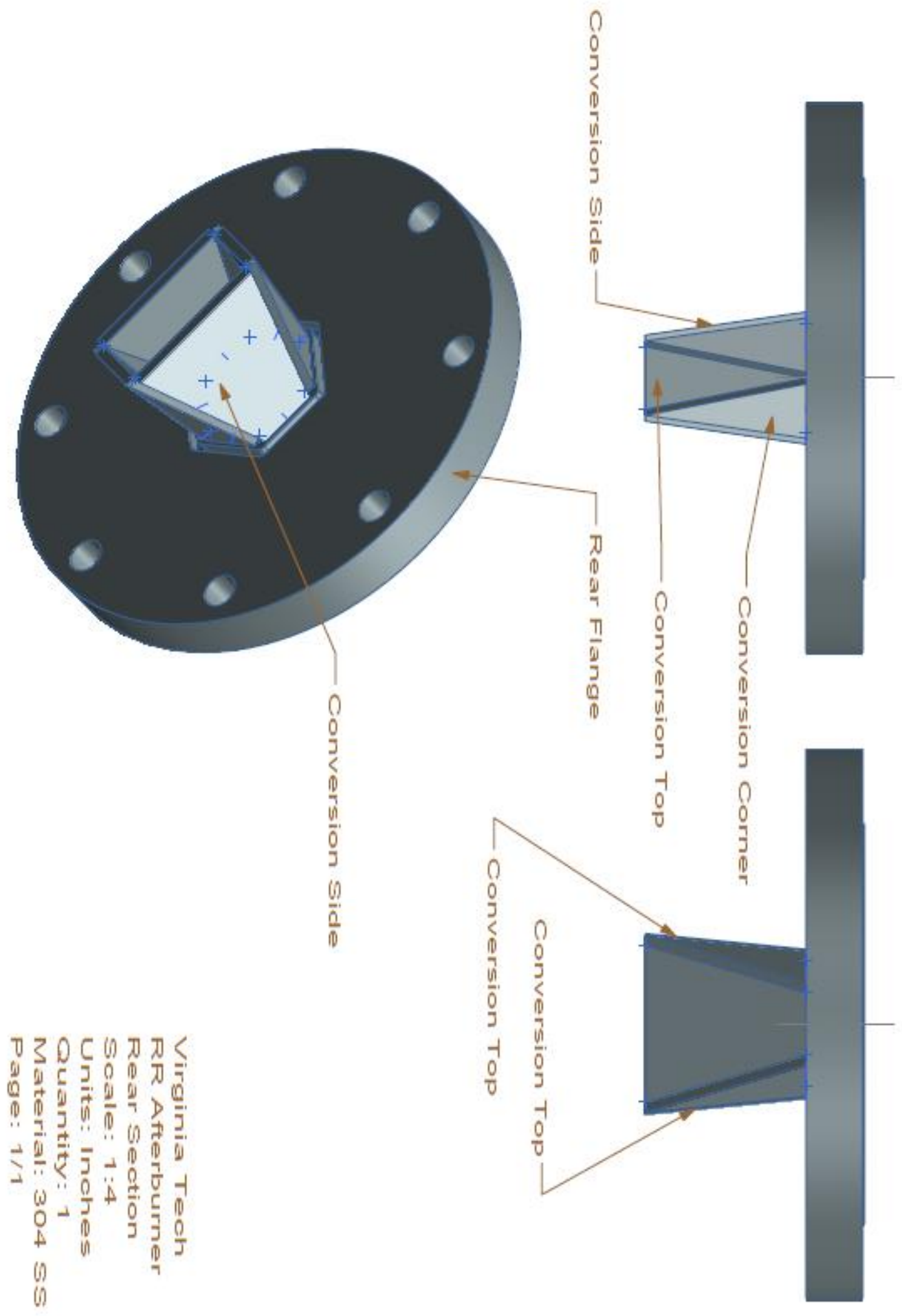




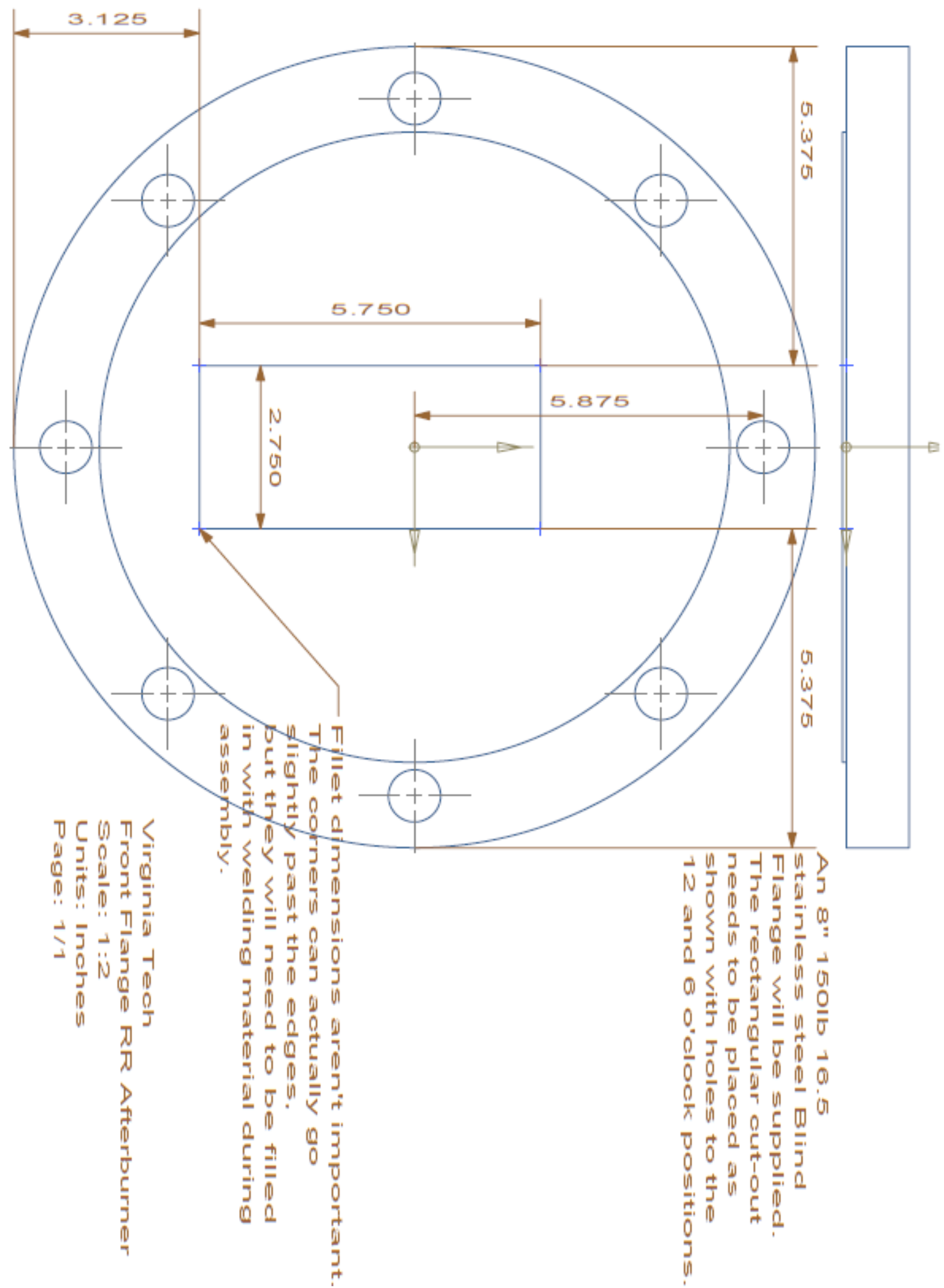




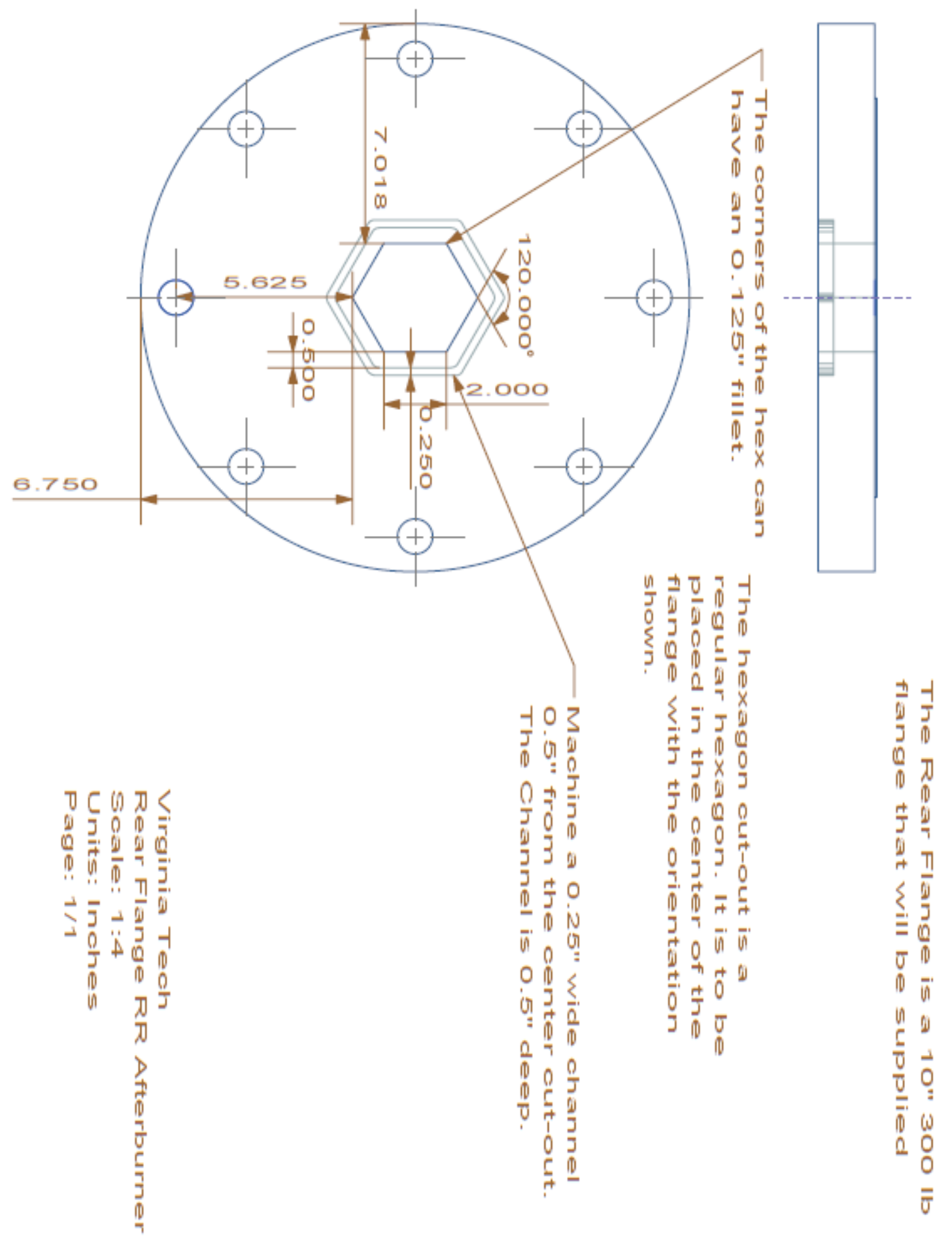




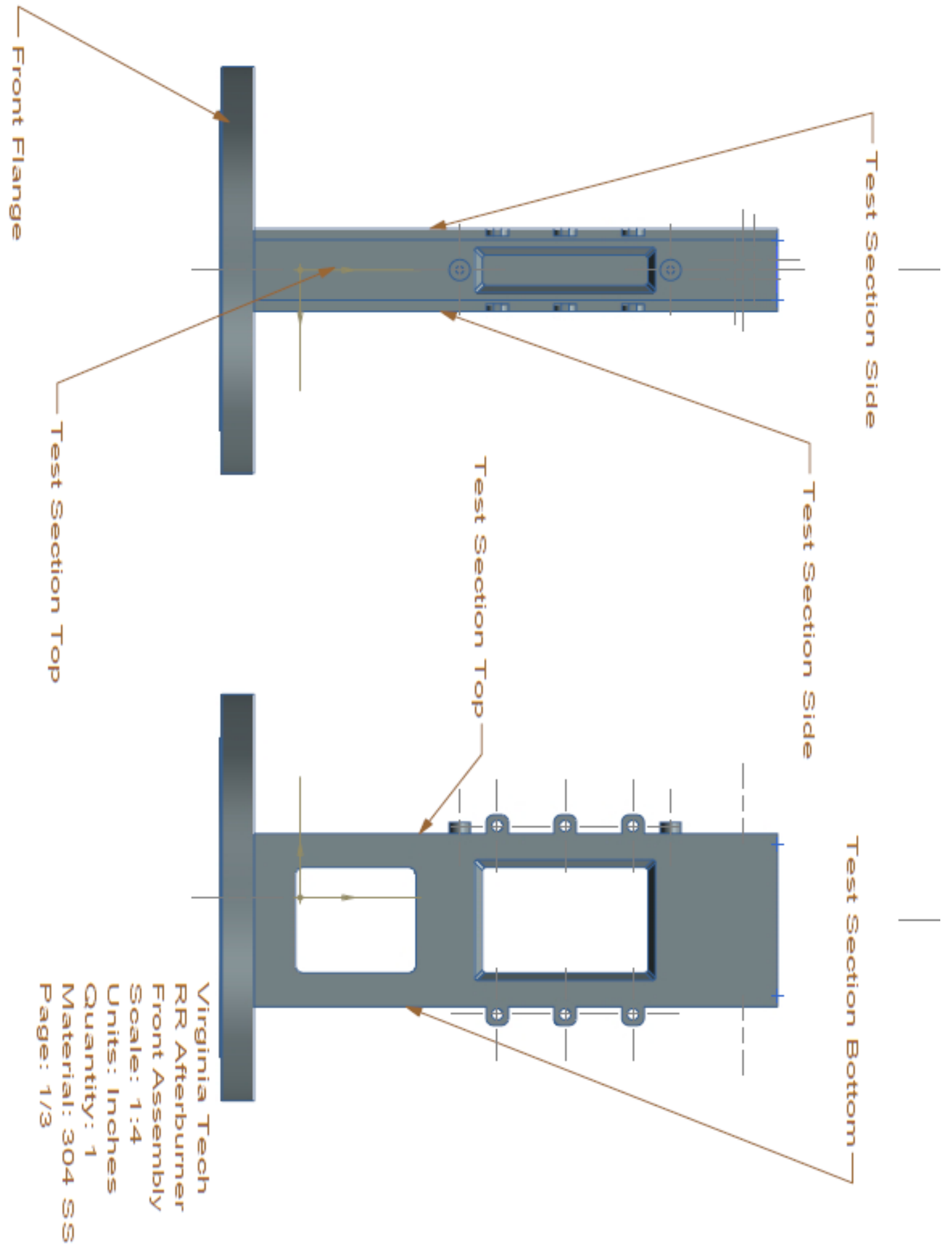




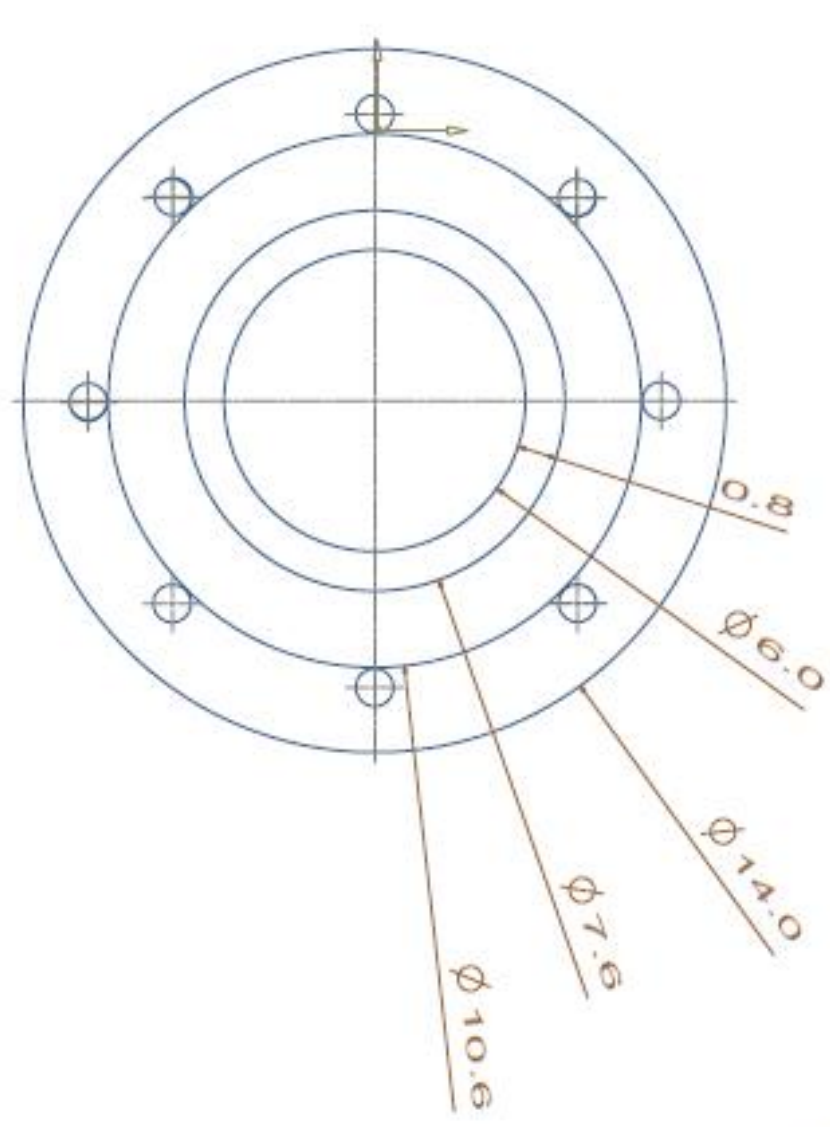

$\begin{array}{ll}j_{0} & 5 \\ 0 & 0 \\ 0 & \frac{1}{2} \\ 0 & 5 \\ 0 & 0\end{array}$

उ)

$\int_{0}^{3} \frac{1}{0} \frac{1}{0}$

$\frac{0}{0}$ (1)

$030 \subset$ प

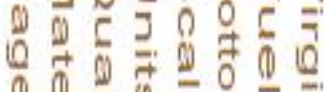

0 $\frac{0}{2}=0 \frac{0}{0}$

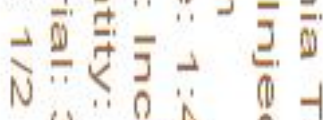

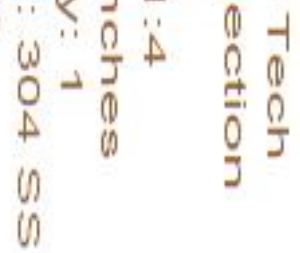

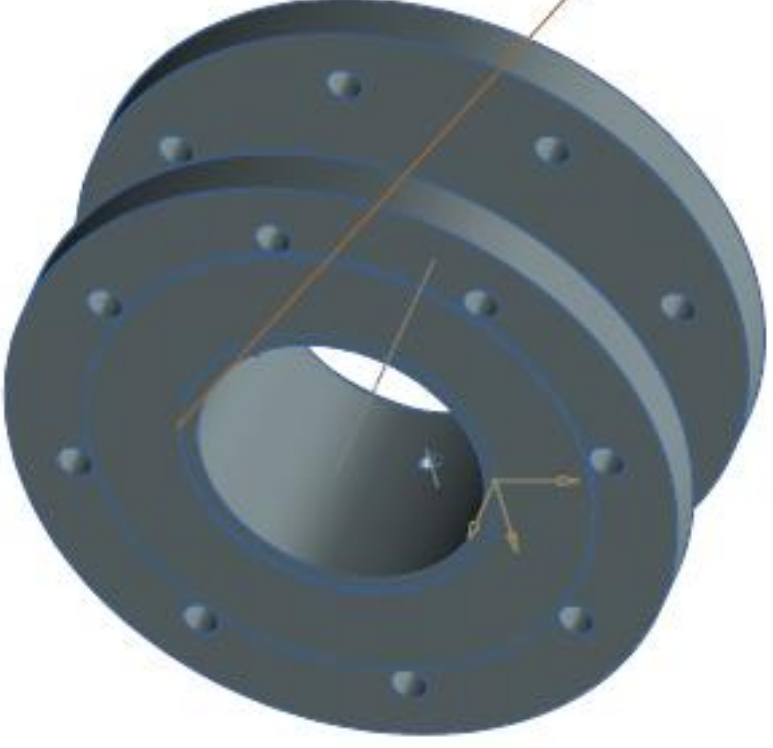



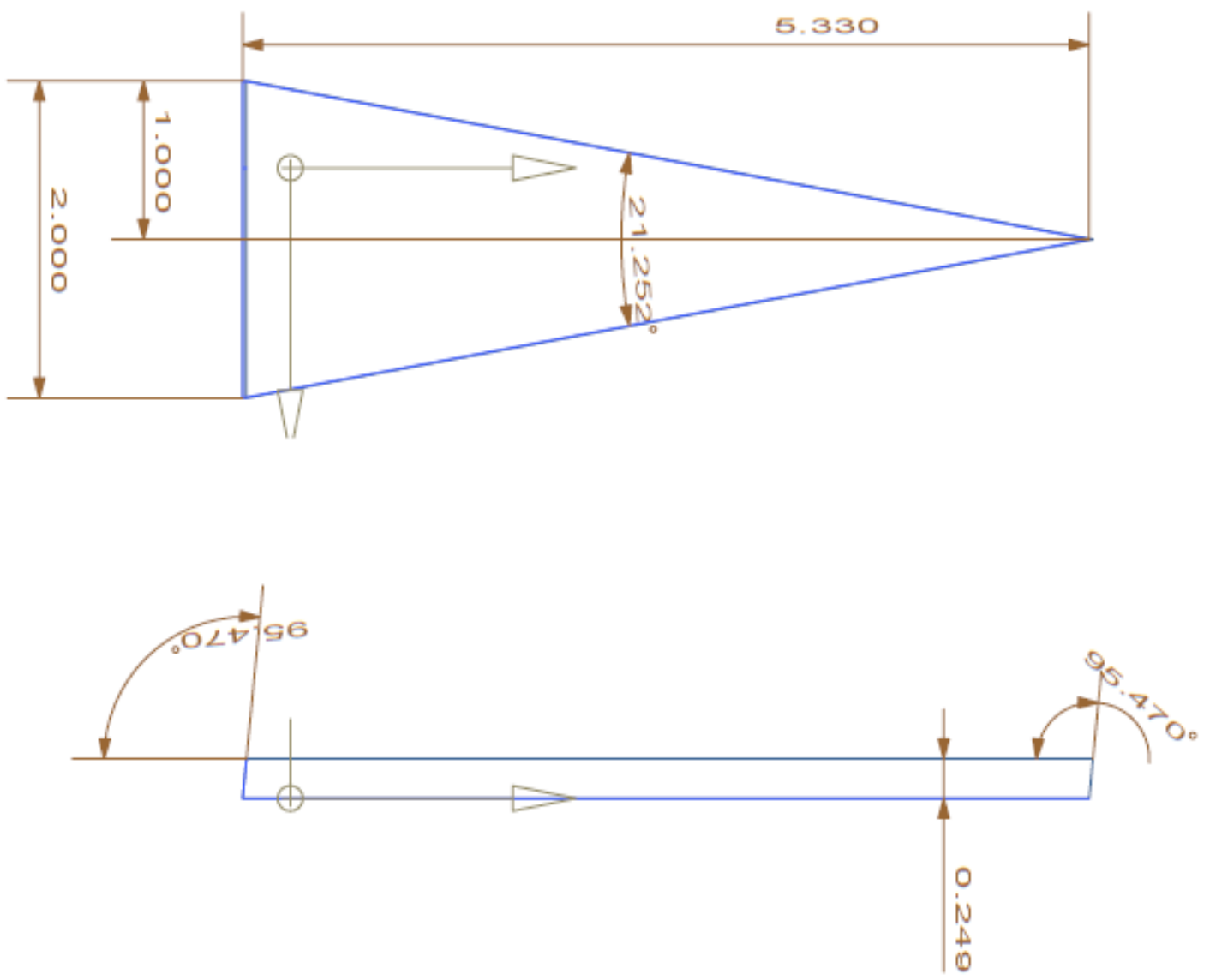

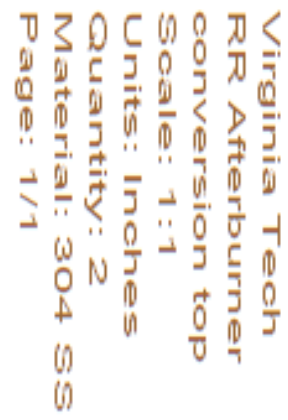




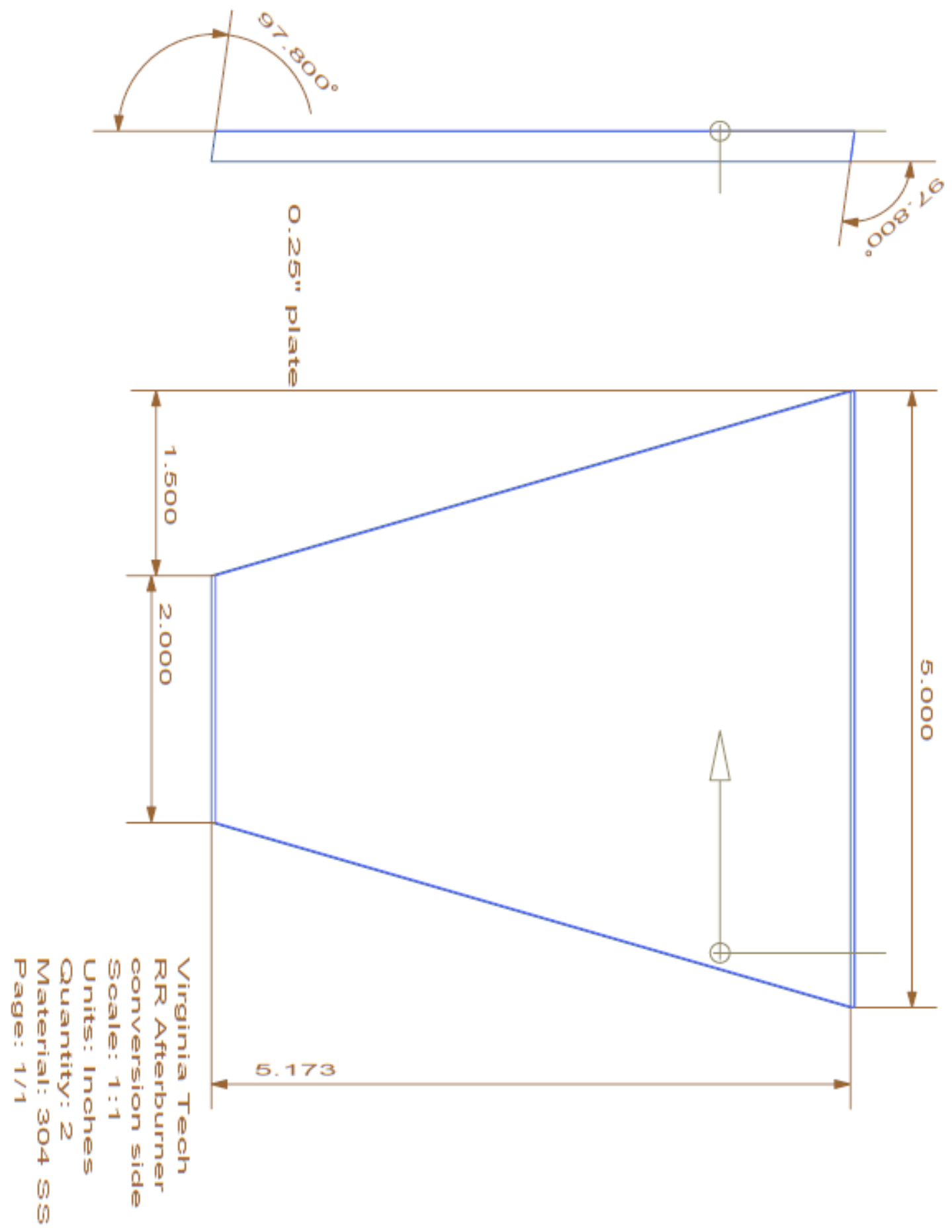




\section{B APPENDIX: Fluent UDF Codes}

\#include "udf.h"

\#include "sg_pdf.h"

DEFINE_SOURCE(chemil_src,c,t,ds,eqn)

\{

real source;

source=C_PREMIXC_RATE $(\mathrm{c}, \mathrm{t})$;

C_UDMI $(\mathrm{c}, \mathrm{t}, 0)=$ source;

return source;

\}

DEFINE_UDS_FLUX(chemil_flux,f,t,i)

\{

real sightvector[ND_ND],A[ND_ND],flux;

sightvector $[0]=0$;

sightvector $[1]=0$;

sightvector[2]=-1;

F_AREA(A,f,t);

flux=NV_DOT(sightvector,A);

return flux;

\} 


\section{APPENDIX: Data Reduction Codes}

\section{C.1 Vector POD Code}

function [Umodesgrid, UmodesVec, eVecs, Ubargrid, Urmsgrid, modeweights, modeweightsnorm, rawgrid]=vector_POD(Uall,dimensions, Nx, Ny, snapshots)

o POD Function for scalar field values on regular grid ( $\mathrm{Nx} x$ Ny = samples)

Uall should be of size (samples*dimensions, snapshots)

\% Code Performs mean subtraction of original data

\% Code weights each by its energy

\% Returns Umodesgrid which is a 4-D array indexed as follows

\% Umodesgrid (row, column, mode, dimension)

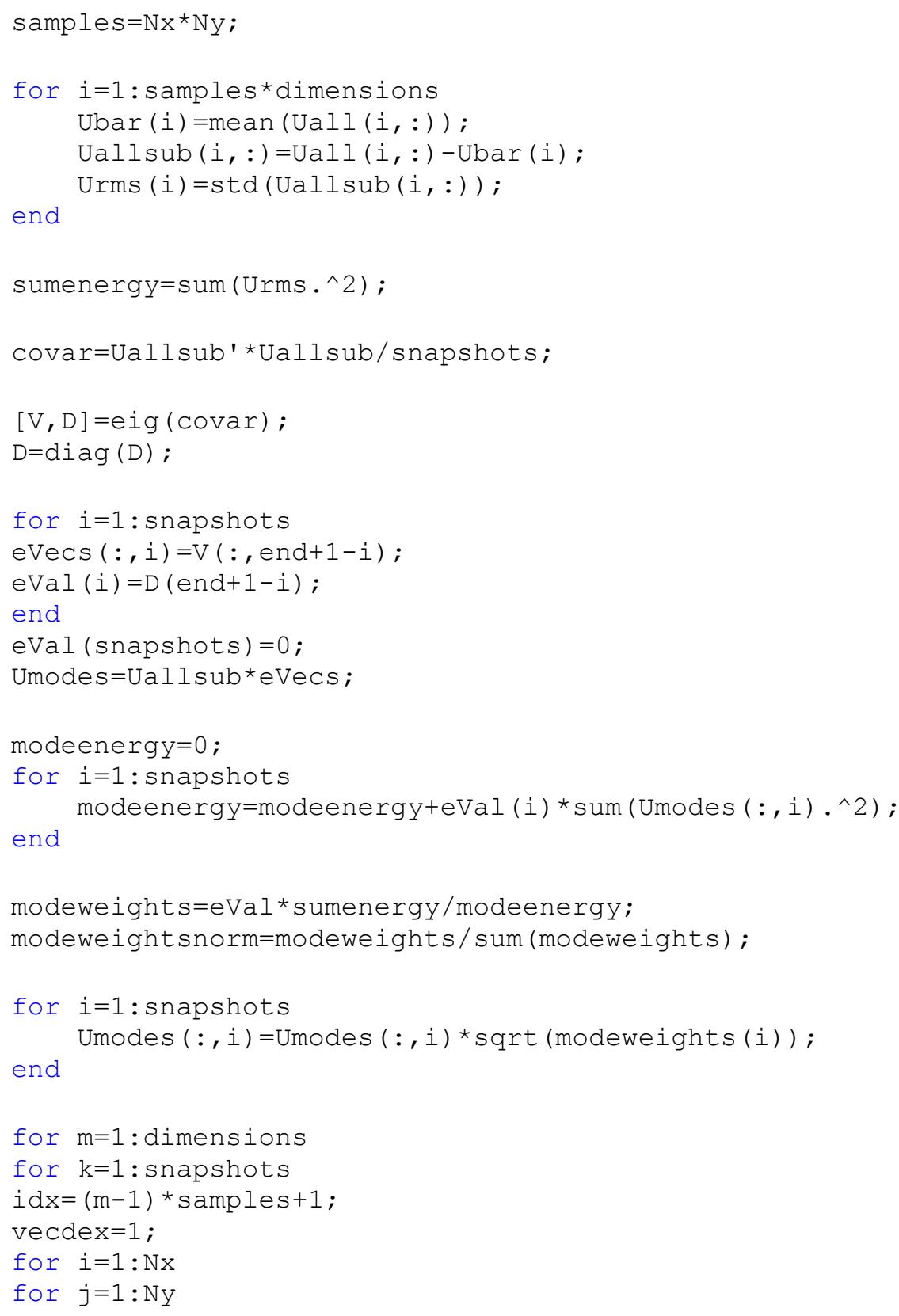




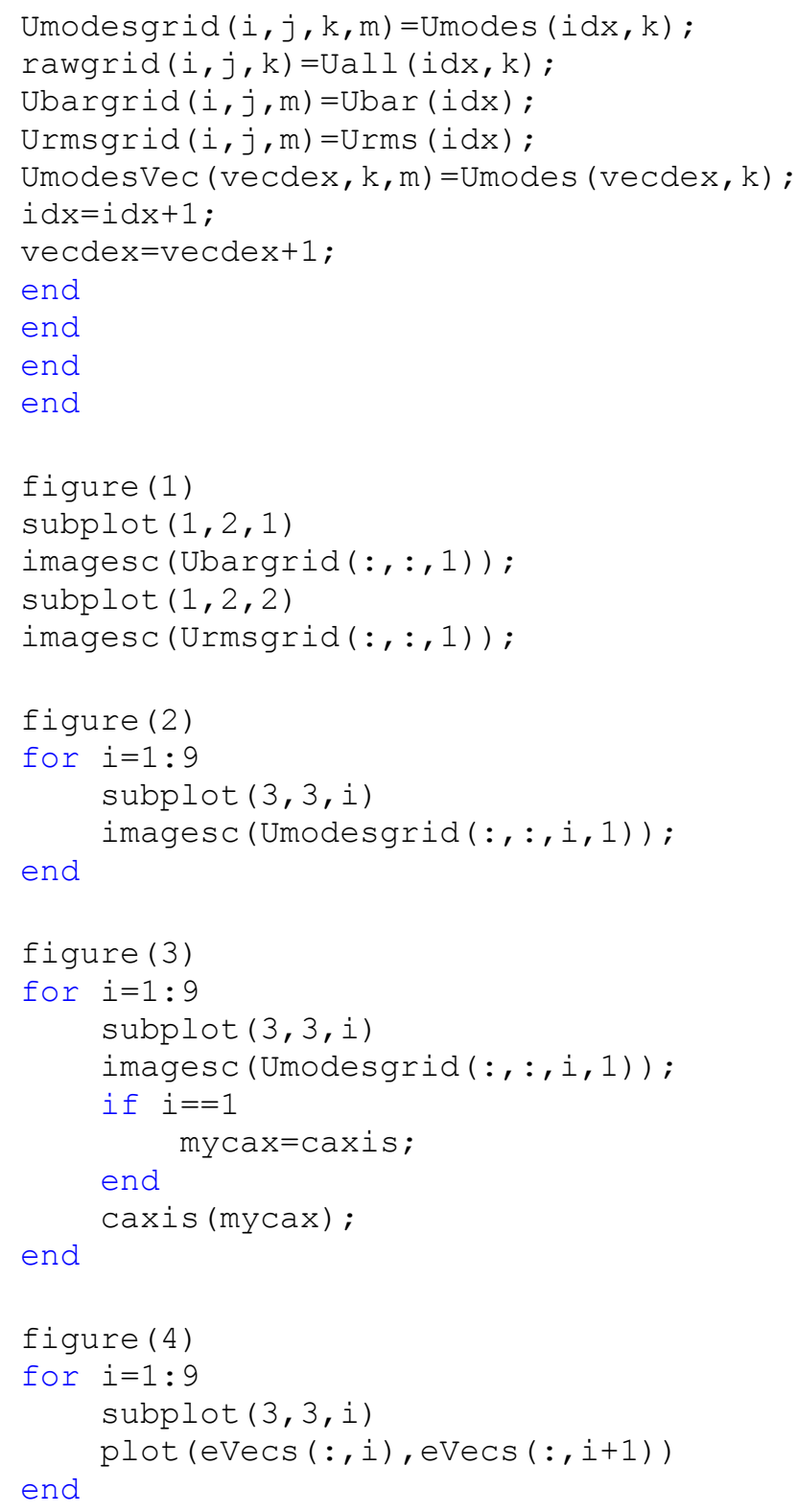




\section{C.2 FFT Code}

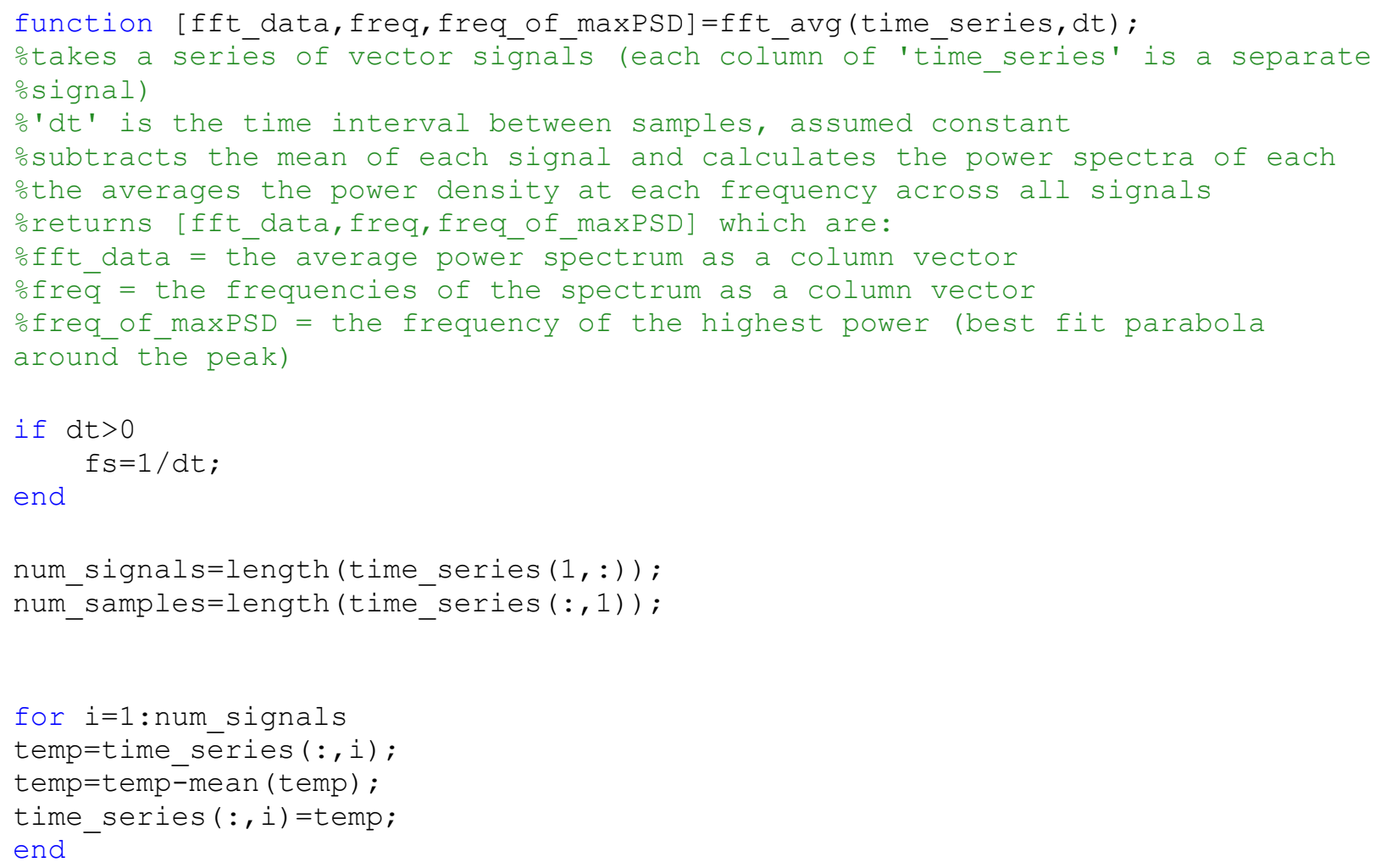




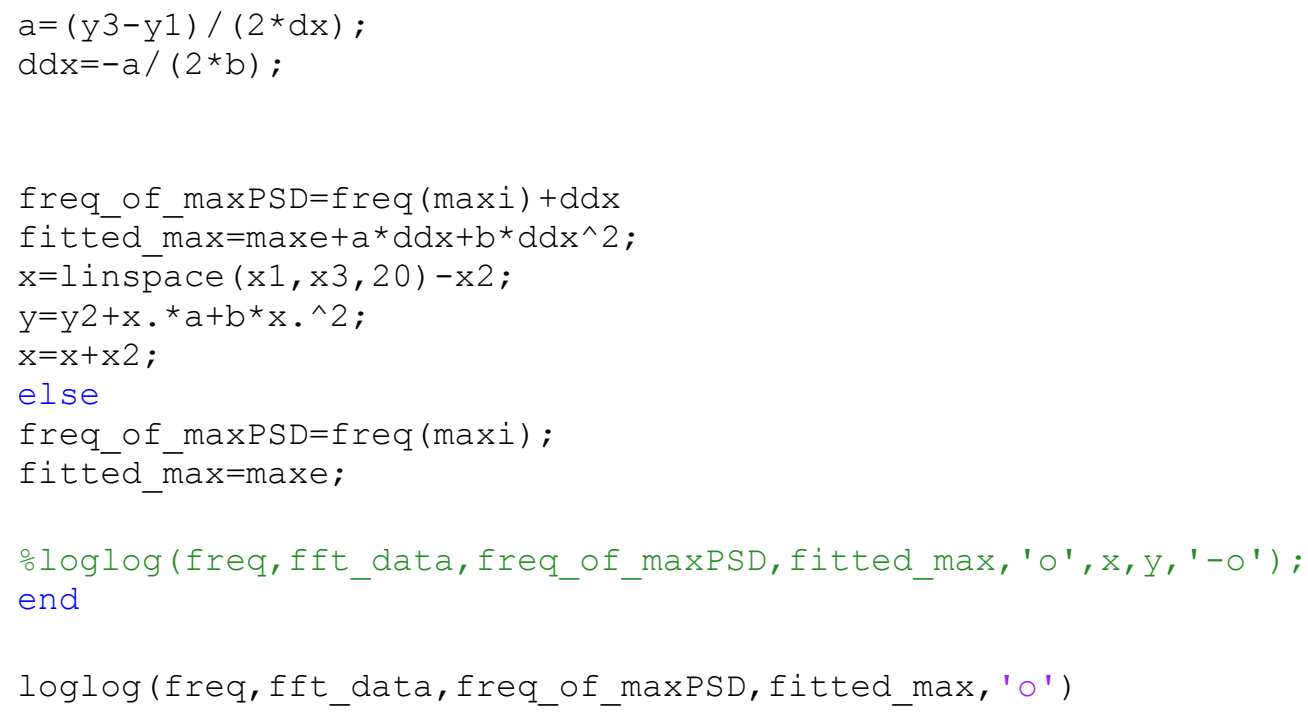

\section{C.3 Mode Aligning Code}

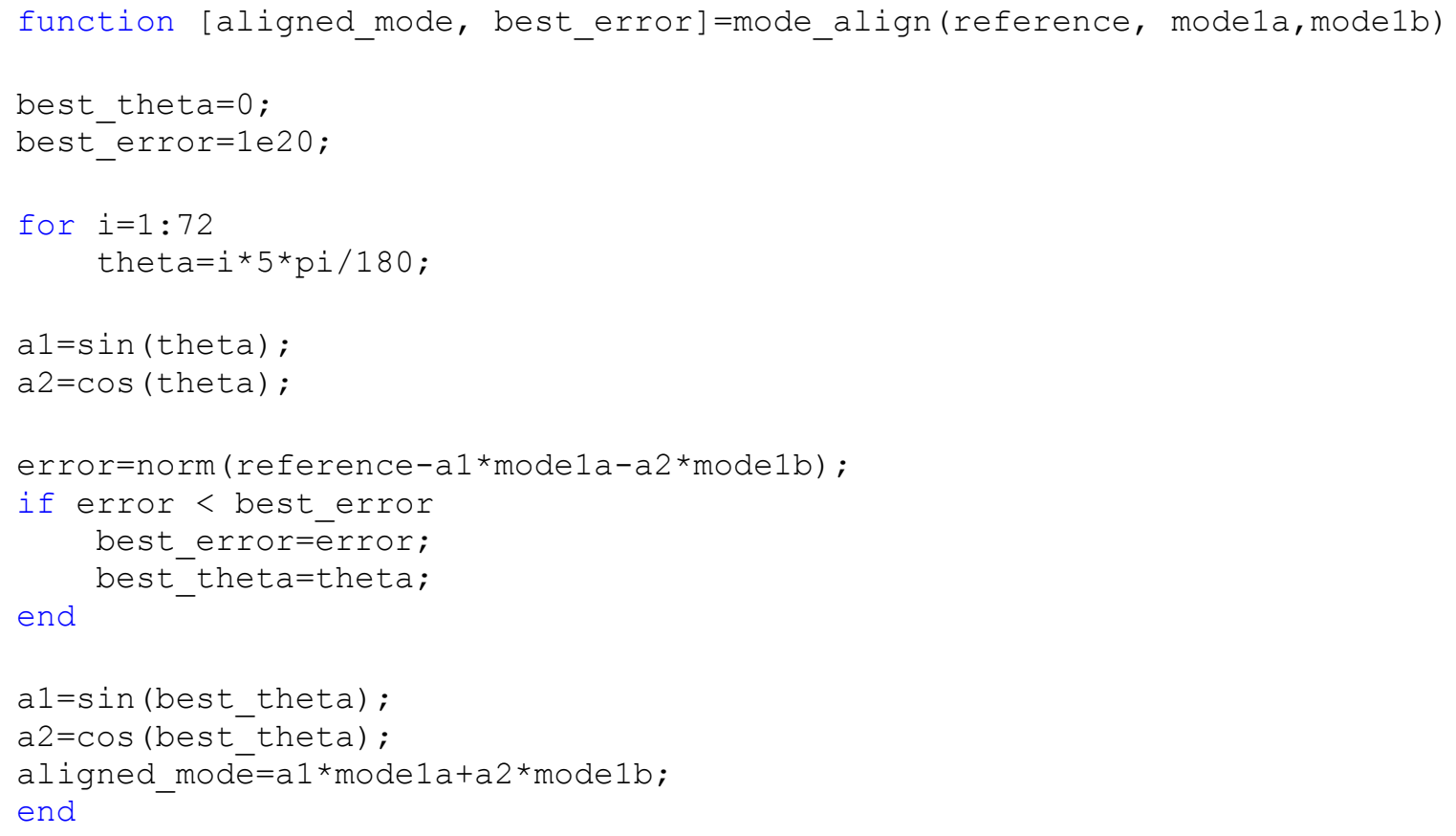

\section{C.4 Scalar Mode Plotter With Vee Gutter}

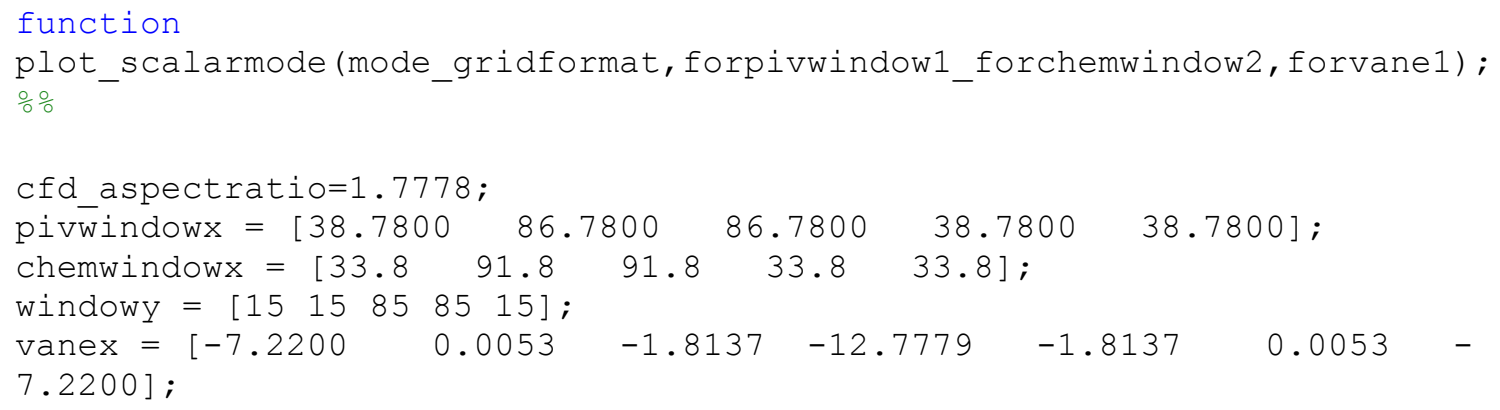




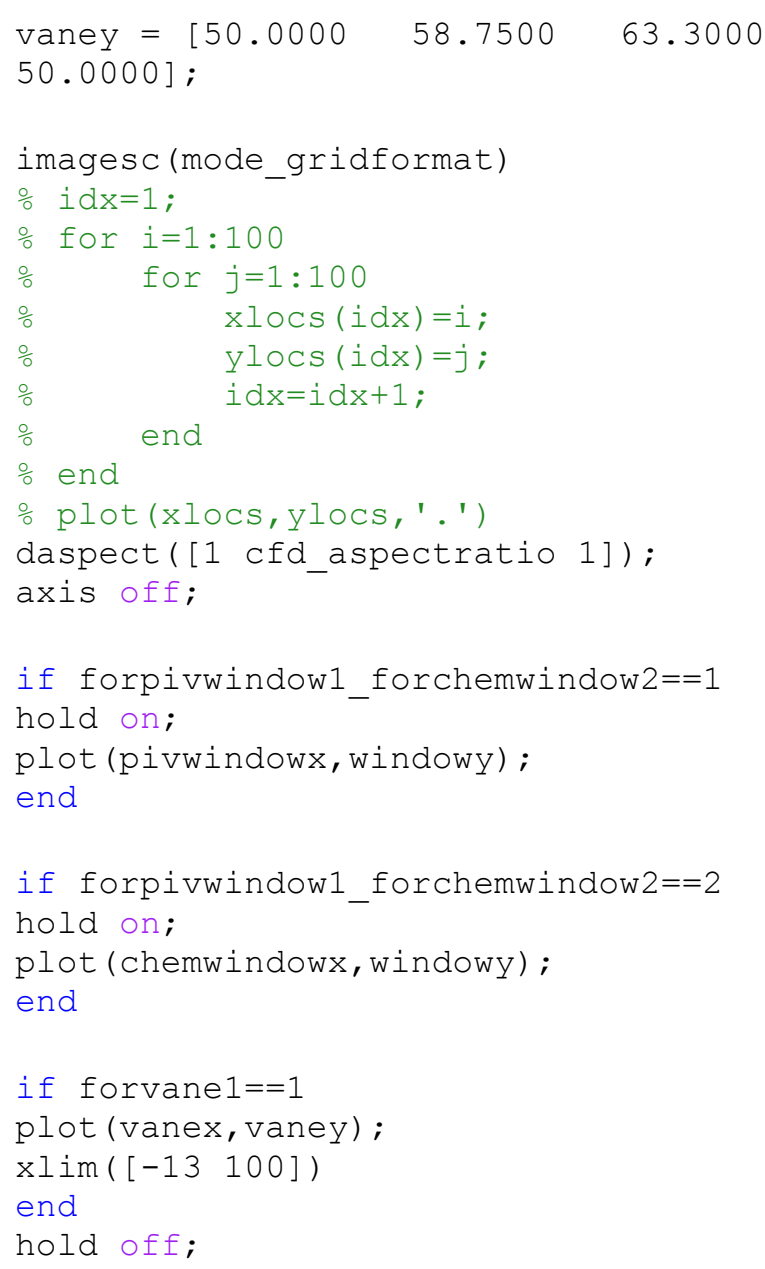

